\title{
Parameter Estimation Techniques for Ultrasound Phase Reconstruction
}

By

\section{Fatemeh Vakhshiteh}

\author{
A thesis submitted to \\ The Faculty of Graduate Studies and Research \\ in partial fulfilment of the degree requirements of
}

\section{Master of Applied Science}

Ottawa-Carleton Institute of Electrical and Computer Engineering

\author{
Department of Systems and Computer Engineering \\ Carleton University \\ Ottawa, Ontario, Canada
}

September 2010

Copyright $\odot$ Fatemeh Vakhshiteh, 2010 
Library and Archives Canada

Published Heritage Branch

395 Wellington Street Ottawa ON K1A ON4 Canada
Bibliothèque et

Archives Canada

Direction du

Patrimoine de l'édition

395, rue Wellington

Ottawa ON K1A ON4

Canada
Your file Votre référence
ISBN: 978-0-494-71557-4
Our file Notre référence
ISBN: $978-0-494-71557-4$
NOTICE:

The author has granted a nonexclusive license allowing Library and Archives Canada to reproduce, publish, archive, preserve, conserve, communicate to the public by telecommunication or on the Internet, loan, distribute and sell theses worldwide, for commercial or noncommercial purposes, in microform, paper, electronic and/or any other formats.

The author retains copyright ownership and moral rights in this thesis. Neither the thesis nor substantial extracts from it may be printed or otherwise reproduced without the author's permission.
AVIS:

L'auteur a accordé une licence non exclusive permettant à la Bibliothèque et Archives Canada de reproduire, publier, archiver, sauvegarder, conserver, transmettre au public par télécommunication ou par l'Internet, prêter, distribuer et vendre des thèses partout dans le monde, à des fins commerciales ou autres, sur support microforme, papier, électronique et/ou autres formats.

L'auteur conserve la propriété du droit d'auteur et des droits moraux qui protège cette thèse. $\mathrm{Ni}$ la thèse ni des extraits substantiels de celle-ci ne doivent être imprimés ou autrement reproduits sans son autorisation.
In compliance with the Canadian Privacy Act some supporting forms may have been removed from this thesis.

While these forms may be included in the document page count, their removal does not represent any loss of content from the thesis.
Conformément à la loi canadienne sur la protection de la vie privée, quelques formulaires secondaires ont été enlevés de cette thèse.

Bien que ces formulaires aient inclus dans la pagination, il n'y aura aucun contenu manquant. 
The undersigned recommend to the Faculty of Graduate Studies and Research acceptance of the thesis

\title{
Parameter Estimation Techniques for Ultrasound Phase Reconstruction
}

\author{
Submitted by Fatemeh Vakhshiteh, B.A.Sc. \\ in partial fulfilment of the requirements for the degree of \\ Master of Applied Science
}

A. Adler, Thesis Supervisor

Y. Ono, Thesis Co-Supervisor

H. Schwartz

Chair, Department of Systems and Computer Engineering

Carleton University 


\begin{abstract}
Elastography technique can measure stiffness which is a sign commonly noticed in muscular disorders or athletic injuries. Focusing on ultrasound compression elastography technique, this work aims to measure phase shift between ultrasound received RF signals which in turn can be used for stiffness (strain) estimation. To measure the phase information, quadrature phase detection technique was employed. Then, in an investigation, certain parameters that affect its functionality and outcomes (e.g., signal to noise ratio (SNR) and bandwidth) were explored. It was observed that by the SNR equal to $60 \mathrm{~dB}$, the phase shift error is around 0.2 rad (equivalent to displacement error of $4.9 \mu m \iota)$ while it increases with decreases in SNR until becomes 18.03 $\operatorname{rad}($ equal to displacement error of $442.09 \mu \mathrm{m}$ ) with the SNR of $0 \mathrm{~dB}$. To reconstruct the less accurate phase outcomes of the quadrature method, a new algorithm was designed based on Gauss-Newton method. Using appropriate regularization parameter defined in this algorithm resulted in the reconstructed phase shift with the error around 14 rad (corresponding to displacement error of $343.28 \mu \mathrm{m}$ ) when the SNR was $0 \mathrm{~dB}$. It was concluded that, regularization parameter can act as filter coefficients with the ability to remove noise appeared in the phase measurements.
\end{abstract}




\section{Acknowledgements}

It would be impossible for me to thank the merciful God who has supported me with his generosity and kindness, helped me to go forward and not to be disappointed, inspired me to think properly, and never left me alone, even for a second. I appreciate my God from the depths of my heart.

I would like to thank my supervisor, Dr. Andy Adler, Professor of Systems and Computer Engineering, Carleton University for his friendship guidance, inspiration and assistance and my co-supervisor, Dr. Yuu Ono, Associate Professor of Systems and Computer Engineering, Carleton University for his expertise and guidance. I appreciate both the time and the effort that they provided me with throughout my graduate studies.

I am grateful to my colleagues Yasheng Maimaitijiang, Jason 1. Silver, and Vilas Joshi for their friendship, efforts and assistance throughout my research work.

A depth of gratitude are due my family: Javad Vakhshiteh, my father, who has supported me emotionally and guided me deeply to solve my problems; Ehteram Mahoodi, my mother, who has provided me with her enormous kindness, love and compassionate; Faezeh Vakhshiteh, my older sister, who has inspired confidence in me and provided me with her advice.

Special thanks to Farzaneh Vakhshiteh, my younger sister, who has stayed days and nights with me and supported me with her huge considerations and helps.

I would also like to express my appreciation to Carleton University and the Natural Sciences and Engineering Research Council of Canada (NSERC) for the opportunity and the financial support that they provided for this research. 


\section{Table of Contents}

$\begin{array}{ll}\text { Abstract } & \text { i }\end{array}$

Acknowledgements

Table of Contents

List of Figures $\quad$ vi

Chapter 1: Introduction ............................................................................................................. 1

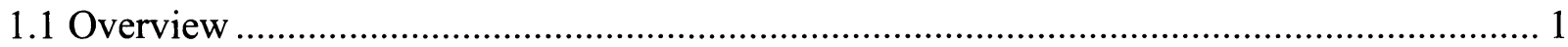

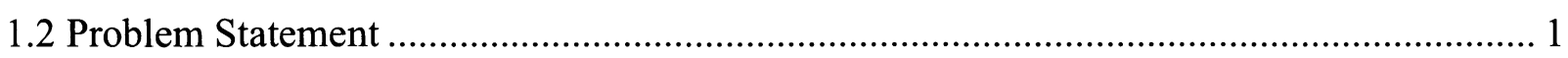

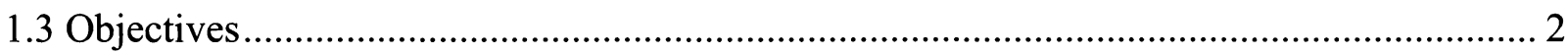

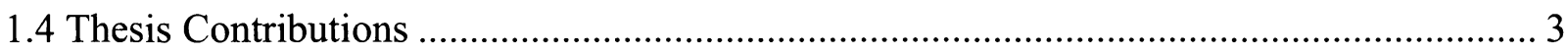

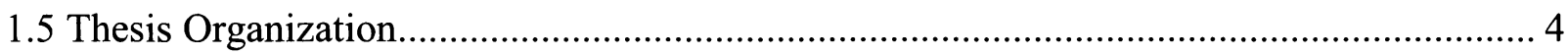

Chapter 2: Background Review .......................................................................................................... 5

2.1 Human Muscular System ................................................................................... 5

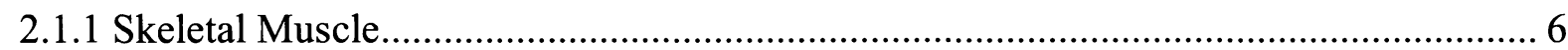

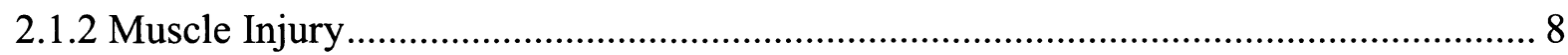

2.1.3 Diagnosis and Monitoring by Ultrasound .......................................................... 12

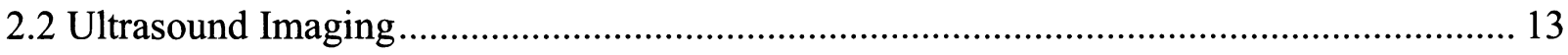

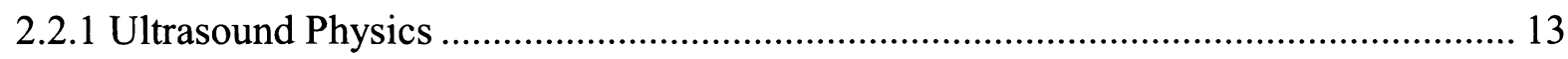

2.2.2 Ultrasonic Transducer Elements and Probes ..................................................... 18

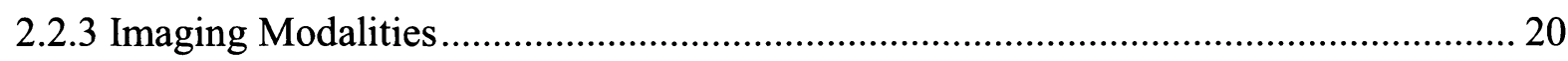

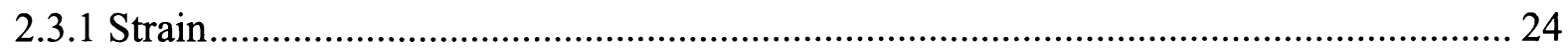

2.3.2 Ultrasound-based Elastography.................................................................... 25

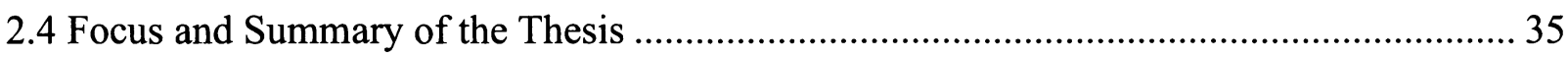

Chapter 3: Review of Data Acquisition Method ............................................................. 36

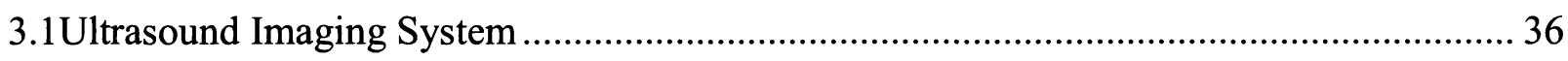

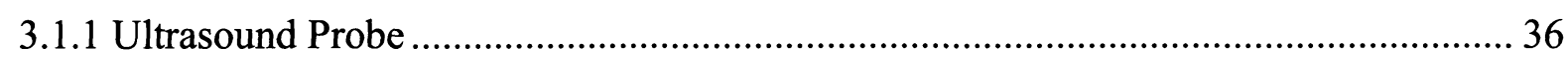

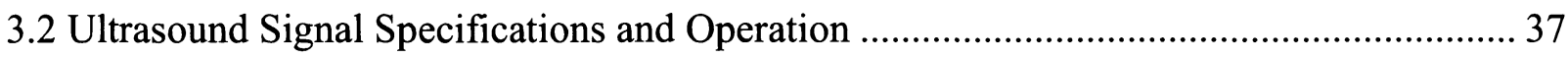




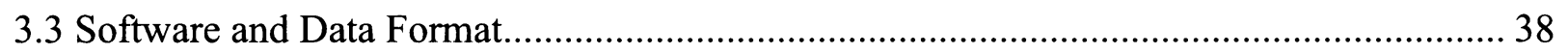

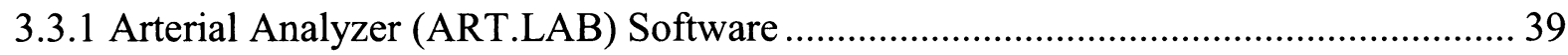

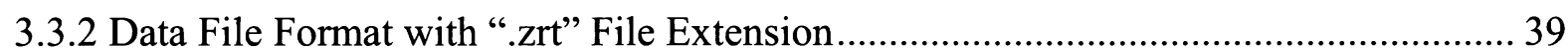

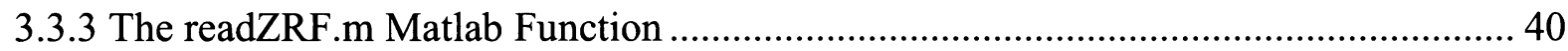

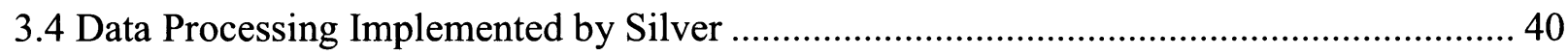

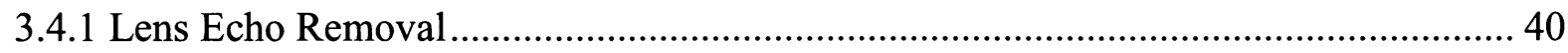

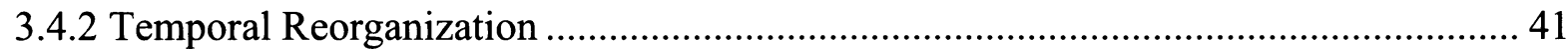

3.5 Data Files (“.zrf” files) and Experimental Testing............................................................. 42

Chapter 4: Simulation of Ultrasound Speckle echoes ........................................................................... 44

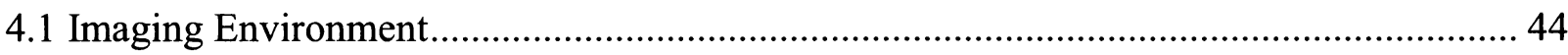

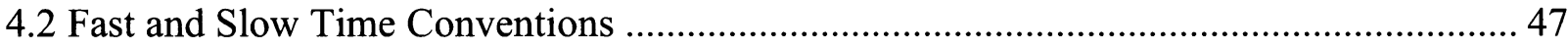

4.3 Scatterers Contribution to Ultrasound Received RF Signal................................................ 48

4.4 Scatterers Contribution in a Deformed Medium ............................................................... 50

4.5 Computer Simulation .................................................................................................. 51

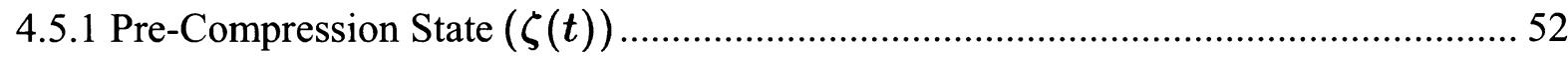

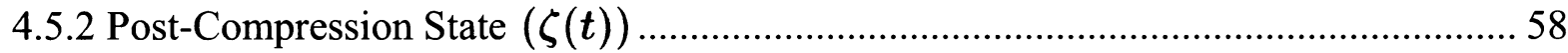

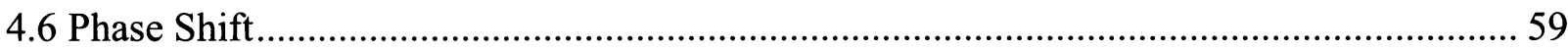

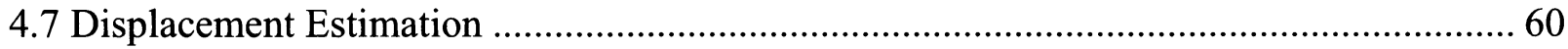

4.7.1 Autocorrelation-based Quadrature Detection Technique ............................................. 61

4.7.2 Phase Subtraction-based Quadrature Detection Technique......................................... 62

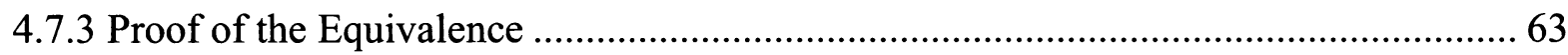

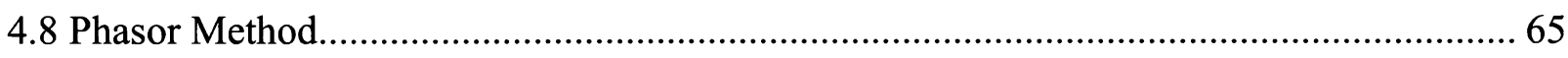

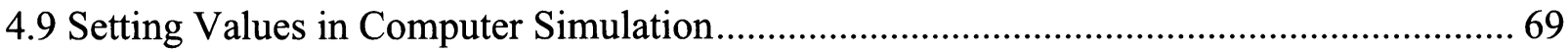

Chapter 5: Numerical Simulation Results ........................................................................................ 73

5.1 Instantaneous Displacement Effect on Consecutive Phase Shift ....................................... 73

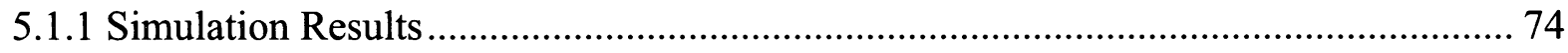

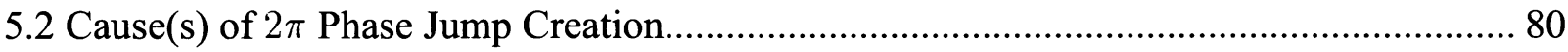

5.2.1 $\Delta d_{z}(N, m)$ Boundary to Avoid Phase Jumps............................................................ 82

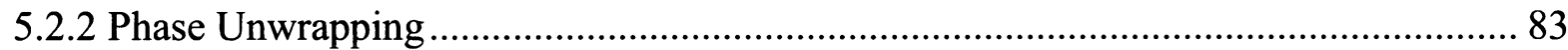

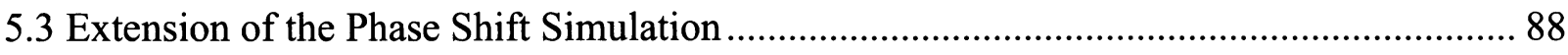

5.3.1 Accumulated Phase Shift Equivalent to $1 \mathrm{~mm}$ Displacement of the Volume ............ 90 
5.4 Quadrature Technique Performance in Different Conditions

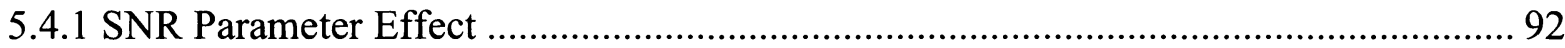

5.4.2 Number of Scatterers $(\boldsymbol{L})$ Parameter Effect ..................................................... 95

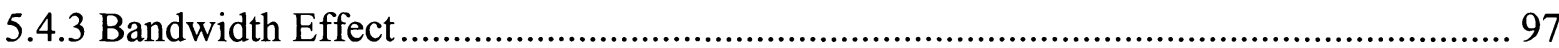

Chapter 6: Inverse Problem Techniques ........................................................................ 101

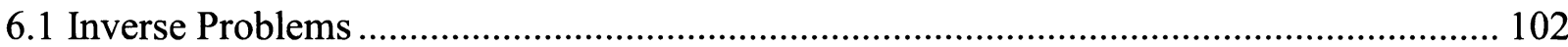

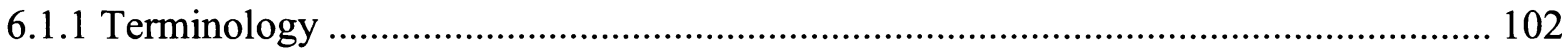

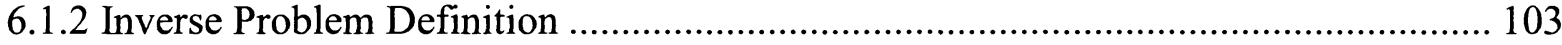

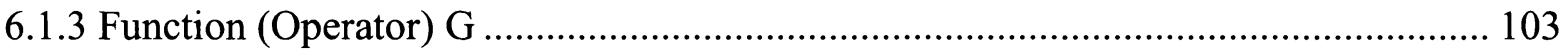

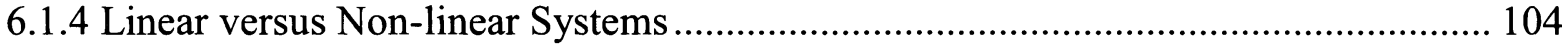

6.1.5 Overdetermined and Underdetermined Systems of Equations .............................. 104

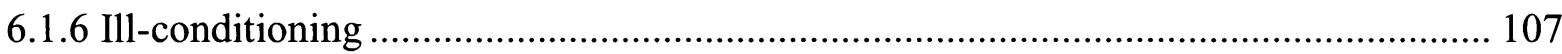

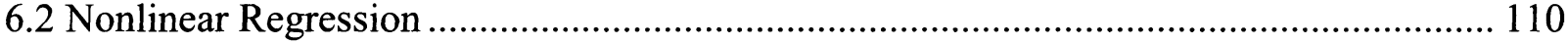

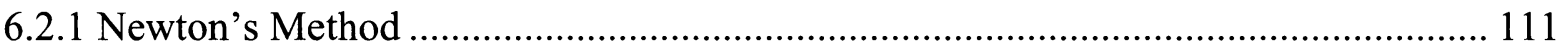

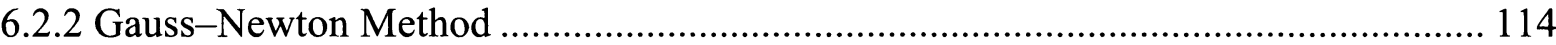

6.3 Defined Inverse Problem for this Research.......................................................... 118

6.3.1 Inverse Problem Determination .......................................................................... 118

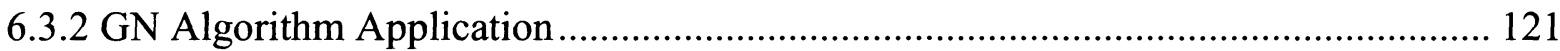

6.3.3 Reconstructed Phase (Phase Shift) Results via the GN Algorithm ......................... 123

6.3.4 Regularization Parameter Effect................................................................. 132

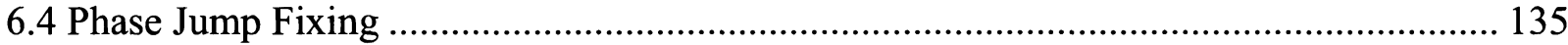

6.5 Comparison with the Linear Lowpass Filter ........................................................ 136

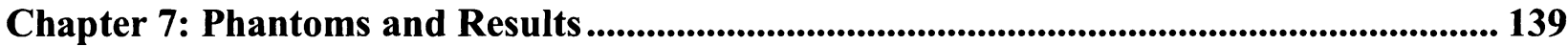

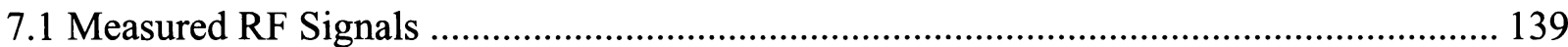

7.2 Phase Reconstruction Process ........................................................................... 140

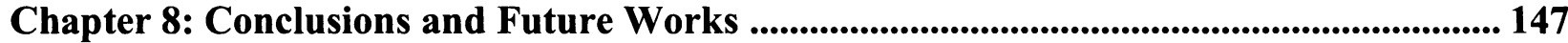

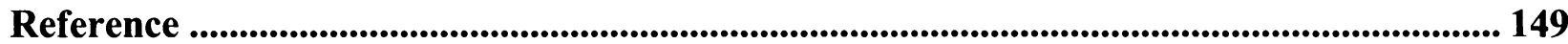




\section{List of Figures}

Figure 2. 1 Reflection caused by a sound wave striking a specular reflector at an angle.

Figure 2. 2 Reflection caused by a sound wave striking a specular reflector at normal incidenc 17

Figure 2. 3 Nonspecular reflections or scattering 18

Figure 2. 4 Construction of a single-element transducer 20

Figure 2. 5 An example of A-mode scan and its corresponding image ....................................... 21

Figure 2. 6 An example of B-mode scan and its subsequent image ......................................... 22

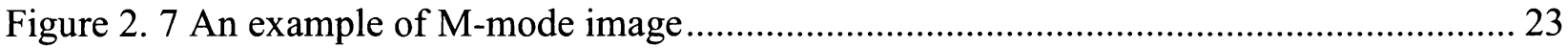

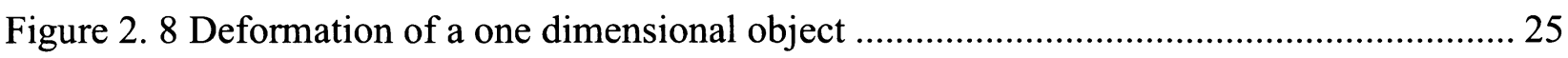

Figure 2. 9 Pre- and post-compression RF signals and the induced time shift representation ..... 26 Figure 2.10 Strain estimation by using local displacements between the first two consecutive windows 30

Figure 2.11 Curve of local displacement of the particles with respect to their position (depth) in a compressed normal tissue along with the corresponding strain curve.

Figure 2.12 Curve of local displacement of the particles with respect to their position (depth) in an injured muscle along with the corresponding strain curve. 32

Figure 3. 1 Picus ultrasound hardware. 37

Figure 3. 2 An example of measured RF signal stored in files with .zrf extensions 42

Figure 3. 3 An example of measured Z-signal stored in files with .zrf extensions ...................... 42

Figure 4. 1 A typical part of body which is being imaged .......................................................... 44

Figure 4. 2 Probe motion as observed with respect to the ultrasound probe reference frame...... 45

Figure 4. 3 Probe motion as observed with respect to the global reference frame........................ 46

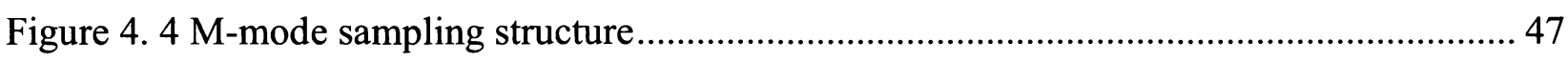

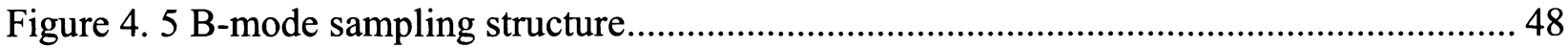

Figure 4. 6 A microscopic version of a fat part of body with a number of scatterers in it along

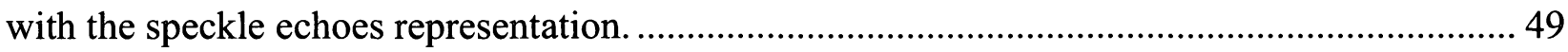

Figure 4. 7 Pre- and post-compression RF signals' representation ............................................. 51 
Figure 4. 8 A block diagram of the creation of an RF signal from the backscattered echoes ...... 55 Figure 4. 9 Summation of phasor parts of two complex sinusoids by graphical vector addition. 68 Figure 4. 10 Amplitude spectrum of a measured RF signal (real data) around the center frequency of $5 \mathrm{MHz}$ Figure 5. 1 Pre-compression RF signal along with its phase information that is measured by the

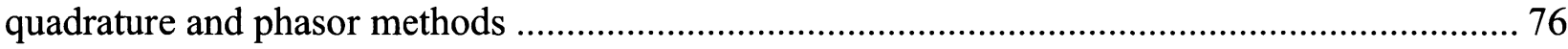

Figure 5. 2 Post-compression RF signal along with its phase information that is measured by the quadrature and phasor methods 76

Figure 5. 3 Phase shift between pre- and post-compression RF signals, equivalent to $50 \mu \mathrm{m}$ displacement of the sample volume after compression

Figure 5. 4 Phase shift between pre- and post-compression RF signals, equivalent to $0.1 \mu \mathrm{m}$ displacement of the sample volume after compression 78

Figure 5. 5 Accumulated phase shift resulted after 501 frames (RF signals) equivalent to $50 \mu \mathrm{m}$ displacement of the sample volume 79

Figure 5. $62 \pi$ phase jump creation as a result of movement from quadrant 3 to 2 (or vice versa)

Figure 5. 7 Phase shift between pre- and post-compression RF signals along with its unwrapp representation

Figure 5. 8 Phase shift between the $5^{\text {th }}$ RF signal pairs resulted by the quadrature method and the unwrapped version of it 86

Figure $5.9 \pi$ phase jump creation as aresult of movement from quadrant 2 to 4 (or vice versa) 87 Figure 5. 10 Real and imaginary parts of the $5^{\text {th }}$ simulated RF signal......................................... 87

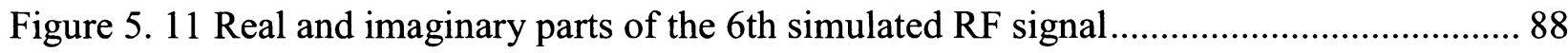
Figure 5. 12 Accumulated phase shift resulted after 5 frames, equivalent to $200 \mu \mathrm{m}$ displacement of the sample volume along with consecutive phase shifts corresponding to RF signal pairs of 1 to 4 89

Figure 5.13 Accumulated phase shift resulted after 21 frames, equivalent to $1 \mathrm{~mm}$. displacement of the sample volume after compressions 90

Figure 5.14 Unwrapped version of the accumulated phase shift, eqivalent to $1 \mathrm{~mm}$ displacement of the sample volume after compression 91 
Figure 5. 15 Phase shift between pre- and post-compression RF signals with the SNR of $10 \mathrm{~dB}$

Figure 5. 16 Phase shift between pre- and post compression RF signals with the SNR of $3 \mathrm{~dB} \ldots .$.

Figure 5. 17 Quadrature phase shift error $\left(\Delta \varepsilon_{1}\right)$ of the first RF signal pairs vs. the signal to noise ratio (SNR) 95

Figure 5. 18 Phase shift between pre- and post-compression RF signals when large amount of scatterers is assumed 96

Figure 5. 19 Quadrature phase shift error $\left(\Delta \varepsilon_{1}\right)$ of the first RF signal pairs vs. the number of scatterers $(L)$. 97

Figure 5. 20 Phase shift between pre- and post-compression RF signals with echoes' bandwidth equal to $2.25 \mathrm{MHz}$. 98

Figure 5. 21 Phase shift between pre- and post-compression RF signals with echoes' bandwidth equal to $4.2 \mathrm{MHz}$....... 99

Figure 5. 22 Quadrature phase shift error $\left(\Delta \varepsilon_{1}\right)$ of the first RF signal pairs vs. the bandwidth of the received $\mathrm{RF}$ signal $(\beta)$ 100

Figure 6. 1 Curve of the misfit norms and associated model norms. 108

Figure 6. 2 Phase of the post-compression RF signal with the SNR of $10 \mathrm{~dB}$, which is measured with different methods of quadrature, phasor and GN algorithm (with optimal regularization parameters).

Figure 6. 3 Phase shift between pre- and post-compression RF signals with the SNR of $10 \mathrm{~dB}$, measured by three methods of quadrature, phasor and GN algorithm (with optimal regularization parameters). 125

Figure 6. 4 Reconstructed phase error $\left(\varepsilon_{2}\right)$ corresponding to the post-compression RF signal with the SNR of $10 \mathrm{~dB}$ vs. different regularization parameters. 127 Figure 6. 5 Phase of the pre-compression RF signal with the SNR of $3 \mathrm{~dB}$, which is measured with three methods of quadrature, phasor and GN algorithm (with optimal regularization parameters).

Figure 6. 6 Phase shift between pre- and post-compression RF signals with the SNR of $3 \mathrm{~dB}$, measured with three different methods of quadrature, phasor and GN algorithm (with optimal regularization parameters) 
Figure 6. 7 Resonstructed phase error $\left(\varepsilon_{2}\right)$ of the pre-compression RF signal (SNR $\left.=3 \mathrm{~dB}\right) \mathrm{vs}$. different regularization parameters

Figure 6. 8 Reconstructed phase shift error and quadrature phase shift error vs. signal to noise ratio of the RF signals

Figure 6. 9 Phase shift between pre- and post-compression RF signals with the SNR of $3 \mathrm{~dB}$, measured with three different methods of quadrature, phasor, and GN algorithm (with large regularization parameters)

Figure 6. 10 Phase shift between pre- and post-compresio RF signals with the SNR of $3 \mathrm{~dB}$, measured with three methods of quadrature, phasor, and GN algorithm (with small regularization parameters)

Figure 6. 11 Phase shift between the $5^{\text {th }}$ RF signal pairs $(\mathrm{SNR}=40 \mathrm{~dB})$, measured with three methods of quadrature, phasor, and GN algorithm (with large regularization parameters) ....... 136 Figure 6.12 Phase shift between the first two RF signals (SNR $=3 \mathrm{~dB}$ ), measured with the reference and quadrature methods and reconstructed by the lowpass filter and GN algorithm . 138 Figure 7. 1 First (pre-compression) measured RF signal along with its phase information that is measured by the quadrature method.

Figure 7. 2 Second (post-compression) RF signal along with its phase information that is measured by the quadrature method

Figure 7. 3 Consecutive phase shift between pre- and post-compression RF signals, measured by the quadrature method

Figure 7. 4 Consecutive phase shift between pre- and post-compression RF signals, reconstructed by the GN algorithm (regularization parameter $=1$ ).

Figure 7. 5 Phase shift between pre- and post-compression RF signals reconstructed by the GN algorithm (regularization parametr $=50$ ). 143

Figure 7. 6 Accumulated phase shift after 5550 frames, measured by the quadrature method .. 144 Figure 7. 7 Accumulated phase shift after 5550 frames, reconstructed by the GN algorithm (regularization parameter $=100$ ) 145

Figure 7. 8 Accumulated phase shift after 5550 frames, reconstructed by the GN algorithm $($ regularization parameter $=1000)$ 146 


\section{Chapter 1}

\section{Introduction}

\subsection{Overview}

In human body, the musculature has a great deal to do with physical condition and wellbeing of an individual. For instance, an athletic injury or a muscular disease can cause a number of health related problems. As such, preventing muscle injuries and/or quickly diagnosing them in case of occurrence are of ongoing interest to medical practitioners. The motivation behind the research done for this thesis is driven by the ongoing need to better monitor and diagnose muscle disorders.

Among several symptoms, stiffer muscle is a noticeable sign commonly found in different types of muscle injuries such as muscle strain, cramps and repetitive stress injuries. Techniques of muscle stiffness measurement could prove to be a valuable resource for the study of muscular diseases and athletic injuries. Ultrasound imaging techniques can provide information about muscle stiffness. These techniques may give more precise results if used as a tool to monitor muscles.

\subsection{Problem Statement}

Ultrasound compression elastography is a relatively new imaging technique that provides strain images of soft tissues under the compression. It uses ultrasound scanners to acquire radiofrequency (RF) ultrasonic signals. In this technique, the tissue under inspection is externally, 
internally or voluntarily compressed and the amount of displacement is calculated by estimating time (or phase) shift between consecutive RF signals (A-line pairs).

This thesis research focuses on the phase shift occurred between ultrasound received RF signals which correspond to the pre- and post-compression states of a tissue under a compressive force. One technique that can be used to measure this phase shift, which can be further used to provide displacement and strain information of the tissue, is a quadrature technique. Although quadrature technique has been previously employed in ultrasound-based elastography techniques, there is a requirement to improve the accuracy of estimates from this technique, especially in the presence of measurement noise. This thesis describes certain parameters/conditions affecting the quadrature technique outcomes and develops a new algorithm to improve fully or partially incorrect phase results obtained in these circumstances. Specifically, defined algorithm, as it will be explained in this research, acts as a filter able to remove the noise and fluctuations appeared in the phase measurements.

\subsection{Objectives}

The goal of ultrasound-based elastography technique is to accurately represent displacement and strain information of a tissue under compression. In this technique, the amount of displacement of the compressed tissue is calculated by estimating the phase shift between consecutive RF signals corresponding to pre- and post-compression states of the tissue. As such, phase shift estimation needed to be exact and precise. The phase (phase shift) measurement can be conducted by the quadrature phase detection method. To achieve the goals, it is preliminary to test the quadrature method in different conditions finding the parameters affecting its outcomes. These goals are pursued by development of an algorithm with the ability to improve the incorrect phase results which are obtained in these circumstances. 
In order to obtain the aforementioned goals, several steps were taken. To begin with, a simulation had to be designed in order to allow testing both the quadrature method and designed algorithm. First, the sample volume before and after compression along with consecutive received RF signals were simulated. Then, the phase shift between consecutive frames was measured by the quadrature method, while different parameters such as signal no noise ratio, bandwidth, and the number of particles in the sample volume were changed. Finally, an algorithm was designed to reconstruct incorrect and noisy phase results. After studying in the computer simulation environment, the algorithm was implemented on the measured RF signals captured from experimental phantoms. The latter step was taken to demonstrate the effectiveness of designed algorithm on phantoms mimicking human skeletal muscles.

\subsection{Thesis Contributions}

The following is a list of the main research contributions demonstrated in this thesis:

- Developed a model-based computer simulation to create several RF signals and test the quadrature phase detection technique in different conditions.

- Used a reference approach called phasor method with which the quadrature technique can be compared and the level of its accuracy can be determined.

- Defined an inverse problem with model parameter of phase and described the associated model estimation technique known as Gauss-Newton (GN) algorithm.

- Implemented the GN algorithm on simulated and measured RF signals 


\subsection{Thesis Organization}

Chapter 1 introduces the concepts discussed throughout this thesis. Chapter 2 provides background information about human muscular system, muscle injuries, and principles of medical ultrasound imaging. In addition, it presents a discussion of the current technologies used to measure the muscle stiffness. Chapter 3 serves as a source/reference for this research. It describes the ultrasonic hardware system and data processing done in previous works. It also provides information about data files of ultrasound signals gained from experimental phantoms designed in preceding researches. Chapter 4 defines the imaging environment, computer simulation and the quadrature phase detection technique. It provides a description of the pulseecho model and outlines the procedure of RF signals' phase shift measurement. Finally, it defines a reference method by which the accuracy of the quadrature method and its outcomes can be tested. Chapter 5 represents numerical simulation results. It describes the relationship between the amount of displacement of the sample volume and the phase shift created in ultrasound received RF signals. It represents different parameters affecting the quadrature technique and its phase outcomes. Chapter 6 describes inverse problems in general while emphasizes on nonlinear category and associated solving techniques. It explains the Gauss-Newton algorithm, as a solving technique, which can be applied for ultrasound phase reconstruction. It also represents some reconstructed phase information when the GN algorithm is implemented. Chapter 7 generalizes the application of the GN algorithm to the measured RF ultrasound signals and illustrates a sample of reconstructed RF signals' phase. Chapter 8 outlines conclusions and future works. 


\section{Chapter 2}

\section{Background Review}

This chapter serves as an outline of human muscular system, muscle injuries, the physics and principles of medical ultrasound imaging, and the current technologies used to measure the human musculature stiffness mentioned in many literatures.

\subsection{Human Muscular System}

The human body contains various muscles used for a number of different purposes. Three distinct types of muscles are occurred in the human body: skeletal muscle, cardiac muscle and smooth muscle. Skeletal muscles are mainly attached to and cover the bones of the skeleton helping them to control all movements of the body. Muscles in the heart are classified as cardiac and together with skeletal muscles, are categorized as striated muscles. The primary muscle of visceral organs such as the stomach, urinary bladder, blood vessels and respiratory passages are known as smooth muscle. Skeletal muscles are the voluntary muscles while cardiac and smooth muscles are involuntary. Compare to cardiac and smooth muscles, skeletal muscles are exclusive in that they contract just in reaction to a signal generated from a motor neuron (Marieb, 1995; Silverthorn, 1998). The ultrasound imaging procedure we are studying targets the skeletal muscles. Therefore, for the rest of this chapter cardiac and smooth muscles are ignored. 


\subsubsection{Skeletal Muscle}

\subsubsection{Structure}

Skeletal muscles make up the large mass of muscles in the body, nearly $40 \%$ of total body weight. Each muscle consists of skeletal muscle tissue which itself is made up of the connective tissue, nerve tissue, and blood or vascular tissue (Silverthorn, 1998; U.S. National Cancer Institute, 2005).

Skeletal muscle tissue is the contractile tissue which comprised of a number of cells that have the ability to contract in order to produce body movements. The tissue is highly supplied with blood vessels and muscle fibers which are long and slender constitutive cells (Silverthorn, 1998). The entire skeletal muscle is surrounded with a protective connective tissue called epimysium. The groups of fibers lined up in the muscle are also enclosed with further connective tissue called perimysium. These connective tissues protect the muscle and muscle fibers allowing them to resist the contraction forces. Commonly, the epimysium and perimysium expand beyond the muscle forming a tendon which attaches muscles to the connective tissues of other muscles. Typically, a muscle is attached to bones by tendons at both ends. One of the bones remains stable while the other end moves when the muscle is contracted (Marieb, 1995; Peachey et al., 1983).

When viewed at high magnification, each muscle fiber is made up of thousands of myofibrils which are cylindrical collections of contractile proteins. Myosin and actin (also known as thick and thin filaments) are two important types of proteins. Muscles contract as a result of myosin and actin interactions. During contraction, the thin filaments pulled toward the thick filaments causing myofibrils and therefore, muscle fibers to shorten (Marieb, 1995; Peachey et al., 1983; Silverthorn, 1998). 
Axons of motor neurons meet muscles at a place called the neuromuscular junction where they convey messages from brain causing the muscle to contract and relax (Marieb, 1995; Peachey et al., 1983). Neurons (or nerve cells) are a type of cells in nervous system that consist of a cell body, a long appendage called axon, and branched processes called dendrites where the axon terminates. Dendrites are chemical transmitters from one neuron to the next neuron. Neurons are categorized as sensory and motor based on the direction in which they transmit impulses. Sensory neurons carry impulses from peripheral sense receptors to the central nervous system (CNS) while motor neurons transmit impulses from the CNS to organs such as glands and muscles causing them to contract and relax (Marieb, 1995; Silverthorn, 1998).

\subsubsection{Function}

To explain skeletal muscles' contraction, it is essential to explain the motor unit first. A motor unit is made of a motor neuron and a number of muscle fibers it innervates. If a motor neuron fires an action potential (an electrical impulse), all of the muscle fibers that it innervates will contract simultaneously, causing the action potential to be produced (Marieb, 1995; Silverthorn, 1998).

Action potential is a self-propagating electrical potential difference that is produced across the membrane of nerve or muscle cells when they are adequately stimulated. It reverses the membrane potential from about -70 (resting potential) to $+40 \mathrm{mV}$. This electrical impulse is carried down the nerve through a series of action potentials (Lawrence, 2008; Matthews, 2003; Silverthorn, 1998).

As the nerve fiber branches, the action potential travels down each branch. In turn, the connected skeletal muscle fibers will be stimulated and an action potential will be produced on 
their membrane. In this way, the action potential spreads along the muscle fibers. As more nerve branches and additional fibers are activated, the action potential spreads over the entire muscle. Upon activation, the muscle contracts (Marieb, 1995).

Almost all body movements are due to the skeletal muscle contraction. Joints, bones, and skeletal muscles act together to produces obvious movements such as walking and running. Some other important functions in the body such as posture control, joint stability, and heat production are also performed by muscle contraction. An important by-product of muscle metabolism is heat which maintains body temperatures. About 85 percent of body heat is produced by the muscle contraction (Plowman \& Smith, 2007).

\subsubsection{Muscle Injury}

Muscle injuries are certain conditions that affect the skeletal muscles in response to the external forces. For example, accidents or overuse can cause muscles to be strained which in turn, causing them to swell, bruise or even tear. Muscle injuries vary based on the level of severity. They affect movement and functionality of the injured area differently. Appropriate treatment will also differ based on the area that is injured and the degree of the injury.

By sliding against each other, shortening and lengthening, the muscle fibers facilitate movement of skeletal joints. However, if these fibers experience stress, they may respond to great pressures by tearing, abruptly contracting, becoming rigid and other effects.

Muscle injuries have a variety of signs some of which are visible while others may not be instantly noticeable. It can cause inflammation and tenderness in the affected area, or result in contusion and bleeding if the injury is severe. Pain can also be felt by moving the injured muscle. A doctor can set a detailed physical test to determine the degree of the injury. Common types of 
muscle injuries are muscle strain, muscle cramps and repetitive strain injuries which are briefly described below.

\subsubsection{Muscle Strain}

Strain injury is the result of excessive muscle stretch or powerful muscle contractions which causes the muscle fibers to tear. Muscle strain is the most common injury in sports. It usually occurs as a result of activities that force the muscle to tighten. The muscle is strained either if it is not suitably stretched or warmed up before the activity, or if it is already injured and not recovered yet. Lengthening the muscle during muscle contraction, which is known as eccentric contraction, can more likely cause the muscle to strain (Garrett, 1996; Garrett, 1990; Walker, 2007). Usual symptoms of a strain are regional pain, stiffness, and bruising around the strained muscle. As a treatment, four steps commonly known as R.I.C.E are taken.

- Rest: all unnecessary activities, especially those result in pain, are recommended to be stopped.

- Ice: ice which can restrict blood flow to the injury site should be applied. Icing should be less than 10-15 minutes and it should be done indirectly by placing a layer of fabric between the ice and the injury.

- Compression: the strained area is suggested to be wrapped.

- Elevation: the strained area is better to be kept as close to the level of the heart as is easily possible.

In order to decrease the instant inflammation and lessen pain, this immediate treatment is often accompanied by the use of nonsteroidal anti-inflammatory drugs such as ibuprofen (Garrett, 1990; Järvinen et al., 2007). 


\subsubsection{Muscle Cramps}

Cramps are hurtful and annoying muscle injuries caused by sustained contraction or over shortening of skeletal muscles (Ylinen et al., 2008; Jones et al., 1990; Silverthorn, 1998). Muscle fatigue and body's depletion of essential electrolytes (e.g., potassium, calcium or sodium chloride) are the common causes of skeletal muscle cramps (Marieb, 1995; Hoeger et al., 2008; Larson-Meyer, 2007; Jankovic et al., 2007). Hyperflexion, hypoxia (Lance-Adams syndrome) and exposure to large changes in temperature, or dehydration are other causes of cramping. It can also occur as a side effect of medications specially tablets and pills which cause the elimination of fluid from the body resulting in dehydration. Muscle cramps may also be occurred due to pregnancy, kidney disease, thyroid disease, or many other complications (Benjamin et al., 2005; Jankovic et al., 2007).

Skeletal muscles function as opposing pairs. i.e., when one muscle contracts the opposing muscle in the pair has to be relaxed. Cramps may occur when muscles cannot relax appropriately. It happens when myosin and actin filaments are kept attached to each other. In skeletal muscle, myosin filaments need to obtain enough energy and magnesium in order to detach from the muscle and allow relaxation. Insufficient quantities of either of these causes the myosin remains attached to the actin and therefore, cause the muscle cramps (U.S. National Cancer Institute, 2005).

A cramp is characteristically painful and severe. Severe cramps which are accompanied with soreness and swelling can continue up to several days after the cramp has gradually lessened (Larson-Meyer, 2007). During the cramping, the knotted muscle might feel very firm.

Some sort of muscle cramps such as those occurred due to fatigue can be treated by stretching and massage, it can be painful though (Hoeger, et al., 2008; Jones et al., 1990; Marieb, 1995). In 
some cases ice packs can help relaxing the tense muscles while heating pads can be effective in other cases (Järvinen et al., 2007). If cramp is occurred due to dehydration, then fluids with electrolytes such as sports drinks can balance the fluid loss (Benjamin et al., 2005; Jankovic et al., 2007). Those muscle cramps occurred as a side effect of certain medications can be treated by adjusting the dosages or changing the medication. Treating the illness is the solution when the cramps are caused by an illness.

\subsubsection{Repetitive Stress Injury}

Repetitive strain injury (RSI) is the musculoskeletal and nervous systems' injury which may occur as a result of repetitive tasks, forceful actions (Helliwell, 1992; Sommerich, 1993), mechanical compression (e.g., pressing against hard surfaces), continual or uncomfortable positions, or muscle fatigue. These injuries happen when excessive pressure is applied on a part of the body, resulting in inflammation (pain and swelling), toughness of the muscles, or tissue damage. Those individuals who are involved with repetitive tasks, such as writing, playing musical instruments, computer work, and other cyclic tasks are more affected by RSIs. Over a long time, repetitive stress injuries can cause temporary or permanent damages to different tissues such as muscles, nerves, tendons and ligaments (Typer, 1994; Fry, 1986; Solly, 2007).

Symptoms of RSIs include tingling, numbness, pain and stiffness in the affected area, feelings of weakness or fatigue and clicking sensation (Typer, 1994). Reducing or stopping the motions or activities that cause symptoms are the usual treatment of RSIs. Taking breaks to give the injured area time to rest, doing stretching and relaxation exercises, applying ice to the affected area to lessen inflammation, and using medications such as pain relievers are other types of treatments (Järvinen et al., 2007). Ergonomic adjustments of the workstation are also 
recommended since prolonged and repetitive work at this area can create RSIs, particularly of hand and wrist (Aarås, 1994).

\subsubsection{Diagnosis and Monitoring by Ultrasound}

Muscle injury and common examples of it were described above. Statistics show that many people experience muscle injuries. For instance:

- 30 to $80 \%$ of those people carrying out repetitive tasks without work breaks are involved with some sort of RSIs.

- Within a three year period, $60 \%$ of the workforces were reporting symptoms of muscle cramp or pain due to utilizing the new technology (Typer, 1994).

In some cases, quickly diagnosis is necessary while delay would lead patient to more serious problems. For example, any warning signs of RSIs even symptoms look as they come and go shouldn't be ignored or they may conduct to permanent or serious problems. If RSIs are not treated, they can prevent patient from doing simple everyday tasks such as doing sports, playing music, and participating in other favorite activities.

Based on these facts, muscle injuries should be diagnosed and monitored properly and treated in an appropriate manner. The diagnosis of muscle injuries is usually made by history and physical examination. The therapist will take the patient medical history and ask a number of questions to figure out the problem.

Ultrasound is a good monitoring tool since it can represent information about the stiffness of the muscle. As stated earlier, besides pain and soreness, stiffer muscle is a noticeable symptom commonly observed in an injured muscle. The amount of stiffness can be measured and revealed 
by the means of ultrasound techniques. Ultrasound may in fact, give more accurate results if it is used as a tool for monitoring diseased muscles.

\subsection{Ultrasound Imaging}

A basic ultrasound imaging system contains a transducer and associated electronics such as a display. It utilizes electrical-to-acoustical transducers to produce pulses of high frequency sound. These pulses are transmitted through a patient's body and reflected back at organ boundaries and complex tissues. The transducer is again used to detect these created echoes. The ultrasound imaging system processes the echoes received by the transducer and provides a grayscale image of the body structure on a display. Each point in the image corresponds to the anatomic location of an echo-generating structure (Prince \& Links, 2006).

Ultrasound itself is sound with frequencies higher than the highest frequency that can be heard by humans. Any sound above about $20 \mathrm{KHz}$ is known as ultrasound. Medical ultrasound systems operate at much higher frequencies, typically between 1 and $10 \mathrm{MHz}$ (Prince \& Links, 2006). In the following section, the basic physics behind the ultrasound, ultrasonic transducer, and the most common ultrasound imaging modalities are described.

\subsubsection{Ultrasound Physics}

Ultrasound is an acoustical pressure wave that propagates through materials via compression and expansion of the material. These waves can have different patterns. As an acoustic wave propagates, small particles of the material move back and forth in order to generate the compression and expansion of the acoustic wave. In medical ultrasound, where waves propagate only in soft tissues, these particles move back and forth in the same direction that the acoustic 
wave is traveling. When this happens, the acoustic wave is called a longitudinal wave or compressional wave. Harder materials also support shear waves or transient waves, in which the particles move at the right angles to the direction of acoustic wave propagation (Prince \& Links, 2006).

Ultrasound imaging technology is conducted by the physics of sound propagation. The first important property of sound, which is the acoustic velocity, and some physical properties of the interaction of ultrasound with tissue are outlined in the following subsections (Hedrick, Hykes, \& Starchman, 2005).

\subsubsection{Acoustic Velocity}

Acoustic velocity is the speed of sound transmission in a medium, which is normally represented by $c$. The medium through which the sound is travelling determines the acoustic velocity. As such, the speed of sound propagation can vary while it is travelling through tissue (Hedrick et al., 2005).

Density (mass of the medium per unit volume) and compressibility (the fractional decrease in volume as a result of pressure application) are two physical characteristics of a medium that can affect the acoustic velocity. An increase in density or compressibility will oppose the sound propagation through the medium. Numerically, the acoustic velocity is inversely proportional to the square root of both the density and compressibility of the medium. However, a variation in density is often coupled with a greater and opposing variation in compressibility. Therefore, compressibility is considered as the dominant factor. In overall, the effect is summarized by the statement that, as the density increases the velocity of sound through a medium increases. 
Combining compressibility $(\kappa)$ and density $(\rho)$ into one equation, the acoustic velocity $(c)$ for a particular medium is defined by

$$
c=\frac{1}{\sqrt{h \rho}}
$$

The velocity of sound in air, bone and soft tissues is 330,4080 and around $1500 \mathrm{~m} / \mathrm{s}$, respectively (Hedrick et al., 2005).

\subsubsection{Attenuation}

When an ultrasound wave is transmitted into the body, it interacts with tissues based on their characteristics. The result of these interactions is recorded for diagnosis in the form of reflected ultrasound waves (echoes) (Bhargava, 2002). Reflection, scattering, refraction, diffraction and absorption are the most common interactions. By redirecting the energy of the ultrasonic beam, these interactions, which are termed as attenuation, decrease the amplitude and intensity of the beam (Hedrick et al., 2005). Attenuation mostly includes the reflection and scattering of the sound as it crosses tissue boundaries or heterogeneous tissues, and the absorption (conversion of ultrasonic energy to heat) of the sound as it travels. From theses interactions, absorption is the dominant factor contributing to the ultrasound attenuation in soft tissues (Kremkau, 2006).

\subsubsection{Acoustic Impedance}

Acoustic impedance is the product of density and acoustic velocity. It measures the resistance to sound traveling through the medium (Hedrick et al., 2005). When the ultrasound wave crosses an interface (e.g. the boundary between different tissue types), a portion of the incident will be 
passed to the second tissue while the rest is reflected back to the first tissue due to the difference in tissues' acoustic impedances. The impedance ratio between the two tissues specifies how much of the incident sound wave is reflected back to the first tissue (Kremkau, 2006). Visualization of soft tissue structures with an ultrasonic beam is the result of this change in acoustic impedance at a biological interface (Hedrick et al., 2005).

\subsubsection{Reflection}

Ultrasound images are created from echoes generated by reflections of ultrasound waves at tissue interfaces and by scattering resulted from small irregularities or inhomogeneities within the tissue.

In diagnostic ultrasound, reflection is the major interaction. If a sound beam incidence is conducted to a smooth interface larger than the beam width, a portion of it will be reflected toward the sound source due to different impedances. These large interfaces are called specular reflectors which constitute the major organ outlines in ultrasound images. At these boundaries, the angle of sound beam incidence and reflection are the same. These angles are defined with respect to a line drawn perpendicular to the interface surface (Figure 2.1). The maximum detection of reflected echo can be obtained by orienting the transducer in a way that the generated sound beam hits the interface perpendicularly, and in return, the reflected wave passes the same path back toward the transducer (Figure 2.2) (Hedrick et al., 2005). 


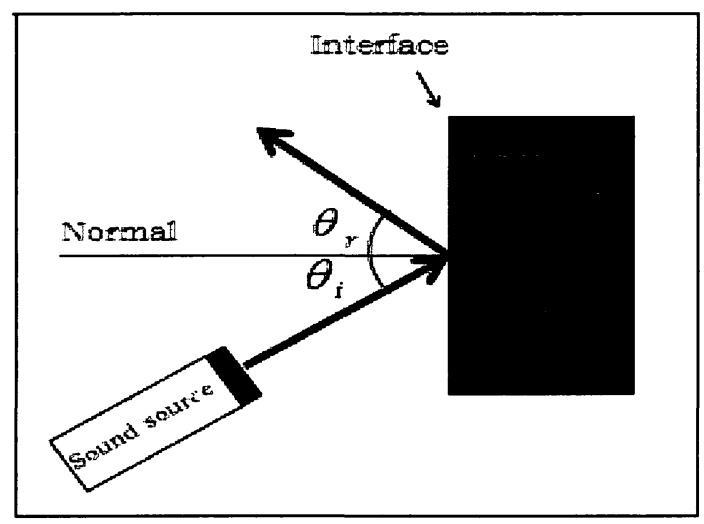

Figure 2.1 Reflection caused by a sound wave striking a specular reflector at an angle; the resulting angle of relection is equal to the angle of incidence (Hedrick et al., 2005).

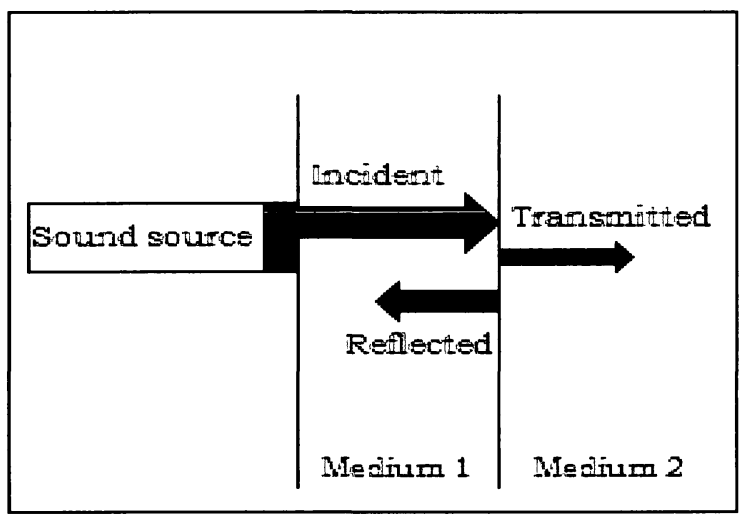

Figure 2.2 Reflection caused by a sound wave striking a specular reflector at normal incidence; the acoustic impedances of the media that compose the interface determine the relative intensities of the transmitted and reflected waves (Hedrick et al., 2005).

\subsubsection{Scattering}

Another important interaction between ultrasound and tissue is scattering, or nonspecular reflection. Internal pattern of organs in the image is provided as a result of scattering. Scattering occurs at the small boundaries with physical dimensions around the size of wavelength or even smaller. A boundary (interface) of this size is often called as a scatterer. Each scatterer acts as a 
new separate sound source and reflects the sound in all directions independent of the direction of arriving incident. The scattered ultrasound has much weaker intensity than specular reflection (Hedrick et al., 2005). Figure 2.3 demonstrates the nonspecular reflection.

An ultrasound pulse simultaneously encounters many scatterers at different positions in its path causing several echoes to be generated. During scanning, the resulted echoes interact constructively or destructively with each other making a displayed grainy appearance that illustrates the interference pattern of the scatterer distribution scanned. This phenomenon is known as acoustic speckle and echoes generated at the position of scatterers are referred to as speckle echoes (Kremkau, 2006).

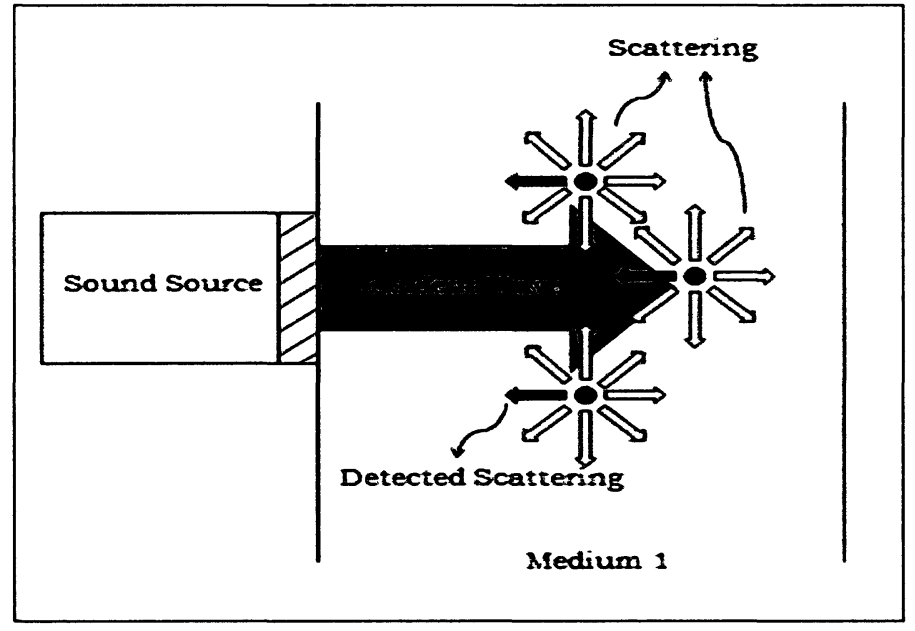

Figure 2.3 The incident wave is simultaneously encounters many scatterers at any location in its travel and reflected back resulting in nonspecular reflections; only the scatterings that travel in similar path but in opposite direction of incident wave are detected by the ultrasound transducer.

\subsubsection{Ultrasonic Transducer Elements and Probes}

An ultrasound transducer is an instrument that converts electrical pulses into ultrasonic pulses, and conversely, ultrasonic echoes into electrical echoes (Hoskins, Thrush, Martin, \& 
Whittingam, 2003). The number of transducers and the way they are arranged in an ultrasound probe varies depending on the intended application. All ultrasonic probes, however, contain at least one transducer component made up of typically a piezoelectric material (lead zirconate titanate or PZT), matching layer(s) and a backing layer (Hoskins et al., 2003). The piezoelectric material exhibits a strong piezoelectric effect in a way that an applied electric field produces mechanical displacement, which consequently causes an acoustic wave. It also satisfies the reciprocal property that a mechanical displacement induces an electric potential which can sense an acoustic wave (Prince \& Links, 2006). An example of ultrasonic transducer is shown in Figure 2.4. As it can be seen, matching layers are located at the face of transducer after PZT and just before the tissue that aimed to be imaged. These layers are used to match the impedances of PZT and the human tissue. If not employed, the majority of sound wave will be reflected back to the tissue at the PZT-tissue boundary due to the high impedance of PZT (Hedrick et al., 2005). When the sound wave is initiated, it moves in both forward and backward directions into the tissue and the PZT material, respectively. The backing layers are employed to remove the backward travelling wave which hits the back face of transducer and reflects toward the tissue. This backward travelling wave should be removed before it reaches the tissue boundary since it is not obtained from within the tissue aimed to be imaged (Prince \& Links, 2006). Therefore, the backing layer should have an acoustic impedance close to that of the PZT material so that the wave completely transmits from PZT to backing layer. Besides, it should have a high absorption coefficient to absorb the backward propagating wave preventing it from entering the PZT material again (Hedrick et al., 2005). 


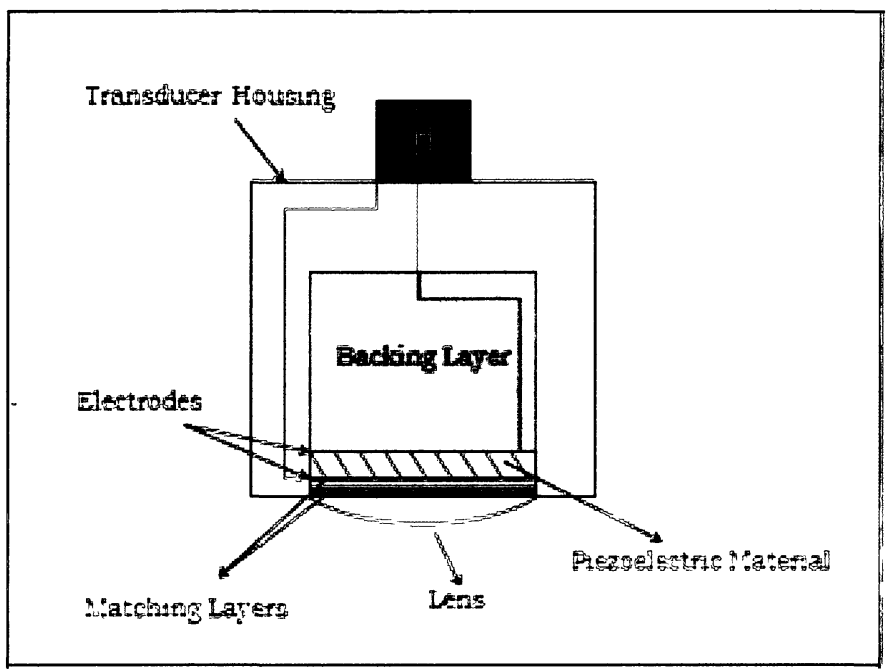

Figure 2.4 Construction of a single-element transducer. Reproduced from (UKessays.com)

\subsubsection{Imaging Modalities}

Ultrasound imaging can be used for a number of different applications which are all involved with the principles of sound propagation. Currently, various imaging modalities are used to provide a helpful tool to practitioners. The most common ultrasound imaging modalities are Amode, B-mode and M-mode which are explained in the following subsections.

\subsubsection{A-Mode}

A-mode or amplitude mode is the simplest ultrasound imaging mode. In A-mode imaging, spatial information is one-dimensional since pulses are transmitted in only one direction. In this mode, the amplified amplitudes of the received echoes are plotted on the vertical axis of the oscilloscope's screen as a function of the transient time represented on the horizontal axis (Mantke \& Peitz, 2007). In order to create and display a sequence of these signals, the transducer is repeatedly fired. The time between successive firings (excitations) of the transducer is called 
the pulse repetition period and is shown by $\left(T_{R}\right)$ (Prince \& Links, 2006). An example of an Amode scan and the resulting image is represented below in Figure 2.5.
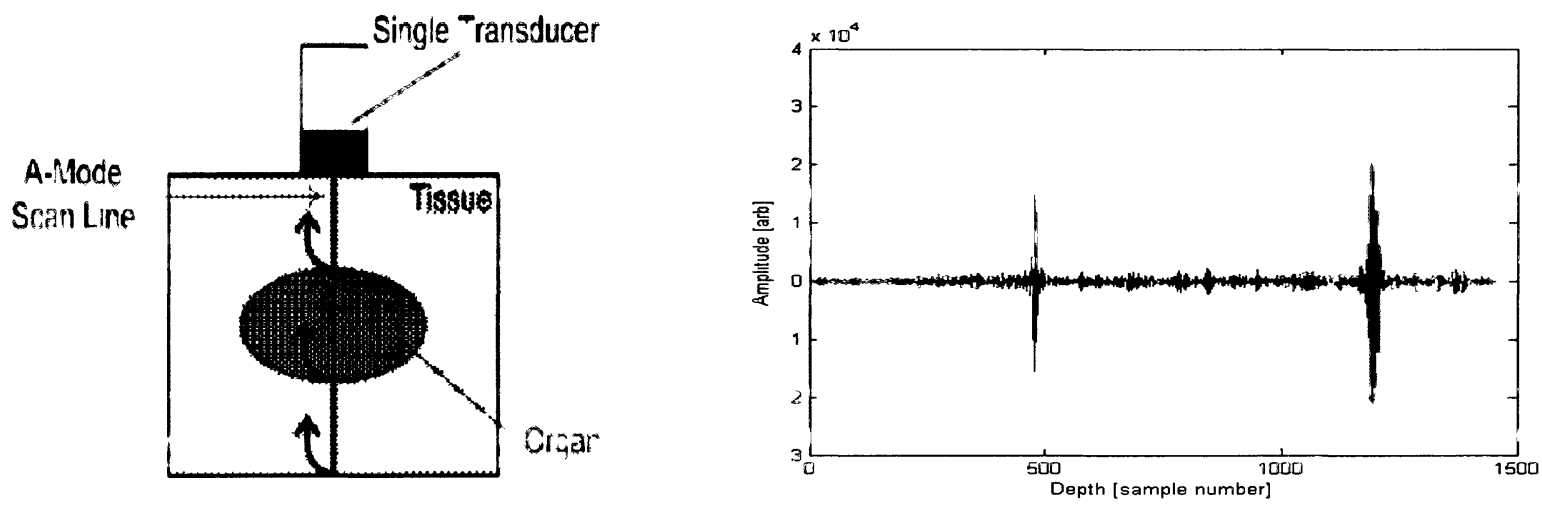

Figure 2.5 An example of A-mode scans (left) and the resulted image (right). Reproduced from (Silver, 2009)

\subsubsection{B-Mode}

In B-mode or brightness mode imaging, the two-dimensional spatial information is provided. In this mode instead of deflections along the vertical axis, the amplitudes of echoes are plotted as pixel intensity (brightness) which is graded by means of a gray-scale. The generated echoes will yield a line of pixels the distance of which will represent the transducer distance to the various interfaces. The direction of the pulse-echo train is changed mechanically such that it can provide a cross-sectional image of a part of body (Mantke \& Peitz, 2007). Traditionally, this was achieved by stirring or rotating the transducer laterally along the skin surface while A-mode scan lines were captured. However, newly designed array ultrasonic probes contain a number of transducers which can create multiple A-mode scan lines (Hoskins et al., 2003). On the screen, lines of pixels are arranged in parallel producing a two-dimensional image of the viewing plane 
(Mantke \& Peitz, 2007). More specifically, the resulted image represents both lateral (perpendicular to beam direction) and axial (parallel to beam direction) information (Hoskins et al., 2003). Figure 2.6 represents this type of scanning.
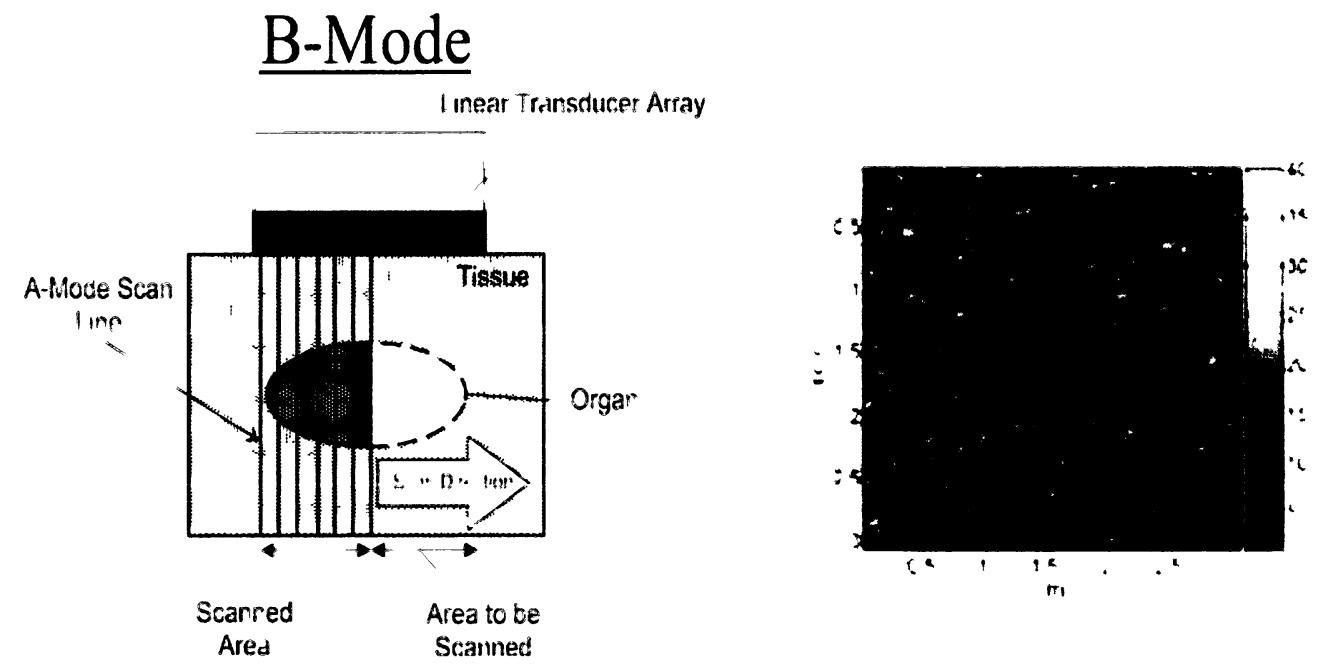

Figure 2.6 An example of B-mode scans (left) and the resulted image (right). Reproduced from (Hoskins et al., 2003; Tsui et al., 2007).

\subsubsection{M-Mode}

The M-mode, also known as motion mode, is similar to the A-mode in that it provides one dimensional spatial information. In this mode, the direction of pulse-echo train remains fixed providing a single straight, line of sight of any motion taking place in a direction towards or away from the transducer. Similar to B-mode scanning, the amplitudes of received echoes are plotted by pixel brightness. Vertical and horizontal axis of the screen represents the distance of the echoes from the transducer and the transient time, respectively. M-mode imaging is particularly used when examination of a rapid interface motion such as cardiac valves motion is intended (Mantke \& Peitz, 2007). 


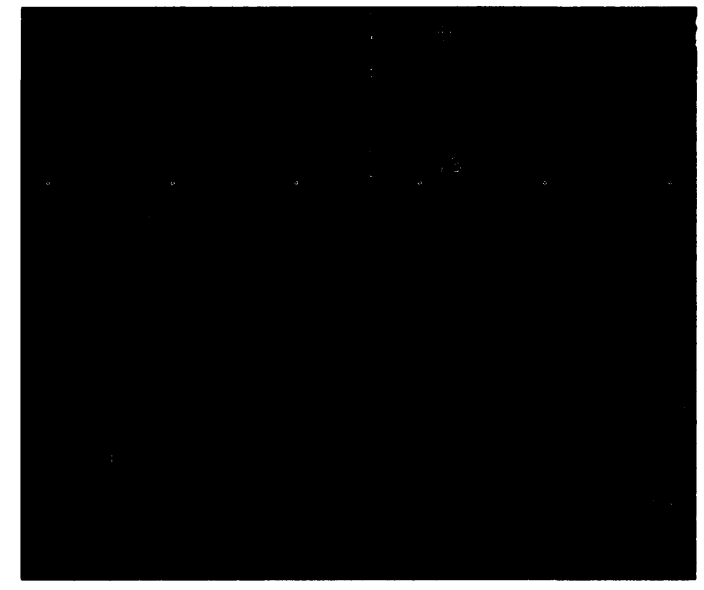

2.7 An example of M-mode image. Reproduced from (Mayo \& Doelken, 2009)

The basic principles of ultrasound as a medical imaging modality have been explained above. As an imaging modality, ultrasound can measure various quantities. It may also be used as technique to study muscle. Specifically, it can be used to track muscle motion and to measure muscle structure (Hodges et al., 2003; Farron et al., 2009; Larsson et al., 2009; Neves et al., 2007). Ultrasound techniques are applied to a variety of applications. A relatively new application is elastography which is an area where the ultrasound is used for muscle strain/structure measurements.

\subsection{Elastography: A Technique for muscle strain measurement}

Elastography is a relatively new imaging technique that provides strain images of soft tissues under the compression. Strain indicates the relative deformation in such a way that stiff tissue shows less strain compare to the softer tissue while they are both under identical force. Strain measurement is important since it is related to the stiffness and as discussed earlier, many 
muscular disorders are often correlated with changes in tissues' stiffness. New quantitative information about the stiffness of the tissues that would not be achievable with current imaging modalities can be provided by elastography (Souchon, 2007; Peng et al., 2006; Bae et al., 2007). Elastography can be done by different imaging techniques such as ultrasound, magnetic resonance imaging, optical coherence tomography, and X-ray computed tomography (Varghese, 2009). In this thesis, the focus is on ultrasound-based elastography since it is more commonly used for clinical elasticity imaging (Greenleaf, Fatemi, \& Insana, 2003). The following subsection describes strain and different types of ultrasound elastography.

\subsubsection{Strain}

The term strained described in this section is a mechanical property of the tissue and is different from muscle strain described as one type of muscle injuries.

Strain is defined as the deformation of an object, normalized to its initial shape. The only possible deformation for a one-dimensional object (i.e. an infinitesimally thin bar) is lengthening or shortening. This is illustrated in Figure (2.8). Strain, for which the symbol $s$ is used, can thus, be written as (Ophir, 1997; D’hooge, 2000):

$$
s=\frac{\ell-\ell_{0}}{\ell_{0}}
$$

where $\ell$ is the length of the object after deformation and $\ell_{0}$ is its initial length. It is a dimensionless quantity since it is the change in length relative to its initial length. By lengthening an object, the strain will become positive whereas shortening causes the object to have a negative strain (D'hooge, 2000). 


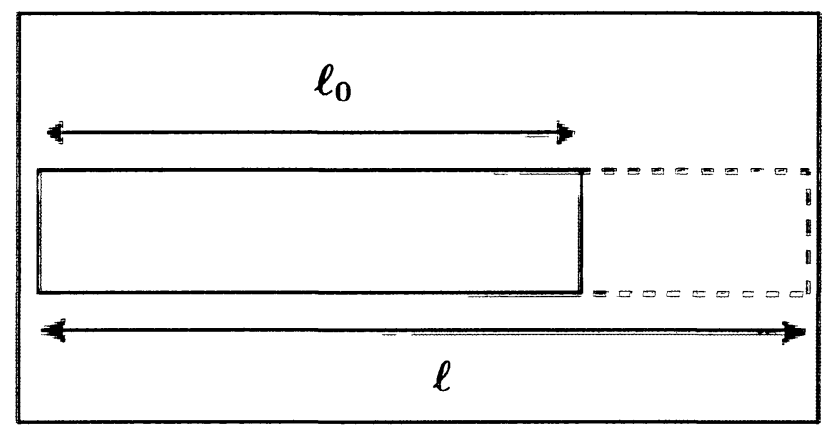

Figure 2.8 Deformation of a one-dimentional object which is limited to lenghtening and shortening (D'hooge, 2000).

\subsubsection{Ultrasound-based Elastography}

Ultrasound elastography falls into three main groups: compression elastography (strain imaging), transient elastography, and vibration sonoelastography (Souchon, 2007). The focus of this research is on compression elastography type. This choice was made because in this methodology the process of strain measurement involves no external apparatus such as a mechanical vibrator to produce shear waves for shear wave elastography (Greenleaf et al, 2003). In the following sections, the three different types of ultrasound-based elastography are explained.

\subsubsection{Compression Elastography}

Compression elastography is an imaging technique that is based on the static deformation of an elastic material (Gao, Parker, Lerner, \& Levinson, 1996). It is based on radio-frequency (RF) ultrasonic signals captured by ultrasound scanners.

In this technique, the tissue under inspection is compressed as a result of force application causing RF signals being compressed and displaced. Each particle in the tissue along the 
direction of ultrasonic beam propagation is displaced to some extent. The local displacement of each particle induces a shift in the time domain of the corresponding echo in the backscattered signal as a result of the variation made in the time of flight. The resulted time shift is directly proportional to the local displacement and is given by the variation in the round-trip time (RTT) of flight of the ultrasonic signal by (D'hooge, 2000; Souchon, 2007).

$$
\tau(t)=\frac{2 d(t)}{c}
$$

where $c$ is the acoustic velocity, which is assumed to be constant, $d(t)$ is the local displacement of the tissue at a depth corresponding to time $t$ along the ultrasound beam direction and $\tau(t)$ is the corresponding time shift induced at time $t$, in the domain of the echo generated at this time. This process is described in Figure 2.9.

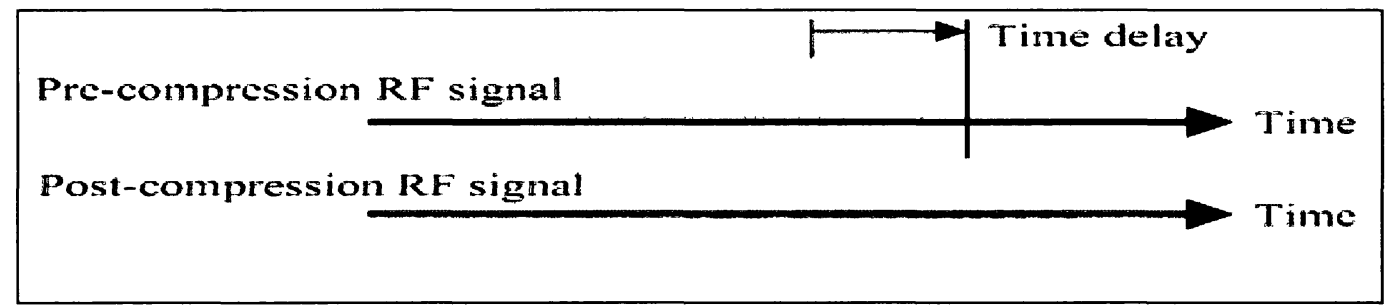

Figure 2.9 Pre- and post-compression RF signals; displacement of the tissue (particles) induces time shifts. Reproduced from (Souchon, 2007)

\subsection{Time Shift Estimation:}

In order to estimate time shift $\tau(t)$, pre- and post compression A-line pairs (RF signal pairs) are acquired. An A-line pair consists of the original A-line which is acquired when the tissue 
is slightly pre compressed by the ultrasound probe to make sure a good contact is established, and a compressed A-line which is obtained when the tissue is axially compressed an additional increment (usually about $1 \%$ of the target length). Compressive stress causes the post compression A-line to become shorter than the pre compression one. Therefore, in order to have the same length, the post compression A-line is zero-padded (Gao et al., 1996; Hein et al., 1993). A lines are then, divided into short segments called windows, and the time delay $\tau(t)$ between corresponding windows is estimated via cross-correlation process, which is a process that quantitatively measures the similarity between two delayed signals. The location where the cross-correlation function takes its maximum value is the estimate of the time shift between the two windows (Gao et al., 1996; Souchon, 2007). In the following, this process is described mathematically

\subsection{Cross Correlation Function}

The cross correlation function is defined as (Greenleaf et al., 2003; D'hooge, 2000)

$$
r(\hat{\tau}(t))=\max _{\tau} \int_{-\infty}^{+\infty} \zeta\left(t_{0}+\tau\right) w\left(t_{0}-t+\tau\right) \cdot \zeta^{\prime}\left(t_{0}\right) w\left(t_{0}-t\right) d t_{0}
$$

where $\zeta(t)$ and $\zeta^{\prime}(t)$ are the pre- and post-compression RF signals (A-line pair), respectively, $t_{0}$ is an integration variable, and $t$ is the time corresponding to the location of the window. In this integration, $\zeta(t)$ is multiplied by a window function $w(t)$ and cross correlated with $\zeta^{\prime}(t)$, which is windowed similarly.

After estimating the times shifts, local displacements of different particles in the tissue can be estimated as follows 


$$
\hat{d}(t)=\frac{c \hat{\tau}(t)}{2}
$$

where $c$ is the speed of sound and $\hat{\tau}(t)$ and $\hat{d}(t)$ are the estimated time shift and local displacement of the tissue at a point corresponding to the time $t$, respectively (Greenleaf et al., 2003). Symbol` is used to indicate the estimated values.

The time scale is presumed to be relative to the face of the transducer. Thus, at the beginning of an A-line pair, the relative shift of the signal is very small, while it becomes larger towards the end. In general, time shift in the compressed A-line relative to the uncompressed A-line increases from 0 to a $\frac{2 \hat{d}(t)_{\max }}{c}$. If the elastic modulus differs somewhere along the line and the tissue becomes stiffer, the amount of local displacement at that point will become small and thus, little or no increase will be observed in the time shift of related windows (Gao et al., 1996; Ophir et al, 1991; Hein et al., 1993).

Estimation of time delays $(\tau(t))$ is one approach that is normally used to further estimate the local displacements $(d(t))$ and strain. Instead of time shift estimation, phase shift measurements can also be used to further estimate the local displacement. Induced time shift changes the phase information of A-lines causing a phase shift to be created between the pre- and post-compression A-lines at different points in tissue. Measurement of this phase shift is of interest in thesis. In chapter 4, this approach will be discussed in detail. The following section provides an explanation of the strain estimation in this technique. 


\subsection{Strain Estimation}

Axial strain $s$ is given by the gradient of the axial displacement in the axial direction $z$, where $z=\frac{c t}{2}$ (Souchon, 2007; Greenleaf et al., 2003).

$$
s=\frac{\partial \hat{d}(t)}{\partial z}=\frac{2}{c} \frac{\partial \hat{d}(t)}{\partial t}
$$

In Eq. (2.6), $\hat{d}(t)$ is the estimated local displacement at a depth corresponding to time $t$ and $\partial \hat{d}(t)$ is the difference between local displacements of any two windows. $\partial z$ is the original difference between the corresponding two windows which is equivalent to $\partial t$ in time domain. If the strain is aimed to be calculated with respect to the tissue depth then, the first ratio shown in Eq. (2.6) should be applied. If, however, it is intended to be estimated with respect to the time domain then, the second ratio, where the coefficient $\frac{2}{c}$ is incorporated, will be used.

Particularly, the gradient is obtained using two consecutive windows separated by a window shift $\Delta t_{i}$ as (Greenleaf et al., 2003; Ophir et al, 1991)

$$
s_{\imath}=\frac{2}{c} \frac{\hat{d}\left(t_{i+1}\right)-\hat{d}\left(t_{i}\right)}{\Delta t_{i}}
$$

where $\hat{d}\left(t_{i}\right)$ is the amount of displacement of the $i^{\text {th }}(i=1$ to $K)$ window and $s_{i}$ is the strain estimate for segment pair i. $\Delta t_{1}$ is the original difference between the two consecutive windows of $i$ and $i+1$. The strain at first depth increment, when the first two consecutive windows are considered, is shown in Figure 2.10. 


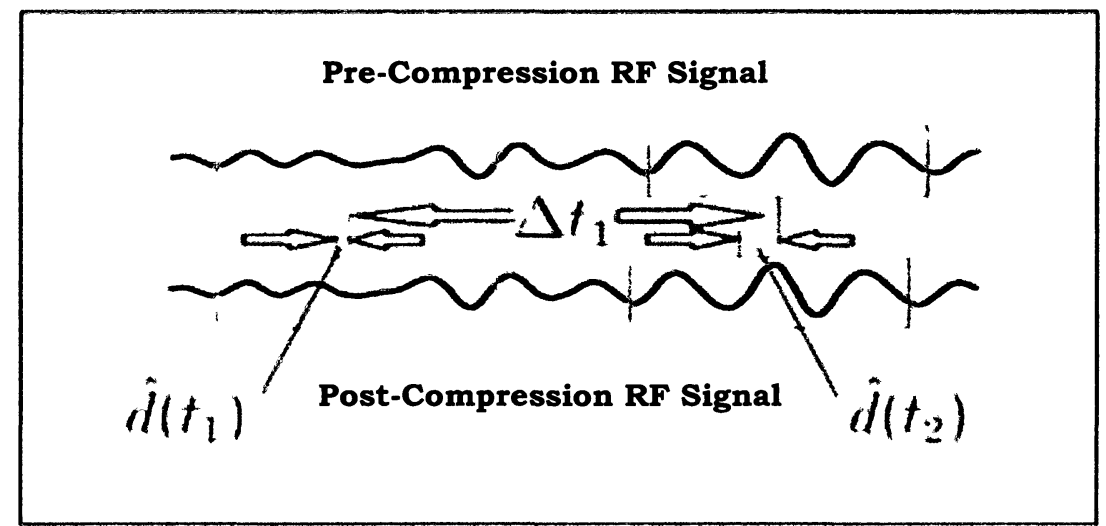

Figure 2.10 Strain is estimated using the local displacements in the first two consecutive windows (Souchon, 2007).

After processing one A-line pair, assuming that the A-lines are segmented into $K$ windows, a number of displacements, $\hat{d}\left(t_{1}\right)$ through $\hat{d}\left(t_{K}\right)$. corresponding to the $K$ windows will be acquired (Greenleaf et al., 2003; Ophir et al, 1991). Then, the related strain profile is represented as a onedimensional graph which demonstrates the strain as a function of depth (or corresponding time) in the tissue. Similarly, for all A-line pairs the process can be repeated and an array of strain data called strain image (or elastogram) can be obtained (Ophir et al, 1991; Ophir et al, 1997; Souchon, 2007).

The strain needs not necessarily be estimated by the two consecutive windows. It can be estimated by any two different points in the depth like $a$ and $b$ in the following way:

$$
s_{a, b}=\frac{2}{c} \frac{\hat{d}\left(t_{a}\right)-\hat{d}\left(t_{b}\right)}{t_{a}-t_{b}}
$$


where $\hat{d}\left(t_{a}\right)$ and $\hat{d}\left(t_{b}\right)$ are the local displacements of windows corresponding to time $t_{a}$ and $t_{b}$. respectively.

In the following, it is tried to show the concept of strain and its relationship to the stiffness of a tissue under compression. As explained earlier, time shift of the compressed A-line relative to the uncompressed A-line increases from 0 to $\frac{2 d(t)_{\max }}{c}$. Consequently, the amount of local displacement of the particles along the post-compression RF-signal increases linearly from 0 to $\frac{c \tau(t)_{\max }}{2}$. A typical plot of local displacements of particles with respect to their position (depth) in a normal tissue under compression is shown in Figure 2.11. Corresponding strain curve is obtained by taking the gradient of the displacement curve.

Displacement $(d(z))$

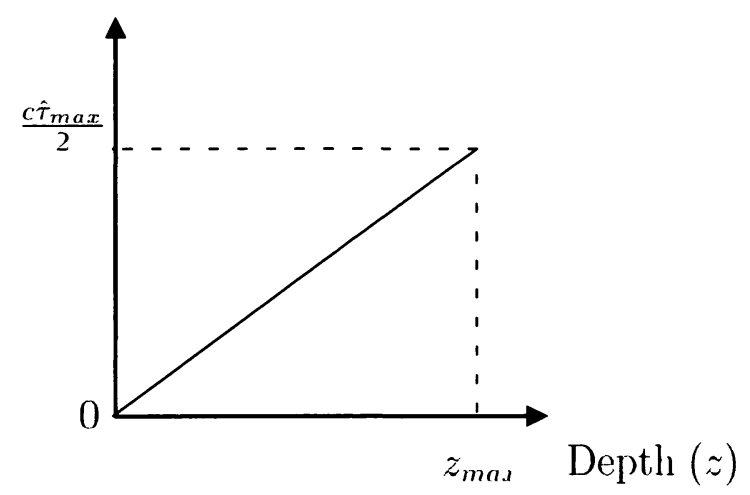

Strain $(s)$

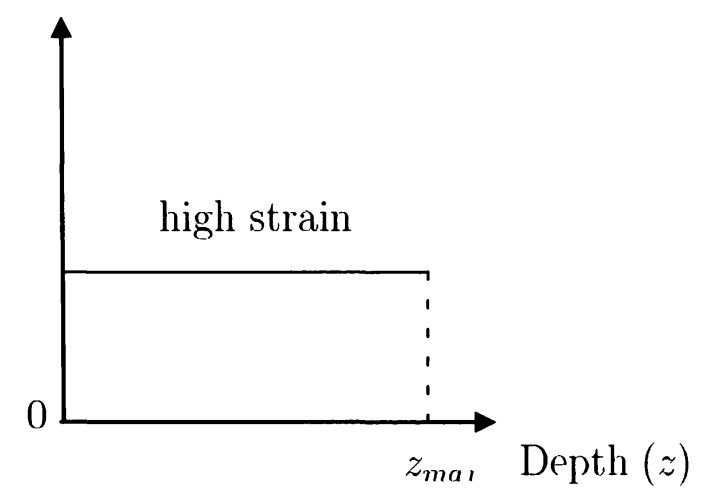

Figure 2.11 Curve of the local displacements of the particles with respect to their position (depth) in a compressed normal tissue (left ) and corresponding strain curve (right).

If however, the tissue becomes stiffer, similar to what happens to an injured muscle, then the slope of displacement curve represented in Figure 2.11 will reduced and the strain will become 
smaller (Figure 2.12). Lower strain indicates that the part of body under examination has become stiffer.
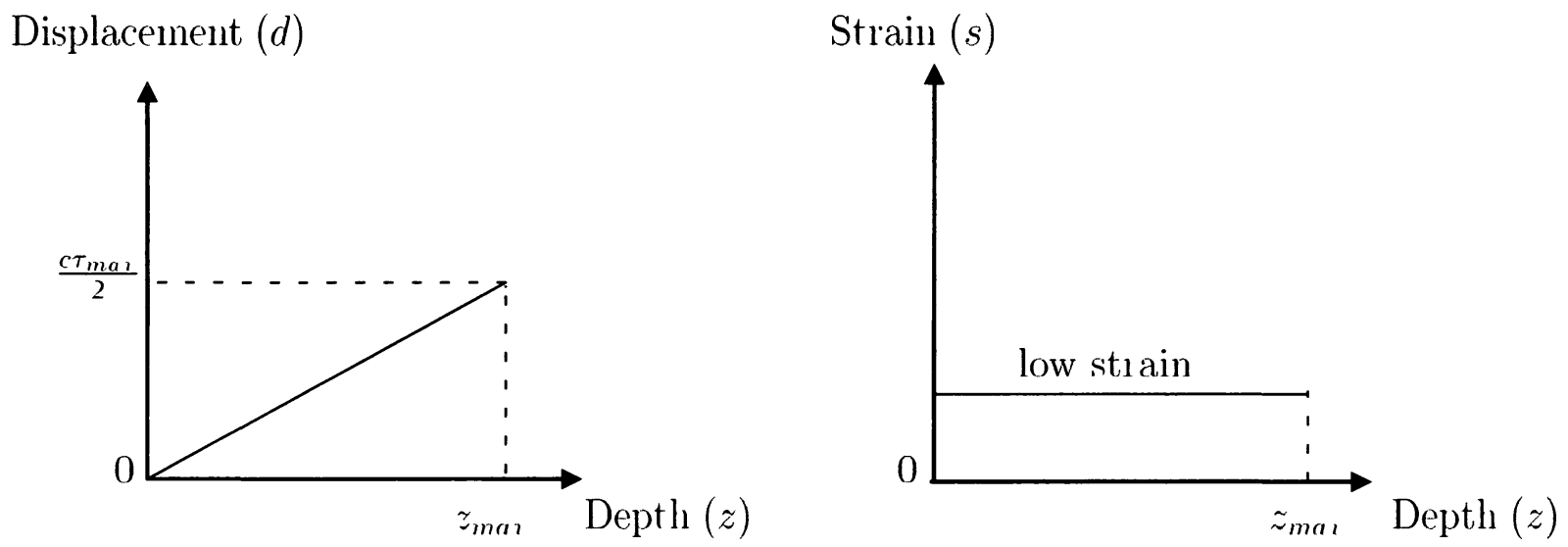

Figure 2.12 Curve of the local displacements of the particles with respect to their position (depth) in a compressed injured muscle (left) and corresponding strain curve (right).

\subsubsection{Transient Elastogrsaphy}

For the last 10 years, a number of methods have been proposed to estimate the viscoelastic properties of soft tissues by means of low-frequency (LF) shear wave propagation. By using LF vibration, quantitative values of shear elasticity and viscosity can be obtained and relative stiffness images can be made (Catheline, Wu, \& Fink, 1999). Transient elastography is a technique which functions in this way.

In this technique, a low-frequency transient (pulsed) vibration $(\sim 10-500 \mathrm{~Hz})$ is applied to produce displacements in tissue, which are then detected by pulse-echo ultrasound. On one side of the tissue, a small circular piston is positioned on the surface (on a vibrator) to generate vibrations and on the opposite side, the ultrasonic transducer is placed, with a common axis with 
the vibrator, to receive and represent echoes. In this technique, just tissue motions (displacements) caused by the shear wave propagation are selected and the numerical values of elasticity and viscosity are inferred from them. These numerical values are in fact, calculated based on the phase velocity of the shear waves $(\sim 5 \mathrm{~m} / \mathrm{s})$. At the end, relative stiffness image (slowness or inverse velocity image) of a tissue with different elasticities is made. Different shear wave velocities in different media result in an image with different slowness lines. In the image, the region where the velocity is lower (the soft medium) is bright, while the part where the velocity is larger (the hard or stiffer medium) is dark (Catheline, 1999; Taylor, 1999).

In the next section vibration sonoelastography, another type of ultrasound-based elastography, is explained. Before explaining this technique, it is worth to describe the Doppler Effect.

The Doppler Effect is the change in the frequency of sound caused by the relative motion of the source (ultrasound transducer) and target (tissue). Due to the relative velocities of the source and target, each received pulse is expanded or contracted having a different frequency with respect to the transmitted pulse. In medical ultrasound, the Doppler Effect is noticed in pulseecho mode where the transducer acts as both the source of the sound and the receiver of the Doppler-shifted echoes reflecting back from the tissue. This is normally done by a transducer with two separate crystals, each performing one of these functions. Similarly, the target (tissue) acts as both a moving receiver and a moving source which generates echoes returning to the transducer. Therefore, the echo received by transducer will be shifted as a result of both a moving receiver and a moving source. Doppler frequency obtained in this mode is twice the Doppler frequency obtained in either case alone and is shown by

$$
f_{d}=\frac{2 v \cos (\theta)}{c} f_{c}
$$


where $f_{c}$ is the source (transducer) frequency, $c$ is the acoustic velocity, $v$ is the velocity of moving object, and $\theta$ is the angle between direction of target motion and ultrasonic beam direction (Prince \& Links, 2006).

\subsubsection{Vibration Sonoelastography}

Sonoelastography is a rapidly developing ultrasound imaging technique that is mostly used for visualizing hard tumors in tissues. In this technique, a colour flow Doppler system along with external mechanical harmonic excitations (instead of acoustic impulse excitations) is applied to represent the elastic properties of tissue (Parker, Gao, Alam, Rubens, \& Lerner, 1996). A lowamplitude, low-frequency vibration (less than $0.1 \mathrm{~mm}$ displacement and around $100 \mathrm{~Hz}$ ) is externally applied to excite internal vibrations within the tissue. Then, in order to image the resulting vibration pattern, real-time Doppler techniques are utilized. A stiff tissue, such as a hard tumor or an injured muscle, beside the softer tissues (e.g., normal tissues) disturbs the normal vibration eigenmode (one of the normal vibrational modes of an oscillating system) pattern causing its amplitude to be reduced. This forms the basis for stiffness detection using sonoelastography (Gao, 1996; Taylor, 1999; Parker, 1996).

Normally, an audio speaker or a piston shaker is used as the external source to provide the low-frequency vibration. Doppler techniques are applied to estimate the vibration amplitude within a tissue under inspection. In gray scale images obtained by this technique, high amplitude vibration, corresponding to the softer tissue are bright and lower amplitude vibration, resulting from stiffer tissue is dark (Taylor, 1999; Parker, 1996). 


\subsection{Focus and Summary of the Thesis}

As explained in this chapter, information about stiffness (strain) of a tissue can be obtained with elastography using different imaging techniques. In clinical applications, the most commonly used imaging technique is an ultrasound. Among ultrasound-based elastography techniques, ultrasound compression elastography is chosen in this work. In this technique, a tissue is compressed while several RF signals are generated. Each of these RF signals becomes shorter (in time or depth) compare to the previously captured one due to time (phase) shifts occurred between them. Normally, resulted RF signals are considered as pairs and called RF signal pairs, where the first signal in each pair is called pre-compression RF signal and the second one is called the post-compression RF signal. This thesis aims to accurately measure the created phase shift between ultrasound pre- and post-compression RF signals during the compression process. It is this phase shift which is of interest and can be further used to provide displacement and strain information of the tissue. In the next chapters, the method by which the phase shift is measured will be explained. Then, different conditions and parameters that can affect this method causing the phase outcomes to be less accurate will be outlined. At the end, a novel approach for ultrasound phase reconstruction will be proposed by which the noise and unexpected fluctuations can be removed. It will be shown that this approach can act as a filter. 


\section{Chapter 3}

\section{Review of Data Acquisition Method}

The following chapter addresses the ultrasound imaging system used in the research done by Jason Silver in Department of Systems and Computer Engineering at Carleton University (Silver, 2009). It provides a description of the system itself, the ultrasound signals that the system is capable of emitting, and the real data measurements used throughout the research. This thesis uses measurements made by Jason Silver using his experimental setup.

\subsection{Ultrasound Imaging System}

In the research run by Silver, a medical ultrasound imaging system (Model: Picus, ESAOTE Europe, Maastricht, Netherlands) was used, which was accompanied by an Asus digital signal processing computer running Windows XP. The computer ran ART.LAB, the software that was used to both view and store data. The Picus machine used is shown in Figure 3.1.

\subsubsection{Ultrasound Probe}

The ultrasound probe applied includes a $40 \mathrm{~mm}$ linear array of transducers (Model: L10-5). The probe is contains four major layers and includes 127 individual transducers. The first layer contains the transducers made-up of piezoelectric crystals. The next two layers are impedance matching layers aimed to optimize the ultrasound waves' transmission into and out of biological tissue. The last layer is an acoustic lens utilized to focus ultrasound waves into the medium under inspection. 


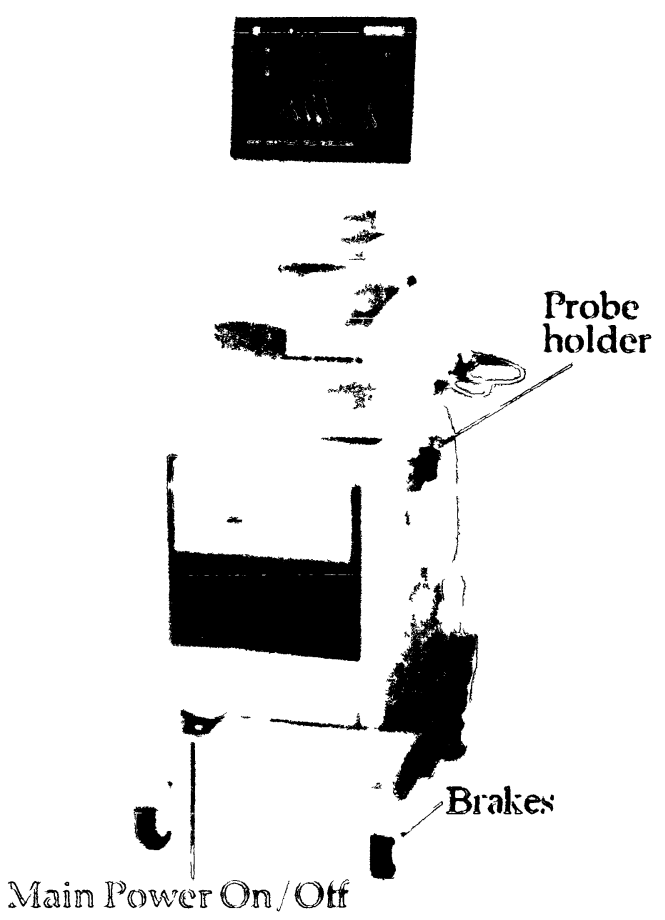

Figure 3.1 Picus ultrasound hardware. Reproduced from (Silver, 2009)

\subsection{Ultrasound Signal Specifications and Operation}

The ultrasound system samples the received signal every $0.03 \mu \mathrm{s}$. The center frequency of the transmitted ultrasound signals varied from 2.7-12.5 MHz. The system comprises of three major imaging modalities: M-mode, B-mode and Fast-B mode. The number of scan lines applied differs based on the acquisition mode. An M-mode obtains only a single line from a single location. Therefore, an M-mode frame has a single scan line. A B-mode frame obtains 127 scan lines from all 127 transducers in the linear array probe. Lastly, a Fast-B mode frame obtains 14 scan lines. 
The pulse repetition frequency (PRF) differs according to the imaging mode and is analogous to the frame rate. The frame rate is equal to the repetition rate of a signal from a single specific transducer identified as a line of sight. As a result, a frame is defined as follows: M-mode frames contain one scan line, Fast-B mode frames contain 14 scan lines and B-mode frames contain 127 scan lines.

The maximum frame rate in M-mode operation is $980 \mathrm{~Hz}$. A frame rate of up to $30 \mathrm{~Hz}$ is reachable in B-mode and the maximum frame rate of Fast-B mode is $687 \mathrm{~Hz}$. In M-mode, a single scan line over time is captured. In B-mode, for every temporal sample 127 scan lines are achieved. In B-mode and M-mode, axial signal acquisition is feasible for an overall of about 35 $m m$ in depth while in Fast-B mode $30 \mathrm{~mm}$ in depth is acquired. These values can change based on the acoustic velocity in the material(s) being measured. Based on the signal acquisition of the system, 5.66 seconds of data can be continuously captured.

In this thesis, the focus is on the M-mode imaging and the simulation (explained in chapter 4) is done based on this acquisition mode.

\subsection{Software and Data Format}

The ultrasound system used permitted to have access to any recorded digitized ultrasound RF signal. ART.LAB used to store data in a ".zrf" file format. This format was created by Esaote and permitted for signal analysis with Matlab by a function called "readzrf.m" which was included with the ultrasound hardware. The ART.LAB software, ".zrf" file format and "readZRF.m" function are described in the following. 


\subsubsection{Arterial Analyzer (ART.LAB) Software}

All data were stored by ART.LAB system. The ART.LAB (arterial analyzer) offers a real-time software based echo system extension and is designed for easy and accurate measurement of blood vessel properties by means of ultrasound. Its signal processing is based on RF-signal processing algorithms. The basic ART.LAB signal processing object is an RF-matrix which consists of several RF-signals. During real-time acquisition, the RF-matrices are stored in a circular buffer in the internal memory of the ART.LAB processing unit. In circular buffer the data is stored in a file with a ".zrf" extension.

\subsubsection{Data File Format with “.zrt" File Extension}

The data file with ".zrf" extension is formatted as follows: File header, Signal processing settings, Login information, Z-data matrix, RF-data matrix and Checksum. In file header, data about the file type, version, trigger and size are included. The signal processing settings encompass data about the way of data collecting. Information about machine software settings, ROI and information about a variety of options unique to an ART.LAB application are contained in this section. Details of the patient the data were collected on such as name, weight, blood pressure, etc are all included in the Login information. The data in the file header, signal processing settings, and login information were automatically removed by a supplied Matlab function in Silver's research which is consider as a reference for this research. The Z-data and RF-data matrices were the storage areas for the recorded data. The RF-data matrix contained 1516 sample points indicating the depth sample points. The Z-data matrix contained 758 sample points and demonstrates the envelope of the RF ultrasound signal. The total number of points in the RF-data matrix is always equal to twice of that found in the Z-data matrix. Finally, the 
checksum contained error checking that was automatically handled by the supplied Matlab function.

As it is clear from the name of RF-data matrix, it consists of a number of rows and columns corresponding to the depth sample points and scan lines, respectively. While operating in Mmode, the number of sample points in depth and scan lines are 1516 and 5550, respectively. Rows and columns of this matrix are referred to the axial and temporal directions, respectively.

\subsubsection{The readZRF.m Matlab Function}

The readZRF.m Matlab function is employed to read the ".zrf" data files. It takes the data file name (".zrf" file name), begin frame number and the number of frames as the input and gives the file information, Z-signal and RF-signal as the output.

\subsection{Data Processing Implemented by Silver}

After reading the data file by the readZRF function, the data file reprocessed by Silver for the purpose of his research. Although it was comfortable to have access to this digital information, this data was first preprocessed and then used for velocity and displacement estimations. For the preprocessing, he used specific methods to format the raw data properly and in turn, analyze the desired signals accurately. These methods are explained briefly in the following. More information about these methods is found in his thesis.

\subsubsection{Lens Echo Removal}

The existence of several layers between the transducer and the probe surface causes a certain time delay. This time delay results from ultrasound propagation inside the acoustic lens and the 
two impedance matching layers. By using the thickness of these materials and ultrasound propagation velocities in them, the time delay within each of these materials can be measured. By some calculation, this time delay (corresponding to 69 axial sample points within the probe) was removed from the beginning of every scan line during the preprocessing. Time delay removing was made to cancel out the lens echo (echoes generated from within the probe itself) and its corresponding time delay. Since lens echo were experienced by all received RF signals, the corresponding time delay periods were removed from RF signals before any data processing was performed.

\subsubsection{Temporal Reorganization}

Temporal reorganization addresses the way a file is stored during data acquisition. At any given time, the circular buffer contains about 6 seconds of data. As the storage option on the machine is selected and data are stored, the circular buffer is dumped precisely as it is into a file. This often results in a signal that is out of order with respect to time. It was therefore essential to correct this order during data preprocessing. This problem was fixed by locating the transition in the frame counter during the preprocessing. Consequently, the first scan line after the transition, represented the first recorded scan line at a reference time of $t_{0}$. All data were then reorganized in time using the determined $t_{0}$ as the reference starting time.

An example of the received RF- and Z-signal, obtained from one of the ".zrf" files via readZRF.m Matlab function and data reprocessing done by Silver, is shown in Figure 3.2 and Figure 3.3, respectively . 


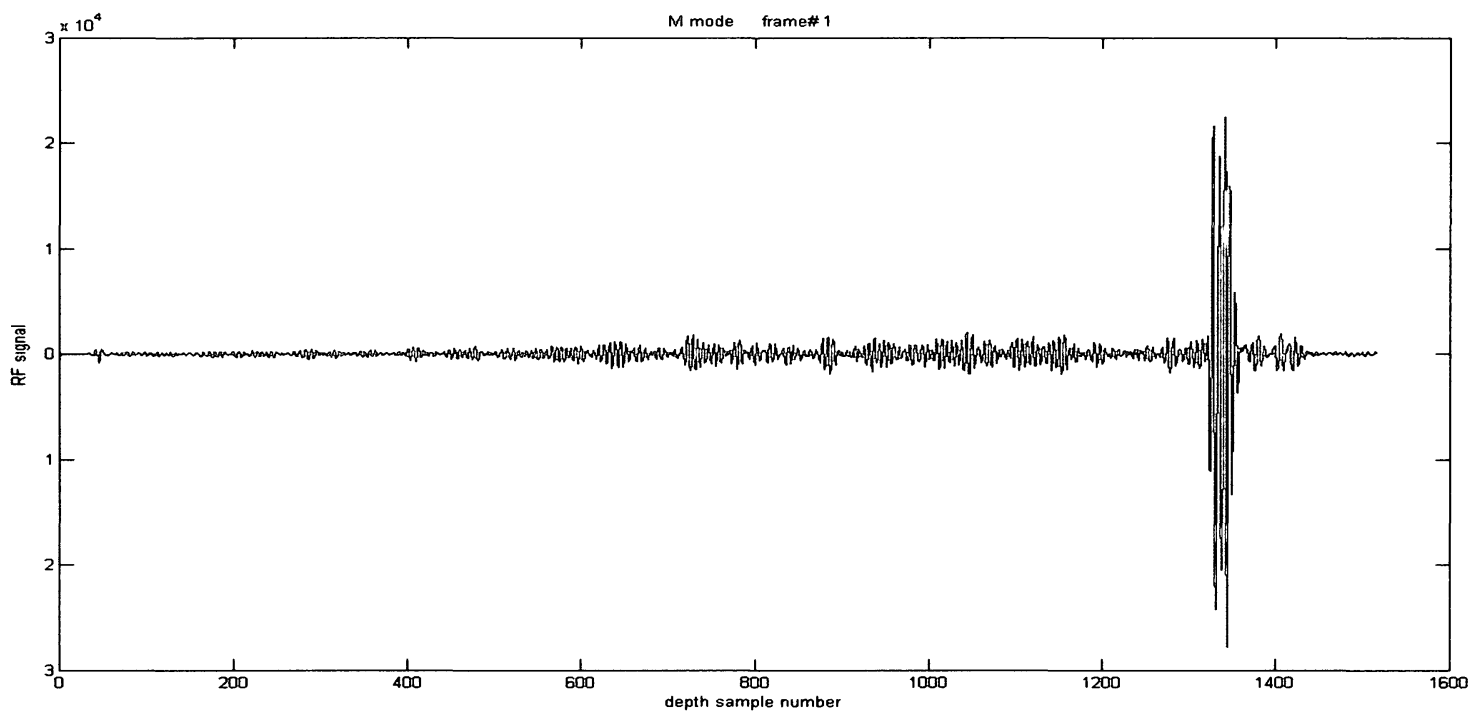

Figure 3.2 An example of a measured received RF signal with respect to the depth (sample number). M-mode is the acquisition mode; $\mathbb{R F}$ sigmal in the first frame is ploted in this fignre.

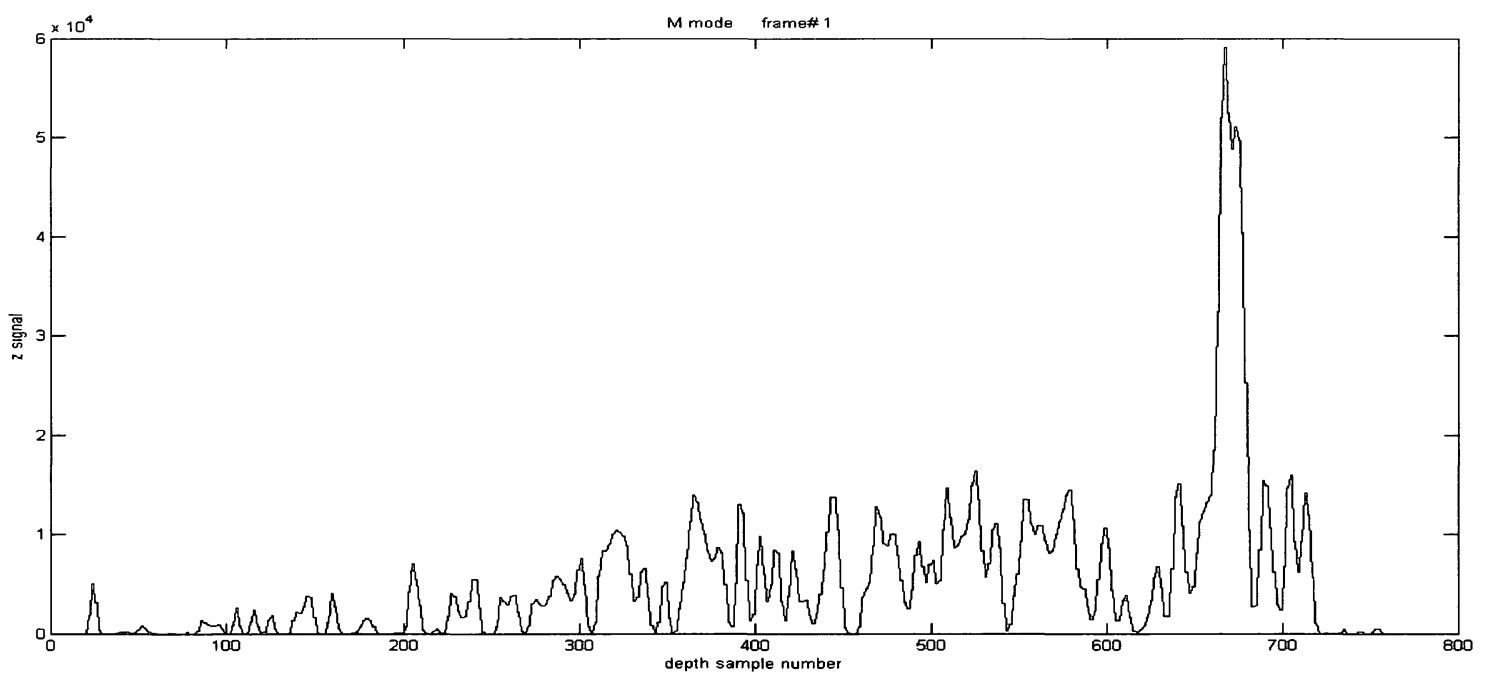

Figure 3.2 Corresponding measured Z-signal with respect to the depth sample number. M-mode is the acquisition mode; the figure shows the Z-signal obtained in the first frame.

\subsection{Data Files (“.zrf” files) and Experimental Testing}

In this research, some of the measured data stored in the ".zrf" files are used for the purpose of experimental testing. These data (RF- and Z-signals) were obtained in previous works where the 
ultrasound probe was used as a tool to compress (or expand) particular ultrasound phantoms simulating the skeletal muscles. In the experiment, it was tried to simulate muscle contraction. During phantom compression (or expansion), while the ultrasound system was in M-mode operation, several RF-signals (5550) were captured and stored. Since these RF signals were captured during the force application (probe motion), they are called pre- and post-compression (or expansion) RF signals. In this way, $5549 \mathrm{RF}$ signal pairs were created, each of which was made up of a pre- and post-compression (or expansion) RF signals. More explanation about measured data will be provided in chapter 7 where these data measurements are used for experimental testing.

In the next chapter, in a model-based computer simulation, pre- and post-compression RF signals will be simulated and a full discussion will be provided about the phase shift occurred between them. 


\section{Chapter 4}

\section{Simulation of Ultrasound Speckle echoes}

This chapter outlines the imaging environment, fast and slow time conventions, and computer simulation. It provides a description of the pulse-echo model and details the quadrature phase detection technique and its contribution to displacement estimation. At the end, the chapter defines the phasor method which will be used as a reference method to test the quadrature phase detection technique in different conditions.

\subsection{Imaging Environment}

Usually, a part of body which is being imaged made up of different types of tissues. It is presumed that this part of body contains a layer of soft material like fat. This layer is followed by one or more deeper layers of stiffer material such as muscle. The stiffer material is then followed by a layer of uncompressible material such as bone. A typical area which is under examination is shown in the Figure 4.1.

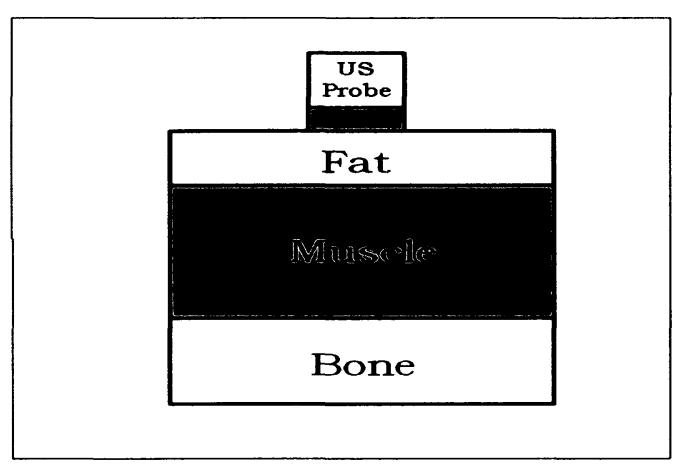

Figure 4.1 A typical part of body under examination; as it is shown, the order of different tissue layers are from the softer to the harder. 
The simulation used throughout this thesis relate to a specific imaging environment described in terms of a coordinate system. The coordinate system defines all amounts of compression/expansion measured with the ultrasound system to be relative to the ultrasonic probe surface. The frame of reference is then referred to as the ultrasonic probe reference frame and the amount of compression/expansion observed according to this coordinate system differs from what an experimenter would observe with a global frame of reference. Figure 4.2 and Figure 4.3 below represents the differences between identical probe motion while observed with respect to the two differing global and ultrasound probe frames of reference (Silver, 2007).

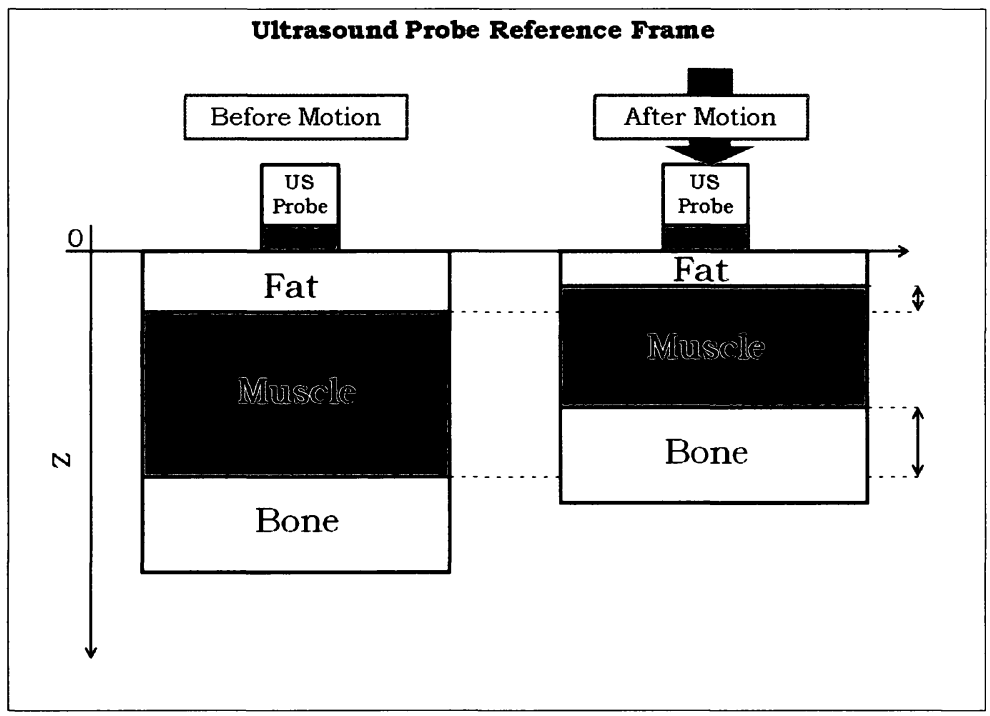

Figure 4.2 Probe motion as observed with respect to the ultrasound probe reference frame. The arrows show the amount of displacement due to the probe motion (Silver, 2009). 


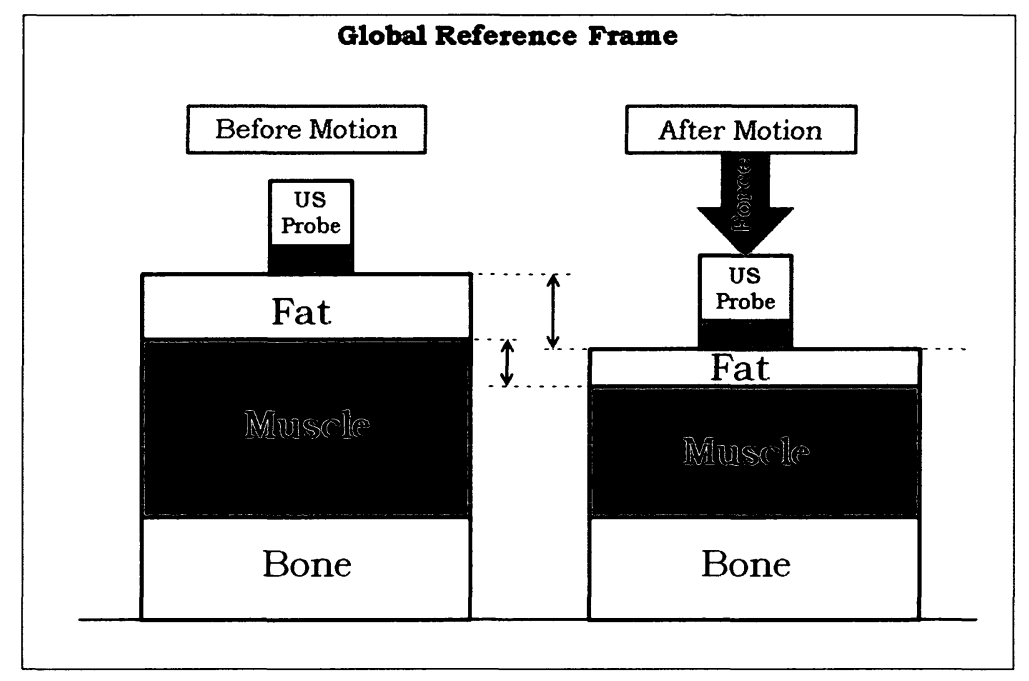

Figure 4.3 Probe motion as observed with respect to the global reference frame (Silver, 2009).

The observed displacement will increase with respect to depth when the reference frame is being at the probe surface. Specifically, it was assumed that for any constant force applied externally to the probe surface, the magnitude of the displacement observed would increase with respect to the depth. The result of this definition is that, by compressing a part of body, the muscle tissue is displaced more than the fat layer since it is located at a deeper position. The bone, however, is not compressed at all although it is located at the deepest position. This is due to the huge stiffness of the bone which may be considered as an uncompressible material.

Positive and negative displacements are defined as expansion and compression of the tissue, respectively.

In this thesis, it is assumed that the motion occurs in a direction parallel to the ultrasonic beam in the axial direction. Generally, the sample volume under examination can be compressed - or elongated, depending on whether the force (stress) is compressive or tensile. In this thesis, the stress is assumed to be compressive and by applying the force, the downward compression is meant. Figure 4.2 properly addresses all the assumptions made in this thesis. 


\subsection{Fast and Slow Time Conventions}

After defining the frame of reference, some variables and conventions related to the reference frame were defined. In the beginning, a distinction should be made between the fast and slow time conventions. When examining the ultrasonic signals, all information is related to time. Time, however, is used to denote two different quantities regarding to the axial (depth) and temporal directions often referred to as fast and slow time, respectively. In this thesis, the fast time is shown by $t$ and the slow time is represented by $T$ (Yu, 2006; De Ana, 2005). Fast and slow time along with different sampling conventions of M-mode and B-mode, are shown in Figure 4.4 and Figure 4.5. Variables $m, n$ and $i$ shown in these Figures represent temporal, axial and lateral samples, respectively. Fast time relates to the depth or axial samples of a received ultrasound signal while slow time refers to the time between transmitted pulses from a transducer. The latter is analogous to the pulse repetition frequency, frame rate or temporal sampling rate of the ultrasonic system based on the acquisition modes (Silver, 2007).

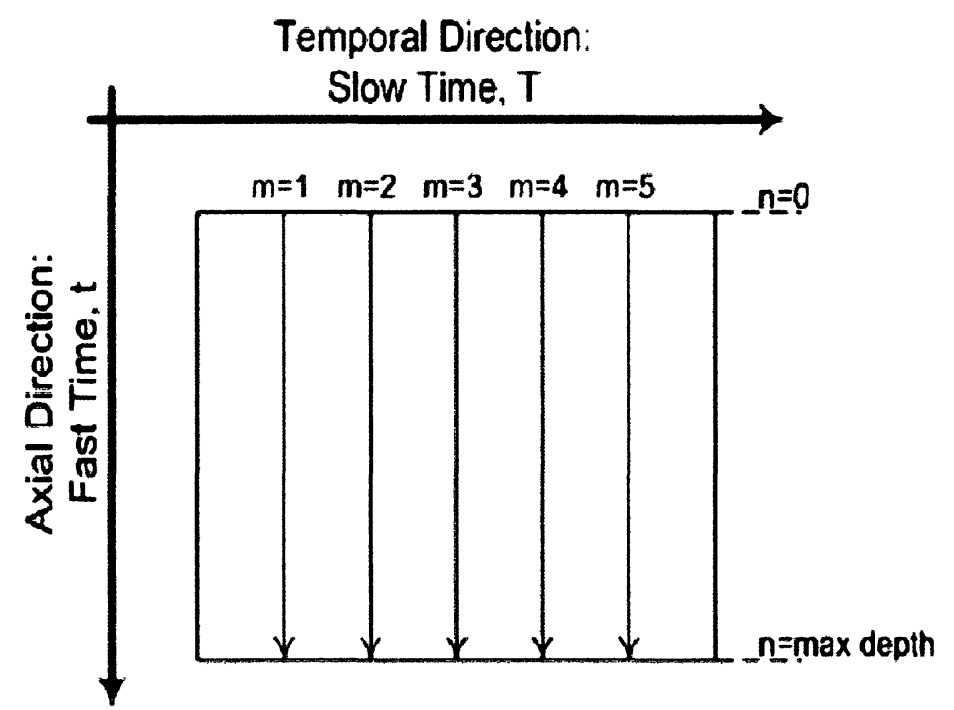

Figure 4.4 M-mode sampling structure. During M-mode, temporal samples, $\mathrm{m}$, are equivalent to scan lines and represent the pulse repetition frequency. Axial samples, n, represent depth. Reproduced from (Silver, 2007) 


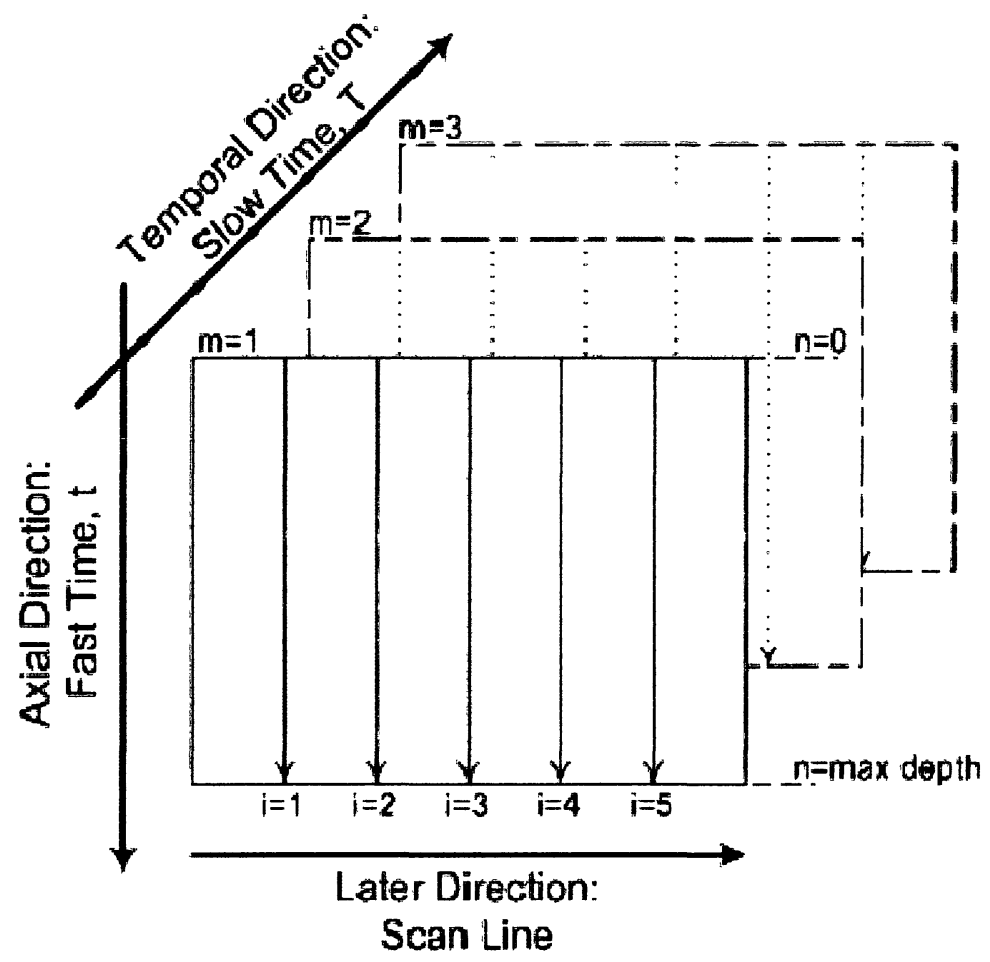

Figure 4.5 B-mode sampling structure; during B-mode, temporal samples, $\mathrm{m}$, are equivalent to image frame number. Scan line number, $i$, corresponds to samples in the lateral direction. Axial samples, $n$, represent depth. Reproduced from (Silver, 2007)

\subsection{Scatterers Contribution to Ultrasound Received RF Signal}

Different tissues of the body contain thousands of small particles and irregularities with different acoustic impedances. When an ultrasound beam is transmitted through the body, it encounters these particles locating in the beam propagation path, causing several echoes to be created at their position. As mentioned in chapter 2, these particles are referred to as scatterers and echoes resulting from them are often referred to as speckle echoes. The incident which strikes a single scatterer will be scattered many times due to collision with other scatterers in the way back toward the transducer. Among all scatterings, only those that travel in a similar path but in the opposite direction of the incident wave are detected by the ultrasound transducer. 
Speckle echoes contribute to creation of the received RF signals which are further used for doing measurements. In general, when a part of body with different constitutive layers is imaged, the received RF signal will be the superposition of all created speckle echoes plus the echoes created at the large boundaries where the type of tissue is changed. However, when just a certain layer such as the fat layer is examined, the RF signal will become the superposition of just speckle echoes. In this work, just one specific layer of a part of body is considered and thus, the RF signal is defined as the summation of speckle echoes. Figure 4.6. represents the contribution of scatteres to ultrasound received RF signal when just one layer is examined. In the computer simulation section, this contribution is shown mathematically.
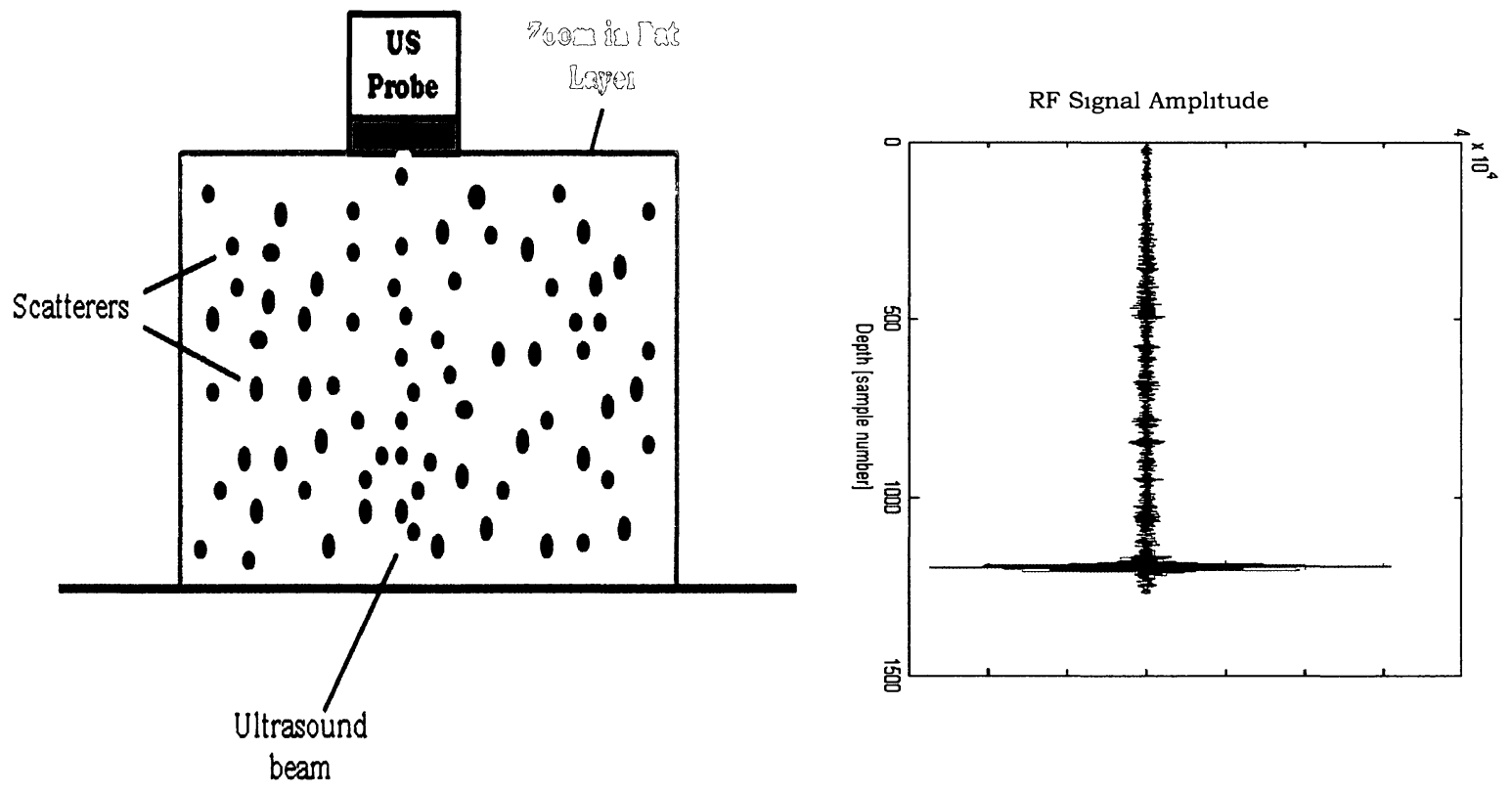

Figure 4.6 A microscopic version of a fat layer of a part of body with a number of scatterers distributed in it (left). These scatterers cause the echoes to be created at their positions. In the right plot, the received RF signal which is the sum of the created echoes is shown. It takes greater amplitude at the maximum depth (sample number) since at this depth the ultrasound signal encounters the bottom edge of the sample volume, which acts as a large and flat boundary. 


\subsection{Scatterers Contribution in a Deformed Medium}

Applying an external force causes the particles in a sample volume to be displaced toward or away from the probe. Based on our assumption, the particles are being compressed since the stress (force) is assumed to be compressive. Displacement of targets causes consecutive received echoes to experience a time delay with respect to the time of transmission. This time shift changes the phase relationship between pre- and post-compression received RF signals causing a phase shift to be created between them. It is this shift in phase that is of interest for our research. Since the phase shift of concern to our research is caused by the displacement of particles, measurements of phase shift can be obtained and converted to represent the amount of displacement and in return, the strain of the tissue under examination.

To create pre- and post- compression RF signals, an ultrasound probe is coupled to an object while transmitting an ultrasound signal through it. The received signal is called the precompression RF-signal which is shown by $\zeta(t)$. Then, the object is uniformly compressed (the probe is gradually pushed down toward the object) and another ultrasound signal is transmitted through it, resulting in the creation of second signal called the post-compression RF-signal, which is shown by $\zeta^{\prime}(t)$. In M-mode imaging done by the ultrasound system described in chapter 3, around $5550 \mathrm{RF}$-signals (5549 RF signal pairs) can be captured over 5.66 seconds. Each of these signals can be considered as a post-compression RF signal with respect to just previously captured one. That is, 5549 pairs of pre- and post-compression RF signals can be created in this mode.

Mathematical representation of ultrasound received RF signal and its constitutive echoes are described in the computer simulation section which is outlined next. 


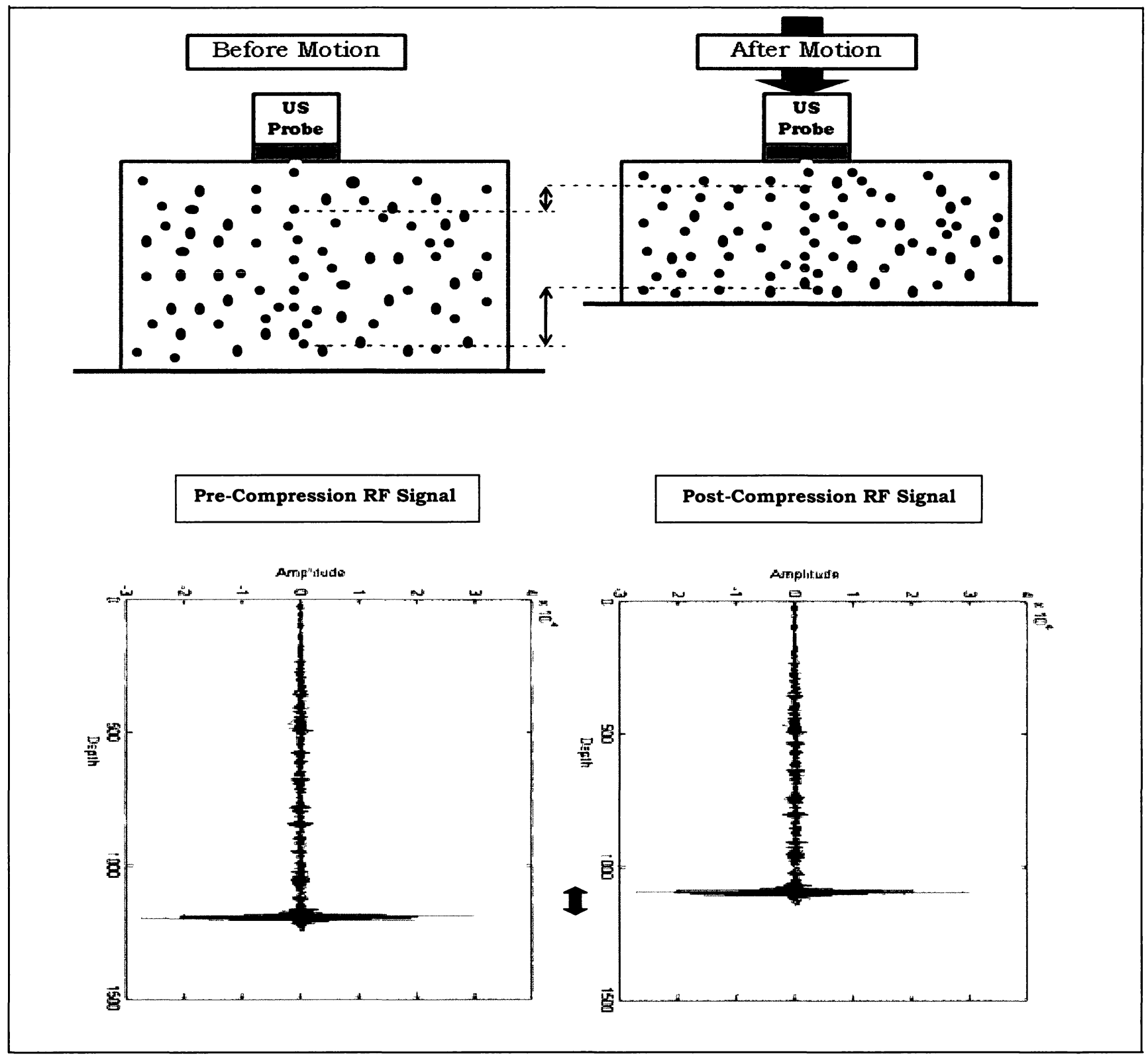

Figure 4.7 The sample volume before and after compression (top) and resulted pre- and post-compression RF signals (bottom). As it is shown, the post-compression RF signal is shorter than the pre-compression RF signal due to compression.

\subsection{Computer Simulation}

Computer simulation done in this research is written in Matlab programming. In this simulation, two general states of before and after compressing the sample volume (corresponding to the first 
two created RF-signals) are considered. The following section describes the pulse-echo model used in the simulation.

\subsubsection{Pre-Compression State $(\zeta(t))$}

\subsubsection{Gaussian Pulse-echo Model}

To simulate pulse and echo signals, the Gaussian pulse-echo model was used. Based on this model, the transmitted pulse is represented by

$$
P(t)=e^{-3(t)^{2}} \cdot \cos \left(\omega_{\mathrm{c}} t\right)
$$

where $t$ is the fast time or the time after pulse transmission, $\beta$ is the pulse bandwidth and $\omega_{c}$ is the ultrasonic center frequency. The transmitted signal encounters scatterers in its path causing echoes to be created at their positions. A single backscattered echo is represented by (Demirli, 2001; Qi, 2009).

$$
\begin{aligned}
S_{k}\left(\tau_{k}: t\right) & =\alpha_{k} P\left(t-\tau_{k}\right) \\
& =\alpha_{k} e^{-\beta\left(t-\tau_{h}\right)^{2}} \cdot \cos \left(\omega_{c} t-\omega_{c} \tau_{k}\right), \quad k=1: L
\end{aligned}
$$

where the notation $S_{k}\left(\tau_{k}: t\right)$ represents the $k^{t h}$ echo signal created. $t$ is the fast time and $\tau_{k}$ is the amount of change in the arrival time of the transmitted pulse which is determined based on the position of the scatterers. $\alpha_{k}$ is the cross section of the corresponding scatterer, $\beta$ is the bandwidth of echo, and the index $k$ refers to the $k^{\text {th }}$ echo. $L$ is the number of distributed scatterers in the sample volume, which is equal to 111 and 1055 for the two cases of having small and large amount of particles. These values were determined as small and large based on 
(Narayanan, Shankar, \& Reid, 1994). In this model, each echo is in fact, defined as the scaled and time delayed version of the transmitted pulse. Because of its Gaussian-shaped envelope, it is referred to as a Gaussian pulse-echo model (Demirli \& Saniie, 2001).

The echo model represented above has four parameters: $\alpha_{k}, \beta, \tau_{k}$. and $\omega_{c}$. Although the values of these parameters can vary from one echo to another, in this work it is assumed that $\alpha_{k}$ and $\tau_{k}$ are variables and other two parameters are constants. This assumption is made based on the degree of correlation between these parameters and the phase information of echoes. $\tau_{k}$ is one of the two parameters constituting the phase of echoes $\left(\omega_{c} \tau_{k}\right)$. It is translated to the phase and thus needed to be considered as a variable. Since it is directly related to the position of particles, which can be anywhere in the tissue range with the equal probability, it is assumed to be a uniform random variable. $\omega_{c}$ however, is assumed to be fixed to lessen the error in the phase estimation. This concept is further explained in the quadrature phase detection technique section in this chapter. Studying the effect of parameter $\beta$ on the phase of echoes is out of this work so it is also considered as a fixed value. In this work the bandwidth of all echoes are assumed to be the same, however, to test the functionality of the quadrature method in different conditions it is considered as a variable parameter changing from one $\mathrm{RF}$ signal to another. $\alpha_{k}$ is assumed to be a gamma-distributed random variable based on (Narayanan, Shankar, \& Reid, 1994). Its variability will not affect the phase and thus will not be considered as a source of error. From Eq. (4.2), time delay $\tau_{k}$. results in a change in the phase relationship between echo and transmitted pulse. The echo signal can be represented by

$$
S_{k}\left(\tau_{k}: t\right)=A_{k}(t) \cos \left(\omega_{c} t-\phi_{k}\right)
$$


with $A_{k}(t)=\alpha_{k} e^{-i\left(t-\tau_{k}\right)^{2}}$ and $\phi_{k}=\omega_{c} \tau_{k}$. Here, $A_{k}(t)$ and $\phi_{k}$ are the amplitude and phase information of the $k^{\text {th }}$ echo, respectively. The time delay $\tau_{k}$. is related to the position of the $k^{\text {th }}$ scatterer by Eq. (4.4) as (Hein \& O’Brien, 1993).

$$
\tau_{k}=\frac{2 x_{k}}{c}
$$

where $x_{k}$ is the position of the $h_{i}^{\text {th }}$ scatterer in the $z$-direction (depth of the tissue) and $c$ is the acoustic velocity in soft tissues $(\sim 1540 \mathrm{~m} / \mathrm{s})$.

\subsubsection{Received RF Signal Representation}

The received RF signal is defined as the superposition of all backscattered echo signals. Based on equations (4.1), (4.2), (4.3), and (4.4) it can be represented by (Molthen, 1998)

$$
S_{r}(t)=\sum_{k=1}^{L} S_{k}\left(\tau_{k}: t\right)=\sum_{k=1}^{L} \alpha_{k} P\left(t-\tau_{k}\right)=\sum_{k=1}^{L} \alpha_{k} P\left(t-\frac{2 x_{k}}{c}\right)
$$

where $P(t)$ is the transmitted signal, $S_{k}\left(\tau_{k}: t\right)$ is the $k^{\text {th }}$ echo signal $(1 \leq k \leq L)$, and $S_{r}(t)$ is the pre-compression received RF-signal. The block diagram of the creation of received RF-signal from the backscattered echoes is represented in Figure 4.8. 


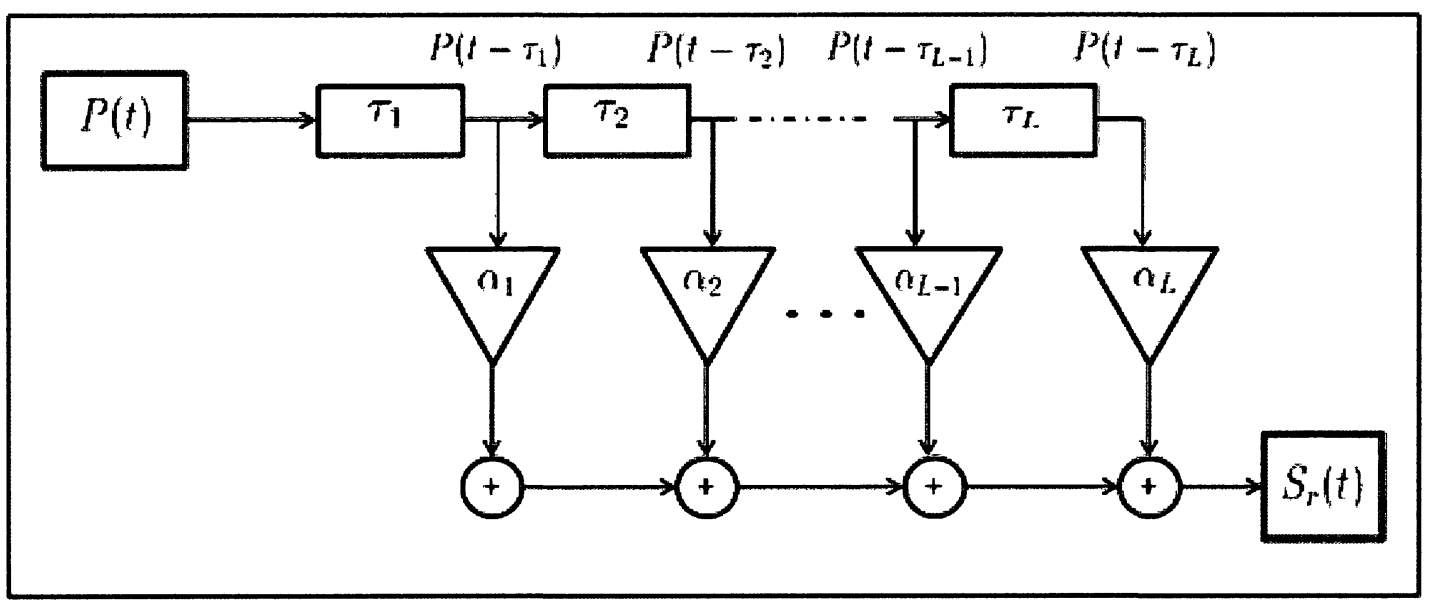

Figure 4.8 The block diagram of RF signal creation from the backscattered echoes. The transmitted signal is delayed and scaled at the position of each scatterer causing the corresponding echo to be created. Then, the sum of all these echoes will give the received $R F$ signal.

The received signal can also be represented by the amplitude and phase information of the echoes as (Shankar, 2000; Kolár̆ et al., 2005; Molthen, 1998)

$$
S_{r}(t)=\sum_{k=1}^{L} S_{k}\left(\tau_{h}: t\right)=\sum_{k=1}^{L} A_{k}(t) \cos \left(\omega_{c} t-\phi_{k}\right)
$$

In Eq. (4.6), the received RF signal is represented by sum of some sinusoids. The phase information of this RF signal is aimed to be calculated. In order to extract the phase information, one technique that can be applied is "quadrature phase detection" (Feng, 2006; Chang, 1993; Guirong, 1998; Molthen, 1998). In following sections, received RF signals and their constitutive echoes are represented in discrete forms which are the forms used in the simulation environment. 


\subsubsection{Quadrature Phase Detection Technique}

In practice, phase information of the received RF signal can be recovered by the quadrature phase detection technique. Since accurately recovering the phase information is the goal of this research, the quadrature technique is tested in different conditions throughout this research. Later on, certain parameters that affect this technique will be outlined. The following section details the quadrature detection method (Feng, 2006; Chang, 1993; Guirong, 1998; Molthen, 1998) which is applied to the discrete received RF signal represented by

$$
S_{r}(n)=\sum_{k=1}^{L} A_{k}(n) \cos \left(\omega_{c} n T_{s}-\phi_{k}\right)
$$

Here, $n$ and $T_{s}$ denote the axial depth sample number and axial sampling period, respectively.

During quadrature detection (also known as quadrature demodulation), received RF signal is multiplied by a reference sinusoid represented by

$$
S_{\text {ref }}(n)=e^{-\jmath \omega_{\text {dem }} n T_{s}}
$$

where $\omega_{\text {dem }}$ is the demodulation frequency. The quadrature detection process can then, be described by

$$
\begin{aligned}
I(n) & =L P F\left\{S_{r}(n) \cdot \cos \left(\omega_{d e m} n T_{s}\right)\right\} \\
& =L P F\left\{\left[\sum_{k=1}^{L} A_{k}(n) \cos \left(\omega_{c} n T_{s}-\phi_{k}\right)\right] \cos \left(\omega_{d e m} n T_{s}\right)\right\} \\
& =L P F\left\{\sum_{k=1}^{L}\left(\frac{A_{k}(n)}{2} \cos \left[\left(\omega_{c}-\omega_{d e m}\right) n T_{s}-\phi_{k}\right]+\frac{A_{k}(n)}{2} \cos \left[\left(\omega_{c}+\omega_{d e m}\right) n T_{s}-\phi_{k}\right]\right)\right\} \\
& =\sum_{k=1}^{L} \frac{A_{k}(n)}{2} \cos \left[\Delta \omega n T_{s}-\phi_{k}\right]
\end{aligned}
$$




$$
\begin{aligned}
Q(n) & =\operatorname{LPF}\left\{S_{r}(n) \cdot\left(-\sin \left(\omega_{\text {dem }} n T_{s}\right)\right)\right\} \\
& =\operatorname{LPF}\left\{\left[\sum_{k=1}^{L} A_{k}(n) \cos \left(\omega_{c} n T_{s}-\phi_{k}\right)\right]\left(-\sin \left(\omega_{d e m} n T_{s}\right)\right)\right\} \\
& =\operatorname{LPF}\left\{\sum_{k=1}^{L}\left(\frac{-A_{k}(n)}{2} \sin \left[\left(\omega_{c}+\omega_{d e m}\right) n T_{s}-\phi_{k}\right]+\frac{A_{k}(n)}{2} \sin \left[\left(\omega_{c}-\omega_{d e m}\right) n T_{s}-\phi_{k}\right]\right)\right\} \\
& =\sum_{k=1}^{L} \frac{A_{k}(n)}{2} \sin \left[\Delta \omega n T_{s}-\phi_{k}\right]
\end{aligned}
$$

where $I(n)$ and $Q(n)$, respectively, represent the in-phase and quadrature signals, LPF stands for low pass filtering and $\Delta \omega=\omega_{c}-\omega_{\text {dem }}$ is the down shift of the received ultrasound frequency. The filter used in this process is a lowpass prototype butterworth filter with the cutoff frequency of $1 \mathrm{MHz}$. Pre-compression complex baseband signal is represented by

$$
u(n)=I(n)+j Q(n)
$$

and the message or phase information of this baseband signal is obtained by

$$
\begin{aligned}
\angle u(n) & =\arctan \left(\frac{Q(n)}{I(n)}\right) \\
& =\arctan \left(\frac{\sum_{k=1}^{L} \frac{A_{k}(n)}{2} \sin \left[\Delta \omega n T_{s}-\phi_{k}\right]}{\sum_{k=1}^{L} \frac{A_{k}(n)}{2} \cos \left[\Delta \omega n T_{s}-\phi_{k}\right]}\right)
\end{aligned}
$$

where $\angle$ denotes phase angle. It can be seen from equation (4.12) that $\Delta \omega$ is a source of error in the phase estimation. Specifically, accurate phase estimation requires an accurate $\omega_{d e m}$ estimation such that it is as close to $\omega_{c}$ as possible. In this research it is assumed that $\omega_{\text {dem }}$ is equal to the $\omega_{c}$. As mentioned earlier, $\omega_{c}$ is assumed to be a constant parameter. If this 
assumption was not made the difference between $\omega_{c}$ and $\omega_{d c m}$ (down shift frequency of received RF signal) would become the source of error in the phase measurement process. By setting $\omega_{d c m}$ equal to the $\omega_{c}$ and canceling the coefficient of $1 / 2$ from the numerator and the denominator of Eq. (4.12), the phase of received RF signal will be simplified to

$$
\begin{aligned}
\angle u(n) & =\arctan \left(\frac{\sum_{k=1}^{L} A_{k}(n) \sin \left[-\phi_{k}\right]}{\sum_{k=1}^{L} A_{k}(n) \cos \left[-\phi_{k}\right]}\right) \\
& \equiv \varphi(n)
\end{aligned}
$$

where $\varphi(n)$ is the phase information of pre-compression received RF signal.

\subsubsection{Post-Compression State $(\zeta(t))$}

After compressing the sample volume, each particle is displaced to some extent, based on its position. Those particles located at deeper positions are displaced more than those in the shallower depths in the tissue. Subsequently, echoes will be created in the new positions and the superposition of these new echoes will give the post-compression received RF signal which can be represented by

$$
S_{r}^{\prime}(n)=\sum_{k=1}^{L} A_{k}^{\prime}(n) \cos \left(\omega_{c} n T_{s}-\phi_{k}^{\prime}\right)
$$

where $A_{k}^{\prime}(n)$ and $\phi_{k}^{\prime}$ are the amplitude and phase information of the $k^{\text {th }}$ echo after compressing the volume. The procedure defined above will be repeated for post-compression RF signal resulting in the post-compression complex baseband signal which is shown by $u^{\prime}(n)$. Similarly, 
by taking the angle of this baseband signal, the phase information of post-compression RF signal will be obtained

$$
\begin{aligned}
\angle u^{\prime}(n) & =\arctan \left(\frac{\sum_{k=1}^{L} A_{k}^{\prime}(n) \sin \left[-\phi_{k}^{\prime}\right]}{\sum_{k=1}^{L} A_{k}^{\prime}(n) \cos \left[-\phi_{k}^{\prime}\right]}\right) \\
& \equiv \varphi^{\prime}(n)
\end{aligned}
$$

where $\varphi^{\prime}(n)$ is the phase information of the post-compression RF signal.

It should be noted that even though the amount of displacement of the particles in the postcompression state is determined based on their positions and thus, will increase with respect to the depth, defining an increasing relationship between the time (phase) shift of the echoes and depth is not completely right. Each echo is generated when corresponding scattering is detected by the ultrasound. The scattering might however, hit many particles in its way back and not reach to the transducer quickly. Nevertheless, since the probability of occurrence of this phenomenon for all the particles before and after compression is the same, in this research this consideration is not taken into account. It is assumed that scatterings in pre- and post-compression states are taken place similarly (due to the same probability) and does not cause time delays in echo detection. Therefore, the time (phase) shifts created between the echoes after compression will be considered as the result of compression and thus, can be modeled to increases with respect to the depth.

\subsection{Phase Shift}

The phase shift of interest in this research is defined as the difference between the phase information of pre- and post-compression RF signals as 


$$
\Delta \varphi(n)=\varphi(n)-\varphi^{\prime}(n)
$$

Eq. (4.16) shows the phase shift between the first two consecutive RF signals corresponding to the first two scan lines or frames. To obtain the phase shift between the first RF signal (first scan line) and the last RF signal (e.g. the $5550^{\text {th }}$ scan line in M-mode imaging), all phase shifts occurred between consecutive RF signals should be accumulated.

\subsection{Displacement Estimation}

Once the pre- and post-compression complex baseband signals have been obtained, an estimation of displacement of the particles in the tissue can be made. Normally, the autocorrelation method (Loupas et al., 1995; Torp, 1994; Shamdasani, 2004; Pinton, 2006; Hasegawa, 2008; Tatar, 2003 ) is used to estimate the amount of displacement. However, in this research the phase shift resulted from subtraction is used. In the following subsections, it will be shown that these two ways give rise to the same results.

In order to exactly explain the procedure of displacement estimation, derivations are extended to include both the axial and temporal directions. In this way, ultrasound received RF signal can be represented by $S_{r z}(n, m)$, where $z$ is the ultrasonic beam direction, $n$ is the axial or depth sample number and $m$ is the pulse number of a transducer, scan line or frame number depending on the imaging mode. The complex baseband signal is represented by $u_{z}(n . m)$ and is summarized by

$$
u_{\tilde{z}}(n . m)=L P F\left\{S_{r z}(n, m) e^{-\jmath 2 \pi f_{d e m} n T_{s}}\right\}
$$

where $f_{d e m}=\frac{1}{2 \pi} \omega_{d e m}$. 


\subsubsection{Autocorrelation-based Quadrature Detection Technique}

As explained in chapter 2, time shifts occurred in RF signals as a result of compression can be estimated by cross correlation technique to be further used for local displacement estimation. Since the induced time shift results in the phase shift to be created between the RF signals, phase shift estimation can be done instead. A two-dimensional correlation function can be defined and the phase shift between RF signals can be calculated from it. The phase shift is then can be used for displacement estimation. The two-dimensional correlation function is defined as (Loupas et al., 1995; Torp, 1994; Shamdasani, 2004; Contreras, 2009; Hasegawa, 2008)

$$
r_{n . m}(\tilde{n}, \tilde{m}) \equiv u_{z}(n, m) \cdot u_{z}^{*}(n+\tilde{n} . m+\tilde{m})
$$

where $*$ represents the complex conjugate and $r_{n, m}(\tilde{n}, \tilde{m})$ defines the complex autocorrelation function with lags $\tilde{n}$ and $\tilde{m}$. in the axial and temporal directions, respectively. Displacement in the direction of ultrasound propagation can then, be calculated in the conventional phasesensitive method (Shamdasani \& Kim, 2004) using the estimated phase shift between two consecutive frames represented by

$$
\Delta \varphi_{z}(n, m)=\angle r_{n . m}(0.1)
$$

The phase shift shown in Eq. (4.19) is next used to estimate the displacement between two consecutive frames (RF signals) (Shamdasani et al., 2004; Hasegawa, 2008)

$$
\Delta d_{z}(n, m)=\frac{c \Delta \varphi_{z}(n . m)}{4 \pi f_{c}(n)} \cong \frac{c \Delta \varphi_{z}(n, m)}{4 \pi f_{c}}=\frac{c}{4 \pi f_{c}} \angle r_{n, m}(0,1)
$$


Estimation of phase shift between two consecutive frames by Eq. (4.19) is called "autocorrelation based quadrature detection technique".

\subsubsection{Phase Subtraction-based Quadrature Detection Technique}

In this thesis, instead of calculating the phase shift by autocorrelation $\left(\Delta \varphi_{:}(n . m)=\angle r_{n . m}(0.1)\right)$. the phase shift between two consecutive frames is simply calculated by subtracting the phase of pre- and post-compression RF signals, as described in Eq. (4.16). In this way, the phase shift between two consecutive frames is represented by

$$
\Delta \varphi_{z}(n . m)=\varphi_{z}(n . m+1)-\varphi_{z}(n, m)
$$

Displacement in the direction of ultrasound propagation is then, calculated in the same manner as follows

$$
\Delta d_{z}(n, m)=\frac{c \Delta \varphi_{z}(n, m)}{4 \pi f_{c}(n)} \cong \frac{c \Delta \varphi_{z}(n, m)}{4 \pi f_{c}}=\frac{c}{4 \pi f_{c}}\left(\varphi_{z}(n, m+1)-\varphi_{z}(n . m)\right)
$$

where $\Delta d_{z}(n . m)$ is the instantaneous displacement between two consecutive frames. Estimation of phase shift between two consecutive frames by Eq. (4.21) is called "phase subtraction based quadrature detection technique".

In Eq. (4.20) or (4.22), the center frequency varies with respect to depth (Loupas, Powers, \& Gill, 1995) and the knowledge of it is needed in order to obtain an accurate instantaneous displacement estimate. However, in this thesis, it is assumed that the center frequency is constant although ideally, it should be the actual center frequency $f_{c}(n)$ that is used. 
Afterwards, the accumulated displacement $d_{z}(n, m)$ is obtained by accumulating the estimated instantaneous displacement $\Delta d_{z}(n, m)$ between two consecutive frames (Hasegawa \& Kanai, 2008) as

$$
d_{z}(n . m+1)=d_{z}(n, m)+\Delta d_{z}(n . m) \quad \text { where } d_{z}(n, 0)=0
$$

The two ways of measuring phase shift, represented in Eq. (4.19) and (4.21), are similar in practice. This similarity is proved in the following section.

\subsubsection{Proof of the Equivalence}

In this section, autocorrelation based and phase subtraction based quadrature detection techniques are shown to be equivalent. As explained earlier, the in-phase and quadrature parts of the complex baseband signal are

$$
I(n)=\sum_{k=1}^{L} \frac{A_{k}(n)}{2} \cos \left(-\phi_{k}\right)
$$

and

$$
Q(n)=\sum_{k=1}^{L} \frac{A_{k}(n)}{2} \sin \left(-\phi_{k}\right)
$$

which constitute the complex baseband signal as follows 


$$
\begin{aligned}
u(n) & =I(n)+j Q(n) \\
& =\sum_{k=1}^{L} \frac{A_{k}(n)}{2} \cos \left(-\phi_{k}\right)+j \sum_{k=1}^{L} \frac{A_{k} \cdot(n)}{2} \sin \left(-\phi_{k}\right)
\end{aligned}
$$

For simplicity, we assume that the received signal is made up of only one echo. The proof can then, be generalized for the case of having several echoes. With this assumption, the complex baseband signal will become

$$
\begin{aligned}
u(n) & =\frac{A_{1}(n)}{2}\left[\cos \left(-\phi_{1}\right)+j \sin \left(-\phi_{1}\right)\right] \\
& =\frac{A_{1}(n)}{2} e^{-\jmath \phi_{1}}
\end{aligned}
$$

The two-dimensional correlation function with lag 0 and 1 in the axial and temporal directions, respectively, is defined as

$$
\begin{aligned}
r_{n . m}(0,1) & \equiv u_{z}(n, m) \cdot u_{z}^{*}(n . m+1) \\
& =\frac{A_{1}(n)}{2} e^{-\jmath \Phi_{1}} \cdot\left(\frac{A_{1}^{\prime}(n)}{2} e^{-\jmath \phi_{1}^{\prime}}\right)^{*} \\
& =\frac{A_{1}(n)}{2} e^{-\jmath \phi_{1}} \cdot \frac{1}{\frac{A_{1}^{\prime}(n)}{2} e^{-\jmath \phi_{1}^{\prime}}} \\
& =\frac{A_{1}(n)}{A_{1}^{\prime}(n)} \cdot e^{\jmath\left(\omega_{1}^{\prime}-\phi_{1}\right)}
\end{aligned}
$$

where $A_{1}(n)$ and $A_{1}^{\prime}(n)$ are the amplitude of the echo in pre- and post-compression states, respectively. Similarly, $\phi_{1}$ and $\phi_{1}^{\prime}$ are the phase of echo in pre- and post-compression states. 
Now, by taking the angle from both sides of Eq. (4.28), the following equivalence will be obtained.

$$
\angle r_{n . m}(0.1)=\phi_{1}^{\prime}-\phi_{1}
$$

Eq. (4.29) shows that the angle of the two-dimensional cross correlation function is equal to the phase subtraction. Since both ways of phase shift measurement lead to the same results, the phase subtraction method is chosen and used for the rest of this thesis.

As stated earlier, accurately recovering the phase information of received RF signals and in turn, accurately obtaining the phase shift created between them is the goal of this research. To do so, the quadrature detection technique is required to be tested in different conditions. In the following section, another approach, which we called it the "phasor method of phase measurement", is defined. This method is considered as a reference by which the quadrature detection technique is compared. Based on the amount of similarity between the results obtained from these two methods, the fidelity of the quadrature technique will be determined. Phase plots obtained by these two methods will be represented in chapter 5 .

\subsection{Phasor Method}

Based on pulse-echo model, the echo signal is defined as a sinusoid with the representation of

$$
S_{k}\left(\tau_{k}: n\right)=A_{k}(n) \cos \left(\omega_{c} n T_{s}-\phi_{k}\right) . \quad k=1: L
$$

The echo signals can also be represented by complex sinusoid as follows (from this onwards, the echo signal is represented by $\left.S_{k}(n)\right)$ 


$$
S_{k}(n)=\operatorname{Rcal}\left\{A_{k}(n) e^{j\left(\omega_{c} n T_{s}-o_{h}\right)}\right\}
$$

Based on the Euler's formula of $e^{\jmath \phi}=\cos (\phi)+j \sin (\phi)$. the complex sinusoid in Eq. (4.31) is equal to

$$
A_{k}(n) e^{\jmath\left(\omega_{c} n T_{s}-\phi_{k}\right)}=A_{k}(n)\left[\cos \left(\omega_{c} n T_{s}-\phi_{k}\right)+j \sin \left(\omega_{c} n T_{s}-\phi_{k}\right)\right]
$$

Comparing the two equations of (4.32) and (4.7), it can be seen that the real part in Eq. (4.32) is equal to the cosine term shown in Eq. (4.7).

In the complex sinusoid representation, if the phase portion is combined with the amplitude, the complex amplitude $X_{h}(n)$ will be obtained. That is,

$$
\begin{aligned}
A_{k}(n) e^{\jmath\left(\omega_{c} n T s-o_{k}\right)} & =\left(A_{k}(n) e^{\jmath\left(-o_{h}\right)}\right) e^{\jmath\left(\omega_{c} n T_{s}\right)} \\
& =\left(X_{k}(n)\right) e^{\jmath\left(\omega_{c} n T_{s}\right)}
\end{aligned}
$$

$X_{k}(n)=A_{k}(n) e^{\jmath\left(-\Phi_{h}\right)}$ is the complex amplitude which is also known as phasor (Dansereau, 2009). As mentioned earlier, the received RF signal can be represented by the sum of some sinusoids as

$$
S_{\imath}(n)=\sum_{k=1}^{L} A_{h}(n) \cos \left(\omega_{c} n T_{s}-\phi_{h}\right)
$$

Based on the above explanation, the received RF signal can also be represented by the complex sinusoids as 


$$
S_{r}(n)=\sum_{k=1}^{L} \operatorname{Re}\left\{A_{k}(n) e^{J\left(\omega_{c} n T_{s}-\phi_{k}\right)}\right\}
$$

This representation is used in the following theorem.

\section{Phasor Addition Theorem}

$L$ sinusoids with the same frequency $\omega_{c}$ can be summed up by adding their phasor parts (Dansereau, 2009):

$$
\begin{aligned}
\sum_{k=1}^{L} A_{k}(n) \cos \left(\omega_{c} n T_{s}-\phi_{k}\right) & =\sum_{k=1}^{L} \operatorname{Re}\left\{A_{k}(n) e^{J\left(\omega_{c} n T_{s}-\phi_{k}\right)}\right\} \\
& =\operatorname{Re}\left\{\sum_{k=1}^{L} A_{k} \cdot e^{J\left(-\phi_{h}\right)} e^{\jmath \omega_{c} t}\right\} \\
& =\operatorname{Re}\left\{\left(\sum_{k=1}^{L} A_{k}(n) e^{J\left(-\Phi_{k}\right)}\right) e^{\jmath \omega_{c} n T_{s}}\right\} \\
& =\operatorname{Re}\left\{\left(A(n) e^{\jmath(-\phi)}\right) e^{\jmath \omega_{c} n T_{s}}\right\} \\
& =A(n) \cos \left(\omega_{c} n T_{s}-\phi\right)
\end{aligned}
$$

where $A(n)$ and $\phi$ are the amplitude and phase of the signal resulted from adding the $L$ sinusoids. This summation can be shown by graphical vector addition. In Figure 4.9, it is shown for two complex sinusoids. 
Phasor Vectors

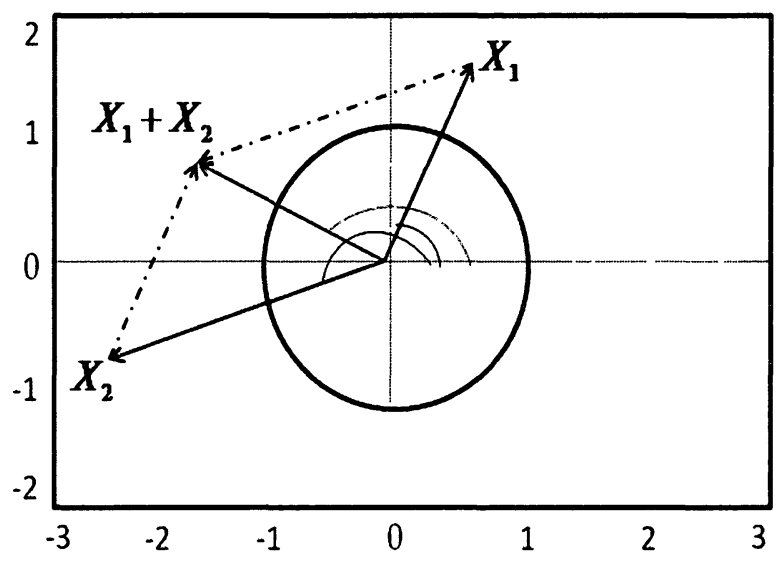

Figure 4.9 Summation of phasor parts of two complex sinusoids by graphical vector addition. The result of this summation is a vector with a new amplitude and phase information.

Since the received RF signal is the sum of echoes (sinusoids) with the same frequency of $\omega_{c}$ but different amplitude and phase information, phasor addition theorem can be applied to it. In the complex sinusoid representation of RF signal, the phase information can be obtained by taking the angle of the phasor part of the signal. Based on phasor addition theorem, RF signal's phasor part is resulted by adding up the phasor portions of constitutive echoes. Measuring the phase information of RF signal in this way is defined as phasor method. Although available in the simulation, the phasor approach cannot be applied to real ultrasound data (RF signals). Throughout this research, it is only used as a reference approach for testing the quadrature phase detection technique. As described, it can only be applied when the amplitude and phase information of echoes are known. This happens in the simulation where the values of these parameters are determined based on the relationships we defined. In reality, however, the only thing that can be measured is the received RF signal and the whole representation of it. 
Therefore, having access to the echoes' information is not possible and in turn, the phasor approach cannot be applied to measure the phase information.

As mentioned in the beginning of this chapter, local displacements of the sample volume under the compression increase with respect to the depth when the reference frame is being at the probe surface. These local displacements are directly related to the time (phase) shift created between received RF signals. One can say that a straight line which increases with respect to the depth can be considered as a reference model to which the quadrature phase shift results can be compared. This model however, was not considered as a reference in this thesis since the increasing trend of the resulted phase shift plots are not completely linear and slight fluctuations are appeared in them due to the particles' distribution. By the phasor method, it was possible to simulate those fluctuations. Therefore, the phasor method was considered as a reference method by which the quadrature technique can be compared. In chapter 5 it will be shown that the phasor method, like all other phase measurement methods, can cause $2 \pi$ phase jumps to be created in the phase shift results. Since these jumps can be fixed by unwrapping processes, this limitation of the phasor method was not taken into account.

This chapter closes with the explanation of value settings in the computer simulation. In the next section, details of the values used in simulation are outlined.

\subsection{Setting Values in Computer Simulation}

As explained in chapter 3, certain data were measured during ultrasound phantom examinations in previous works and given as a source in this research. These measured data were stored in the files with .zrf file extension. In an experiment, the ultrasound phantom was compressed due to the probe motion and certain data such as RF-data matrix, complex baseband signal, and the 
extracted phase information were measured. The measured RF-data matrix consists of a number of rows and columns which corresponds to the depth sample number and temporal sample number (scan lines), respectively. In M-mode operation, the depth sample number and scan lines were 1516 and 5550, respectively. The axial signal acquisition was feasible for $35 \mathrm{~mm}$ in depth. The center frequency $f_{c}$ and sampling period $T_{s}$ were set equal to $5 \mathrm{MHz}$ and $0.03 \mu s$, respectively. These values were defined based on the machine (system) properties and operation.

In order to have a realistic simulation, parameters defined in the simulation were set close to those of the measured data. Details of value setting are explained in the following. As it is stated earlier, the received RF signal has the continuous form of

$$
S_{r}(t)=\sum_{k=1}^{L} \alpha_{k} e^{-\beta\left(t-\tau_{k}\right)^{2}} \cdot \cos \left(2 \pi f_{c}\left(t-\tau_{k}\right)\right)
$$

and the discrete form of

$$
S_{r}(n)=\sum_{k=1}^{L} \alpha_{k} e^{-\beta\left(n T_{s}-n_{\tau_{k}} T_{s}\right)^{2}} \cdot \cos \left(2 \pi f_{c}\left(n T_{s}-n_{\tau_{k}} T_{s}\right)\right)
$$

where $T_{s}, n$, and $n_{\tau_{k}}$ are respectively, sampling period, depth sample number, and the amount of shift in the sample number corresponding to the time shift of $\tau_{k}$ in the discrete form representation of the RF signal. In the simulation, different parameters of the waveform represented in Eq. (4.38) were set as follows

- The center frequency $\left(f_{c}\right)$ of transmitted signal, was set to be $5 \mathrm{MHz}$. 
- Sampling period $\left(T_{\varsigma}\right)$ was assumed to be equal to $0.03 \mu s$ (samples were taken every 0.03 $\mu s)$.

- The cross section of the $k^{\text {th }}$ scattrere $\left(\alpha_{k}\right)$ was defined as a gamma-distributed random variable with scale 1 and shape 2 :

$$
\alpha_{k} \sim \operatorname{Gamma}(2,1)
$$

- Bandwidth of the measured RF signal was calculated based on $3 \mathrm{~dB}$ criterion (the distance between two points in the frequency domain where the signal is $\frac{1}{\sqrt{2}}$ of the maximum signal amplitude (half power)). It was found to be $0.8 \mathrm{MHz}$. As a result, the bandwidth of the model echo signals $(\beta)$ in the simulation was assumed to be equal to this value. In Figure 4.10, the amplitude spectrum of the measured RF signal is shown.

- Position of particles was defined as a uniform random variable between 0 and $33 \mathrm{~mm}$.

- Maximum depth and initial depth sample number $(n)$ were set to $35 \mathrm{~mm}$ and 1516 , respectively. Each time by obtaining a new post-compression RF signal, $n$ was reduced. In order to have the same-size vectors for pre- and post-compression RF signals (redundant), the post-compression RF signals were zero-padded.

- The number of scatterers $(L)$ was set to 117 and 1055 for the two cases of having few and large amount of particles based on (Narayanan, Shankar, \& Reid, 1994). In the referred article, for the range cell of $1.26 \mathrm{~mm}, L$ equal to 4 and 38 was considered as the small and large amount, respectively. Thus, for the tissue range of $35 \mathrm{~mm}$ (in this research), 111 and 1055 were used to represent the small and large values, respectively. 


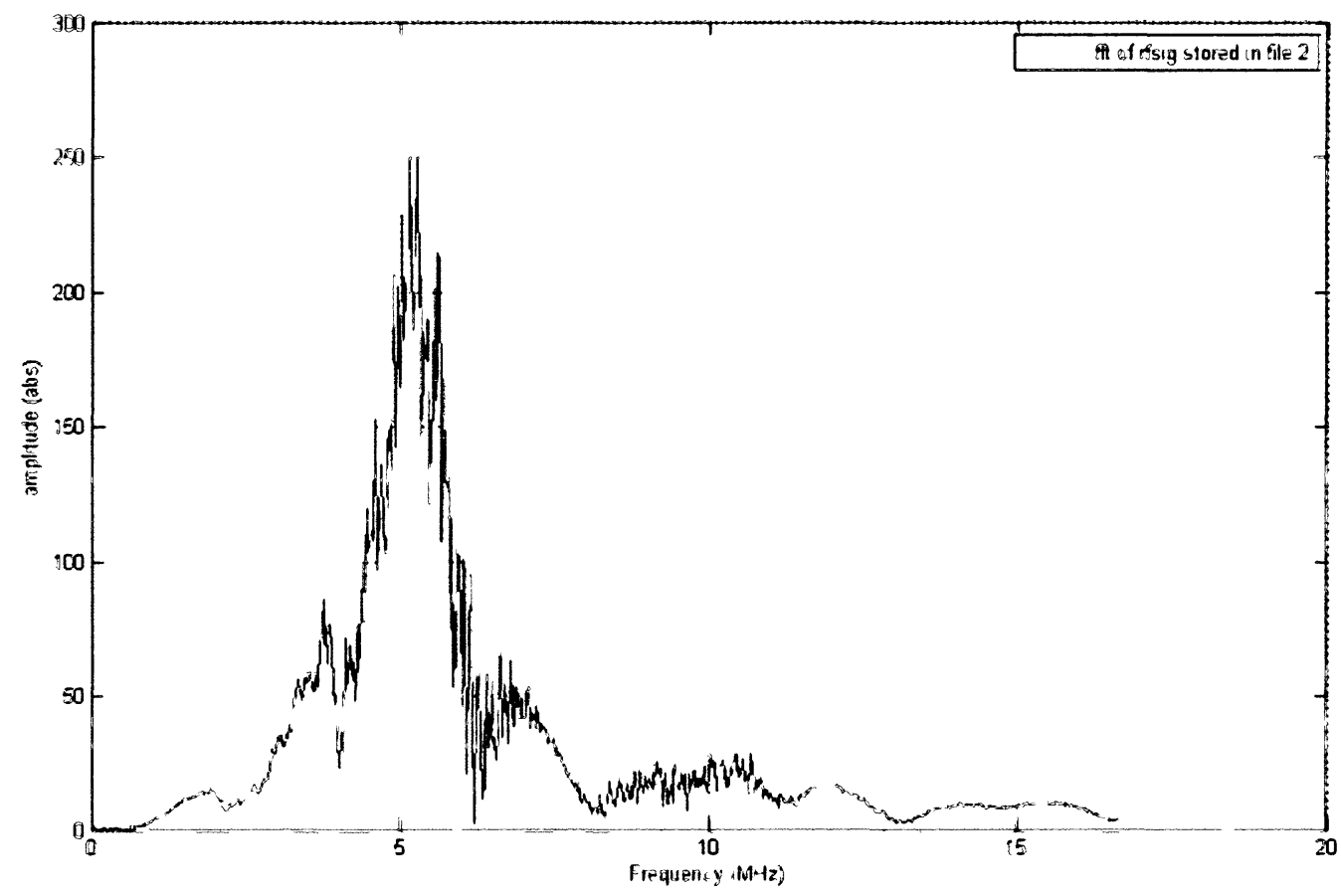

Figure 4.10 Amplitude spectrum of the measured data around a center frequency of $5 \mathrm{MHz}$. The signal from which the fft is taken is the RF signal stored in one of the files with .zrf extension. 


\section{Chapter 5}

\section{Numerical Simulation Results}

This chapter outlines several phase shift plots obtained from the simulated RF signal pairs. It describes the relationship between instantaneous displacements of the sample volume and the phase shifts created between consecutive received RF signals. It provides a discussion about phase jumps appeared in the phase shift plots. At the end, it closes with the examination of quadrature phase detection technique in different conditions.

\subsection{Instantaneous Displacement Effect on Consecutive Phase Shift}

As stated before, in M-mode operation only one RF signal is created in each frame. To create certain number of RF signals, the same number of frames is required. The simulation of the first two consecutive RF signals (frames) and the way of calculating the phase shift occurred between them was explained in chapter 4. In this chapter, generation of more RF signals, corresponding to the number of required frames, is considered.

In chapter 4, it was briefly explained that the phase shift occurred between consecutive frames and instantaneous displacement of the sample volume have the following relationship (Shamdasani, 2004; Hasegawa, 2008)

$$
\Delta d_{z}(n . m)=\frac{c}{4 \pi f_{c}}\left(\Delta \varphi_{z}(n, m)\right) . \quad n=1: N \cdot m=1: M
$$


where $\Delta d_{z}(n, m)$ is the instantaneous displacement of the volume at sample number $n$ and frame number $m$, and $\Delta \varphi_{z}(n, m)$ is the phase shift occurred between consecutive frames of $m$ and $m+1$, at sample number $n$.

It is also explained that, the accumulated displacement of the volume can be calculated by accumulating the estimated instantaneous displacements as (Hasegawa, 2008)

$$
d_{z}(n, M)=\sum_{m=1}^{M} \Delta d_{z}(n . m) . \quad n=1: N
$$

where $d_{z}(n . M)$ is the accumulated displacement of the volume at sample number $n$ while $M$ number of frames are generated.

In simulation, $\Delta d_{z}(n . m)$ was set to different values to see if it has any effects on the $\Delta \varphi_{i}(n . m)$ or not. This examination was done to find the optimal value(s) of instantaneous displacement(s) that would result in the expected consecutive phase shift result. The expected consecutive phase shift plot should increase with respect to the depth. Resulted optimal value(s) of $\Delta d_{z}(n, m)$ determines how slow ultrasound probe should be pushed down on the volume during the compression. In the following section, the effect of different values of $\Delta d_{z}(n, m)$ on the resulted $\Delta \varphi_{z}(n, m)$ is studied.

\subsubsection{Simulation Results}

It was assumed that the accumulated displacement at maximum depth sample number $N$ after creation of $M$ frames $\left(d_{z}(N . M)\right)$ is about $1 \mathrm{~mm}$ and the amount of displacement occurring between two consecutive frames of $m$ and $m+1$, at the maximum depth sample number 
$\left(\Delta d_{z}(N, m)\right)$ is $50 \mu m . \Delta d_{z}(N, m)$ is in fact the instantaneous displacement of the entire volume since the maximum depth sample number represents the whole depth or equivalently the entire volume. Once $d_{z}(N, M)$ and $\Delta d_{z}(N, m)$ were determined, the number of required frames was calculated by

$$
\text { Number of Frames }=\frac{d_{z}(n, M)}{\Delta d_{z}(n, m)}+1
$$

Figures 5.1 and 5.2 represent the first (pre-compression) and second (post-compression) simulated RF signals along with their phase information. Figure 5.3 shows the amount of phase shift occurred between these two consecutive RF signals. In Figure 5.3, the top plot is obtained via quadrature technique while the bottom plot is obtained by the phasor method. In all phase shift plots in this chapter, the top plot corresponds to the quadrature technique, while the bottom plot corresponds to the phasor (reference) method. 

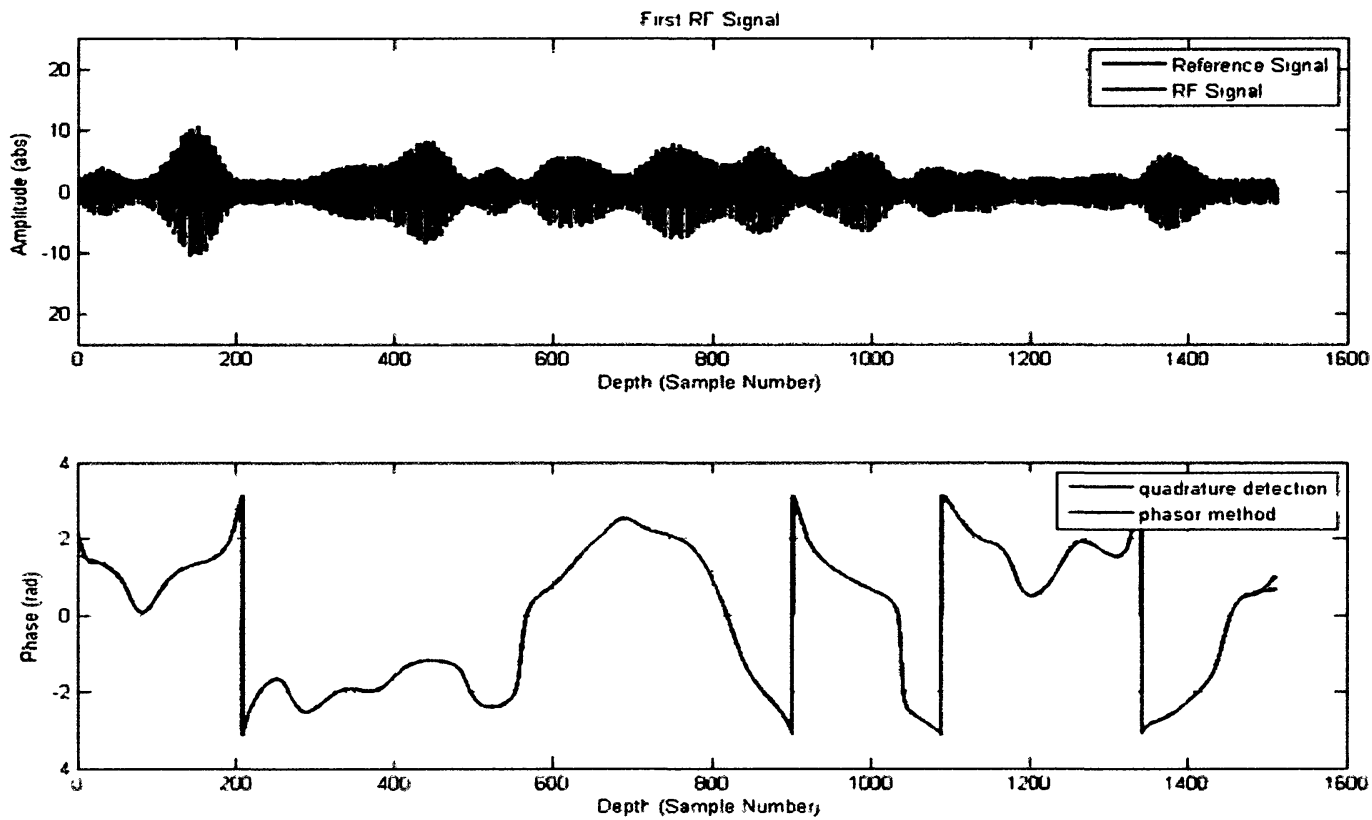

Figure 5.1 Pre-compression RF signal (top) and its phase information (bottom). In the bottom plot, the phase obtained by the quadrature method is shown in blue colour and the one obtained via phasor method is in green. Two plots are almost matched at this step.
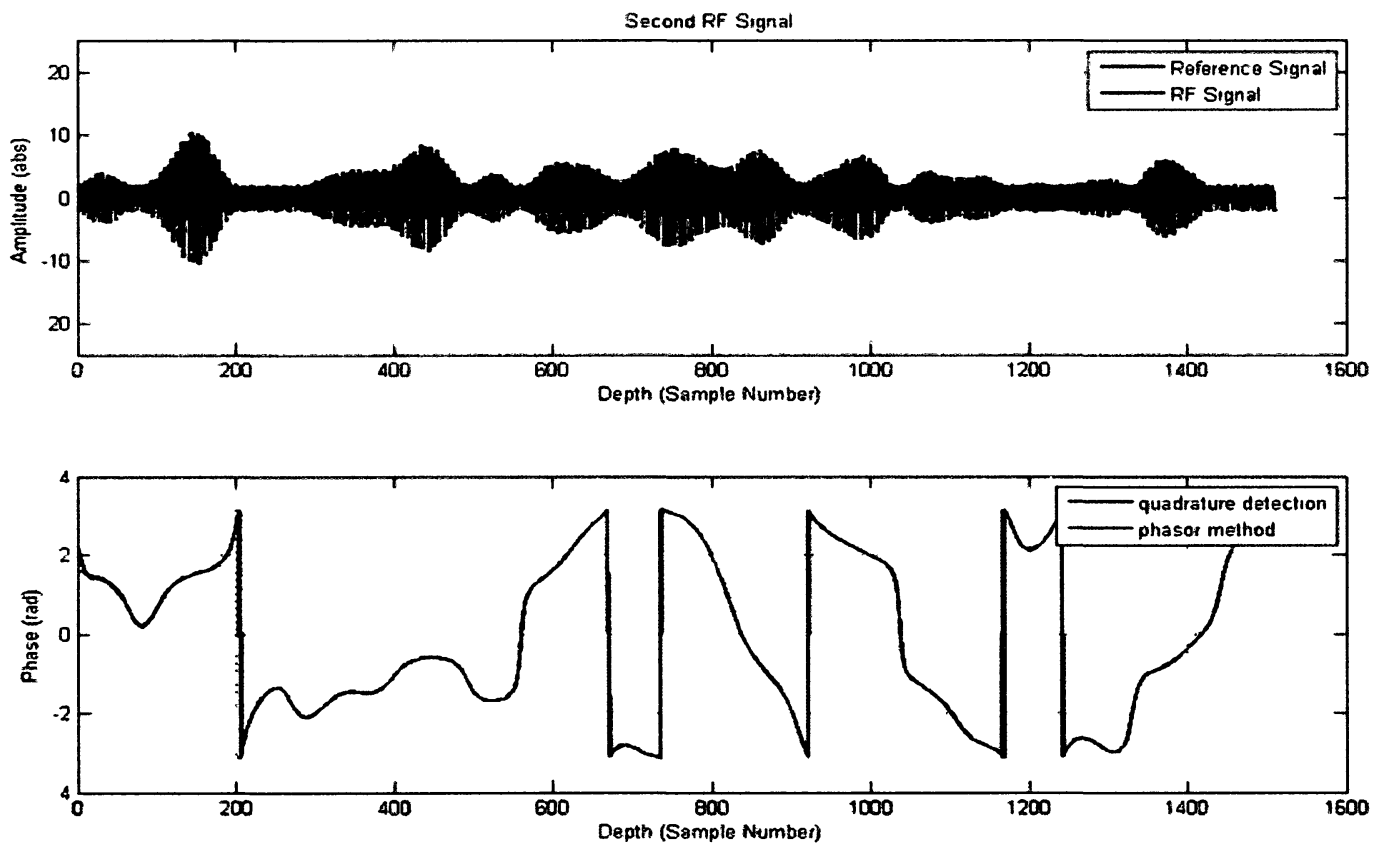

Figure 5.2 Post-compression RF signal (top) and its phase information (bottom). Two phase plots that are obtained by the quadrature and phasor methods are represented in blue and green colours, respectively. 

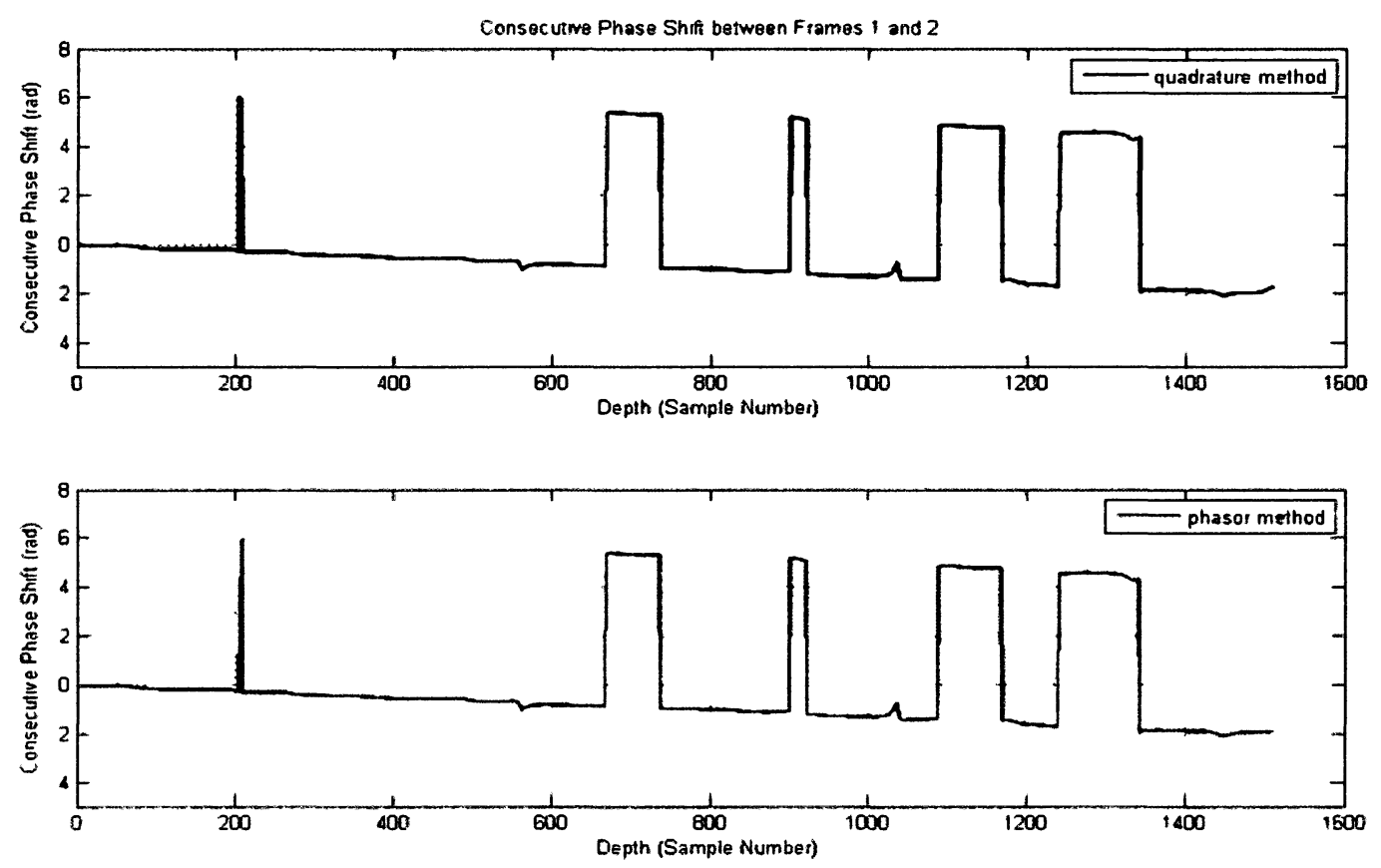

Figure 5.3 Created phase shift between pre- and post-compression RF signals. The resulted phase shift is equivalent to 50 $\mu \boldsymbol{m}$ displacement of the total sample volume after compression. The top plot is obtained via the quadrature method while the bottom one is obtained by the phasor (reference) method.

In Figures 5.1 and 5.2, phase plots obtained via quadrature technique match the results obtained by phasor method. This means that the quadrature technique is functioning well at this step. Later on in this chapter, different conditions causing two methods result in different outcomes will be explored.

In Figure 5.3, the magnitude of the phase shift observed increases with depth, as expected. A negative phase shift denotes a displacement in a direction towards the probe. The maximum phase shift is occurred at the maximum depth sample number of 1515 . At this sample number, the magnitude of the phase shift is about $2.0 \mathrm{rad}$ which is equivalent to the maximum displacement of $50 \mu \mathrm{m}$, based on Eq. (5.1). 
However, in this figure at certain depth sample numbers such as 200,680 , and 900 , phase shifts of about $2 \pi$ are created causing the plot not to be completely linear. To see if phase jump creation is related to the instantaneous displacement of the sample volume or not, different values of $\Delta d_{z}(N . m)$ were examined.

The examination was done for $\Delta d_{z}(N . m)$ equal to $0.1,1,5,10,20,50,100$, and $150 \mu \mathrm{m}$. Based on the assumption that the accumulated displacement of the entire volume $\left(d_{z}(N . M)\right)$ is $1 \mathrm{~mm}$. for each of these values the frame numbers was found to be 10001, 1001, 201, 101, 51, 21,11 , and 8 , respectively. As an example, the resulted phase shift plots for $\Delta d_{z}(N . m)$ equal to $0.1 \mu \mathrm{m}$ is shown in the following figures. In Figures 5.4 and 5.5, the consecutive phase shift $\left(\Delta \varphi_{z}(n . m)\right)$ between the first two RF signals and corresponding accumulated phase shift after 501 frames is shown, respectively.
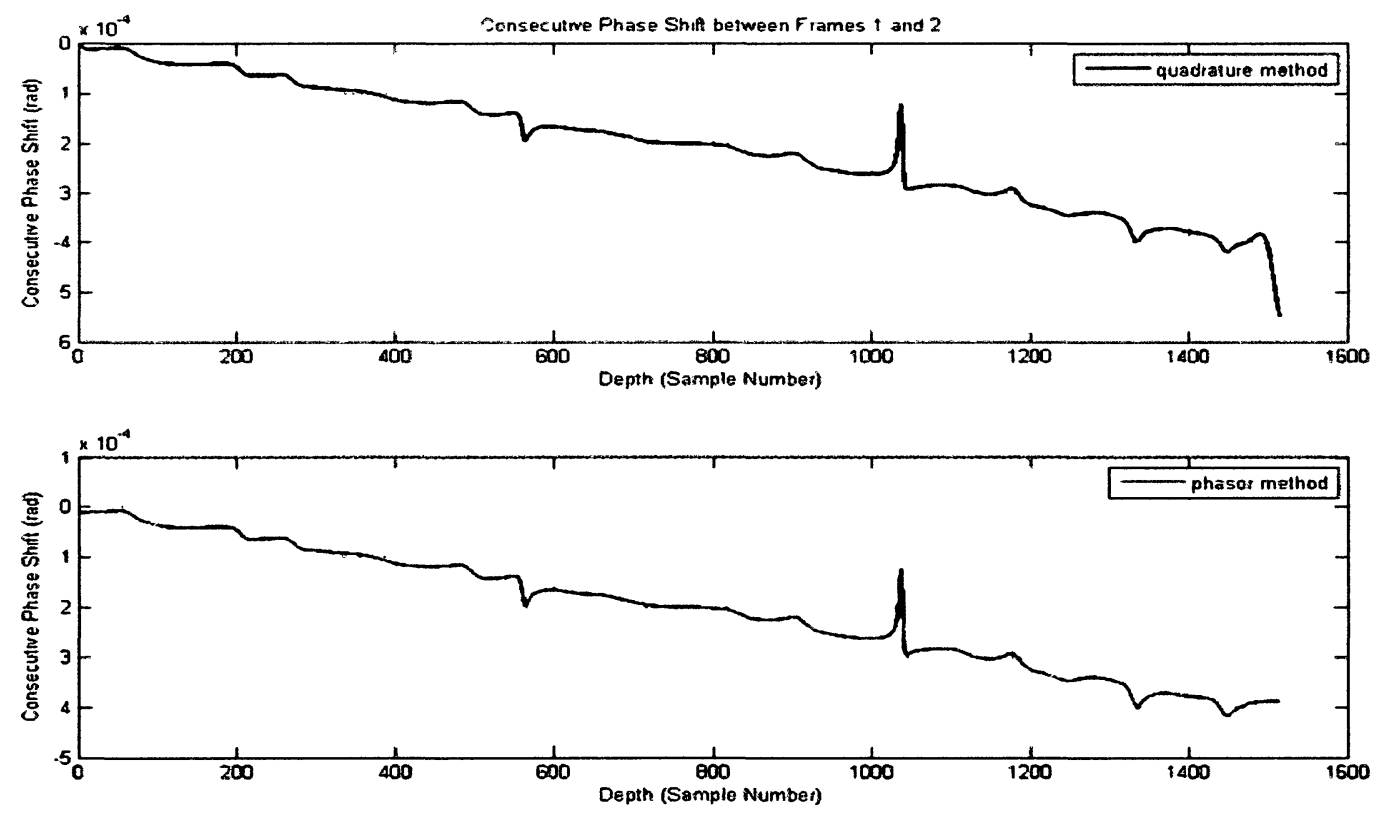

Figure 5.4 Phase shift between pre- and post-compression RF signals, equivalent to $0.1 \mu \mathrm{m}$ displacement of the total sample volume after compression. The top plot is obtained via the quadrature method while the bottom one is obtained by the phasor (reference) method. 

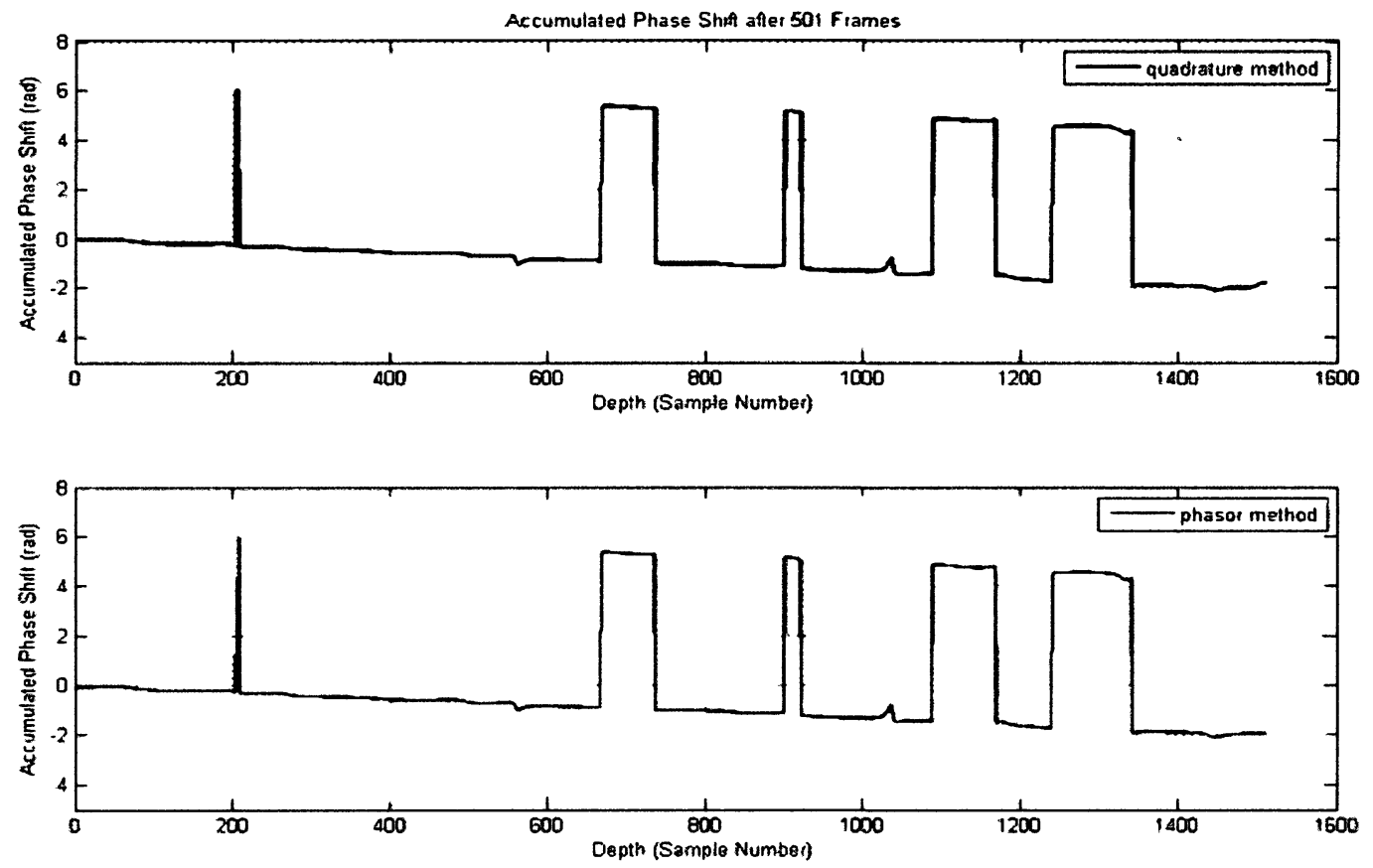

Figure 5.5 The accumulated phase shift after 501 frames, equivalent to $50 \mu \mathrm{m}$ displacement of the total sample volume in depth after compression. The top plot is obtained via the quadrature method while the bottom one is obtained by the phasor (reference) method. The phase shift increases with depth but jumps are appeared in this plot.

Figure 5.4 shows an increaing trend with no $2 \pi$ phase jumps. However, $2 \pi$ phase jumps are appeared in the corresponding accumulated phase shift plot where the number of frames is increased. Setting $\Delta d_{z}(N . m)$ to a greater value such as $50 \mu \mathrm{m}$, causes both the consecutive phase shift and the accumulated phase shift plots to show unexpected $2 \pi$ phase jumps at certain sample numbers.

Based on the observations, it is better to set $\Delta d_{z}(N . m)$ to $0.1 \mu m$ or a smaller value even though jumps will be observed in the corresponding accumulated phase shift result. However, by setting $\Delta d_{z}(N . m)$ to a very small value such as $0.1 \mu \mathrm{m}$, several frames will be needed in order to simulate the accumulated phase shift results. Therefore, in the simulation done in this work, 
$\Delta d_{z}(N, m)$ is assumed to be $50 \mu m$. In the following section, the reason behind $2 \pi$ phase jump creation will be explained.

\subsection{Cause(s) of $2 \pi$ Phase Jump Creation}

$2 \pi$ phase jump creation in the phase or phase shift plots stems from the limitation of phase measurement methods. The angle (phase) $\theta$ and its $2 \pi$-complements $(\theta \pm 2 k \pi)$ show the same points in the unit circle and thus, trigonometric functions of the angle $\theta$ and $\theta \pm 2 k \pi$ will give the same results. In phase measurement methods, the following equivalence is considered

$$
\theta \equiv \theta \pm 2 k \pi
$$

and only phase principal values, only those values that lie between $\pm \pi$ radians, will be extracted. The phase principal values are called "wrapped" phase values because the absolute phase (whatever it happens to be) is wrapped into the interval $(-\pi, \pi)$. The word "wrap" is referred to cycle discontinuities. The wrapped phase values at different points in depth (sample number) corresponding to time $t$ are obtained through a mathematical operation (wrapping process) on the actual phase of the signal as (Ghiglia \& Pritt, 1998).

$$
\theta_{\text {urapped }}(t)=\theta(t) \pm 2 \pi k(t)
$$

In this process, the actual phase $\theta(t)$ is wrapped into the interval $(-\pi, \pi)$ by $h:(t)$ which is an

integer function that forces $-\pi<\theta_{\text {wrapped }}(t)<\pi$. Accordingly, $\theta_{\text {urapped }}(t)$ will become a nonlinear function of $\theta(t)$. The process shown is Eq. (5.5) is the basis of all phase measurement methods. 
Wrapping process or simply the phase measurement method, causes a number of $2 \pi$ phase jumps to be observed in the measured phase information $\left(\theta_{\text {urapped }}(t)\right)$. These jumps could happen when the phase changes from a value around $-\pi+\varepsilon$ to a value close to $-\pi-\varepsilon$ and instead of $-\pi-\varepsilon$, the $2 \pi$ complement (which is equal to $\pi-\varepsilon$ ) is measured. This happens when in zplane noise causes movement between a complex number with small negative imaginary part and large negative real part to another complex number with small positive imaginary part and large negative real part (see Figure 5.6). Consequently, the phase which is measured by the $\arctan \left(\frac{I m a g}{\operatorname{Renl}}\right)$ is changed from a value greater than $-\pi$ to a value less than $+\pi$ and a $2 \pi$ phase jump will be created at these sample numbers.

In Matlab programming, the "angle" command is used for phase measurement with the same basis of operation mentioned above.

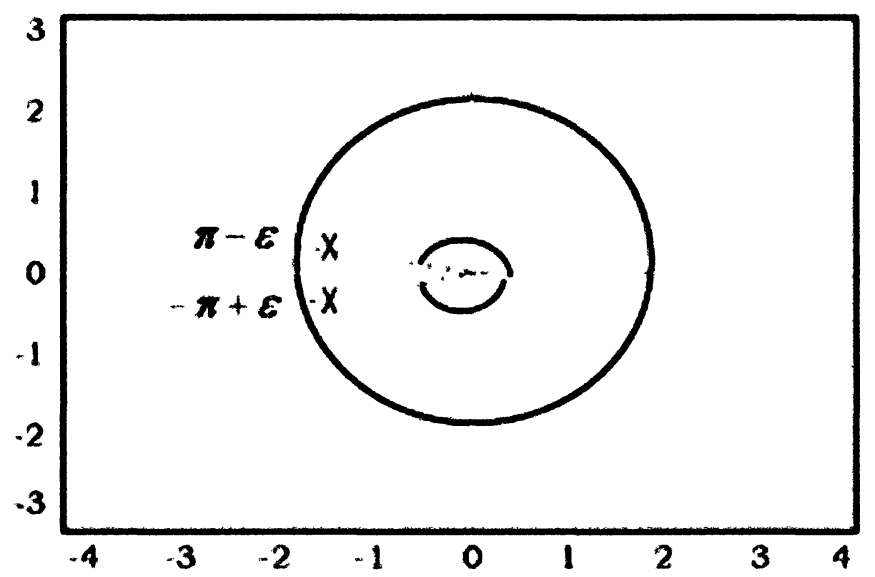

Figure 5.6 By moving from quadrant 3 to quadrant 2 (or vice versa) in the regions close to the unit circle, the magnitude of phase is changed from a value less than $-\pi$ to a value greater than $\pi$ (or vice versa) causing a $2 \pi$ phase jump to be created. 


\subsection{1 $\Delta d_{z}(N, m)$ Boundary to Avoid Phase Jumps}

Since this limitation is existed in phase measurement methods, in the quadrature technique application a restriction is made to avoid phase jumping. In this technique, $\Delta \varphi_{z}(N . m)$ is restricted to be less than $2 \pi$ which in turn, causes a boundary for instantaneous displacements of the entire volume $\left(\Delta d_{z}(N . m)\right)$.

Another representation of Eq. (5.1), that demonstrates the relationship between phase shift and instantaneous displacement, is

$$
\Delta d_{z}(n \cdot m)=\frac{\lambda}{4 \pi}\left(\Delta \varphi_{z}(n \cdot m)\right) . \quad n=1: N . m=1: M
$$

where $\lambda$ is the wavelength of the ultrasound transmitted signal. According to the Eq. (5.6), if we restrict $\Delta \varphi_{z}(N, m)$ to be less than $2 \pi, \Delta d_{z}(N . m)$ will also be limited to be less than the half of wavelength. In quadrature detection technique application, this restriction is made and the instantaneous displacement of the entire volume is assumed to be less than the half of wavelength.

The wavelength is calculated by (Prince \& Links, 2006)

$$
\lambda=\frac{c}{f_{c}}
$$

where $c$ and $f_{c}$ are the acoustic velocity and ultrasound center frequency, respectively. In the simulation, the acoustic velocity and ultrasound center frequency were assumed to be $1540 \mathrm{~m} / \mathrm{s}$ and $5 \mathrm{MHz}$, respectively. Therefore, the instantaneous displacement of the entire volume $\left(\Delta d_{z}(N, m)\right)$ is restricted to be less than $154 \mu m$, based on Eq. (5.7). 
This boundary was considered in section 5.1.1, when different values of $\Delta d_{z}(N, m)$ were examined. However, even by this consideration, with $\Delta d_{z}(N, m)$ is equal to $50 \mu m, 2 \pi$ phase jumps were observed in the resulted phase shift plots. It is due to the fact that each echo initially has a random phase which is shifted as a result of compression. At the position where the phase of a particular echo is close to $-\pi$ or $+\pi$, a small amount of displacement can cause the phase to $2 \pi$ jump, even though the compression is applied in a way that the maximum phase shift between RF signals be less than $2 \pi$.

\subsubsection{Phase Unwrapping}

Unwrapping process corrects phase angles to produce smoother phase plots. In this process, it is aimed to obtain an estimate of the actual phase $(\hat{\theta}(t))$ from the unwrapped version of $\theta_{\text {urapped }}(t)$. In other words, the measured wrapped values $\theta_{\text {urapped }}(t)$ must be unwrapped somehow to estimate $\theta(t)$ (actual phase). In unwrapping process, the $2 \pi$ jumps that are created in the measured phase results are fixed by adding the appropriate multiple of $\pm 2 \pi$ at the position of jumps (Ghiglia \& Pritt, 1998). This function is availabe in Matlab as the "unwrap" command. In Figure 5.9, the phase shift between the first two (pre- and post-compression) RF signals along with the unwrapped version of it is represented. 
Consecutive Phase Shit between Frames 1 and 2
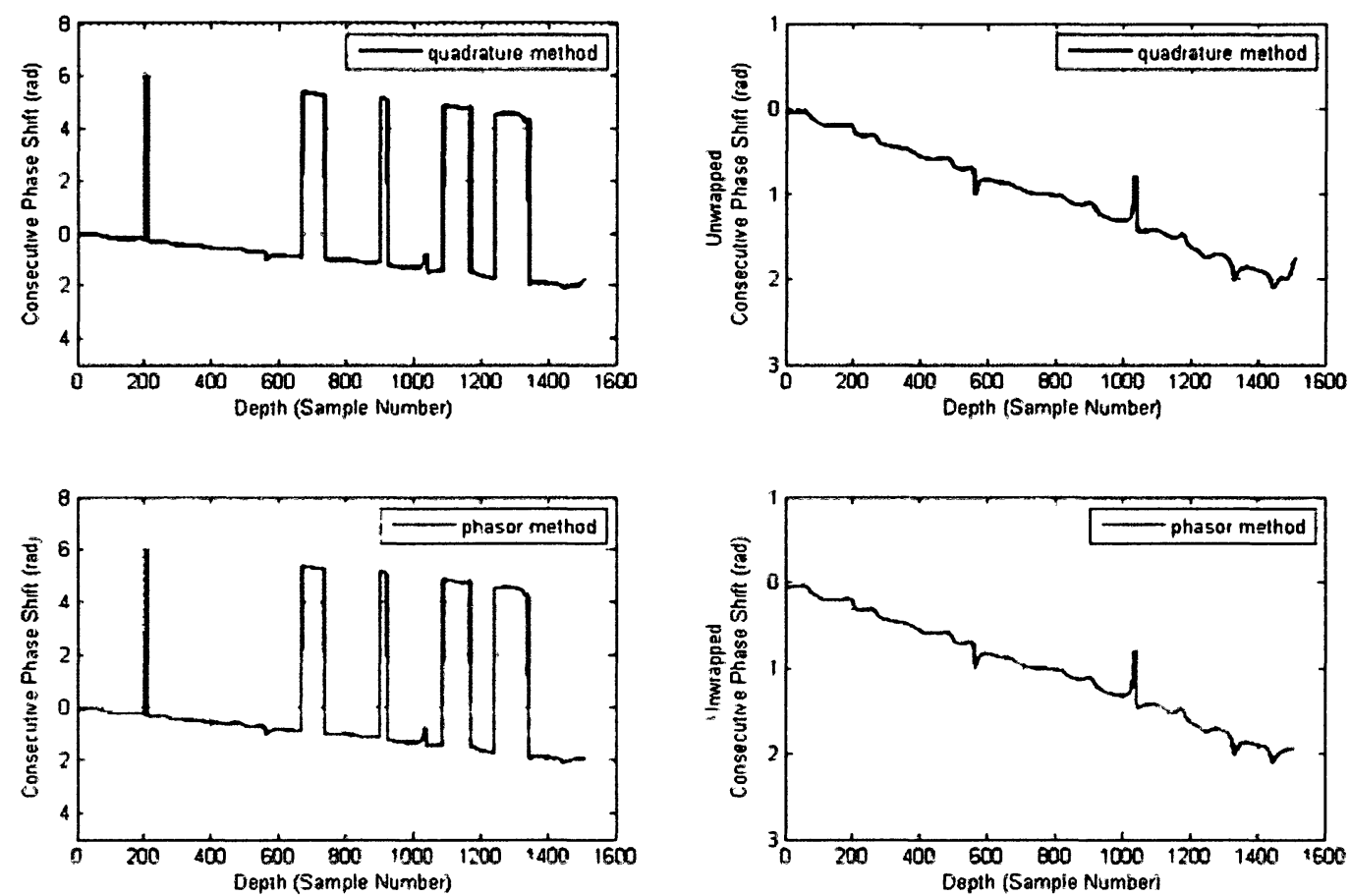

Figure 5.7 Phase shift between the first and second (pre- and post-compression) RF signals (left); quadrature method (top left); phasor (reference) method (bottom left); corresponding unwrapped phase shift between these RF signals (right)

As it can be seen, the phase jumps of $2 \pi$ are fixed in the unwrapped phase shift results. However, unwrapping is not fully reliable and does not always give a good result such as the one represented in Figure 5.7.

\subsubsection{Failure of the Unwrapping Process}

Phase unwrapping implementation is in fact, problematic for several reasons. To work with actual signals in computer, they are required to be sampled and converted to digital signals. Thus, continuous functions become a sequence of samples. According to the sampling theory, the sampled data constitute an imperfect representation of the continuous signal since the sampled data measure the continuous signal at discrete points in time, and they may span a 
limited extent of the total signal duration. Therefore, an inadequate sampling rate or duration can prevent reconstruction of the continuous signal to a satisfactory level of reliability. Most phase unwrapping problems happen when one moves from the continuous to the discrete domain. In continuous domain, there is only on way to unwrap phase, and the unwrapped result is unique. In discrete domain, however, certain assumption must be made (e.g., the assumption of nonaliasing). In some way, phase unwrapping is equivalent to recovering the underlying continuous wrapped phase from the discrete wrapped phase that has been sampled (Ghiglia \& Pritt, 1998).

It can be observed that the unwrapping process fails to fix the less than $2 \pi$ phase jumps. This happens when the slope of the phase shift plot becomes relatively steeper between certain consecutive samples and the sampling rate is not enough to cover more samples. An example of a less than $2 \pi$ phase jump is shown in Figure 5.8. In this figure, the phase shift between the $5^{\text {th }}$ RF signal pairs along with its unwrapped version is plotted. As it is shown in this figure, unwrap fails to fix the created jump. 

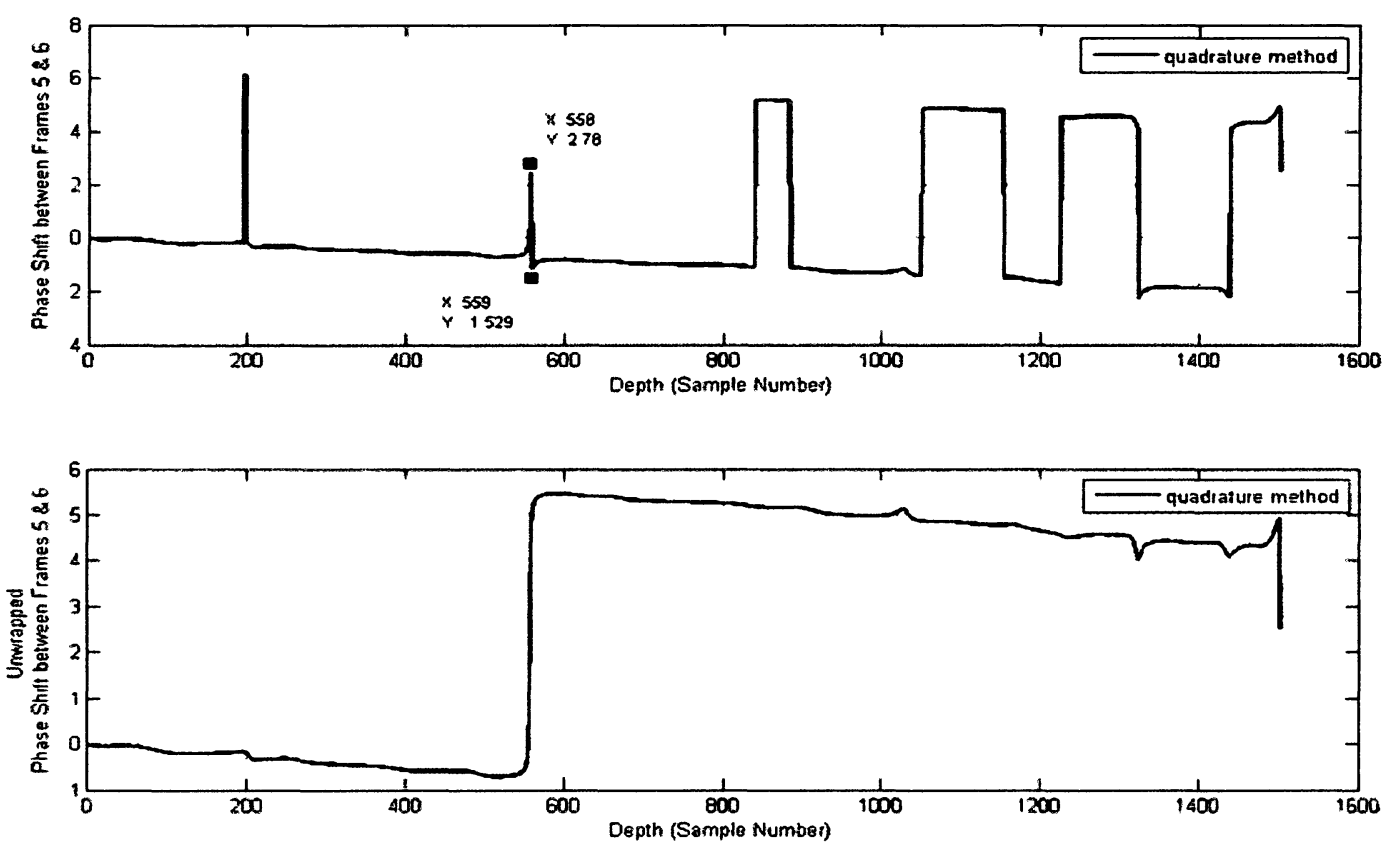

Figure 5.8 The phase shift between the $5^{\text {th }}$ RF signal pairs resulted by the quadrature method (top) and the unwrapped version of it (bottom). At the sample number of 558, a $\pi$ phase jump is created. This jump is not fixed in the unwrapped phase shift plot.

In the z-plane this happens when noise causes movement between a complex number with small positive imaginary part and small negative real part to another complex number with small negative imaginary part and small positive real part. Consequently, the phase which is measured by the $\arctan \left(\frac{I \text { mag }}{\text { Real }}\right)$ is changed between values in quadrant 2 and 4 and thus, a phase jump of $\pi$ (less than $2 \pi$ ) will be created at these sample numbers (see Figure 5.9). The real and imaginary parts of the $5^{\text {th }}$ and $6^{\text {th }}$ RF signals are shown in Figures 5.10, and 5.11. At the position where a $\pi$ phase jump is created, the value of the $5^{\text {th }} \mathrm{RF}$ signal is $(-0.01+\jmath 0.04)$ while it is equal to $(0.12-j 0.19)$ in the $6^{\text {th }}$ RF signal. This causes a phase shift of about $\pi$ to be observed in the phase shift plot. 


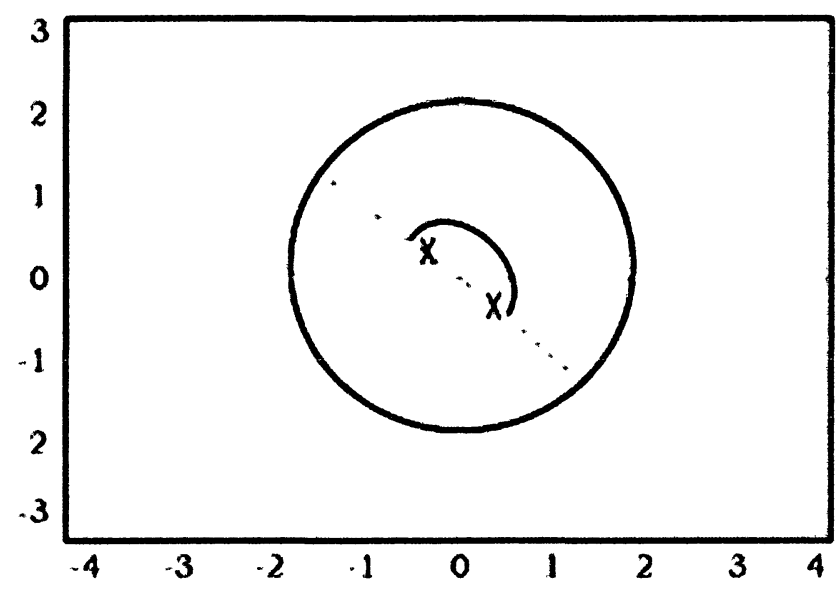

Figure 5.9 By moving from quadrant 2 to 4 (or vice versa) in the regions close to the center of the unit circle, a $\pi$ phase jump will be created.

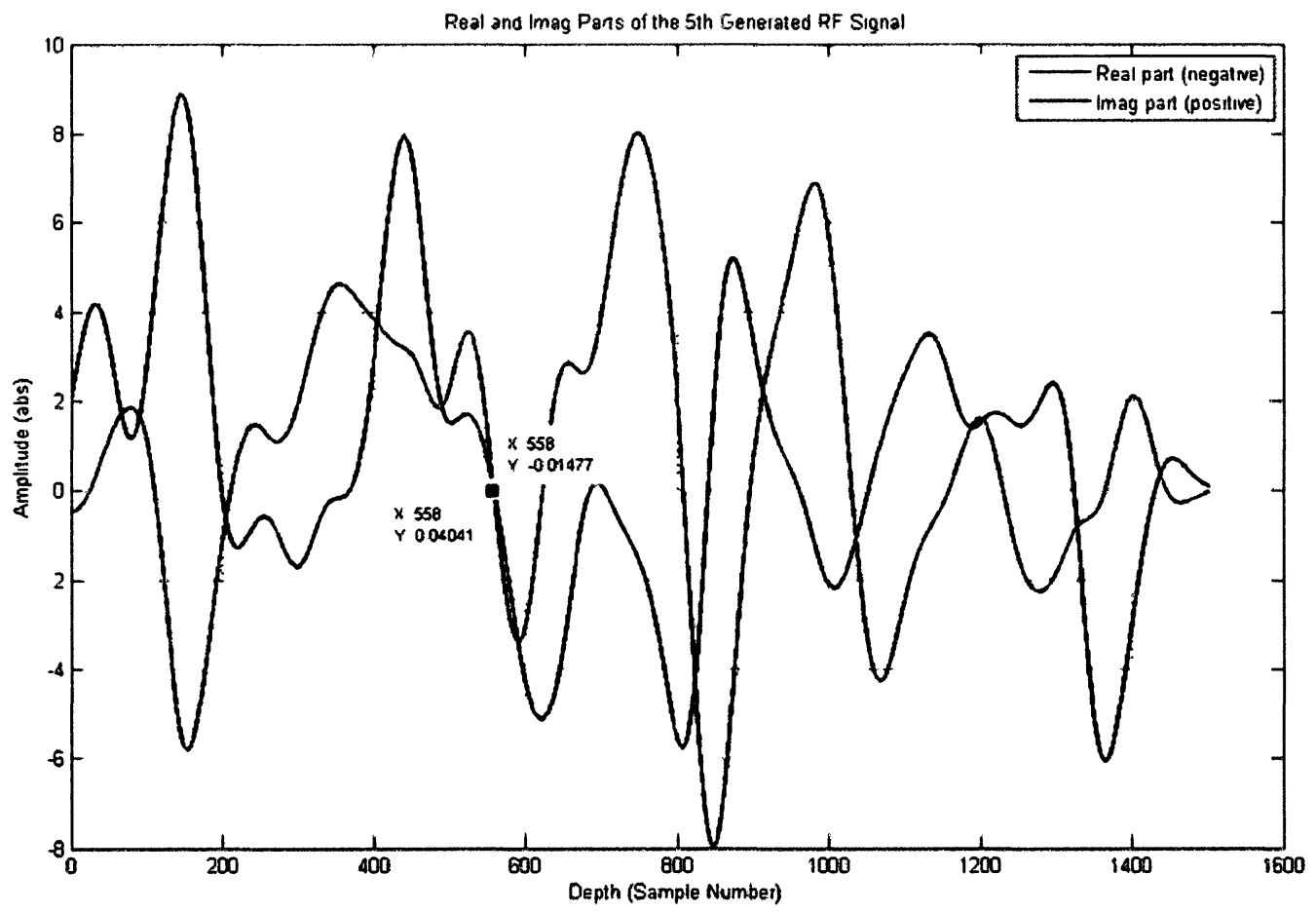

Figure 5.10 Real (red) and imaginary (blue) parts of the $5^{\text {th }}$ simulated RF signal. At the sample number of 558, the value of the RF signal is $-0.01+j 0.04$, which is a complex number close to the center of the unit circle, in the quadrant 2 of the z-plane (small real and imaginary parts). 


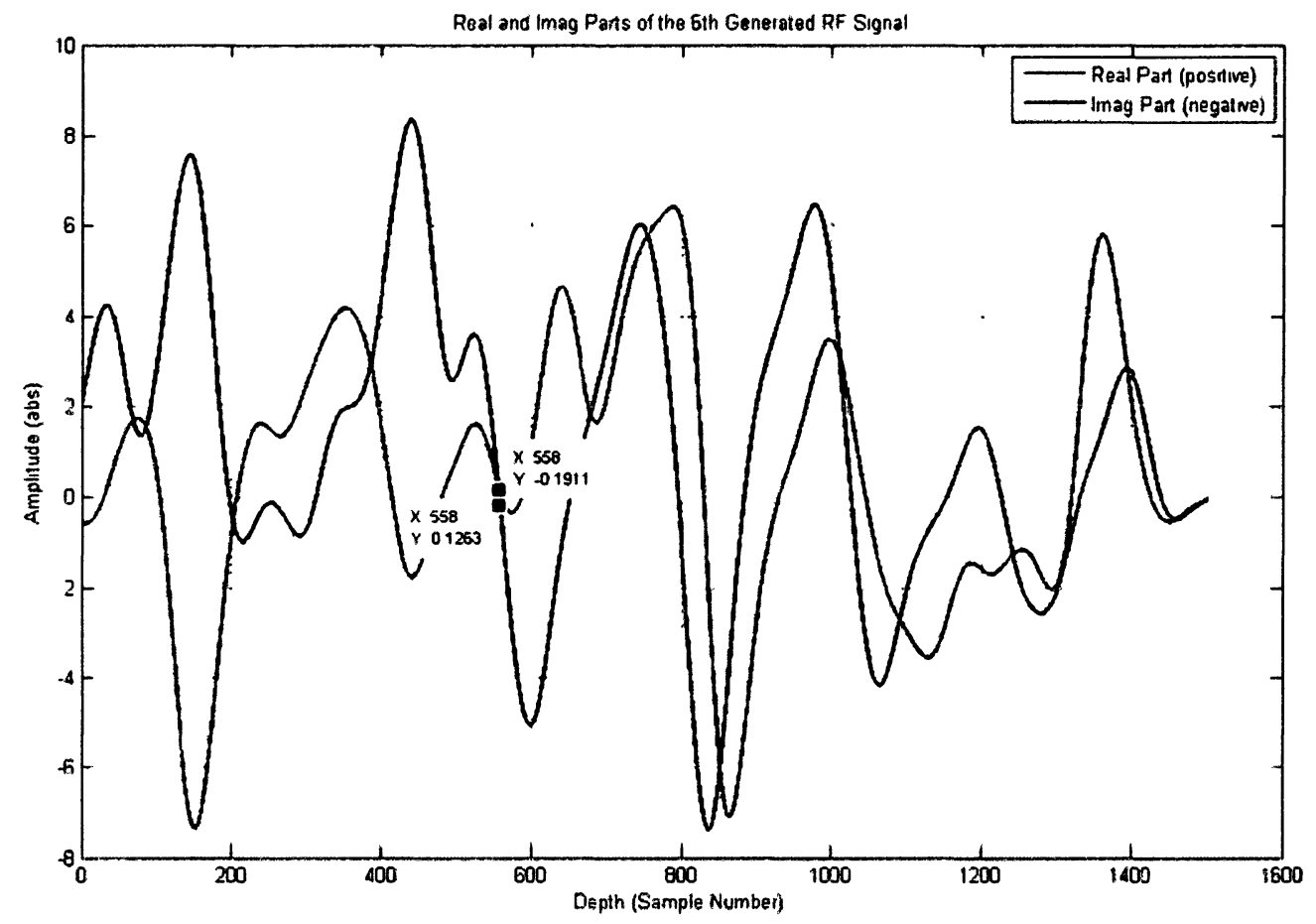

Figure 5.11 Real (red) and imaginary (blue) parts of the $6^{\text {th }}$ simulated RF signal. At the sample number of 558, the value of the RF signal is $0.12-j 0.19$, which is a complex number close to the center of the unit circle, in the quadrant 4 of the z-plane (small real and imaginary parts).

\subsection{Extension of the Phase Shift Simulation}

The simulation with $\Delta d_{z}(N . m)$ equal to $50 \mu m$ was repeated to create other pairs of RF signals. The resulted phase shift plots were similar except that in each plot, the spikes ( $2 \pi$ phase jumps) were slightly shifted to the left compare to that of the previously captured RF signal pairs. This happened because the signals were compressed and became shorter during the force application causing the positions of scatterers to be changed.

By continuing the simulation and creating several consecutive phase shift plots, it was then possible to plot the accumulative phase shift results. As an example, the accumulated phase shift after 5 frames (corresponding to the $200 \mu m$ displacement of the volume) along with the first 4 
consecutive phase shift plots are shown in Figure 5.12. Since the results of both the quadrature and reference methods were similar, in this figure only the quadrature method's outcomes are shown.

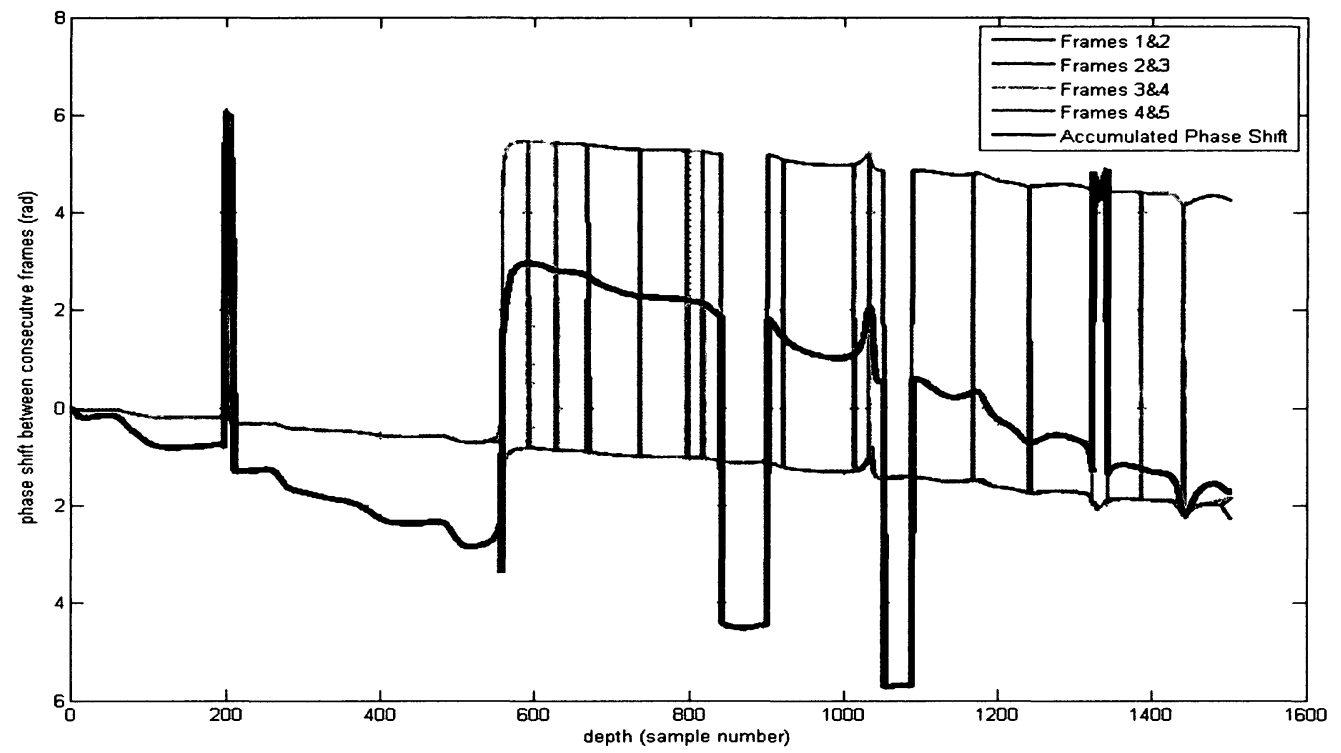

Figure 5.12 The accumulated phase shift after 5 frames, equivalent to the total displacement of $200 \mu m$ (black). The resulted consecutive phase shift plots corresponding to the first, second, third, and forth RF signal pairs are shown in blue, red, green, and purple, respectively. Certain spikes that are created in each of these plots are shifted to some extend as the number of frames goes up.

In Figure 5.12, the magnitude of the accumulated phase shift increases with depth. However, it has wider jumps compare to the consecutive phase shift plots. Indeed, all spikes in consecutive phase shift plots will be added up resulting in wider jumps in the corresponding accumulated phase shift plots. 


\subsubsection{Accumulated Phase Shift Equivalent to $1 \mathrm{~mm}$ Displacement of the Volume}

As mentioned earlier, the total accumulated displacement of the sample volume after compression is assumed to be $1 \mathrm{~mm}$. Corresponding accumulated phase shift is resulted after generation of 21 frames. In Figure 5.13, this accumulated phase shift is represented.

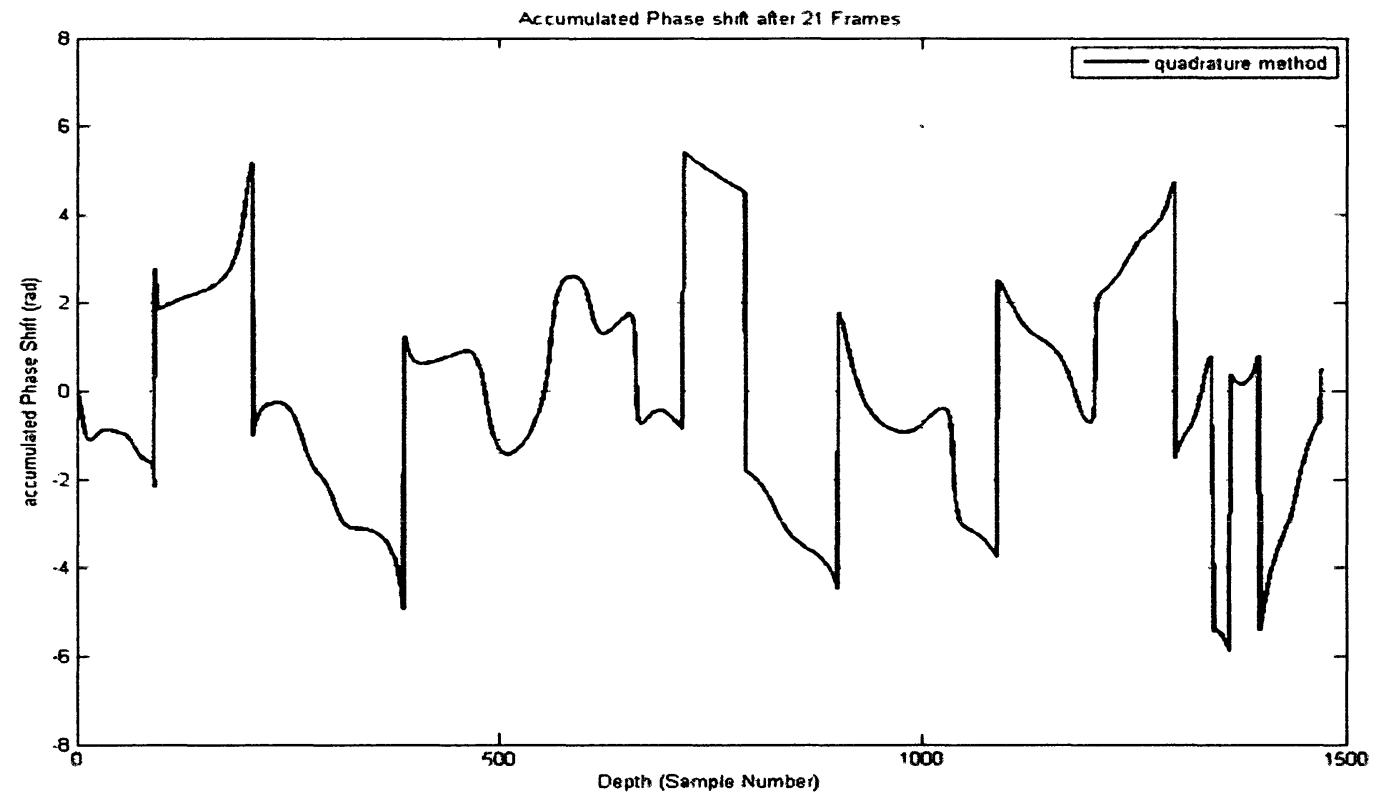

Figure 5.13 The accumulated phase shift after 21 frames, equivalent to $1 \mathrm{~mm}$ displacement of the sample volume after compression. This accumulated phase shift plot is resulted by the quadrature method. As it is shown in this figure, the linearly increasing trend is not observable any longer due to appearance of more jumps.

Figure 5.13 no longer shows the increasing trend of the phase shift plot. The number of jumps created in it is also increases, significantly. As the number of frames increase, the magnitude of the accumulated phase shift increases, spikes ( $2 \pi$ phase jumps) are added up, and the jumps become wider. By further increasing the number of frames, spikes will be continually created over the wider jumps and so on. Repeatedly generating the spikes will end with an unexpected result shown in Figure 5.13. Unwrapping process can not entirely improve the accumulated 
phase shift plot shown in Figure 5.13 due to the same reasons explained earlier. The unwrapped version of this plot is represented in Figure 5.14.

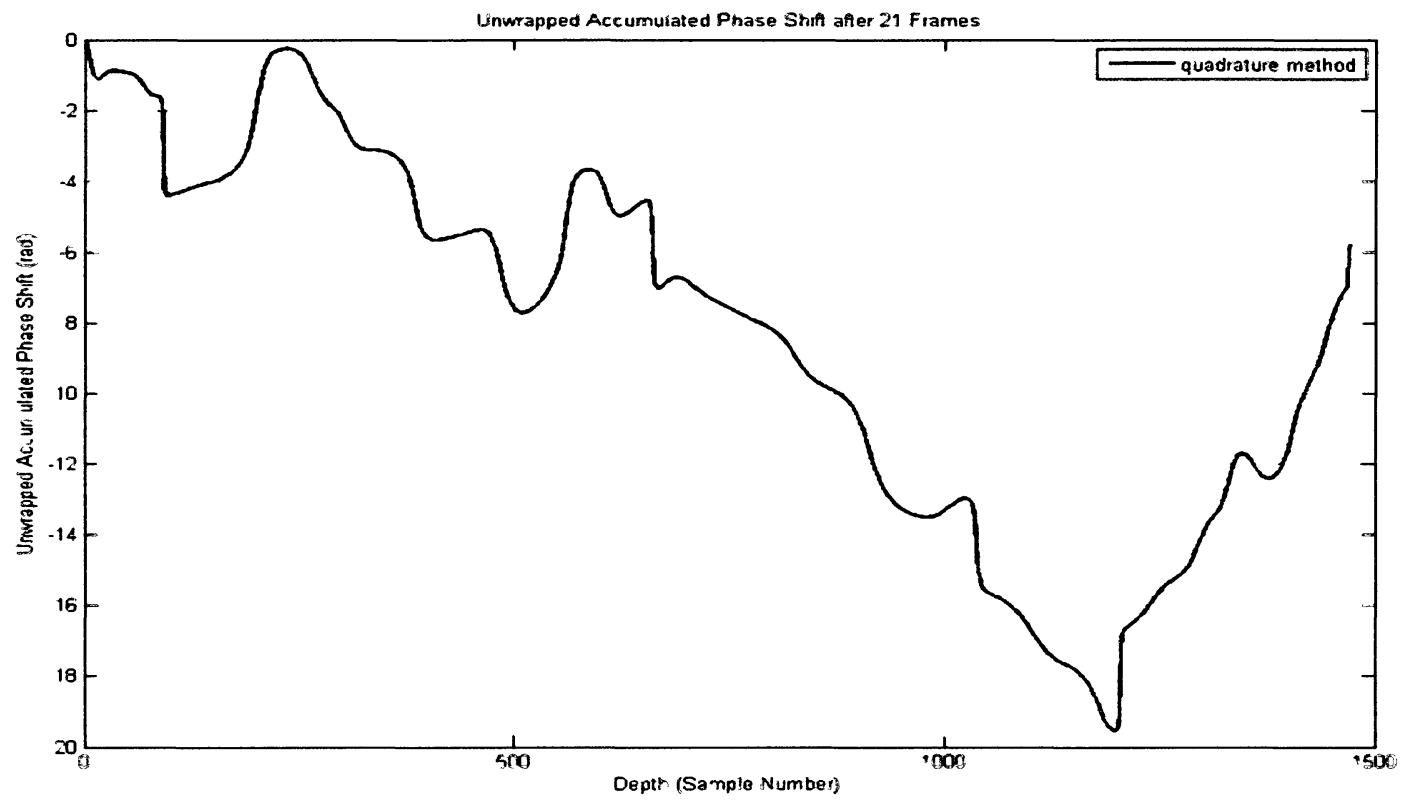

Figure 5.14 The unwrapped version of the accumulated phase shift equivalent to $1 \mathrm{~mm}$ displacement of the total sample volume after compression. Again this plot is resulted by quadrature method application.

\subsection{Quadrature Technique Performance in Different Conditions}

So far, it has been observed that the quadrature phase detection technique functions in accordance with the phasor (reference) method. The next step is to see how reliable the quadrature technique is and in what circumstances it will function less accurate. To see this, certain parameters such as the signal to noise ratio (SNR) of the RF signals, number of scatterers $(L)$ in the sample volume, and the bandwidth $(\beta)$ of echoes were varied. In the following, the effect of these variations on the quadrature resulted phase shift plots are outlined. 


\subsubsection{SNR Parameter Effect}

To examine the effect of the SNR parameter on the quadrature phase estimations, noise needed to be incorporated in the simulation. This was done by adding white Gaussian noise to the received RF signals. This choice was made based on the nature of noises that are involved with the ultrasound signals.

Different types of noise can be considered when dealing with ultrasound signals. Thermal and quantization noise are the two examples. The former is the electronic noise generated by the thermal agitation of the charge carriers (electrons) inside an electrical conductor, which can come from the ultrasound machine. Thermal noise is approximately white, and thus it can be simulated as the white Gaussian noise. Quantization noise is another type that can be introduced by quantization in the signal processing. The difference between the actual analog value and quantized digital value due is called quantization error. This error is due either to rounding or truncation. The error is sometimes considered as an additional random signal called quantization noise. This type of noise is also can be simulated by white Gaussian noise due to its random nature.

In so far represented figures, white Gaussian noise was added to the simulated received RF signals such that the signal to noise ratio (SNR) becomes $40 \mathrm{~dB}$. The resulted consecutive phase shift between the first two RF signals when the SNR is $40 \mathrm{~dB}$ is represented in Figure 5.3. In the next two figures, this phase shift is shown when the SNR level is reduced to $10 \mathrm{~dB}$ and $3 \mathrm{~dB}$, respectively. 

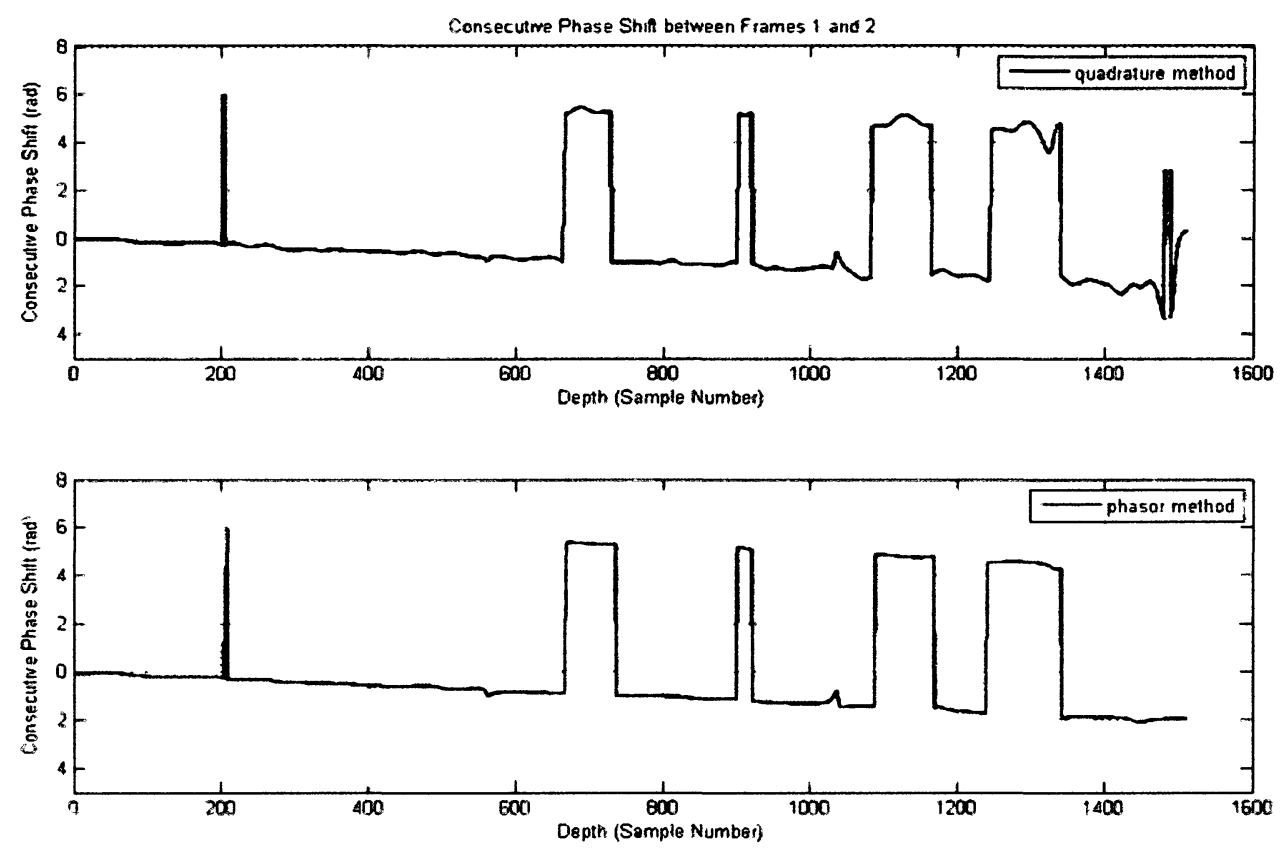

Figure 5.15 Phase shift between the first two (pre- and post-compression) RF signals with the SNR of $10 \mathrm{~dB}$. The top and bottom plots are the phase shift plots obtained by the quadrature and phasor methods, respectively.
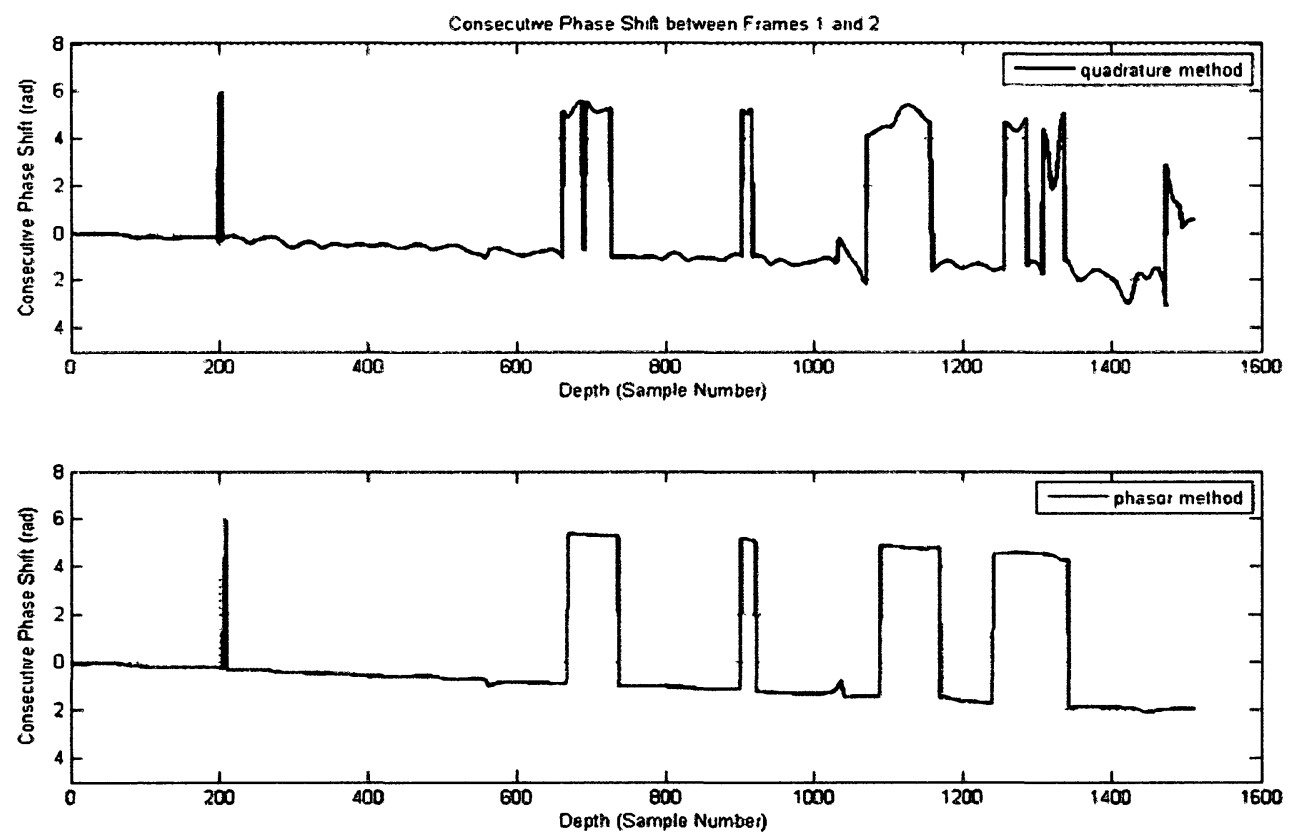

Figure 5.16 Phase shift between the first two (pre- and post-compression) RF signals with the SNR of $3 \mathrm{~dB}$. The top and bottom plots are the phase shift results obtained by the quadrature and phasor methods, respectively. 
In Figures 5.15 and 5.16, the phase shift plots obtained by the quadrature and phasor methods are no longer matched. This deviation is due to the fact that, the quadrature technique represents a noisy phase shift and its accuracy is reduced to some extent. Therefore, the SNR is a parameter that can affect the quadrature technique performance and its phase results.

In the simulation, white Gaussian noise was added to the received RF signals such that the signal to noise ratio (SNR) varied from $0 \mathrm{~dB}$ to $40 \mathrm{~dB}$. Then, the phase shift error (misfit between the phase shift plots resulted from the qudrature technique and phasor method) was calculated (Figure 5.17). Throughout this thesis, this error is called as the quadrature phase shift error and is shown by $\left(\Delta \varepsilon_{1}\right)$. Symbol $\varepsilon_{1}$ is used to show the quadrature phase error.

The range of 0 to $40 \mathrm{~dB}$ for the SNR level was chosen based on considering the amplitude spectrum of a particular real (measured) received RF signal. The amplitude of the measured RF signal around its center frequency $\left(f_{c}\right)$ was found to be 250 while the amplitude of speckle echoes, which are sometimes considered as noise, was found to be around 10. Normally, the amplitude of noise is less than that of speckle echoes. Therefore, it was assumed that the amplitude of noise is a value between 1 and 10. The SNR of measured RF signal around its center frequency and at other frequencies (corresponding to the speckle echoes) was then calculated by

$$
\mathrm{SNR}=20 \log \left(\frac{A_{\text {signal }}}{A_{\text {noise }}}\right)
$$

where $A_{\text {signal }}$ and $A_{\text {noise }}$ are the signal and noise amplitudes and SNR is in measured in $\mathrm{dB}$. Based on the measured RF signal's amplitude at different frequencies, the range of SNR found to be between 0 and $34 \mathrm{~dB}$ which was rounded to 0 to $40 \mathrm{~dB}$ in our simulation. 


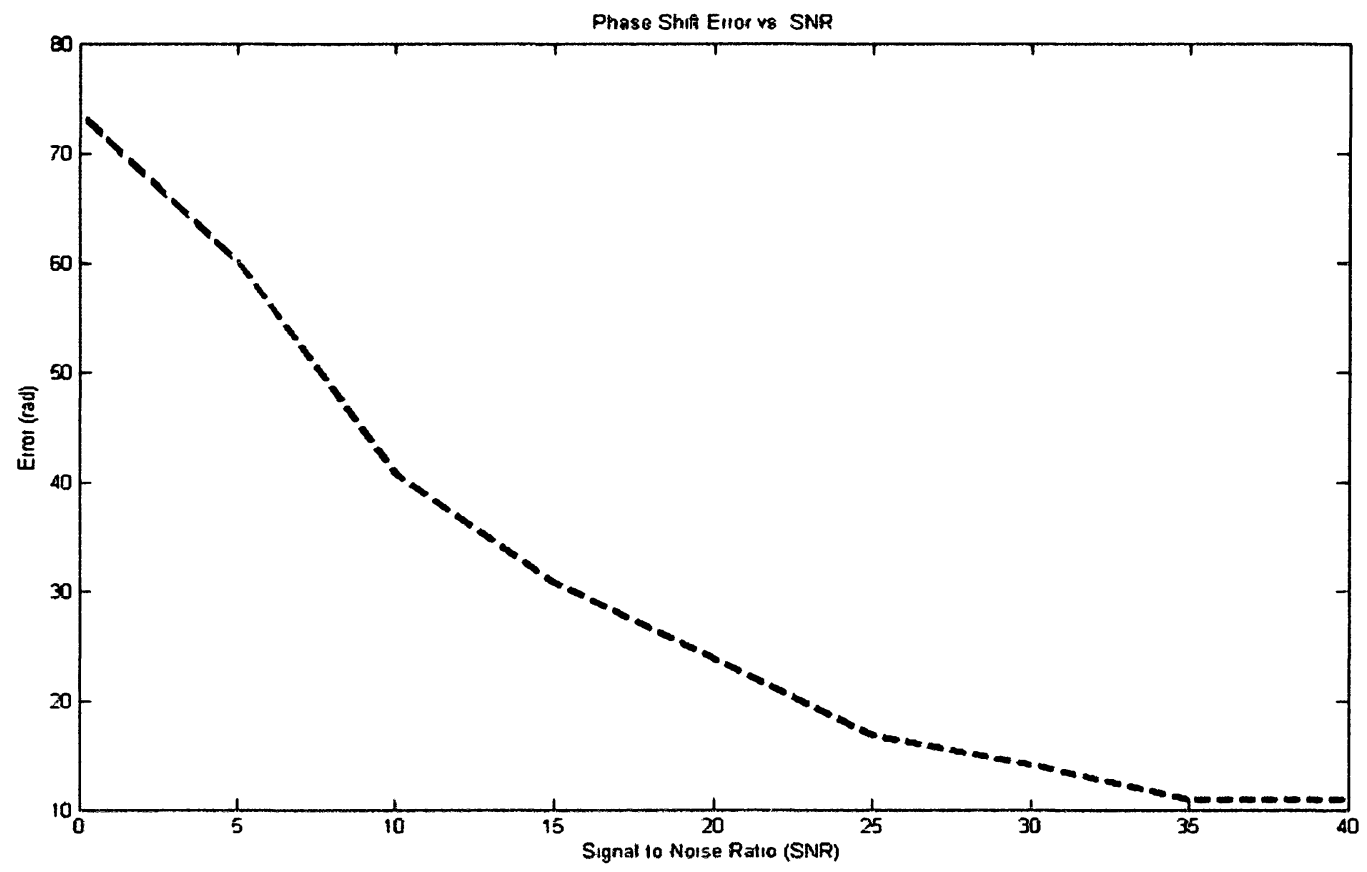

Figure 5.17 Quadrature phase shift error $\left(\Delta \varepsilon_{1}\right)$ of the first RF signal pairs vs. the signal to noise ratio (SNR).

It can be seen from the results that the quadrature detection technique used could be accurate if reasonable level of SNR is obtained. However, if this level is not achieved then the resulted phase shift outcomes will become less accurate.

\subsubsection{Number of Scatterers $(L)$ Parameter Effect}

Second parameter which seemed to affect the quadrature method was the number of scatterers. In so far represented figures, the number of scatterers has been assumed to be small $(L=111)$. Figure 5.18 is resulted when $L$ is increased to 1055 which is a large number according to (Narayanan, Shankar, \& Reid, 1994). 

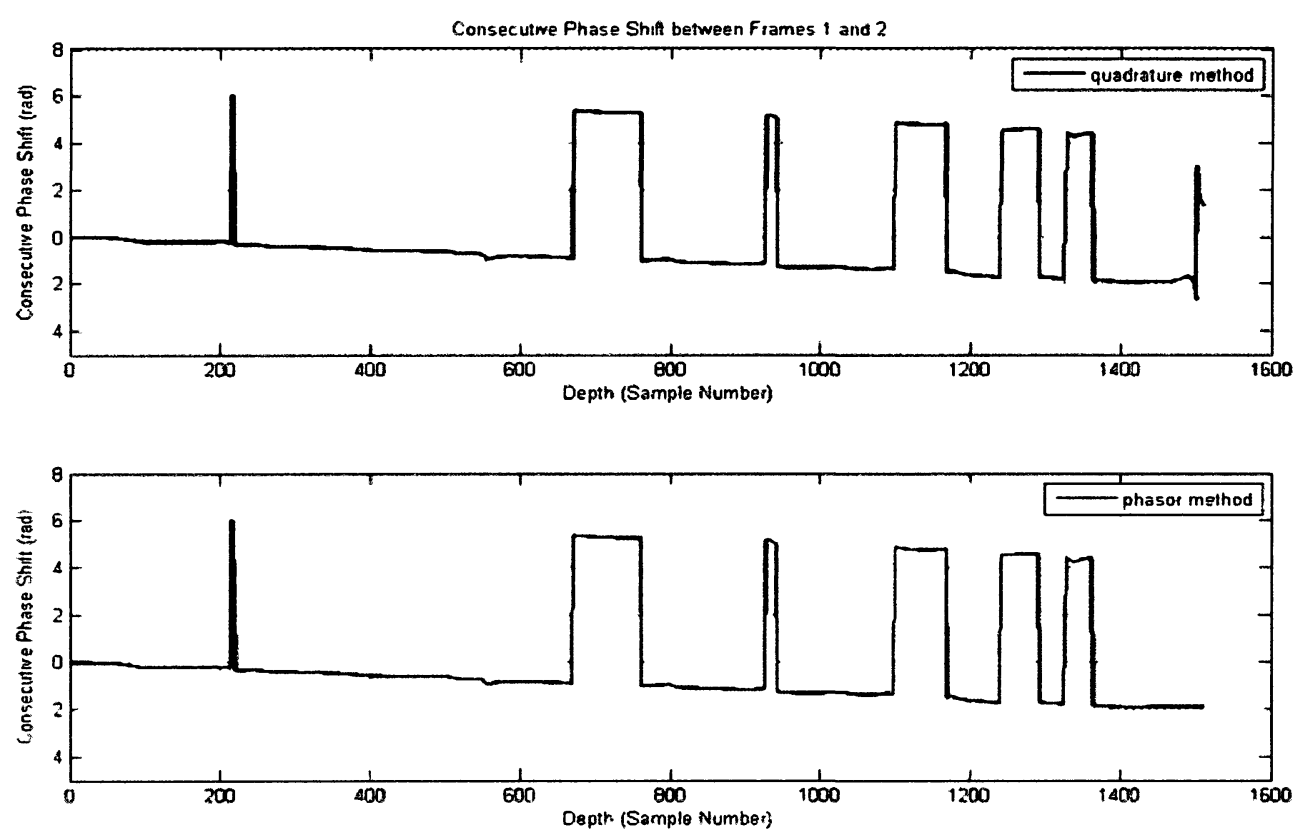

Figure 5.18 Phase shift between the first two consecutive (pre- and post-compression) RF signals when the number of scatteres in the sample volume is large (1055). The top and bottom plots are the phase shift results obtained by the quadrature and phasor methods, respectively.

In Figure 5.18, the phase shift plots obtained by both methods are almost similar. This shows that the number of scatterers is not a dominant parameter affecting the quadrature method performance or its outcomes.

In the simulation, the number of scatterers $(L)$ in the sample volume varied from 117 to 1055 . Then, the phase shift error $\left(\Delta \varepsilon_{1}\right)$ was calculated (Figure 5.19). Although by changing the number of scatterers the phase shift error varied, this variation was not huge and remained roughly between 0 to $15 \mathrm{rad}$. Therefore, the number of scatterers is considered as a parameter that affects the quadrature technique, insignificantly. 


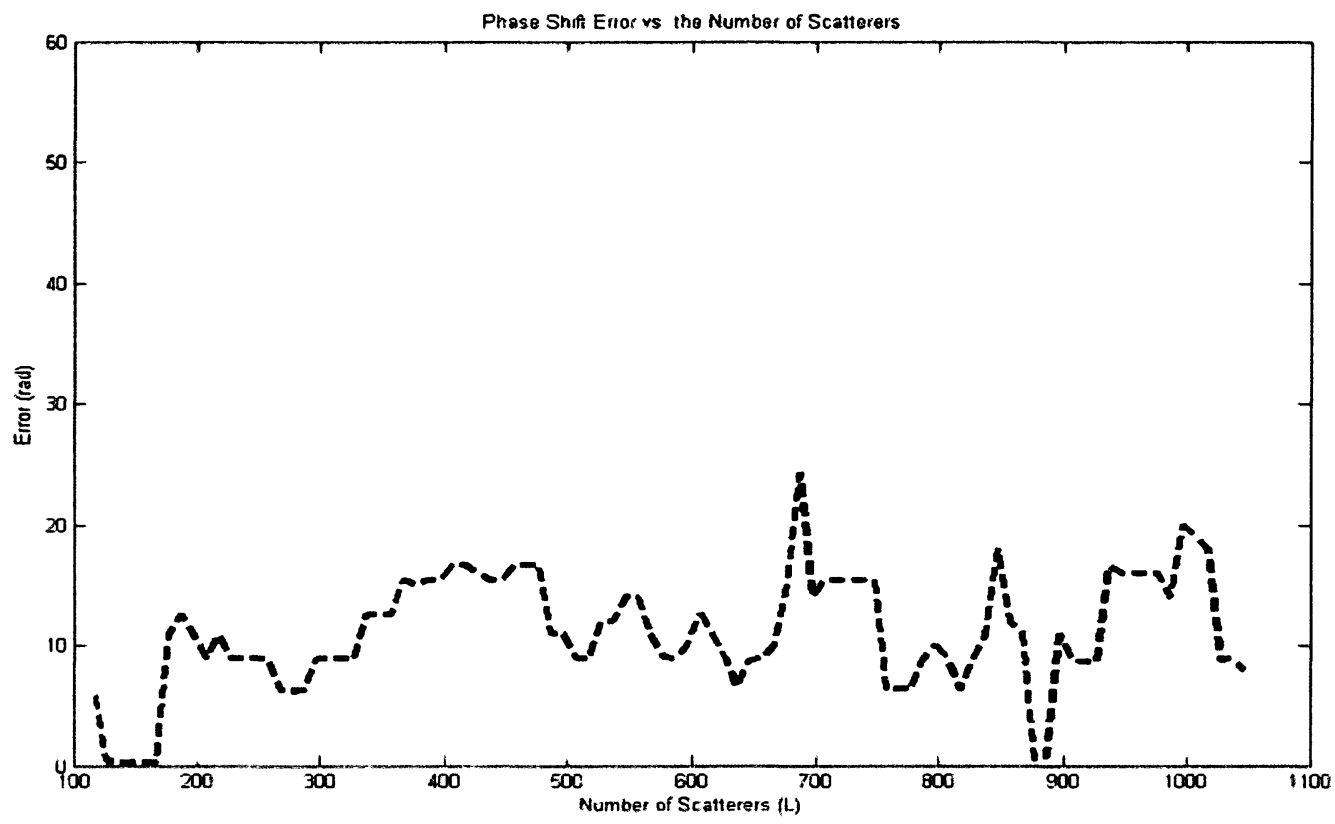

Figure 5.19 Quadrature phase shift error $\left(\Delta \varepsilon_{1}\right)$ of the first RF signal pairs vs. the number of scatterers in the sample volume.

\subsubsection{Bandwidth Effect}

Another parameter seemed to affect the results of the quadrature method was the bandwidth of echoes. In so far represented figures, the bandwidth of echoes has been assumed to be $0.8 \mathrm{MHz}$ (\%16). With this bandwidth, the quadrature and phasor methods resulted in the same phase and phase shift plots. In the following figures, the value of bandwidth is changed and the quadrature phase shift results are outlined. 

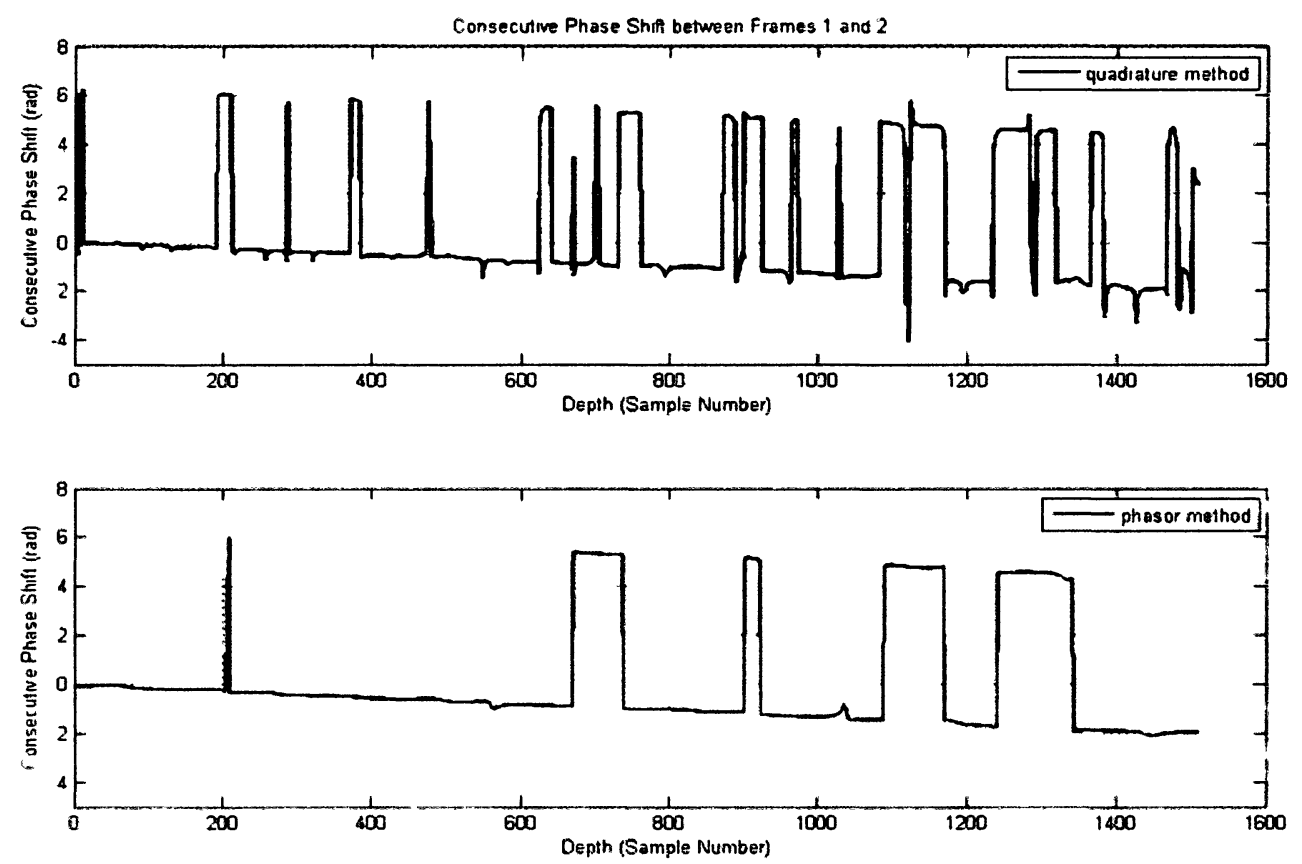

Figure 5.20 Phase shift between the first two consecutive (pre- and post-compression) RF signals when the bandwidth of echoes is $2.25 \mathrm{MHz}$. The top and bottom plots are the phase shift results obtained by the quadrature and phasor methods, respectively.

By increasing the bandwidth of signal, the phase shift plots obtained by the quadrature and phasor methods will no longer be similar. In the next figure, the bandwidth is reduced to 4.2 $\mathrm{MHz}(\% 75)$. 

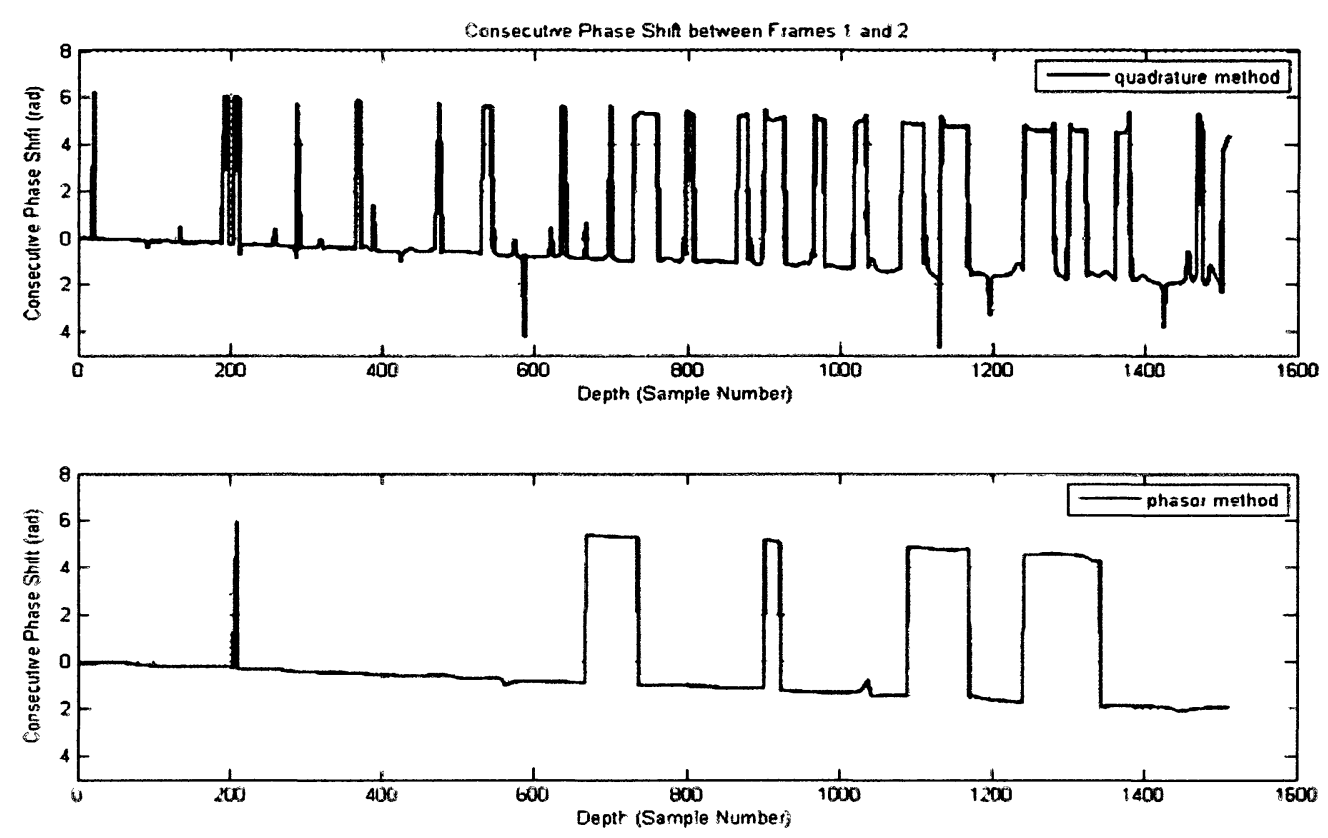

Figure 5.21 Phase shift between the first two consecutive (pre- and post-compression) RF signals when the bandwidth of echoes is $4.2 \mathrm{MHz}$. The top and bottom plots are the phase shift results obtained by the quadrature and phasor methods, respectively. The resulted phase shift plots are deviates more when the bandwidth is changed to 4.2 MHz.

By increasing the bandwidth of echoes, more jumps will be appeared in the quadrature outcomes and the phase shift plots deviate more from those obtained from the reference method. Therefore, bandwidth of the received RF signal (or its constitutive echoes) is considered as a parameter that affects the quadrature method and its outcome, significantly.

In contrast with the signals in communication systems, increasing the bandwidth of the ultrasound received signals will not give more information about them. In communication systems, signals are wideband and thus, by increasing the bandwidth more information will be obtained. However, ultrasound received RF signals are more narrowband and more concentrated around their center frequencies $\left(f_{c}\right)$, which is varied with depth and attenuation. The amplitude of the signal at other frequencies will give information about noise and speckle echoes which are also considered as noise. In our simulation, the center frequency of the received RF signals was 
considered as a constant value $(5 \mathrm{MHz})$. Therefore, by increasing the bandwidth of the simulated RF signal, more noise will be considered and the phase outcomes will become poorer.

Bandwidth of the simulated received RF signal was varied from $0.8 \mathrm{MHz}$ to $5 \mathrm{MHz}$ and the quadrature phase shift error $\left(\Delta \varepsilon_{1}\right)$ was calculated (Figure 5.22). The quadrature technique could be accurate if reasonable value for the RF signals' bandwidth is selected. However, the range of this selection is limit. With bandwidth equal to $0.8 \mathrm{MHz}$, the phase shift error $\left(\Delta \varepsilon_{1}\right)$ is not large (10.89 rad); however, it increases considerably with increases in the bandwidth value.

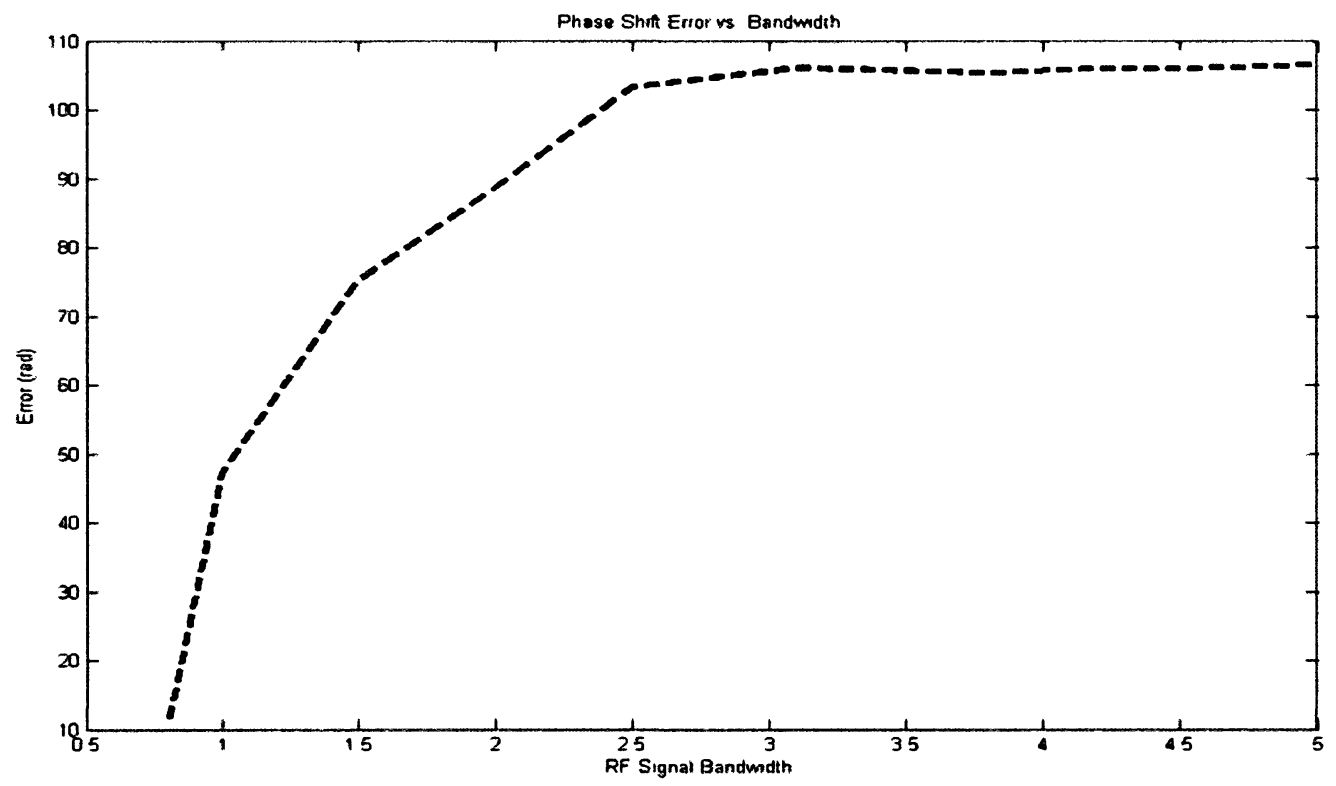

Figure 5.22 Quadrature phase shift error $\left(\Delta \varepsilon_{1}\right)$ of the first RF signal pairs vs. the bandwidth of the received RF signal.

In the next chapter, certain algorithm will be explained which is able to reconstruct the phase (phase shift) results obtained via the quadrature method. Among different parameters affecting the quadrature technique, SNR is chosen for the further examinations. Studying of other parameters is left as a future work. 


\section{Chapter 6}

\section{Inverse Problem Techniques}

This chapter provides a general description of inverse problems with the emphasis on nonlinear category and associated solving techniques. It describes a novel approach for ultrasound phase reconstruction by means of an algorithm known as Gauss-Newton (GN). In addition, it represents some phase plots reconstructed by GN algorithm. This chapter closes with the effect of regularization parameter on the phase reconstruction algorithm.

As explained in chapter 4 , the quadrature phase detection technique is normally used to extract the phase information of ultrasound received RF signals. It was observed that in certain situations, such as when the SNR is low, the results of this technique will become less accurate. It was also observed that phase jumps of less than $2 \pi$ appeared in the quadarture phase shift outcomes cannot be fixed by unwrapping process. Therefore, it is desired to find an algorithm or technique to reconstruct the less accurate phase outcomes resulted by the quadrature method. Inverse problem solving techniques seems to be able to solve these problems. In the following section, general inverse problem will be explained.

A small modification has been made in the notations used before. Here, symbol $\mathbf{d}$ is the

vector of observed data and $d_{2}$ is the $i^{\text {th }}$ element of it. It doesn't represent the local displacement discussed in chapter 2 . 


\subsection{Inverse Problems}

Scientifically, it is desired to relate physical parameters characterizing a model $\mathbf{m}$ to collected observations or data $\mathbf{d}$. The fundamental physics are usually understood, so a function, $\mathrm{G}$ relating $\mathbf{m}$ and $\mathbf{d}$ is specified as

$$
\mathbf{G}(\mathbf{m})=\mathbf{d}
$$

Data $\mathbf{d}$ may be a function of time or space, or may be a set of discrete observations. There is however, an issue that the actual observations always contain some amount of noise. Thus, data is imagined as noiseless observations from a "perfect" experiment, $\mathbf{d}_{\text {true }}$, plus a noise constituent $\eta$

$$
\begin{aligned}
\mathbf{d} & =\mathbf{G}\left(\mathbf{m}_{\text {true }}\right)+\eta \\
& =\mathbf{d}_{\text {true }}+\eta
\end{aligned}
$$

where $\mathrm{d}_{\text {true }}$ exactly satisfies the Eq. (6.1) for $\mathbf{m}$ equal to the true model represented by $\mathbf{m}_{\text {true }}$. It is often the case that a solution for $\mathbf{m}$ that is affected by a little noise amplitude $\eta$, can have little or no similarity to $\mathbf{m}_{\text {true }}$ (Aster, Thurber, \& Borchers, 2005).

\subsubsection{Terminology}

In modeling terminology, there is a disagreement between mathematicians and other scientists. Applied mathematicians generally refer to $\mathbf{G}(\mathbf{m})=\mathbf{d}$ as the "mathematical model" and to $\mathbf{m}$ as the "parameters" while scientists usually refer to $\mathbf{G}$ as the "forward operator" and to $\mathbf{m}$ as the 
"model." In this thesis, $\mathbf{m}$ is referred as the "model" while the equations of $\mathbf{G}(\mathbf{m})=\mathbf{d}$ are considered as the "mathematical model" (Aster et al., 2005).

\subsubsection{Inverse Problem Definition}

By defining the relation in (6.1), the inverse problem is to find $\mathbf{m}$ given $\mathbf{d}$ while the forward problem is to find $\mathbf{d}$ given $\mathbf{m}$ (Tarantola, 2005). In many cases, a finite number of parameters, $p$. is determined in order to define a model. In this case, the model parameters can be represented as an $p$-element vector $\mathbf{m}$. Likewise, if there are a finite number of data points then the data can be

expressed as an $q$-element vector $\mathbf{d}$ (note that the use of the integer $m$ for temporal sample number is distinguishable from the model $\mathbf{m}$ by its context). Such problems are called discrete inverse problems or parameter estimation problems. A general parameter estimation problem can be written as Eq. (6.1) (Aster et al., 2005).

The task of estimating $\mathbf{m}$ from $\mathbf{d}$ is called a continuous inverse problem when the model and data are functions of time and space. Normally, problems with small numbers of parameters are referred to as "parameter estimation problems" and problems with a larger number of parameters will be known as "inverse problems" (Aster et al., 2005).

\subsubsection{Function (Operator) G}

When $\mathbf{m}$ and $\mathbf{d}$ are functions, $\mathbf{G}$ is typically referred to an operator. However, $\mathbf{G}$ will be called a function when $\mathbf{m}$ and $\mathbf{d}$ are vectors. The operator $\mathbf{G}$ can take on many forms such as, an ordinary differential equation (ODE) or partial differential equation (PDE). In other cases, $\mathrm{G}$ is a linear or nonlinear system of algebraic equations. 


\subsubsection{Linear versus Non-linear Systems}

Mathematical model of $\mathbf{G}(\mathbf{m})=\mathbf{d}$ could be either linear or non-linear system of equations. Linear systems are those which obey superposition as follows (Proakis \& Manolakis, 2007)

$$
\mathbf{G}\left(c_{1} \mathbf{m}_{1}+c_{2} \mathbf{m}_{2}\right)=c_{1} \mathbf{G}\left(\mathbf{m}_{1}\right)+c_{2} \mathbf{G}\left(\mathbf{m}_{2}\right)
$$

If does not obey the superposition property, the system of equations will be considered nonlinear. Nonlinear mathematical models are defined when desired parameters have an underlying nonlinear relationship to the observable data. The primary nonlinear in this thesis is the phase.

\subsubsection{Overdetermined and Underdetermined Systems of Equations}

If there are more data points than model parameters in the mathematical model $(q>p)$, we will have an over-determined system while if the number of model parameters is greater than data points $(p>q)$, the system will become underdetermined. In each of these two cases, finding a model $\mathbf{m}$ that satisfies every equation exactly will be impossible (Lionheart, 2011). Approximate solutions to such systems are obtainable by solving for model parameters that satisfy the data in a "best fit" sense. In order to find the "best" approximate solution to an inconsistent system of equations, it is tried to find an $\mathbf{m}$ that results in a minimum misfit, or residual, between the data and the forward problem. A usual strategy is to find the model that minimizes the 2 -norm (or Euclidean length) of the residual (Aster et al., 2005)

$$
\|\mathbf{d}-\mathbf{G}(\mathbf{m})\|_{2}=\sqrt{\sum_{i=1}^{m}\left(d_{i}-\mathbf{G}(\mathbf{m})_{i}\right)^{2}}
$$


The model that minimizes the 2-norm is called 2-norm solution or least square solution since it minimizes the sum of the squares of the residuals. The least squares or 2-norm solution is of special interest because it is statistically the most likely solution if data errors are normally distributed (Huffel \& Vandewalle, 1991).

\subsubsection{Statistical Aspects of Least Squares Solution}

If imperfect data points including random errors are given, then a solution aimed to be estimated should be best from statistical point of view. Maximum likelihood (ML) estimation approach seeks a mathematical model $(\mathbf{m})$ that would most likely arise from the observations $(\mathbf{d})$ which have know statistical characteristics. ML method is a general method that can be applied to the estimation problem where a joint probability density function can be assigned to the observations. The observations are assumed to be independent so that the product form of the joint probability density function can be used. Thus, given a model $\mathbf{m}$, the probability density function for each of the $i$ observations will be $f_{l}\left(d_{l} \mid \mathbf{m}\right)$. In general, these probability density functions will vary depending on $\mathbf{m}$ causing the probability densities to be conditional on $\mathbf{m}$. The joint probability density for a vector of independent observations $d$ will be

$$
f(\mathbf{d} \mid \mathbf{m})=f_{1}\left(d_{1} \mid \mathbf{m}\right) \cdot f_{2}\left(d_{2} \mid \mathbf{m}\right) \cdots f_{m}\left(d_{m} \mid \mathbf{m}\right)
$$

The likelihood function is defined as

$$
\begin{aligned}
\mathbf{L}(\mathbf{m} \mid \mathbf{d}) & =f(\mathbf{d} \mid \mathbf{m}) \\
& =f_{1}\left(d_{1} \mid \mathbf{m}\right) \cdot f_{2}\left(d_{2} \mid \mathbf{m}\right) \cdots f_{m}\left(d_{m} \mid \mathbf{m}\right)
\end{aligned}
$$


According to the maximum likelihood principle, model $\mathbf{m}$ that maximizes the likelihood function (Eq. (6.6)) is sought. It is interesting that when the data errors are independent and normally distributed, then the maximum likelihood solution is the least squares solution (Aster et al., 2005). In the following this equivalence is shown.

Assume that the data have independent random errors that are normally distributed with expected value zero, and where the standard deviation of the $i^{t h}$ observation, $d_{\imath}$ is $\sigma_{\imath}$. The probability density for $d_{2}$ then takes the form of

$$
f_{l}\left(d_{\imath} \mid \mathbf{m}\right)=\frac{1}{(2 \pi)^{\frac{1}{2}} \sigma_{l}} \exp \left(-\frac{\left(d_{\imath}-(\mathbf{G}(\mathbf{m}))_{\imath}\right)^{2}}{2 \sigma_{l}^{2}}\right)
$$

The likelihood function for the complete data set is the product of the individual likelihoods

$$
\mathbf{L}(\mathbf{m} \mid \mathbf{d})=\frac{1}{(2 \pi)^{\frac{m}{2}} \prod_{\imath=1}^{m} \sigma_{\iota}} \prod_{\imath=1}^{m} \exp \left(-\frac{\left(d_{\iota}-(\mathbf{G}(\mathbf{m}))_{\iota}\right)^{2}}{2 \sigma_{\imath}^{2}}\right)
$$

By doing some calculations, it will be found out that the maximum of Eq. (6.8) is equivalent to

$$
\min _{\mathbf{m}} \sum_{\imath=1}^{m} \frac{\left(d_{\imath}-(\mathbf{G}(\mathbf{m}))_{\imath}\right)^{2}}{\sigma_{\imath}^{2}}
$$

Or equivalently

$$
\min _{\mathbf{m}} \sqrt{\sum_{\imath=1}^{m} \frac{\left(d_{\imath}-(\mathbf{G}(\mathbf{m}))_{\imath}\right)^{2}}{\sigma_{\imath}^{2}}}
$$


It can be seen from equations (6.10) and (6.4) that the maximum likelihood solution is the same as the least square solution. The only change is that the standard deviation of noise is also considered in this last equation (Aster et al., 2005).

\subsubsection{Ill-conditioning}

One characteristic of many (discrete) inverse problems is that they are ill-conditioned. That is, the process of finding an inverse solution is highly unstable in that a small change in measurement, e.g., a small noise in Eq. (6.2), can result in a vast change in the estimated model. Continuous and discrete inverse problems where this situation happens are referred to as illposed and ill-conditioned, respectively (Aster et al., 2005).

It is possible to stabilize the inversion process by regularization techniques. In these techniques, additional constraints that bias the solution are imposed in a way that a usable solution can be obtained (Aster et al., 2005).

\subsubsection{Selecting a Good Solution by Regularization}

For a general least squares problem there may be infinitely many least squares solutions. If noise is included in the data, and that there is no point in fitting such noise exactly, apparently there might be many solutions sufficiently fit the data in the way that $\|\mathbf{G}(\mathbf{m})-\mathbf{d}\| \leq \delta$ is small enough. Normally, all solutions with $\|\mathbf{G}(\mathbf{m})-\mathbf{d}\| \leq \delta$. are considered and among these solutions the one which minimizes the norm of $\mathbf{m}$ as follows is selected (Aster et al., 2005).

$$
\begin{gathered}
\min \|\mathbf{m}\|_{2} \\
\text { such that }\|\mathbf{G}(\mathbf{m})-\mathbf{d}\|_{2} \leq \delta
\end{gathered}
$$


In order to fit the data, nonzero features are required to appear in the solution. Each nonzero feature will increase the norm of $\mathbf{m}$. Therefore, minimization of $\|\mathbf{m}\|_{2}$ will ensure that unneeded features will not appear in the regularized solution. As $\delta$ increases, the set of possible models enlarged while the minimum value of $\|\mathbf{m}\|_{2}$ decreased. Thus, it is possible to trace out a curve of minimum values of $\|\mathbf{m}\|_{2}$ versus $\delta$ (Figure 6.1).

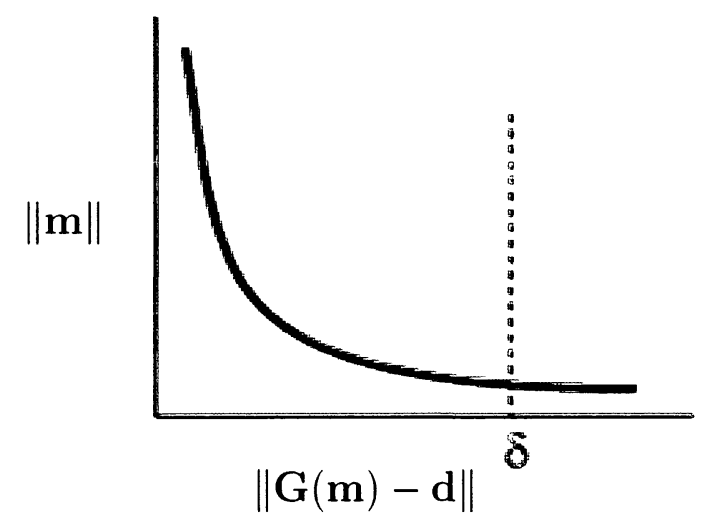

Figure 6.1 Curve of the misfit norms and associated model norms

An alternative option is to consider the damped least squares problems using Tikhonov regularization (Engl et al., 2005; Aster et al., 2005) as follows

$$
\min \|\mathbf{G}(\mathbf{m})-\mathbf{d}\|_{2}^{2}+\mu^{2}\|\mathbf{m}\|_{2}^{2}
$$

which is resulted when the method of Lagrange multipliers is applied to (6.11). The parameter $\mu$ represented in Eq. (6.12) is known as the regularization parameter. For proper choices of $\delta$ and $\mu$, the two problems of (6.11) and (6.12) return the same solution. In this work, the focus is on 
solving the damped least squares form of the problem shown in Eq. (6.12). In these problems, it is desired to find $\mu$ that leads to the best solution. Later on in this chapter, it will be explained how $\mu$ can be selected.

\subsection{Higher-Order Tikhonov Regularization}

In Tikhonov regularization explained above, an objective function involving $\|\mathbf{m}\|_{2}$ was intended to be minimized. Most of the time, it is preferred to find a solution that minimizes some other measure of $\mathbf{m}$, such as the norm of the first or second derivative (Aster et al., 2005). The discrete first derivative of the model can be calculated by $\Gamma \mathrm{m}$, where

$$
\boldsymbol{\Gamma}=\left[\begin{array}{ccccc}
-1 & 1 & & & \\
& -1 & 1 & & \\
& & \cdots & & \\
& & -1 & 1 & \\
& & & -1 & 1
\end{array}\right]
$$

Matrices that are used to differentiate $\mathbf{m}$ for the purpose of regularization are referred to as roughening matrices. In (6.13), $\Gamma \mathrm{m}$ is a finite-difference approximation that is proportional to the first derivative of $\mathbf{m}$. By minimizing $\|\Gamma \mathrm{m}\|_{2}$, relatively flat solutions are desired (Engl \& Kügler, 2005). In first-order Tikhonov regularization, we solve the damped least squares problem of

$$
\min \|\mathbf{G}(\mathbf{m})-\mathbf{d}\|_{2}^{2}+\mu^{2}\|\mathbf{\Gamma} \mathbf{m}\|_{2}^{2}
$$

where $\Gamma$ is the matrix represented in (6.13). 
In second-order Tikhonov regularization,

$$
\boldsymbol{\Gamma}=\left[\begin{array}{ccccccc}
1 & -2 & 1 & & & & \\
& 1 & -2 & 1 & & & \\
& & & \cdots & & & \\
& & & 1 & -2 & 1 & \\
& & & & 1 & -2 & 1
\end{array}\right]
$$

is used. Here, $\Gamma \mathrm{m}$ is a finite-difference approximation proportional to the second derivative of m. By minimizing $\|\Gamma \mathrm{m}\|_{2}$, those solutions that are not smooth in a second derivative sense are penalized (Engl et al., 2005; Aster et al., 2005).

So far explanations about inverse problems are general and can be applied to both linear and nonlinear inverse problems. The inverse problem defined in this research has a nonlinear representation. Therefore, details of linear inverse problems and associated techniques for solving them are left here.

Common approaches to solving nonlinear inverse problems are introduced in the next section. First, Newton's method will be described as a general framework for solving nonlinear systems of equations. Then, a discussion will be provided about the Gauss-Newton (GN) method which is a version of Newton's method designed for nonlinear inverse problems.

\subsection{Nonlinear Regression}

A nonlinear system of equations is defined as 


$$
\mathbf{F}(\mathbf{x})=\left[\begin{array}{c}
f_{1}(\mathbf{x}) \\
f_{2}(\mathbf{x}) \\
\vdots \\
f_{p}(\mathbf{x})
\end{array}\right]
$$

where $\mathbf{x}=\left(x_{1}, x_{2}, \cdots, x_{p}\right)$ refers to $p$ variables, and $f_{1}, \cdots, f_{p}$ are nonlinear functions (equations). Finding a solution for a nonlinear system of equations $f(\mathbf{x})$ involves finding a solution which satisfies every equation of the system (Grosan \& Abraham, 2008).

In general, the problem of finding a parameterized curve (or a solution for the system) that approximately fits a set of data is referred to as regression. When the regression model is nonlinear in the fitted parameters, the problem will become a nonlinear regression.

\subsubsection{Newton's Method}

\subsubsection{Finding the Root of a Nonlinear System}

Consider a nonlinear system of $p$ equations in $p$ unknowns

$$
\mathbf{F}(\mathbf{x})=0
$$

In order to obtain a solution for Eq. (6.17), a sequence of vectors, $\mathbf{x}^{0}, \mathbf{x}^{1}, \cdots$, which will converge to a solution $\mathbf{x}^{*}$, will be constructed. Then, if $\mathbf{F}$ is continuously differentiable, a first order Taylor expansion about $\mathbf{x}^{0}$ is constructed (Grosan et al., 2008; Aster et al., 2005) as

$$
\mathbf{F}\left(\mathbf{x}^{0}+\Delta \mathbf{x}\right) \approx \mathbf{F}\left(\mathbf{x}^{0}\right)+\nabla \mathbf{F}\left(\mathbf{x}^{0}\right) \Delta \mathbf{x}
$$

where $\nabla \mathbf{F}\left(\mathbf{x}^{0}\right)$ is the Jacobean defined as 


$$
\nabla \mathbf{F}\left(\mathbf{x}^{0}\right)=\left[\begin{array}{ccc}
\frac{\partial F_{1}\left(\mathbf{x}^{0}\right)}{\partial x_{1}} & \cdots & \frac{\partial F_{1}\left(\mathbf{x}^{0}\right)}{\partial x_{p}} \\
\vdots & \ddots & \vdots \\
\frac{\partial F_{p}\left(\mathbf{x}^{0}\right)}{\partial x_{1}} & \cdots & \frac{\partial F_{p}\left(\mathbf{x}^{p}\right)}{\partial x_{1}}
\end{array}\right]
$$

Using Eq. (6.18), an approximate equation for the difference between $x^{0}$ and the unknown $x^{*}$ can be obtained. Setting the right side of the Eq. (6.18) to zero leads to

$$
\mathbf{F}\left(\mathbf{x}^{*}\right)=0 \approx \mathbf{F}\left(\mathbf{x}^{0}\right)+\nabla \mathbf{F}\left(\mathbf{x}^{0}\right) \Delta \mathbf{x}
$$

By solving Eq. (6.20) for the difference between $\mathbf{x}^{*}$ and $\mathbf{x}^{0}$

$$
\nabla \mathbf{F}\left(\mathbf{x}^{0}\right) \Delta \mathbf{x} \approx-\mathbf{F}\left(\mathbf{x}^{0}\right)
$$

will be resulted which leads to Newton's method algorithm summarized as follows

\section{Algorithm for Newton's Method}

Given a system of equations $\mathbf{F}(\mathbf{x})=0$, set $\mathbf{k}=0$ and guess an initial solution $\mathbf{x}^{0}$, then

\section{Repeat}

1. Compute $\nabla \mathbf{F}\left(\mathbf{x}^{k}\right)$ and $\mathbf{F}\left(\mathbf{x}^{k}\right)$

2. Use Gaussian elimination to solve $\nabla \mathbf{F}\left(\mathbf{x}^{k}\right) \Delta \mathbf{x}=-\mathbf{F}\left(\mathbf{x}^{k}\right)$

3. Let $\mathbf{x}_{k+1}=\mathbf{x}_{k}+\Delta \mathbf{x}$

\section{Let $\mathbf{k}=\mathbf{k}+1$}

Until the sequence converges to a solution with $\mathbf{F}(\mathbf{x})$ sufficiently close to 0 (Grosan \& Abraham, 2008). 
If $\mathbf{x}^{0}$ is sufficiently close to $\mathbf{x}^{*}, \mathbf{F}\left(\mathbf{x}^{*}\right)$ will be continuously differentiable in a neighborhood of $\mathbf{x}^{*}$ and Newton's method will converge to $\mathbf{x}^{*}$. Otherwise, the Newton's method will either converge slowly or fail (Aster et al., 2005).

\subsubsection{Finding the Minimum of a Nonlinear System}

Now, if it is desired to find the minimum of a function $f(\mathbf{x})$ which is assumed to be twice continuously differentiable, then the Newton's method can be used in a similar way to find the local minimum of function $f(\mathbf{x})$ (Aster et al., 2005). The Taylor series expansion of $f(\mathbf{x})$ about $\mathbf{x}^{0}$ can be written as

$$
f\left(\mathbf{x}^{0}+\Delta \mathbf{x}\right) \approx f\left(\mathbf{x}^{0}\right)+\nabla f\left(\mathbf{x}^{0}\right)^{T} \Delta \mathbf{x}+\frac{1}{2} \Delta \mathbf{x}^{T} \nabla^{2} f\left(\mathbf{x}^{0}\right) \Delta \mathbf{x}
$$

where $\nabla f\left(\mathbf{x}^{0}\right)$ is the gradient

$$
\nabla f\left(\mathbf{x}^{0}\right)=\left[\begin{array}{c}
\frac{\partial f\left(\mathbf{x}^{0}\right)}{\partial x_{1}} \\
\vdots \\
\frac{\partial f\left(\mathbf{x}^{0}\right)}{\partial x_{p}}
\end{array}\right]
$$

and $\nabla^{2} f\left(\mathbf{x}^{0}\right)$ is the Hessian

$$
\nabla^{2} f\left(\mathbf{x}^{0}\right)=\left[\begin{array}{ccc}
\frac{\partial^{2} f\left(\mathbf{x}^{0}\right)}{\partial x_{1}^{2}} & \cdots & \frac{\partial^{2} f\left(\mathbf{x}^{0}\right)}{\partial x_{1} \partial x_{p}} \\
\vdots & \ddots & \vdots \\
\frac{\partial^{2} f\left(\mathbf{x}^{0}\right)}{\partial x_{p} \partial x_{1}} & \cdots & \frac{\partial^{2} f\left(\mathbf{x}^{0}\right)}{\partial x_{p}^{2}}
\end{array}\right]
$$


$\nabla^{2}$ is used to denote the Hessian. A necessary condition for $\mathbf{x}^{*}$ to be a minimum of $f(\mathbf{x})$ is that $\nabla f\left(\mathbf{x}^{*}\right)=0$. The gradient can be approximated in the vicinity of $\mathbf{x}^{0}$ by (simply from Eq. (6.18))

$$
\nabla f\left(\mathbf{x}^{0}+\Delta \mathbf{x}\right) \approx \nabla f\left(\mathbf{x}^{0}\right)+\nabla^{2} f\left(\mathbf{x}^{0}\right) \Delta \mathbf{x}
$$

Setting the approximate gradient of Eq. (6.26) equal to zero gives

$$
\nabla^{2} f\left(\mathbf{x}^{0}\right) \Delta \mathbf{x} \approx-\nabla f\left(\mathbf{x}^{0}\right)
$$

Solving Eq. (6.27) for successive solution steps leads to Newton's method algorithm for minimizing $f(\mathbf{x})$. Newton's method used to minimize the function $f(\mathbf{x})$ is equivalent to the Newton's method applied to a nonlinear system of equations of $\nabla f(\mathbf{x})=0$.

\subsubsection{Gauss-Newton Method}

Newton's method for systems of equations is not directly applicable to most nonlinear inverse problems. As mentioned before, this is because the number of data points and model parameters may not be equal and thus, an exact solution to $G(m)=d$ may not be existed. In these conditions, the Newton's method will be applied to minimize a nonlinear least squares problem. Specifically, the problem of fitting a vector of $q$ parameters to a data vector $\mathbf{d}$ will be considered.

The parameters and data are related by a mathematical model of $\mathbf{G}(\mathbf{m})=\mathbf{d}$. A vector of standard deviations $\sigma$ for the data measurements is also determined. The goal is to find values of the parameters that best fit the data in the sense of minimizing the 2 -norm of the residuals. If we assume that the measurement errors are normally distributed, then the maximum likelihood 
principle leads us to minimizing the sum of squared errors normalized by their respective standard deviations (Aster et al., 2005). We seek to minimize

$$
f(\mathbf{m})=\sum_{i=1}^{q}\left(\frac{G(m)_{\imath}-d_{\imath}}{\sigma_{\imath}}\right)^{2}
$$

For convenience, we will let

$$
f_{i}(\mathbf{m})=\frac{G(\mathbf{m})_{,}-d_{l}}{\sigma_{l}} . \quad \imath=1.2 . \cdots . q
$$

and

$$
\mathbf{F}(\mathbf{m})=\left[\begin{array}{c}
f_{1}(\mathbf{m}) \\
\vdots \\
f_{q}(\mathbf{m})
\end{array}\right]
$$

Thus,

$$
f(\mathbf{m})=\sum_{i=1}^{q} f_{l}(\mathbf{m})^{2}
$$

The gradient of $f(\mathbf{m})$ can be written in matrix notation as

$$
\nabla f(\mathbf{m})=2 \mathbf{J}(\mathbf{m})^{T} \mathbf{F}(\mathbf{m})
$$


where $\mathbf{J}(\mathbf{m})$ is the Jacobean.

$$
\mathbf{J}(\mathbf{m})=\nabla \mathbf{F}\left(\mathbf{x}^{0}\right)=\left[\begin{array}{ccc}
\frac{\partial f_{1}(\mathbf{m})}{\partial m_{1}} & \cdots & \frac{\partial f_{1}(\mathbf{m})}{\partial m_{p}} \\
\vdots & \ddots & \vdots \\
\frac{\partial f_{y}(\mathbf{m})}{\partial m_{1}} & \cdots & \frac{\partial f_{q}(\mathbf{m})}{\partial m_{p}}
\end{array}\right]
$$

Similarly, we can express the Hessian of $f(\mathbf{m})$ as

$$
\nabla^{2} f(\mathbf{m})=2 \mathbf{J}(\mathbf{m})^{T} \mathbf{J}(\mathbf{m})+2 \sum_{\imath=1}^{q} f_{\imath}(\mathbf{m}) \nabla^{2} f_{\imath}(\mathbf{m})
$$

In the Gauss-Newton (GN) method, the second added term is ignored and the Hessian is approximated by the first term of Eq. (6.34) as

$$
\nabla^{2} f(\mathbf{m}) \approx 2 \mathbf{J}(\mathbf{m})^{T} \mathbf{J}(\mathbf{m})
$$

Using Eq. (6.32) and (6.35) and dividing both sides by 2, the equations for successive iteration in the GN method become

$$
\mathbf{J}\left(\mathbf{m}^{k}\right)^{T} \mathbf{J}\left(\mathbf{m}^{k}\right) \Delta \mathbf{m}=-\mathbf{J}\left(\mathbf{m}^{k}\right)^{T} \mathbf{F}\left(\mathbf{m}^{k}\right)
$$

To regularize the solution obtained by GN method and find a solution with smallest $\|\mathbf{m}\|_{2}$, Tikhonov regularization is used. Therefore, the nonlinear least squares problem is reformulated as a damped least squares problem as follow (Aster et al., 2005) 


$$
\min \|\mathbf{G}(\mathbf{m})-\mathbf{d}\|_{2}^{2}+\mu^{2}\|\mathbf{\Gamma} \mathbf{m}\|_{2}^{2}
$$

As explained before, the regularization is done to avoid ill-conditioning. The regularized least squares problem can be solved by applying the GN method to it. To apply the GN method to Eq. (6.37), it is rewritten as (Aster et al., 2005)

$$
\min \|\underset{\mu \mathbf{\Gamma m}}{\mathbf{G}(\mathbf{m})-\mathbf{d}}\|_{2}^{2}
$$

The Jacobean for this damped least squares problem for the $k^{\text {th }}$ iteration is

$$
\mathbf{K}\left(\mathbf{m}^{k}\right)=\left[\begin{array}{c}
\mathbf{J}\left(\mathbf{m}^{k}\right) \\
\mu \boldsymbol{\Gamma}
\end{array}\right]
$$

where $\mathbf{J}\left(\mathbf{m}^{k}\right)$ is the Jacobean of $\mathbf{G}\left(\mathbf{m}^{k}\right)$. A GN method step is obtained by solving

$$
\mathbf{K}\left(\mathbf{m}^{k}\right)^{T} \mathbf{K}\left(\mathbf{m}^{k}\right) \Delta \mathbf{m}=-\mathbf{K}\left(\mathbf{m}^{k}\right)^{T}\left[\begin{array}{c}
\mathbf{G}\left(\mathbf{m}^{k}\right)-\mathbf{d} \\
\mu \boldsymbol{\Gamma} \mathbf{m}^{k}
\end{array}\right]
$$

It can be simplified using Eq. (6.39) to (Jin, 2008; Aster et al., 2005)

$$
\left(\mathbf{J}\left(\mathbf{m}^{k}\right)^{T} \mathbf{J}\left(\mathbf{m}^{k}\right)+\mu^{2} \boldsymbol{\Gamma}^{T} \boldsymbol{\Gamma}\right) \Delta \mathbf{m}=-\mathbf{J}\left(\mathbf{m}^{k}\right)^{T}\left(\mathbf{G}\left(\mathbf{m}^{k}\right)-\mathbf{d}\right)-\mu^{2} \boldsymbol{\Gamma}^{T} \boldsymbol{\Gamma} \mathbf{m}^{k}
$$

The GN algorithm will find a minimum of the function at which the solution gradient is zero. Unfortunately, the minimum value found by the GN algorithm may not be the global minimum, 
but simply a local minimum need the iteration starting point. This problem becomes more severe as the objective function is more non-linear.

\subsection{Defined Inverse Problem for this Research}

\subsubsection{Inverse Problem Determination}

As explained in the beginning of this chapter, it is desired to improve the phase (phase shift) information obtained by the quadrature method making them closer to the real phase (phase shift) information resulted from the reference (phasor) method. It is also desired to fix phase jumps appeared in the phase (phase shift) results, especially those which are less than $2 \pi$ since these phase jumps cannot be fixed by unwrap process. Inverse problem techniques seem to be able to solve these problems to some extent.

If the real phase information is considered as a model and the results obtained via quadrature method are considered as observable data, then we can define a specific inverse problem in which the model can be estimated.

In chapter 5, the phase shift occurred between the first two RF signals was examined in different conditions. Similarly, in this chapter the inverse problem is defined for the first two RF signals. The procedure however, can be generalized for other RF signals with some modifications. Note that, at this step the noise removal is aimed. Later on when the phase jump reconstruction is pursued, another RF signal pairs will be used.

For the first RF signal, two forward models were defined separately as

$$
\left\{\begin{array}{l}
\mathbf{G}_{1}(\mathbf{m})=\mathbf{d}_{1} \\
\mathbf{G}_{2}(\mathbf{m})=\mathbf{d}_{2}
\end{array}\right.
$$


The observable data $\mathbf{d}_{\mathbf{1}}$ and $\mathbf{d}_{\mathbf{2}}$ were defined as the in-phase and quadrature parts of the complex baseband signal obtained via the quadrature method, respectively. As mentioned in chapter 4 , the complex baseband signal has the representation of

$$
u(n)=I(n)+j Q(n)
$$

where $I(n)$ and $Q(n)$ are the in-phase and quadrature parts with representations of

$$
\begin{array}{cc}
I(n)=\sum_{k=1}^{L} \frac{A_{k}(n)}{2} \cos \left[\Delta \omega n T_{s}-\phi_{k}(n)\right] \quad n=1: N \\
Q(n)=\sum_{k=1}^{L} \frac{A_{k \cdot}(n)}{2} \sin \left[\Delta \omega n T_{s}-\phi_{k}(n)\right] \quad n=1: N
\end{array}
$$

Here $n$ and $L$ are depth sample number and the number of scatterers, respectively, and $N$ is the maximum depth sample number. Data $\mathbf{d}_{\mathbf{1}}$ and $\mathbf{d}_{\mathbf{2}}$ were defined as $q$-element vectors of

$$
\mathbf{d}_{1}=\left[\begin{array}{c}
d_{1} \\
d_{2} \\
\vdots \\
d_{N}
\end{array}\right]_{q \times 1}
$$

and

$$
\mathbf{d}_{2}=\left[\begin{array}{c}
d_{1}^{\prime} \\
d_{2}^{\prime} \\
\vdots \\
d_{N}^{\prime}
\end{array}\right]_{q \times 1}
$$


with $d_{n}$ and $d_{n}^{\prime}$ defined as

$$
\begin{aligned}
& d_{n}=I(n)=\sum_{k=1}^{L} \frac{A_{k}(n)}{2} \cos \left(-\phi_{k}(n)\right) . \quad n=1: N \\
& d_{n}^{\prime}=Q(n)=\sum_{k=1}^{L} \frac{A_{k}(n)}{2} \sin \left(-\phi_{k}(n)\right) . \quad n=1: N
\end{aligned}
$$

where $n$ is depth sample number which starts from 1 to $N$ and $q$ which represents the number of data points is equal to $N$. The model (parameters) to be estimated was defined as an $p$-element vector $\mathbf{m}$ as

$$
\mathbf{m}=\left[\begin{array}{c}
\varphi(1) \\
\varphi(2) \\
\vdots \\
\varphi(N) \\
\mathrm{A}(1) \\
\mathrm{A}(2) \\
\vdots \\
\mathrm{A}(N)
\end{array}\right]_{p \times 1}
$$

Here, $p$. the number of parameters in vector $\mathbf{m}$ is equal to $2 N$ and $\varphi(n)$ and $\mathrm{A}(n)$ are the phase and amplitudes of the received RF signal at different depth sample numbers, respectively. The first $N$ elements of the model vector are the phase parameters which are desired to be estimated in this research.

With data and model definitions, systems of $\mathbf{G}_{1}(\mathbf{m})$ and $\mathbf{G}_{2}(\mathbf{m})$ were defined as 


$$
\mathbf{G}_{1}(\mathbf{m})=\left[\begin{array}{c}
g_{1}(\mathbf{m}) \\
g_{2}(\mathbf{m}) \\
\vdots \\
g_{q}(\mathbf{m})
\end{array}\right]=\left[\begin{array}{c}
\mathrm{A}(1) \cos (\varphi(1)) \\
\mathrm{A}(2) \cos (\varphi(2)) \\
\vdots \\
\mathrm{A}(N) \cos (\varphi(N))
\end{array}\right]
$$

and

$$
\mathbf{G}_{2}(\mathbf{m})=\left[\begin{array}{c}
g_{1}^{\prime}(\mathbf{m}) \\
g_{2}^{\prime}(\mathbf{m}) \\
\vdots \\
g_{q}^{\prime}(\mathbf{m})
\end{array}\right]=\left[\begin{array}{c}
\mathrm{A}(1) \sin (\varphi(1)) \\
\mathrm{A}(2) \sin (\varphi(2)) \\
\vdots \\
\mathrm{A}(N) \sin (\varphi(N))
\end{array}\right]
$$

\subsubsection{GN Algorithm Application}

Since $\mathbf{d}_{1}$ and $\mathbf{d}_{2}$ are collections of discrete observations and $\mathbf{m}$ is a collection of finite number of parameters, the problem of finding $\mathbf{m}$ given $\mathbf{d}$ in this research is a discrete inverse problem. Besides, defined mathematical models of $\mathbf{G}_{1}(\mathbf{m})=\mathbf{d}_{1}$ and $\mathbf{G}_{1}(\mathbf{m})=\mathbf{d}_{1}$ are nonlinear systems of equations due to the fact that the parameters of interest have a nonlinear relationship to the observables. The number of model parameters in defined inverse problems is greater than data points, and therefore, the systems of equations are underdetermined. As explained before, in the case of having underdetermined system of equations, $\mathbf{m}$ should be estimated by minimizing the 2-norm of misfit between the data and forward model. In other words, defined inverse problems are nonlinear least squares problems which needed to be solved by iterative Gauss-Newton method. In order to apply Gauss-Newton method, the matrix of residuals for the systems was defined as 


$$
\mathbf{F}_{1}(\mathbf{m})=\left[\begin{array}{c}
f_{1}(\mathbf{m}) \\
f_{2}(\mathbf{m}) \\
\vdots \\
f_{q}(\mathbf{m})
\end{array}\right]: \quad f_{\imath}(\mathbf{m})=\frac{g_{l}(\mathbf{m})-d_{l}}{\sigma} . \quad i=1: q
$$

and

$$
\mathbf{F}_{2}(\mathbf{m})=\left[\begin{array}{c}
f_{1}^{\prime}(\mathbf{m}) \\
f_{2}^{\prime}(\mathbf{m}) \\
\vdots \\
f_{q}^{\prime}(\mathbf{m})
\end{array}\right]: \quad f_{1}^{\prime}(\mathbf{m})=\frac{g_{2}^{\prime}(\mathbf{m})-d_{2}^{\prime}}{\sigma}, \quad i=1: q
$$

Similarly, the corresponding Jacobean matrices are defined as

$$
\begin{aligned}
\mathbf{J}_{1}(\mathbf{m}) & =\nabla \mathbf{F}_{1}(\mathbf{m}) \\
& =\left[\begin{array}{cccccccc}
\frac{-\mathrm{A}(1) \sin (\varphi(1))}{\sigma} & 0 & \ldots & 0 & \frac{\cos (\varphi(1))}{\sigma} & \ldots & 0 & 0 \\
0 & \frac{-A(2) \sin (\varphi(2))}{\sigma} & 0 & \ldots & 0 & \frac{\cos (\varphi(2))}{\sigma} & \ldots & 0 \\
\vdots & \vdots & \ddots & \ldots & \ddots & \ldots & \ddots & \vdots \\
0 & 0 & \ldots & \frac{-\mathrm{A}(N) \sin (\varphi(N))}{\sigma} & 0 & \ldots & 0 & \frac{\cos (\varphi(N))}{\sigma}
\end{array}\right]_{q \times 2 q}
\end{aligned}
$$

(6.57)

and 


$$
\mathbf{J}_{2}(\mathbf{m})=\nabla \mathbf{F}_{2}(\mathbf{m})
$$

$$
=\left[\begin{array}{cccccccc}
\frac{\mathrm{A}(1) \cos (\varphi(1))}{\sigma} & 0 & \ldots & 0 & \frac{\sin (\varphi(1))}{\sigma} & \ldots & 0 & 0 \\
0 & \frac{\mathrm{A}(2) \cos (\varphi(2))}{\sigma} & 0 & \ldots & 0 & \frac{\operatorname{in}(\varphi(2))}{\sigma} & \ldots & 0 \\
\vdots & \vdots & \ddots & \ldots & \ddots & \ldots & \ddots & \vdots \\
0 & 0 & \ldots & \frac{\mathrm{A}(N) \cos (\varphi(N))}{\sigma} & 0 & \ldots & 0 & \frac{\sin (\varphi(N))}{\sigma}
\end{array}\right]_{q \times 2 q}
$$

respectively. The $\sigma$ parameter represented in above matrices, is the standard deviation of the noise which can be added to the data. In order to generate noisy data, white Gaussian noise with mean zero and standard deviation of $\sigma$ was added to the observable data $\mathbf{d}_{\mathbf{1}}$ and $\mathbf{d}_{\mathbf{2}}$.

Based on Eq. (6.41), the equations for successive iteration in the GN method becomes equal to

$$
\begin{aligned}
{\left[\mathbf{J}_{1}\left(\mathbf{m}^{k}\right)^{T} \mathbf{J}_{1}\left(\mathbf{m}^{k}\right)+\mathbf{J}_{2}\left(\mathbf{m}^{k}\right)^{T} \mathbf{J}_{2}\left(\mathbf{m}^{k}\right)+\mu^{2} \boldsymbol{\Gamma}^{T} \boldsymbol{\Gamma}\right] \Delta \mathbf{m} } & \\
& =\left[-\mathbf{J}_{1}\left(\mathbf{m}^{k}\right)^{T} \mathbf{F}_{1}\left(\mathbf{m}^{k}\right)\right]+\left[-\mathbf{J}_{2}\left(\mathbf{m}^{k}\right)^{T} \mathbf{F}_{2}\left(\mathbf{m}^{k}\right)\right]-\mu^{2} \boldsymbol{\Gamma}^{T} \boldsymbol{\Gamma} \mathbf{m}^{k}
\end{aligned}
$$

The presence of regularization parameter $(\mu)$ and roughening matrix $(\boldsymbol{\Gamma})$ in Eq. $(6.59)$ is to avoid ill-conditioning and to obtain a regularized solution.

\subsubsection{Reconstructed Phase (Phase Shift) Results via the GN Algorithm}

With different SNRs, different phase and phase shift plots obtained via the quadrature technique were reconstructed by defined GN algorithms. As an example, phase information of the second RF signal with the SNR of $10 \mathrm{~dB}$ is shown in Figure 6.2. The phase shift between the first two 
(pre- and post-compression) RF signals is represented in Figure 6.3. These plots are resulted from three different methods of phasor (reference), quadrature, and GN algorithm and represented in three different colours.
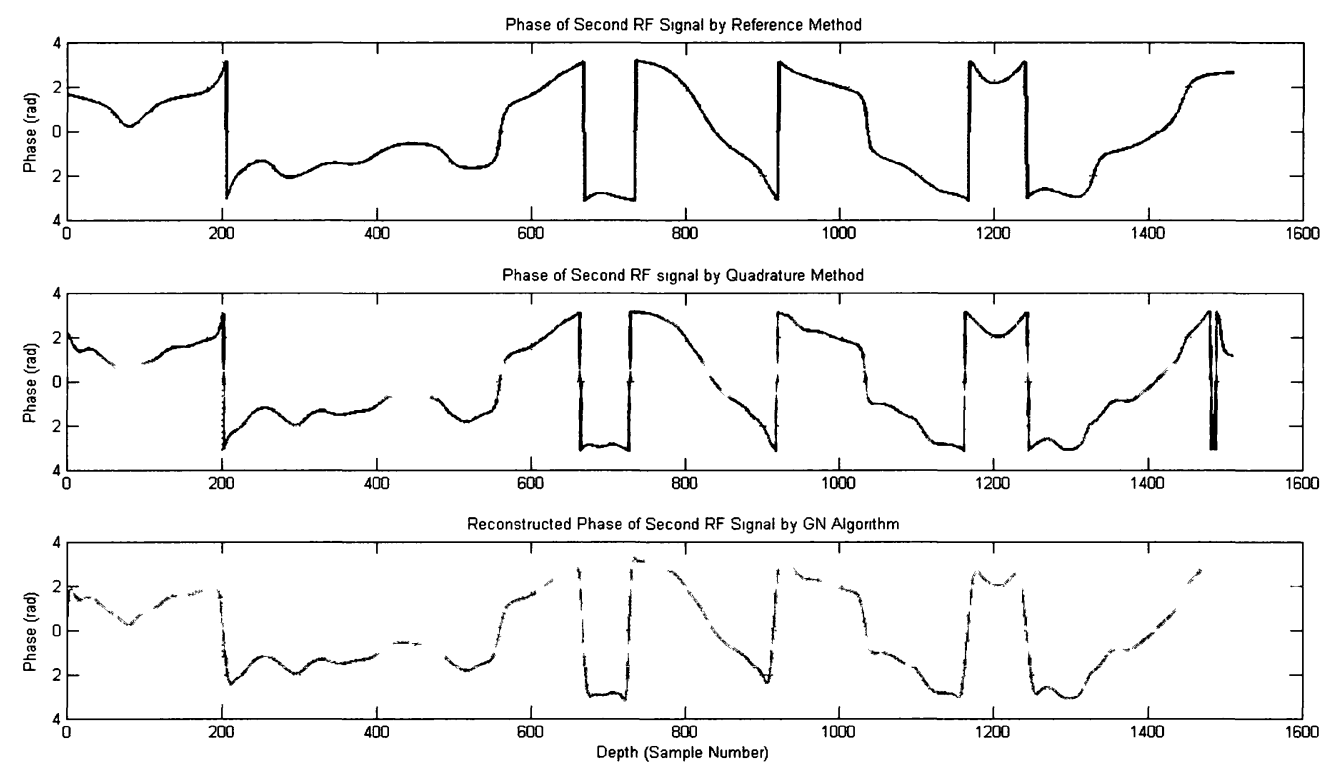

Figure 6.2 Phase of the second (post-compression) RF signal $(\mathrm{SNR}=10 \mathrm{~dB})$. Phase obtained via the reference method (top), phase resulted from the quadrature method (middle), the reconstructed phase obtained by the GN algorithm (bottom). 

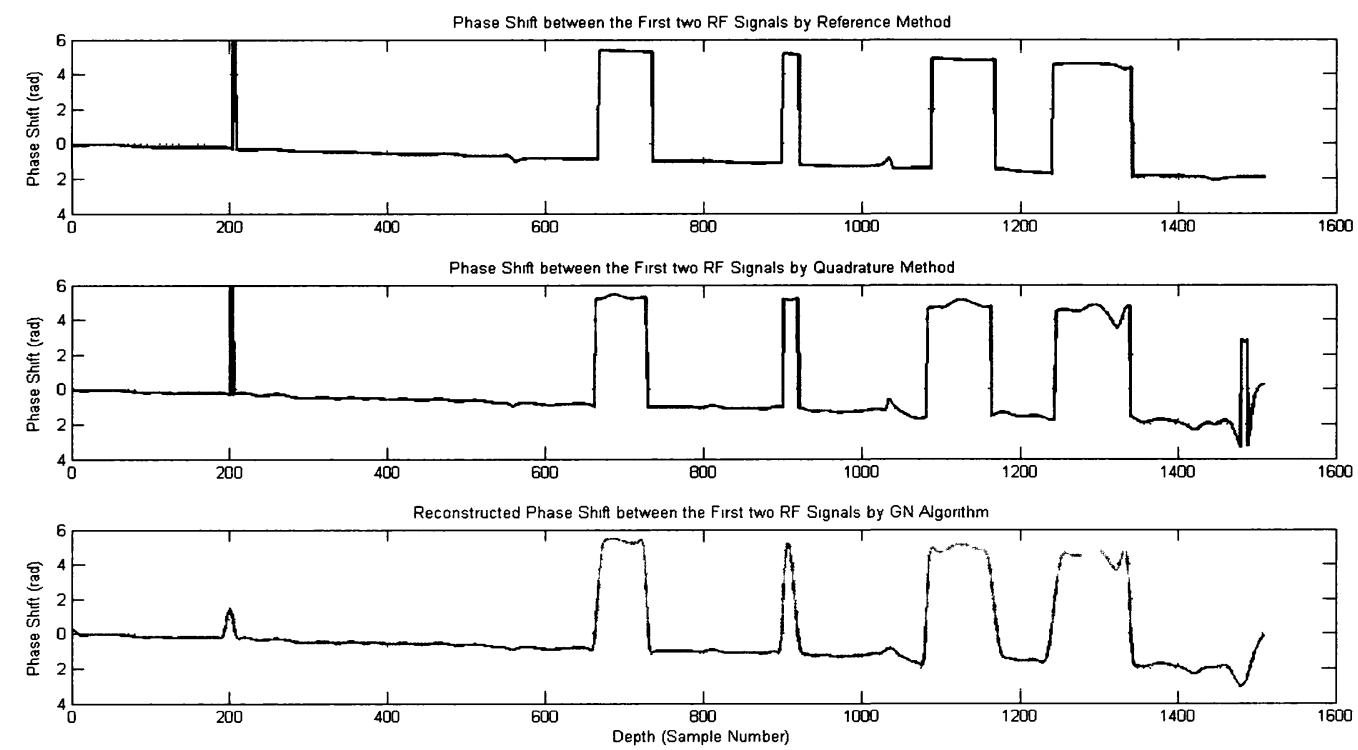

Figure 6.3 Phase shift between the first two (pre- and post-compression) RF signal (SNR $=10 \mathrm{~dB}$ ). Phase shift obtained via the reference method (top), phase shift resulted from the quadrature method (middle), the reconstructed phase shift obtained by the GN algorithm application (bottom). Regularization parameters of the applied GN algorithms are optimal.

In application of the GN algorithm and based on Eq. (6.57), regularization parameter $(\mu)$ and roughening matrix $(\Gamma)$ should be determined. In this research, second-order Tikhonov regularization was used since it resulted in better outcomes compare to other types of Tikhonov regularization.

During the simulation, the optimal regularization parameter $(\mu)$ and the number of iterations were varied based on the amount of noise added. For the second RF signal with the SNR of 10 $\mathrm{dB}$, the optimal regularization parameter and the number of iterations were found to be 14 and 7 , respectively. These optimal values were obtained in this way: the reconstructed phase error (misfit between the reconstructed phase and the real phase), shown by $\varepsilon_{2}$, was plotted with respect to different regularization parameters and iterations number. Then, by tracing out the resulted plots (Figure 6.4), those regularization parameters and numbers of iteration which 
resulted in the minimum $\varepsilon_{2}$ were selected. In Figure 6.4, reconstructed phase errors $\left(\varepsilon_{2}\right)$ versus regularization parameter $(\mu)$ are shown. Different colors show different number of iterations used. The dashed line in this figure is the quadrature phase error (misfit between noisy phase measured by the quadrature method and the real phase) which is shown by $\varepsilon_{1}$. If the regularization parameter is selected from the regions where $\varepsilon_{2}$ is under the dashed line, then it means that the employed GN algorithm can improve the noisy phase making it closer to the real phase. Thses regions are considered as the accepted regions from which the regularization parameter(s) can be chosen.

To measure the norm of errors $\varepsilon_{1}$ and $\varepsilon_{2}$, the following approach was taken: the sample numbers spanning the time interval of the phase signals were divided in a number of intervals with the end points at samples that $2 \pi$ jumps occure. The norm of errors was calculated at each interval and then averaged. The averaged error norm was then plotted (Figure 6.4).

Sample divisions were made to cancel the effect of jumps in calculating the error norms. If samples were not divided into sub intervals, the GN reconstructive algorithm, which smoothen the curve around the spikes (jumps), would result in a larger $\left\|\varepsilon_{2}\right\|$. It is due to the fact that the $\varepsilon_{2}$ is calculated with respect to the phase resulted from the reference method and not with the actual phase which is not wrapped and doesn't have jumps at all. That is, if the $\left\|\varepsilon_{2}\right\|$ is found to be large (with respect to the reference phase), it can in fact be small if compared with the actual phase. 


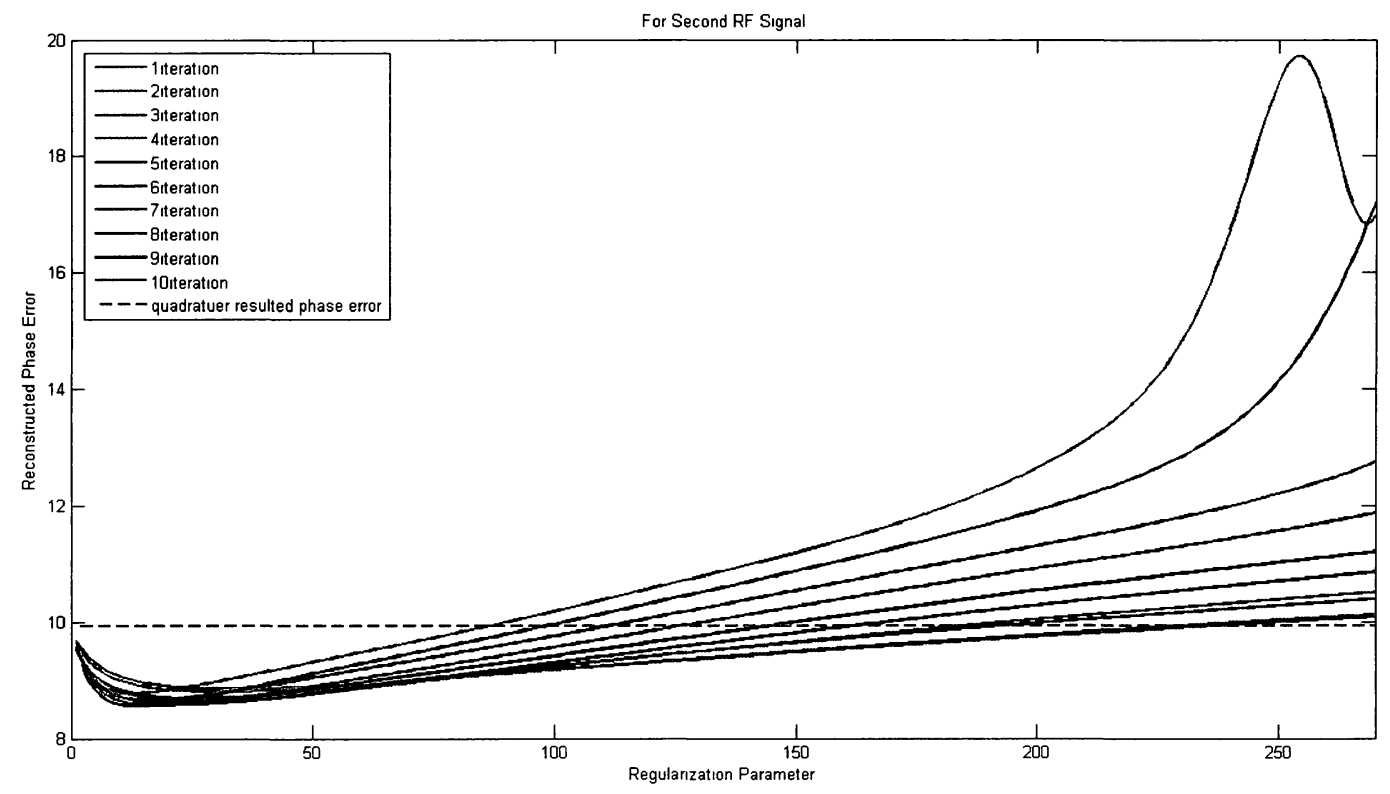

Figure 6.4 Reconstructed phase error $\left(\varepsilon_{2}\right)$ of the second (post-compression) RF signal (SNR $=10 \mathrm{~dB}$ ) with respect to different regularization parameters. Different colors demonstrate different number of iterations. Dashed line is the quadrature phase error $\left(\varepsilon_{1}\right)$ which determines the accepted regions for selecting the regularization parameters.

In the next two figures, the SNR level of the received RF signals is reduced to $3 \mathrm{~dB}$. This time, phase information of the first (pre-compression) RF signal and the phase shift occurred between the first two (pre- and post-compression) RF signals are represented in Figures 6.5 and 6.6, respectively. Again, the phase and phase shift plots are obtained by different methods of reference, quadrature and GN algorithm and shown in three different colors. 

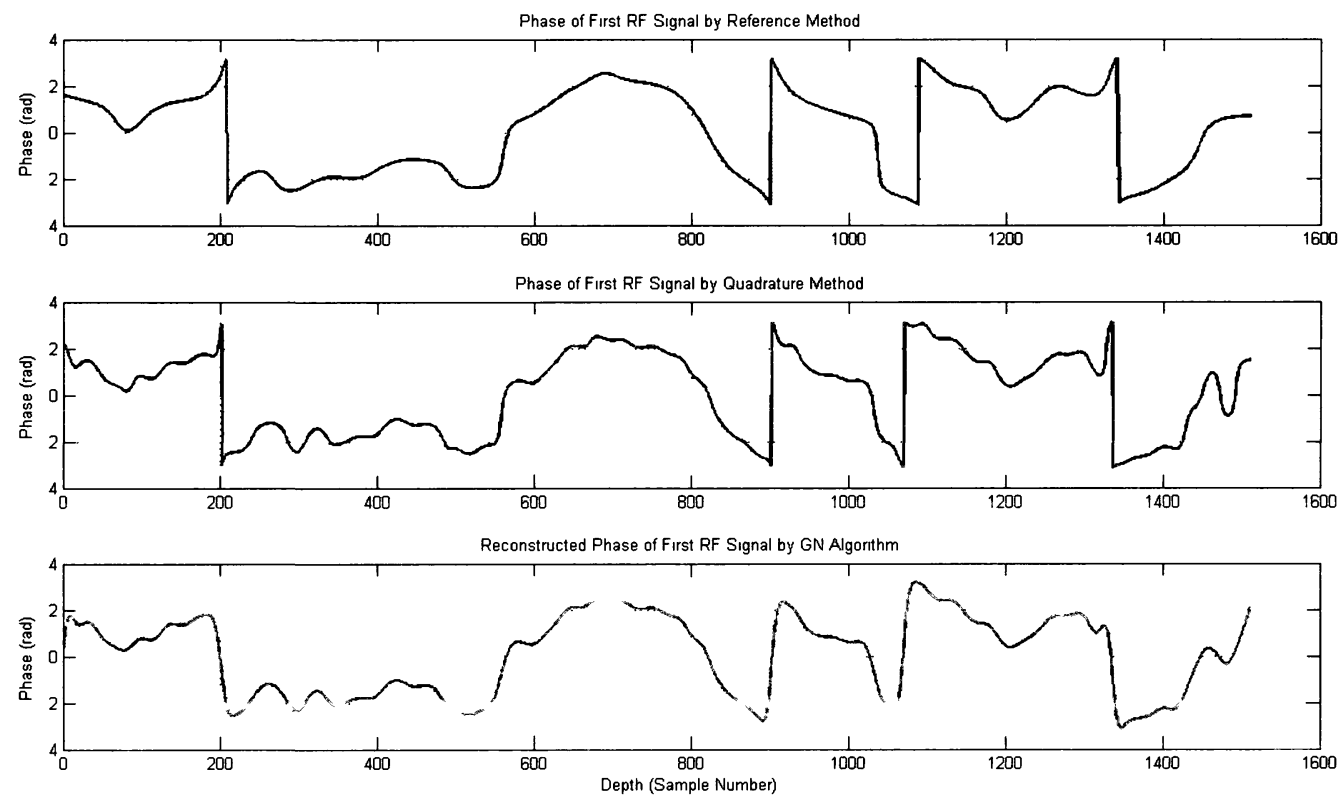

Figure 6.5 Phase of the fist (pre-compression) RF signal ( $S N R=3 \mathrm{~dB}$ ). Phase information resulted via the reference method (top), phase resulted from the quadrature method (middle), and the reconstructed phase obtained by GN algorithm (bottom).
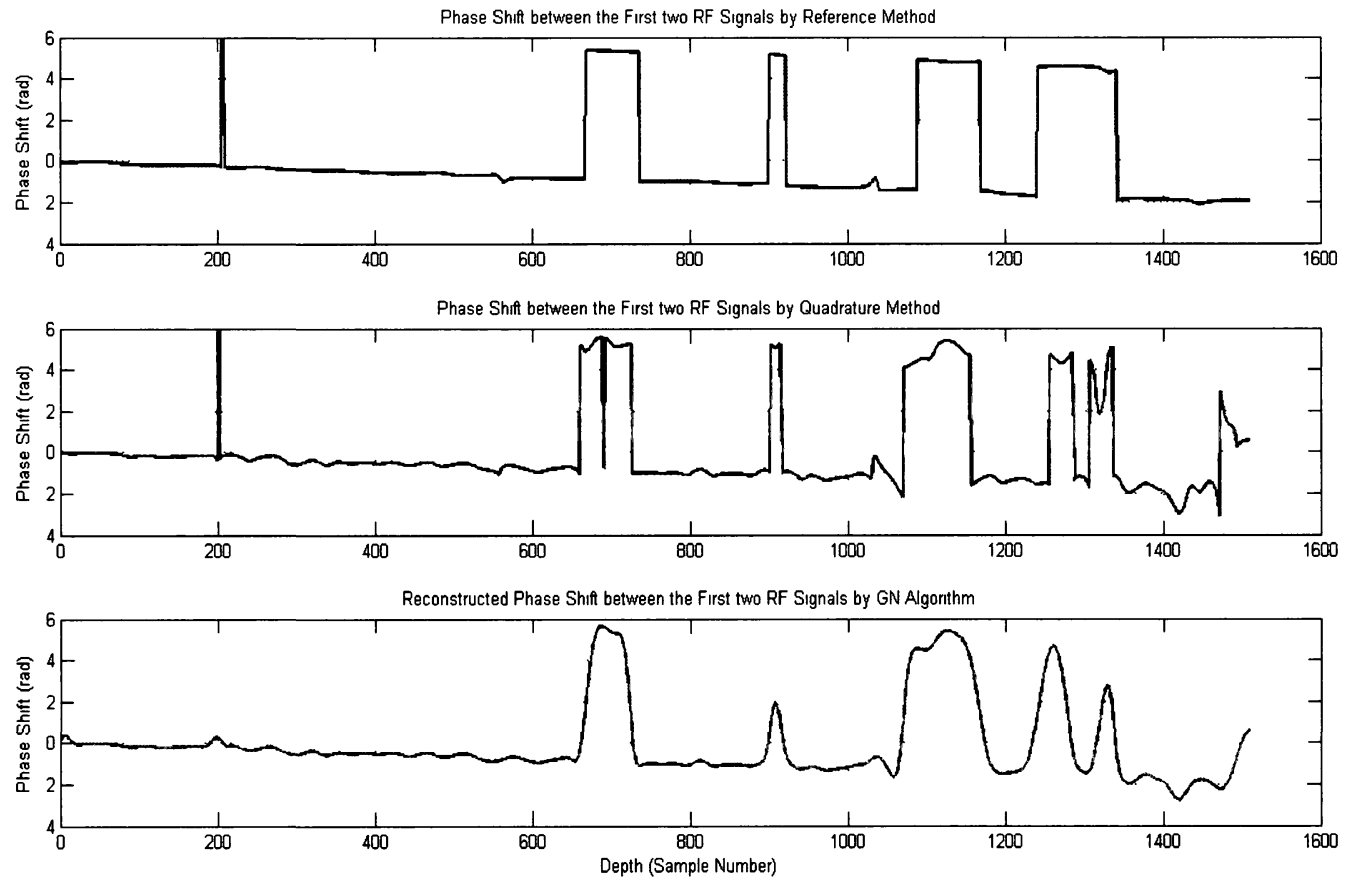

Figure 6.6 Phase shift between the first two (pre- and post-compression) RF signal ( $\mathrm{SNR}=3 \mathrm{~dB}$ ). Phase shift obtained by the reference method (top), phase shift resulted from the quadrature method (middle), the reconstructed phase shift obtained by the GN algorithm application (bottom). Regularization parameters of the GN algorithms applied on each of the two RF signals are optimal. 
For the first RF signal with the SNR of $3 \mathrm{~dB}$ (Figure 6.5), the optimal regularization parameter and the numeber of iterations were found to be 19 and 1, respectively. Again, these selections were made based on the reconstructed phase error $\left(\varepsilon_{2}\right)$ which was plotted with respect to different regularization parameters and iterations number (Figure 6.7).

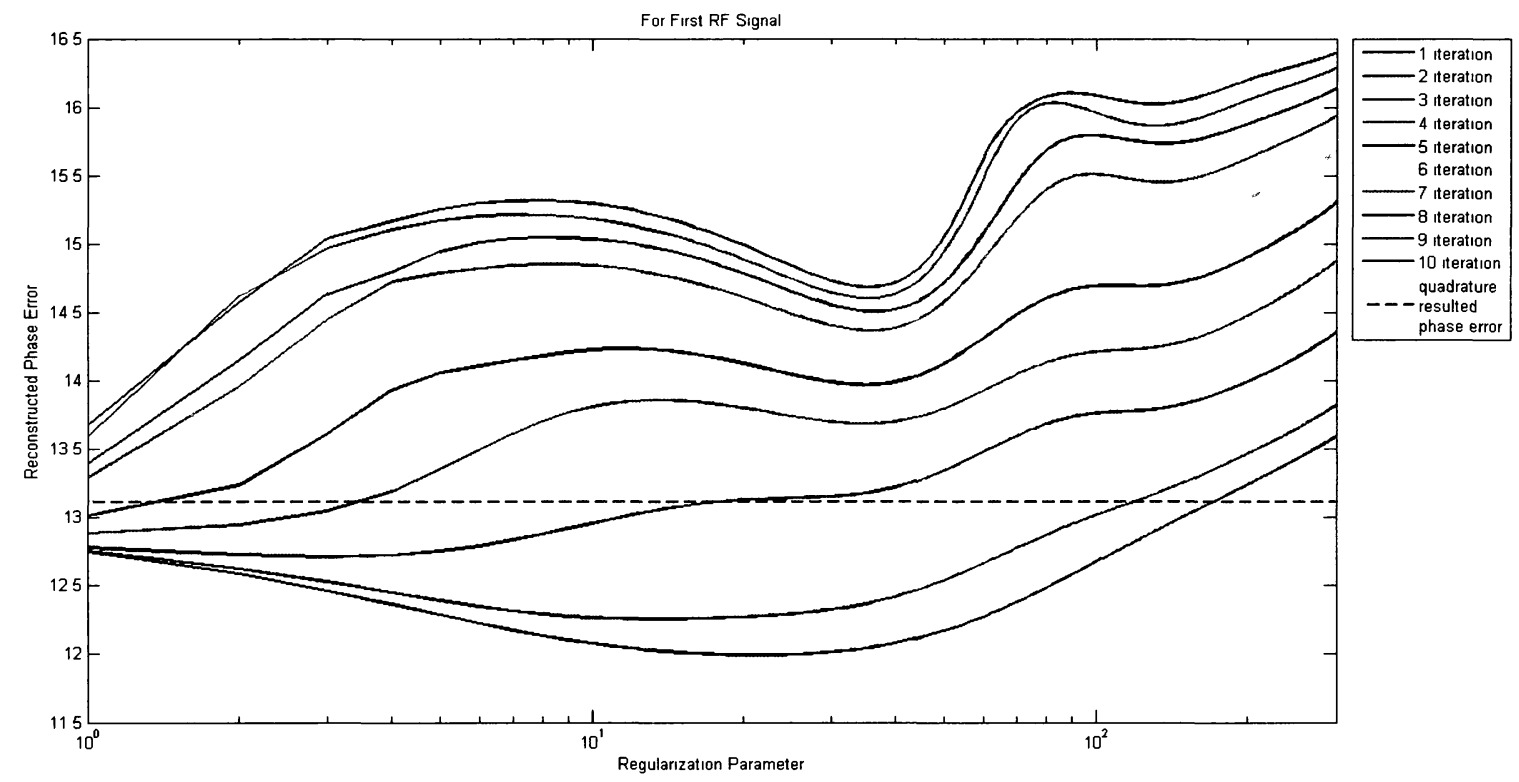

Figure 6.7 Reconstructed phase error $\left(\varepsilon_{2}\right)$ of the first RF signal $(\mathrm{SNR}=3 \mathrm{~dB})$ with respect to different regularization parameters. Different colors demonstrate different number of iterations. Dashed line is the quadrature phase error $\left(\varepsilon_{1}\right)$.

In tabels 6.1 and 6.2 , different regularization parameters, number of iterations, quadrature (observed) phase error $\left(\varepsilon_{1}\right)$, and reconstructed phase error $\left(\varepsilon_{2}\right)$ are represented for both the first and second RF signals with different SNR values. 
TABLE 6.1

\begin{tabular}{|c|c|c|c|c|}
\hline \multicolumn{5}{|c|}{$\varphi_{1}:$ Phase of the First RF signal } \\
\hline $\begin{array}{c}\text { SNR } \\
(\mathrm{dB})\end{array}$ & $\begin{array}{c}\text { Regularization } \\
\text { Parameter }\left(\mu_{1}\right)\end{array}$ & $\begin{array}{c}\text { Iteration } \\
\text { Number }\end{array}$ & $\begin{array}{c}\text { Quadrature Phase Error } \\
(\mathrm{rad}) \\
\varepsilon_{1}=\left\|\varphi_{1(\text { noisy })}-\varphi_{1(\text { rcal })}\right\|\end{array}$ & $\begin{array}{c}\text { Reconstructed Phase Error } \\
(\mathrm{rad})\end{array}$ \\
$\varepsilon_{2}=\left\|\mathbf{m}_{\text {estimated }}-\varphi_{1(\text { real })}\right\|$ \\
\hline 20 & 1 & 1 & 2.17 & 2.16 \\
\hline 10 & 15 & 1 & 7.64 & 6.81 \\
\hline 3 & 24 & 1 & 13.10 & 11.99 \\
\hline 0 & 28 & 1 & 15.17 & 14.03 \\
\hline
\end{tabular}

TABLE 6.2

$\varphi_{2}:$ Phase of the Second RF signal

\begin{tabular}{|c|c|c|c|c|}
\hline $\begin{array}{c}\text { SNR } \\
(\mathrm{dB})\end{array}$ & $\begin{array}{c}\text { Regularization } \\
\text { Parameter }\left(\mu_{2}\right)\end{array}$ & $\begin{array}{c}\text { Iteration } \\
\text { Number }\end{array}$ & $\begin{array}{c}\text { Quadrature Phase Error } \\
(\mathrm{rad})\end{array}$ & $\begin{array}{c}\text { Reconstructed Phase Error } \\
(\mathrm{rad})\end{array}$ \\
\hline 20 & 7 & 2 & 4.55 & 4.47 \\
\hline 10 & 14 & 9 & 9.93 & 8.56 \\
\hline 3 & 25 & 8 & 16.53 & 12 \\
\hline 0 & 30 & 6 & 20.21 & 12.11 \\
\hline
\end{tabular}


White Gaussian noise was added to the received RF signals such that the signal to noise ratio (SNR) varied from 0 to $40 \mathrm{~dB}$. Then, the quadrature phase shift error $\left(\Delta \varepsilon_{1}\right)$ and reconstructed phase shift error $\left(\Delta \varepsilon_{2}\right)$ were calculated (Figure 6.8). Note that in Figure 6.8 the norm of sub intervals were calculated and averaged to give the reconstructed or quadrature phase errors. The approach of error norm measurement (explained earlier in this chapter) is different from the one used in chapter 5 .

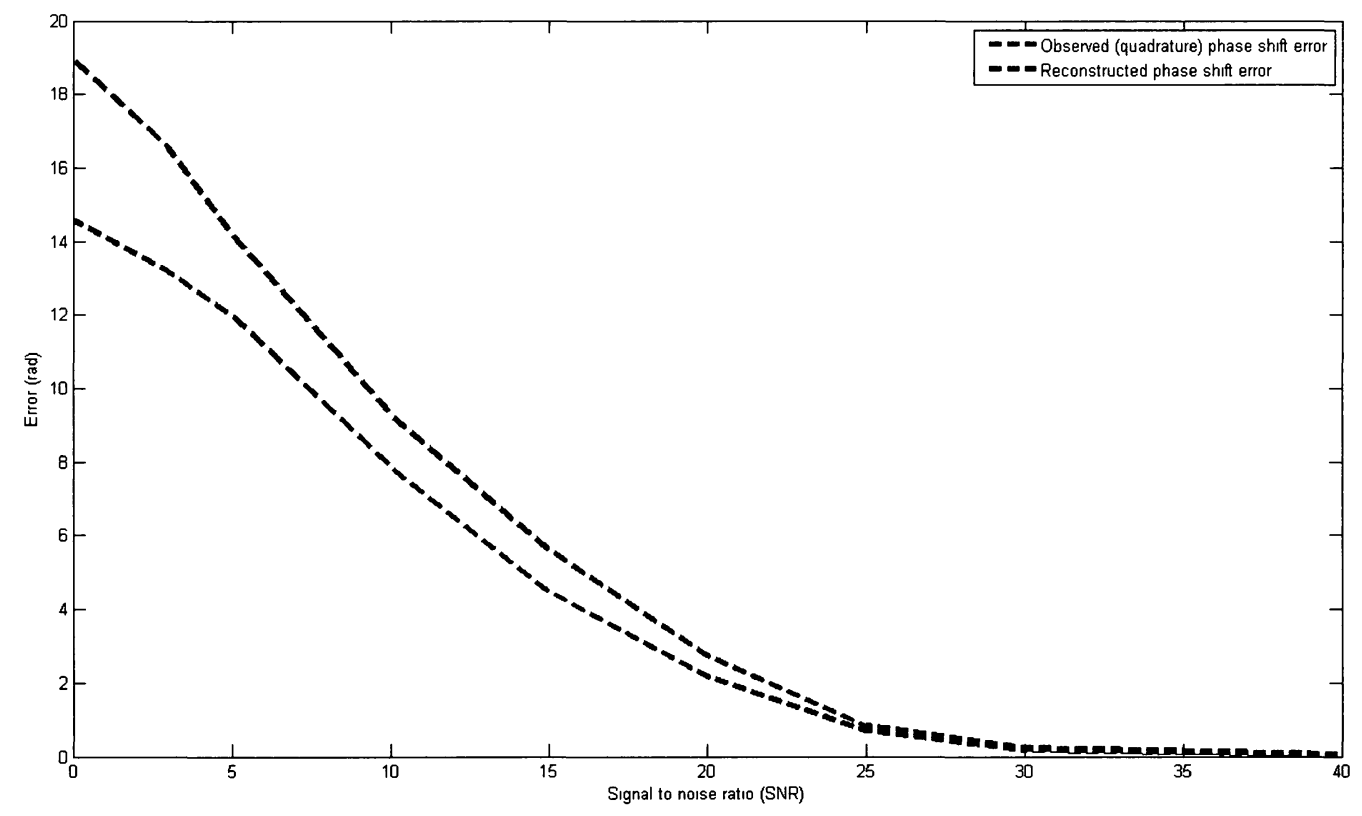

Figure 6.8 Reconstructed phase shift error (blue) and quadrature phase shift error (red) with respect to signal to noise ratio (SNR) of the RF signal.

It can be seen that defined GN algorithm can reconstruct the noisy phase results obtained via the quadrature technique making them closer to the real phase information. The effectiveness of the algorithm can be observed when the level of noise is increased. 


\subsubsection{Regularization Parameter Effect}

To investigate the effect of regularization parameter on the reconstruction process (algorithm), two extreme values of regularization parameters (very small and very large) were considered.

In Figure 6.9, the phase shift between the first two (pre- and post-compression) RF signals with the SNR of $3 \mathrm{~dB}$ is shown when large but still accepted regularization parameters were chosen for the GN algorithms. By accepted regularization parameter it means that the reconstructed phase shift error $\left(\Delta \varepsilon_{2}\right)$ is still less than the quadrature phase shift error $\left(\Delta \varepsilon_{1}\right)$ although the value of regularization parameter is increased, significantly.
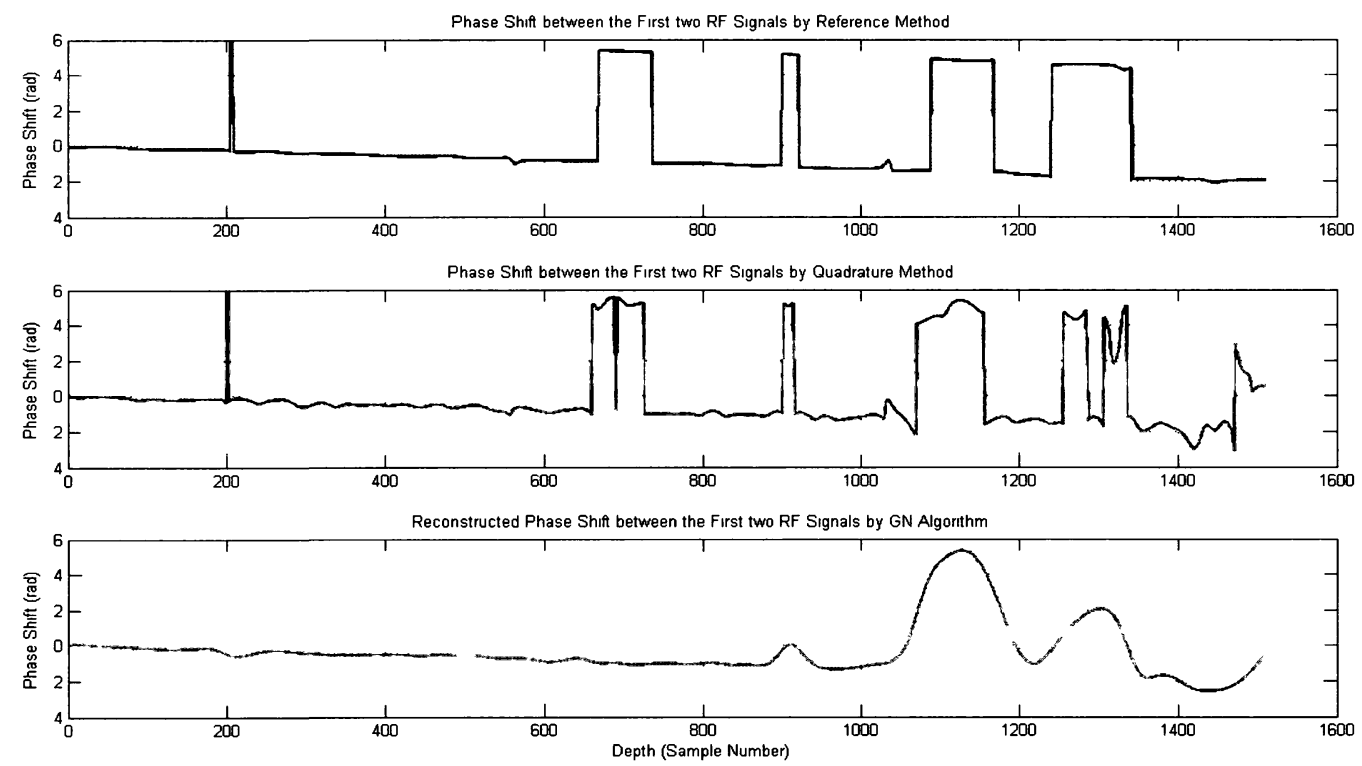

Figure 6.9 Phase shift between the first two (pre- and post-compression) RF signal (SNR = 3 dB). Phase shift obtained via the reference method (top), phase shift resulted from the quadrature method (middle), the reconstructed phase shift obtained by the GN algorithm (large regularization parameters $\left(\mu_{1}=166, \mu_{2}=165\right)$ ) (bottom). 

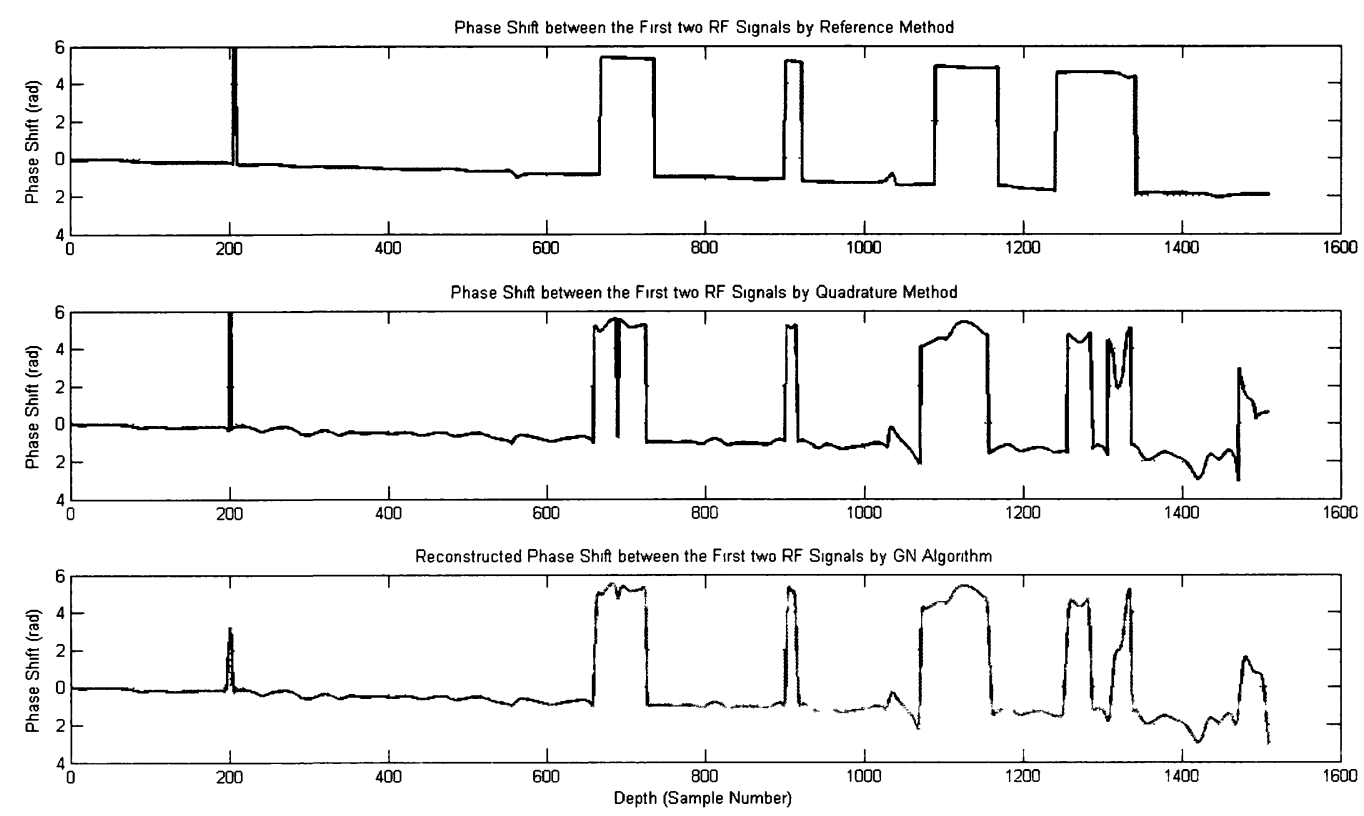

Figure 6.10 Phase shift between the first two (pre- and post-compression) RF signal (SNR $=3 \mathrm{~dB}$ ). Phase shift obtained via the reference method (top), phase shift resulted from the quadrature method (middle), the reconstructed phase shift obtained by the GN algorithm (small regularization parameters $\left(\mu_{1}=1, \mu_{2}=1\right)$ ) (bottom).

As it can be seen, small regularization parameter has almost no effect on the noisy phase (phase shift) plots obtained by the quadrature technique. By small regularization parameter, the GN algorithm cannot be considered as a phase reconstructive approach. However, when the regularization parameter is increased, the phase (phase shift) results become smoother, some of the $2 \pi$ jumps will be disappeared, and noise fluctuations will no longer be observed. For this reason, regularization parameter is often referred to the filter coefficient which can filter out the noise giving more accurate results.

It should be noted that extremely large regularization parameter will smooth the phase results enormously causing some phase information to be missed. In this way, the GN algorithm will no longer be effective due to the huge loss of information. 
Small and large regularization parameters used in the phase reconstruction algorithms along with the resulted errors $\left(\Delta \varepsilon_{1}\right.$ and $\left.\Delta \varepsilon_{2}\right)$ when different SNR levels are considered are represented in tables 6.3 and 6.4 .

TABLE 6.3

\begin{tabular}{|c|c|c|c|c|}
\hline \multicolumn{5}{|c|}{$\varphi_{1}:$ Phase of the First RF signal } \\
\hline $\begin{array}{l}\text { SNR } \\
\text { (dB) }\end{array}$ & $\begin{array}{l}\text { Regularization } \\
\text { Parameter }\left(\mu_{1}\right)\end{array}$ & $\begin{array}{l}\text { Iteration } \\
\text { Number }\end{array}$ & $\begin{array}{c}\text { Quadrature Phase Error } \\
\text { (rad) } \\
\varepsilon_{1}=\left\|\varphi_{1(\text { no } s y)}-\varphi_{1(\text { rcal })}\right\|\end{array}$ & $\begin{array}{l}\underset{(\mathrm{rad})}{\text { Reconstructed Phase Error }} \\
\varepsilon_{2}=\left\|\mathbf{m}_{\text {estımated }}-\varphi_{1(\text { real })}\right\|\end{array}$ \\
\hline 10 & 78 & 2 & 7.64 & 7.63 \\
\hline 10 & 1 & 9 & 7.64 & 7.37 \\
\hline 3 & 166 & 8 & 13.10 & 13.09 \\
\hline 3 & 1 & 6 & 13.10 & 12.75 \\
\hline
\end{tabular}


TABLE 6.4

\begin{tabular}{|c|c|c|c|c|}
\hline \multicolumn{5}{|c|}{$\varphi_{2}:$ Phase of the Second RF signal } \\
\hline $\begin{array}{c}\text { SNR } \\
(\mathrm{dB})\end{array}$ & $\begin{array}{c}\text { Regularization } \\
\text { Parameter }\left(\mu_{2}\right)\end{array}$ & $\begin{array}{c}\text { Iteration } \\
\text { Number }\end{array}$ & $\begin{array}{c}\text { Quadrature Phase Error } \\
(\mathrm{rad}) \\
\varepsilon_{1}=\left\|\varphi_{2(\text { noosy })}-\varphi_{2(\text { rcal })}\right\|\end{array}$ & $\begin{array}{c}\text { Reconstructed Phase Error } \\
(\mathrm{rad})\end{array}$ \\
$\varepsilon_{2}=\left\|\mathrm{m}_{\text {estumated }}-\varphi_{2(\text { real })}\right\|$ \\
\hline 10 & 95 & 2 & 9.93 & 9.87 \\
\hline 10 & 1 & 9 & 9.93 & 9.56 \\
\hline 3 & 165 & 8 & 16.53 & 16.47 \\
\hline
\end{tabular}

\subsection{Phase Jump Fixing}

In chapter 5 it was explained that certain phase jumps ( $2 \pi$ and less than $2 \pi)$ might be created in the phase or phase shift plots obtained by the quadrature method. It was explained that the creation of these jumps stems from a limitation that is existed in all phase measurement methods. Unwrapping is able to fix $2 \pi$ phase jumps while it fails when applies on the less than $2 \pi$ jumps. In Figure 6.11, phase shift between the $5^{\text {th }}$ simulated RF signal pairs is measured by the reference and quadrate method (top and bottom plots). In this figure, around the sample number of 558 a less than $2 \pi$ phase jump is created. To see if the GN algorithm can fix these types of jumps or not, a GN algorithm with the same description defined in this chapter but different regularization 
and iteration numbers was applied. The reconstructed phase shift plot by GN algorithm is shown at the bottom plot of the Figure 6.11 .
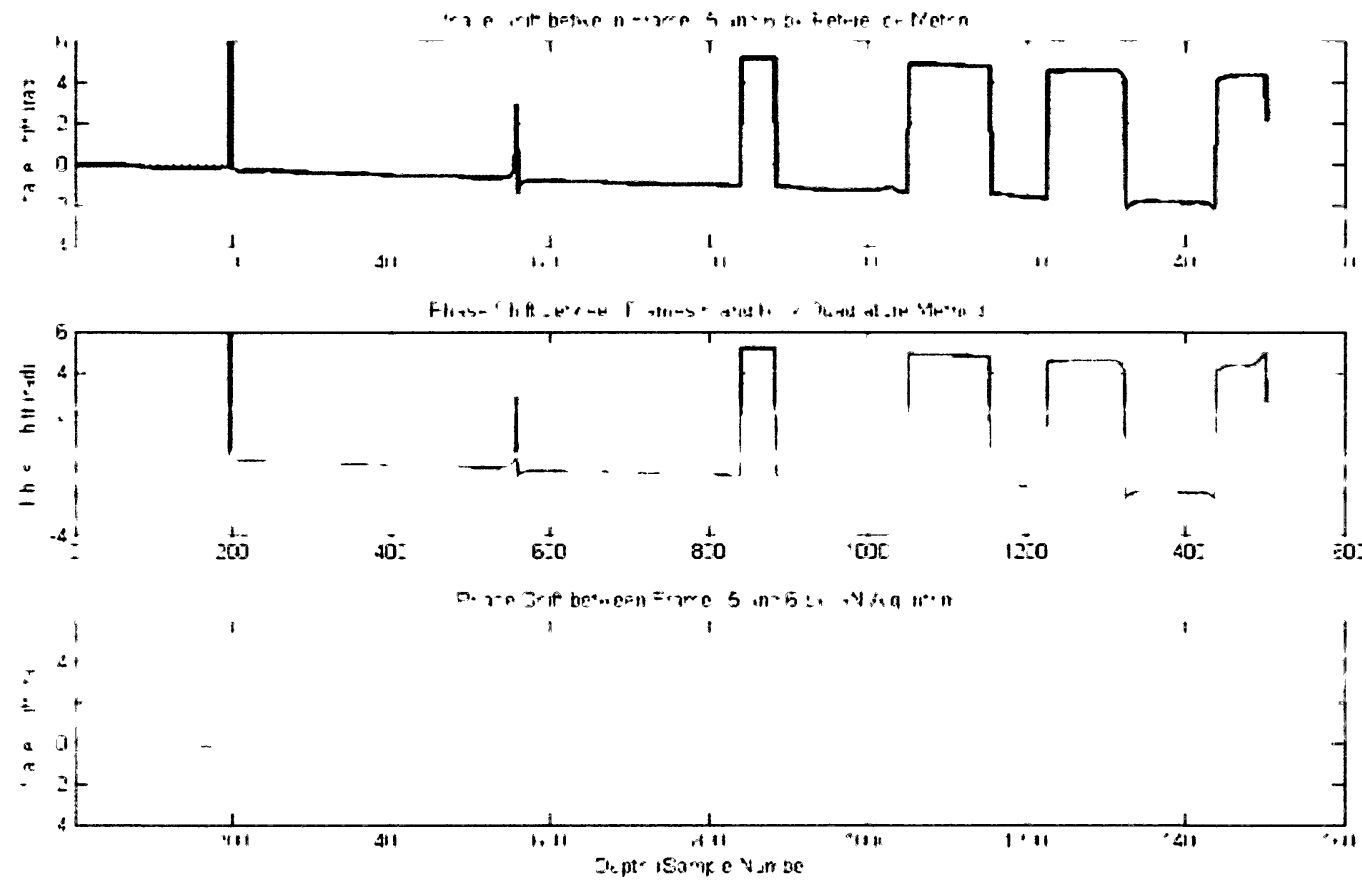

Figure 6.11 Phase shift between the $5^{\text {th }}$ RF signal pairs. Phase shift obtained via the reference method (top), phase shift resulted from the quadrature method (middle), the reconstructed phase shift obtained by the GN algorithm (bottom). In this figure the $\pi$ phase jump at sample number 558 is fixed by the GN algorithm

It can be seen that the GN algorithm not only is able to remove the noise and act like a filter, but also it can fix phase jumps, especially those that cannot be fixed by unwrapping (less than $2 \pi$ jumps).

\subsection{Comparison with the Linear Lowpass Filter}

Instead of nonlinear GN algorithm, a linear lowpass filter could be applied on the phase shift results to remove the effect of noise. However, due to nonlinear nature of the phase, the GN algorithm was expected to function better. To compare the functionality of linear filter (LPF) 
with nonlinear filter (GN algorithm), a lowpass Butterworth filter was defined and applied on the noisy phase shift results obtained by the quadrature method. Throughout the examination, the cutoff frequency of the lowpass filter was varied. With cutoff frequencies less than $0.3 \mathrm{MHz}$ and greater than $1 \mathrm{MHz}$, huge loss of phase information and noisy phase outcomes were observed, respectively. Between these values, the cutoff frequency of $0.7 \mathrm{MHz}$ was resulted in the phase shift outcome much closer to that of the GN algorithm, compare to other cutoff frequency values (Figure 6.12).

By comparing the results of filters, it was observed that the lowpass filter causes the $2 \pi$ phase jumps to become broader while their amplitudes are reduced, whereas the GN algorithm reduces the amplitude of the spikes without making them wider. Moreover, as the cutoff frequency of the lowpass filter decreases and in turn, the phase shift plots become smoother, increasing trend of the phase shift plots cannot be observed. Instead, a smooth line with rises and falls will be observed. This causes the lowpass filtered phase shift result to deviate from that of the reference method even though the phase shift plot is smoothened by lowpass filtering. 
Cutof Frequency $=87 \mathrm{MHz}$

Phase Shit between the Filst Iwo RF Signais by the Reference method

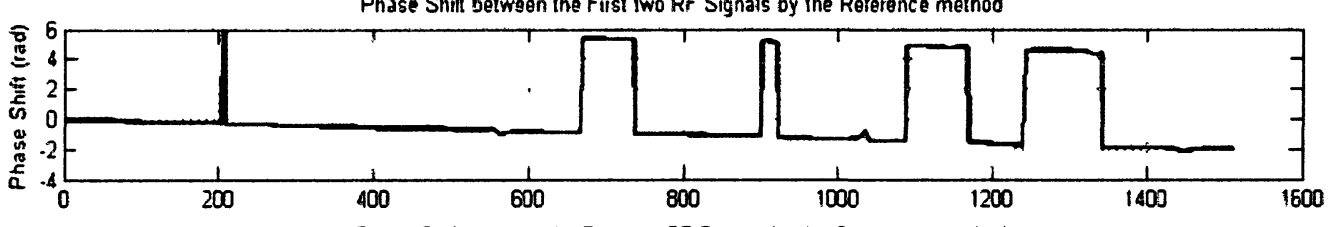

Phase Shit between the Firsl two RF Signals by the Quadrature method

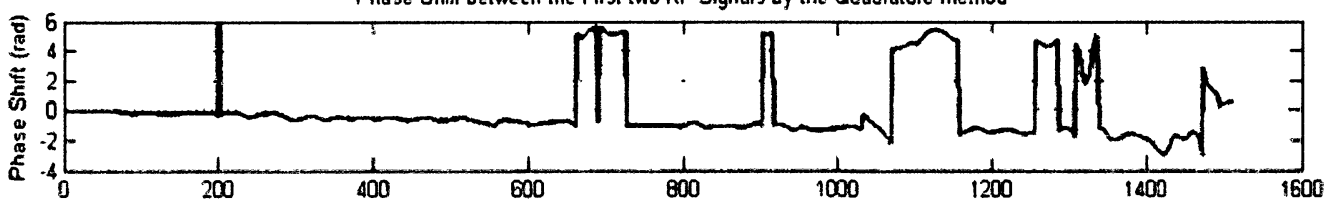

Phase Shin vetweer the F is! two RF Signals aftel Applying the LPF

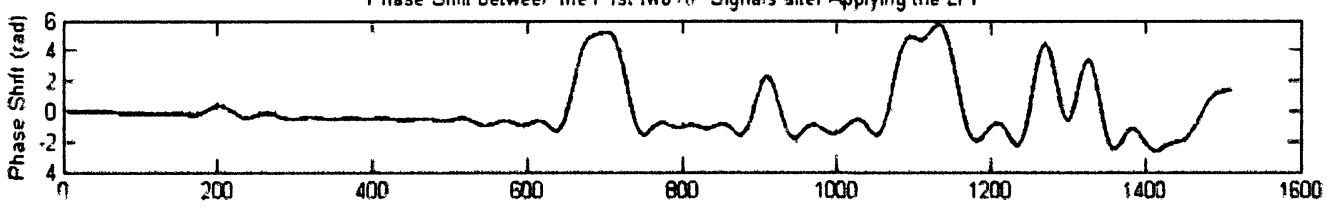

Phase Shet between the First iwo RF Signals after applying the GN Algonthm

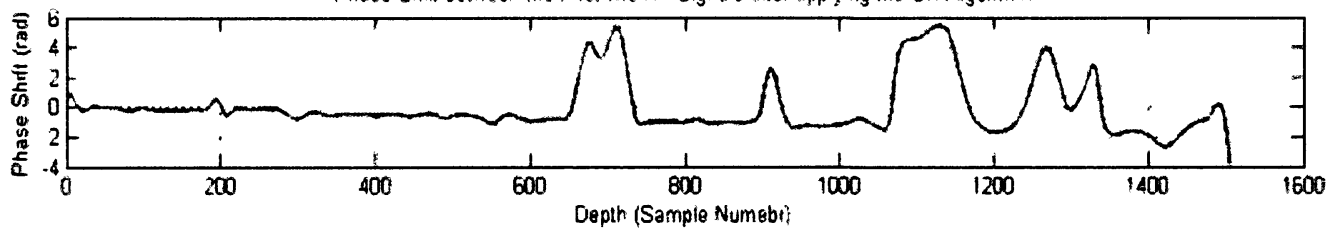

Figure 6.12 Phase shift between the first two RF signals. Phase shift obtained via the reference method (blue), phase shift resulted from the quadrature method (red), the lowpass filtered phase shift (green), and the reconstructed phase shift by the GN algorithm (purple). The lowpass filter has the cutoff frequency of $0.7 \mathrm{MHz}$. the regularization parameter of GN algorithm is optimal. 


\section{Chapter 7}

\section{Phantoms and Results}

The eventual goal of this research involves application in ultrasound real data measurements. Although the majority of work was done by computer simulation to develop and verify the GN algorithm and its functionality, some preliminary work was done on the real data measurements. This step demonstrates the effectiveness of the defined GN algorithm on the data obtained from compressed (expanded) ultrasound experimental phantoms that simulate muscle contraction state. The following chapter outlines the generalization of $\mathrm{GN}$ algorithm to the data measurements and illustrates samples of reconstructed phase corresponding to the measured RF signals.

\subsection{Measured RF Signals}

In this research, some of the measured data stored in ".zrf" files were used for the purpose of experimental testing. As mentioned in chapter 3, these data (RF-signals) were obtained in previous works where an ultrasound probe was used to compress (or expand) particular ultrasound phantoms simulating the skeletal muscles. By pushing down the probe on the phantom, muscle contraction was simulated.

During phantom compression (expansion), when the ultrasound system was in M-mode operation, $5550 \mathrm{RF}$-signals or equivalently, $5549 \mathrm{RF}$ signal pairs were created and stored. RF signals included in each pair are called as pre- and post-compression (expansion) RF signals. Compressing the phantom causes the post-compression RF signals to become shorter (in time or 
depth) than the pre-compression RF signals. In contrast, phantom expansion results in a longer post-expansion $\mathrm{RF}$ signals relative to the pre-expansion ones.

\subsection{Phase Reconstruction Process}

Figures 7.1 and 7.2 represent the first two measured RF signals (corresponding to the first and second scan lines) along with their phase information.
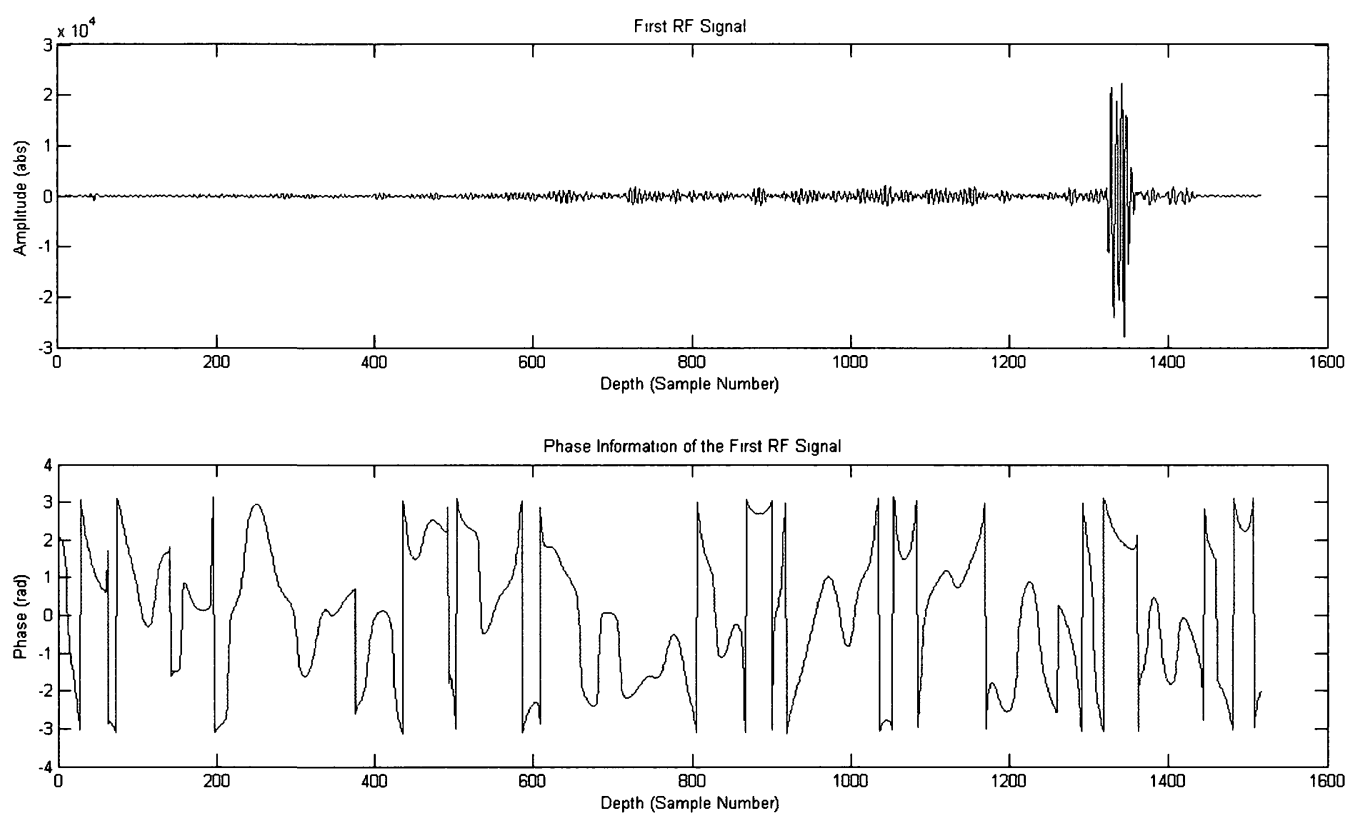

Figure 7.1 First (pre-compression) measured RF signal (top), corresponding to the first scan line (or frame). Phase information of the first measured RF signal (bottom) by the quadrature method. 

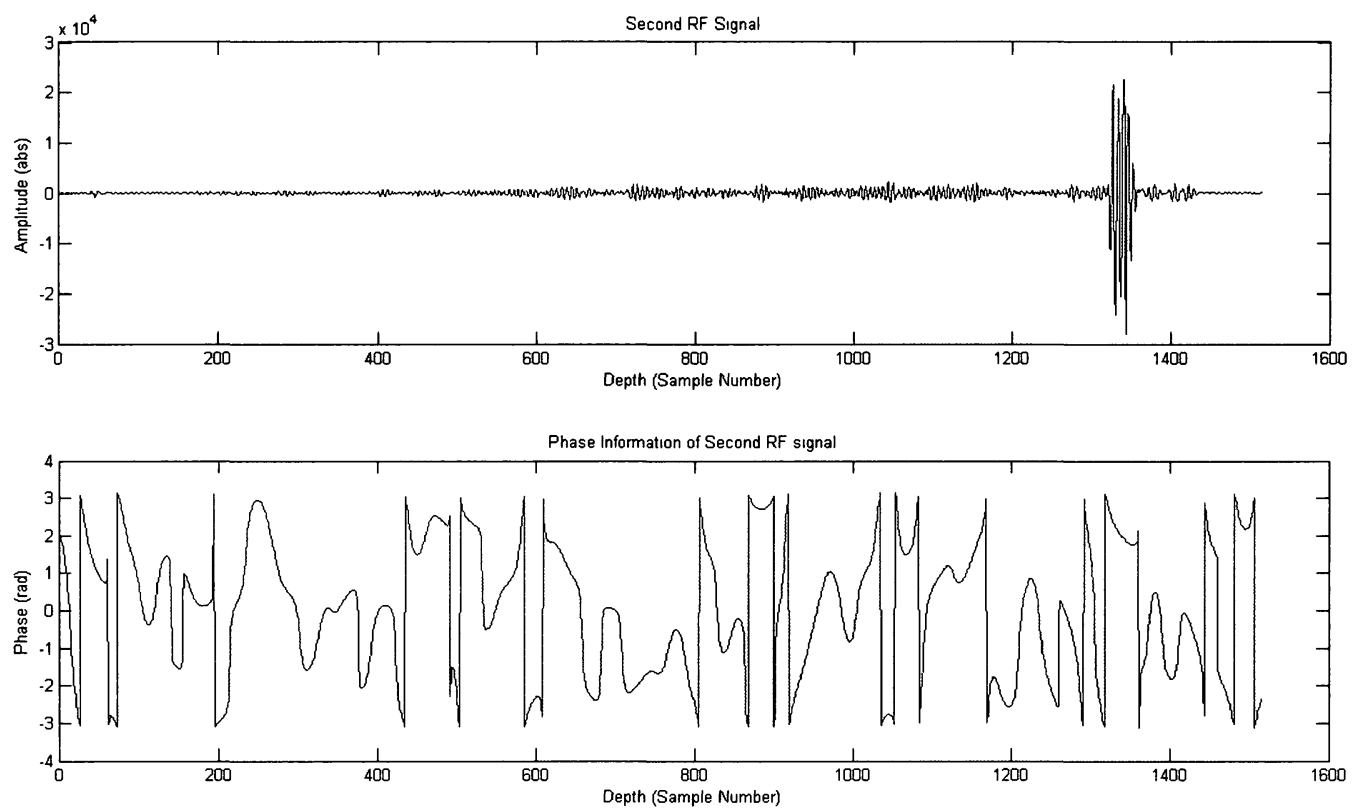

Figure 7.2 Second (post-compression) measured RF signal (top), corresponding to the second scan line (or frame). Phase information of the second measured RF signal (bottom) by the quadrature method.

As it can be seen from the results, the phase information of the first two RF signals (pre- and post-compression RF signals) are quite similar. It demonstrates that during compression/expansion of the sample phantom, the probe moved very slowly. Therefore, the amount of displacement of the post-compression RF signal will be very small relative to the precompression RF signal. Similarly, the consecutive phase shift between these two signals is expected to be very small (Figure 7.3). 


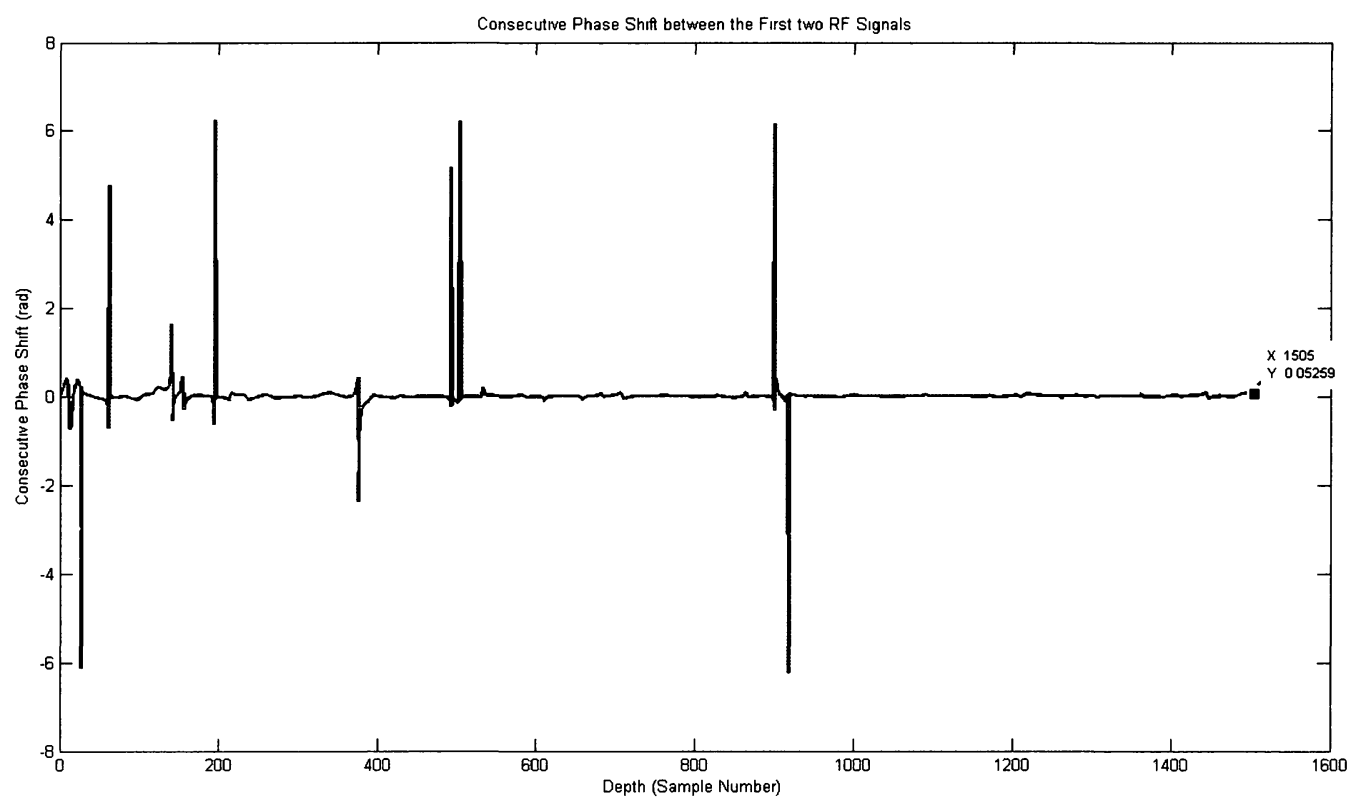

Figure 7.3 Consecutive phase shift between pre- and post-compression RF signals, measured by the quadrature phase detection technique.

Again, since the consecutive phase shift shown in Figure 7.3 does not change significantly at different depth sample numbers, it becomes apparent that the phantom was expanded gradually during RF signal generation. Consecutive phase shift at the maximum depth sample number is a small positive value which demonstrates that the experimental phantom was expanded.

Since the probe was gradually applied on the phantom, it is expected that the phase information of other RF signals to be very similar (with small changes) to their prior pair. Therefore, for reconstructing the phase information of the measured data, one single GN algorithm can be defined and applied to all generated 5550 RF signals. In Figures 7.4 and 7.5, reconstructed consecutive phase shift between the first two generated RF signals is shown when regularization parameter in the GN algorithm is equal to 1 and 50, respectively. 


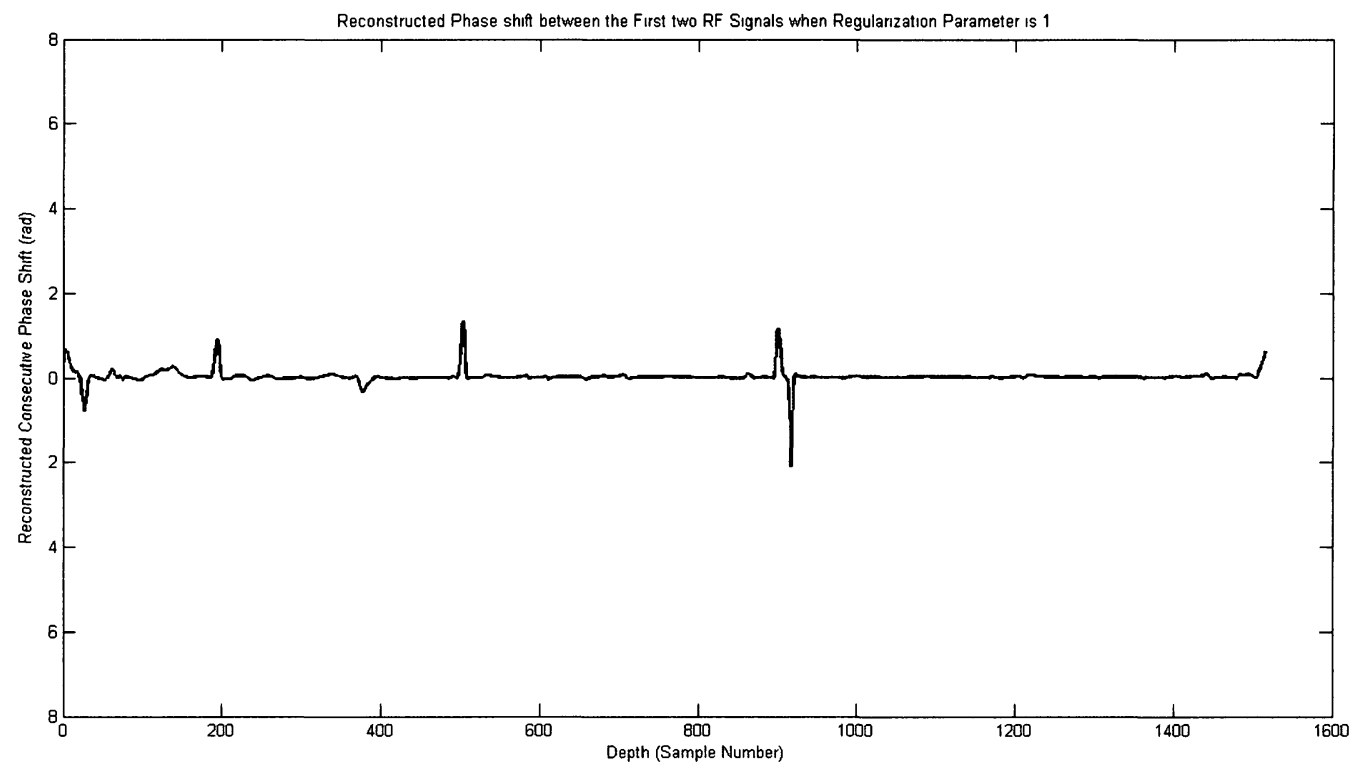

Figure 7.4 Reconstructed consecutive phase shift between the first two (pre- and post-compression) RF signals (frames), resulted from applying the GN algorithm with regularization parameter of 1.

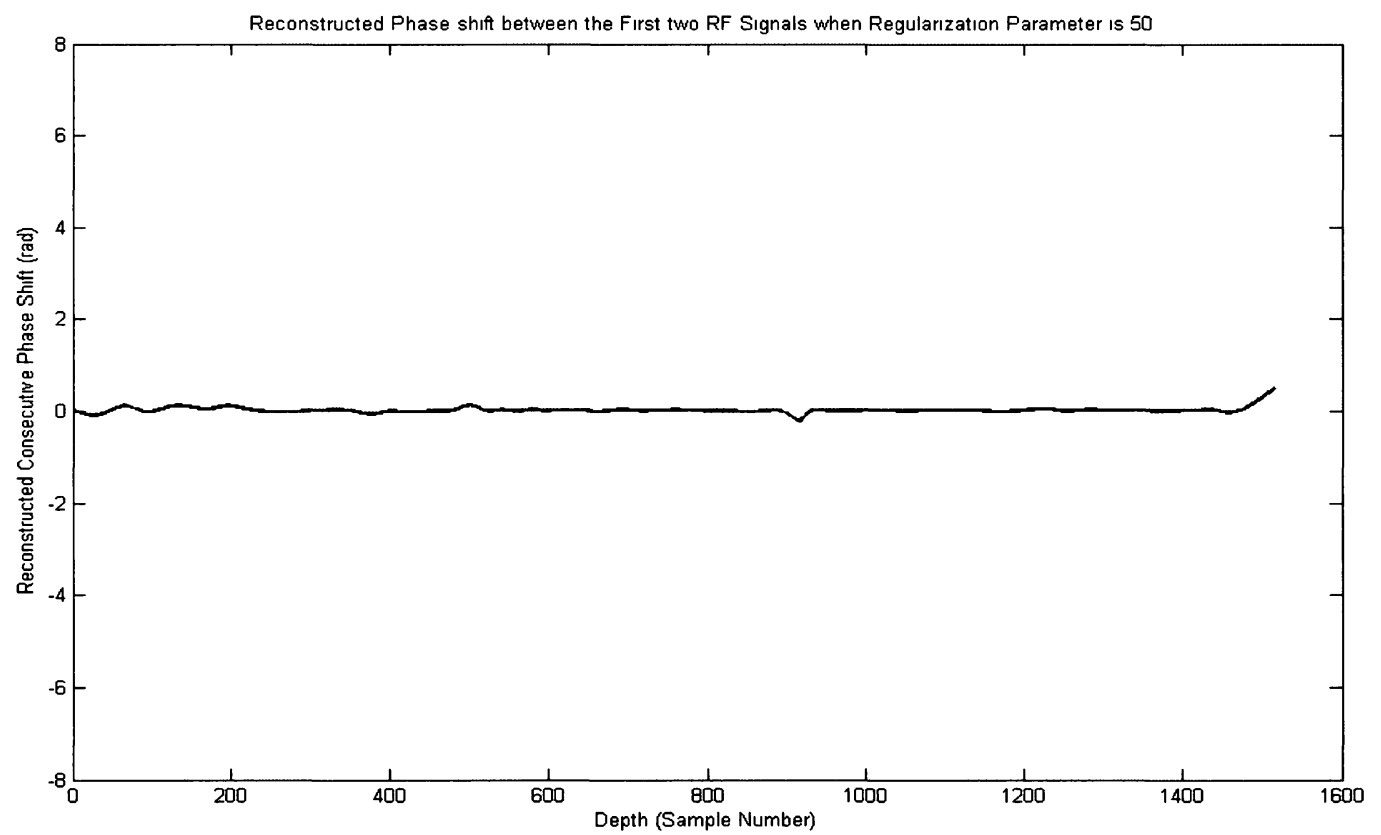

Figure 7.5 Reconstructed consecutive phase shift between the first two (pre- and post-compression) RF signals (frames), resulted from applying the GN algorithm with regularization parameter of 50. 
By comparing Figures 7.3 and 7.4 , it becomes evident that even a small regularization parameter, such a 1, can affect the measured consecutive phase shift fixing its phase jumps, significantly. Then, the regularization parameter increased to a greater value (50) (Figure 7.5). As explained in chapter 6, regularization parameter in defined GN algorithm can be considered as a filter coefficient. Large value regularization parameters will therefore, have greater influence on the noisy phase information which are aimed to be reconstructed. It can be observed that the GN algorithm with large regularization parameter removes almost all phase jumps observed in Figure 7.3.

Similarly, defined GN algorithm was applied to all 5550 measured RF signals. In the following figures, the accumulated phase shift after 5550 frames is represented before and after applying the GN algorithm.

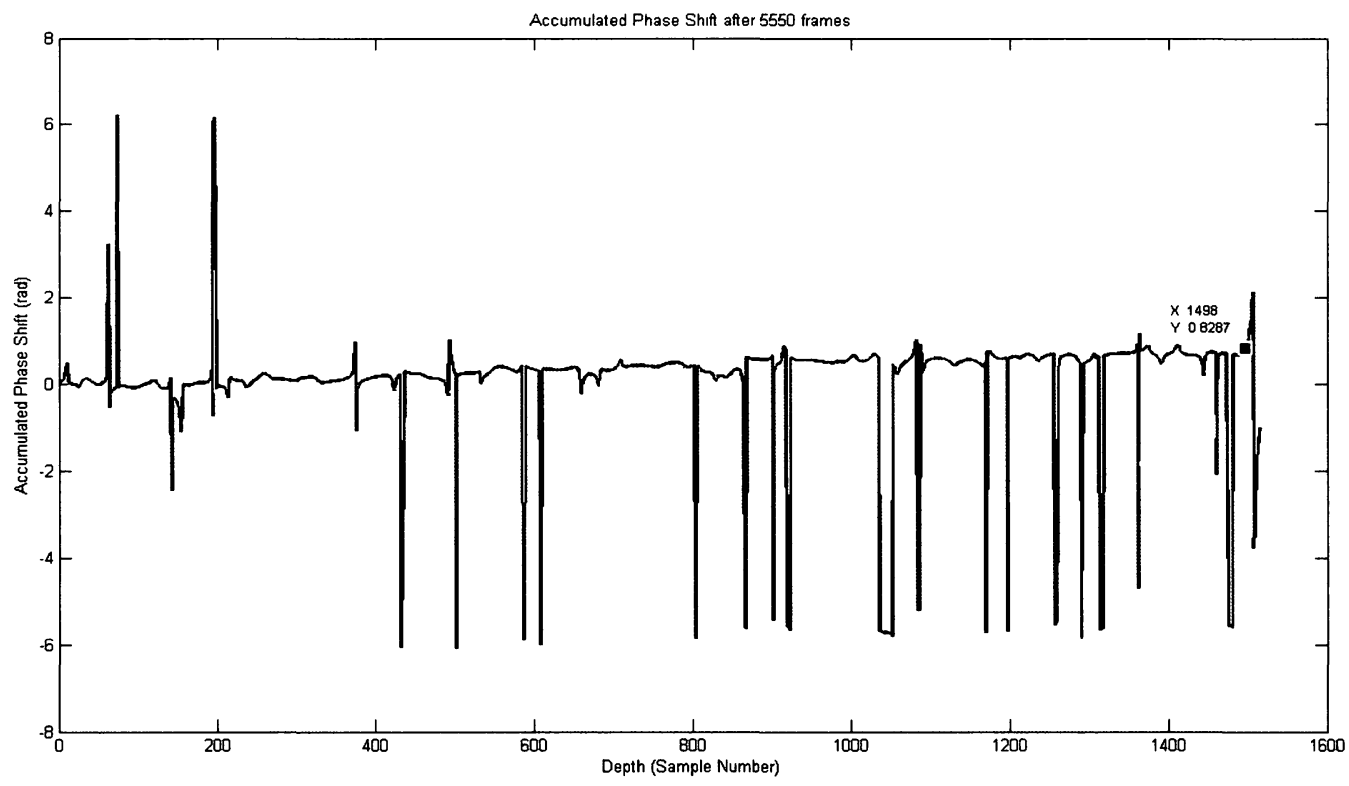

Figure 7.6 Accumulated phase shift after 5550 frames, measured by the quadrature detection method. 
The accumulated phase shift plot is the result of adding all 5549 consecutive phase shift plots corresponding to 5549 RF signal pairs. Even though the slope in each of these consecutive phase shift plots was not considerable, it becomes noticeable if these are all added up. Therefore, the slope of accumulative phase shift shown in Figure 7.6 will become noticeable.

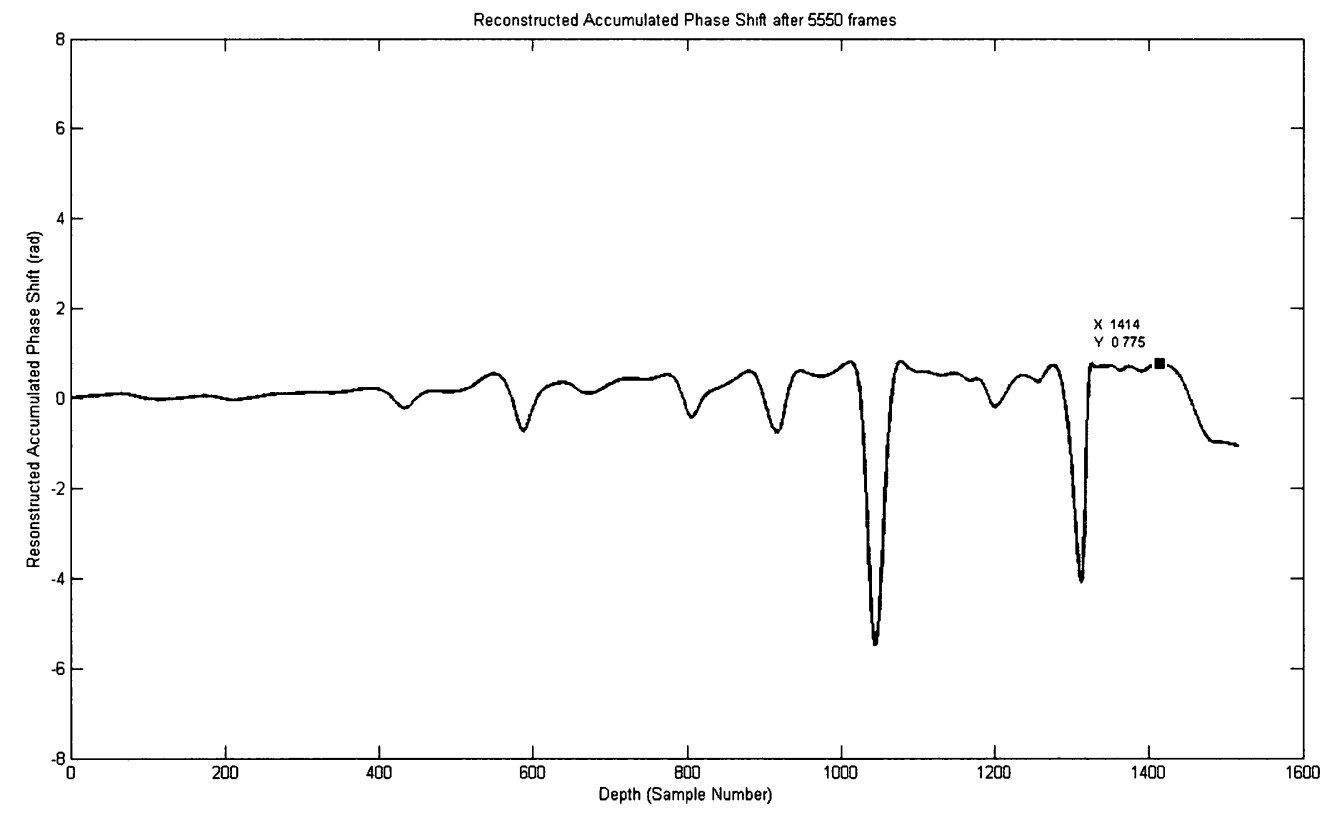

Figure 7.7 Accumulated phase shift after 5550 frames, resulted from the GN algorithm with regularization parameter of 100 . 


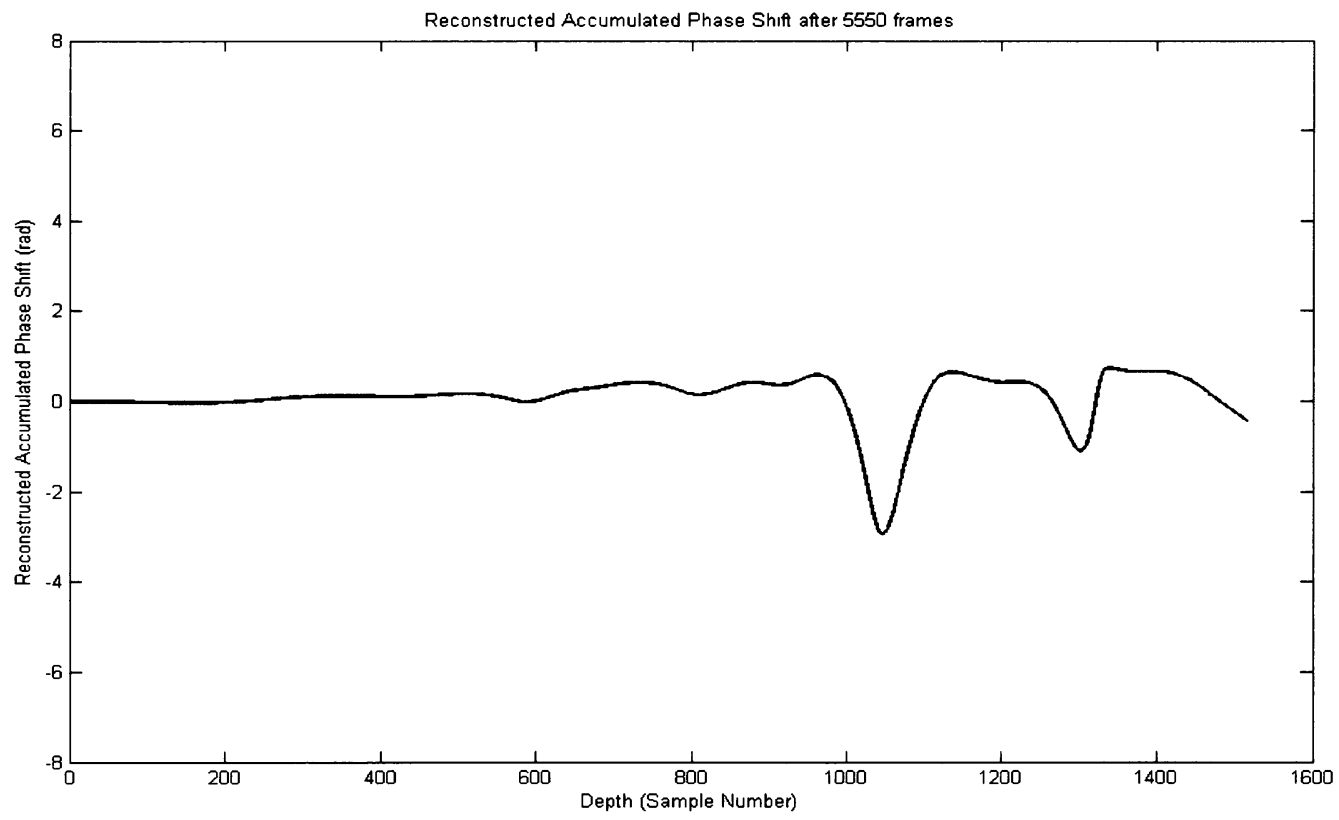

Figure 7.8 Accumulated phase shift after 5550 frames, resulted from applying the GN algorithm with high regularization parameter equal to 1000 .

An appropriately defined GN algorithm is able to remove noise and fix phase jumps, considerably. However, it should be noted that although the incorporated regularization parameter can filter out the noise, it can also remove some phase information. Therefore, there has to be a tradeoff between high and low value regularization parameter if we want to have an optimal reconstructive GN algorithm. 


\section{Chapter 8}

\section{Conclusions and Future Works}

\subsection{Conclusion}

In this research, it was tried to measure the phase information of ultrasonic received RF signals accurately. Quadrature phase detection technique, which is a method commonly used to measure the phase information, was tested in different conditions. Parameters that affect its functionality causing its outcomes to become less accurate were explored. Among the explored affecting parameters, signal to noise ratio of the received RF signal was considered for further examination. It was observed that by increasing the level of noise, the phase results obtained by the quadrature technique would become less accurate and deviate from the real phase which is aimed to be obtained. To resolve this problem, a novel phase reconstructive approach was defined based on the Gauss-Newton algorithm. In this algorithm, certain parameter, known as regularization parameter, were varied to study its effect on the process of reconstruction. It becomes evident that the regularization parameter can act as a filter coefficient able to remove a part of noise appeared in the phase results. Extremely low value of it had no impact on the phase information. i.e., didn't change noisy phase representation, considerably. E \xtremely large value of it however, can result in the loss of phase information. Indeed, it can remove a portion of phase information while tries to cut out the noise. Therefore, it is always desired to find an approach by which the optimal value of regularization parameter can be selected. There are several approaches that can search for the optimal regularization parameter. However, not all of 
them can work for all problems. For instance, in this research it was not possible to apply Lcurve criterion (one of the different approaches of finding optimal regularization parameter) since the conditions of this criterion were not provided in this work.

\subsection{Future Works}

- Studying on different approaches of regularization parameter selection and finding the one that can be applied on ultrasound phase reconstruction process is remained as a future work.

- In this research, just a few number of iterations were examined due to the computer speed problem. Improving the designed algorithm in a way that greater number of iterations can be studied is another work that can be done in future.

- Examination of other parameters that affect the quadrature method, such as the bandwidth of the signal is another step that is aimed to be taken in the future.

- This research simulates speckle echoes generated as the ultrasound beam is transmitted through a single layer of a part of body. In future, this simulation can be extended to cover different tissue layers of the body, such as muscle and bone layers. 


\section{Reference}

Aster, R. C., Thurber, C. H., \& Borchers, B. (2005). Parameter estimation and inverse problems. United Kingdom: Elsevier Academic Press.

Aarås, A. (1994). The impact of ergonomic intervention on individual health and corporate prosperity in a telecommunications environment. Ergonomics, 37(10), 1679-1696.

Benjamin, P. J., \& Lamp, S. P. (2005). Understanding sports massage. (2nd ed.). United States: Human Kinetics.

Bhargava, S. K. (2002). Principles and practice of ultrasonography. New Delhi (India): Jaypee Brothers Medical Publishers.

Bae, U., \& Kim, Y. (2007). Angular strain estimation method for elastography. IEEE Transaction on Ultrasonics, Ferroelectrics and Frequency Control. 54(12), 2653-2661.

Chang, S. H., Park, S. B., \& Cho, G. H. (1993). Phase-error-free quadrature sampling technique in the ultrasonic B-scan imaging system and its application to the synthetic focusing system. IEEE Trans. Ultrason.,Ferroelect., Freq. Contr., 40( 3), 216-223.

Contreras, S. H., Macione, J., Fox, M. D. (2009). Displacement estimation in ultrasound images using pseudo-phase. Proceedings of the 35th Annual Northeast Conference of the IEEE EMBS.

Catheline, S., Wu, F., \& Fink, M. (1999). A solution to diffraction biases in sonoelasticity: the acoustic impulse technique. Acoust. Soc. Am., 105(5), 2941-50.

Dansereau, R. M. (2009). Introduction to digital signal processing. (8th ed.). Canada: Carleton University. 
D'hooge, J., Heimdal, A., Jamal, F., Kukulski, T., Konofagou, B., Bijnens, F., et al. (2000). Regional Strain and Strain Rate Measurements by Cardiac Ultrasound: Principles, Implementation and Limitations. Eur J Echocardiogr, 1(3), 154-170.

De Ana, F. J., \& O'Donnel, M. (2005). Quantitative real-time blood flow estimation with intravascular ultrasound in the presence on in-plane flow. IEEE Transaction on Ultrasonics, Ferroelectrics and Frequency Control. 52(11), 1952-1961.

Demirli, R. \& Saniie, J. (2001). Model-based estimation of ultrasonicechoes part I: analysis and algorithms. IEEE Trans. Ultrason.,Ferroelect., Freq. Contr., 48, 787-802.

Demirli, R., \& Saniie, J. (2001). Model-based estimation of ultrasonic echoes part II: nondestructive evaluation applications. IEEE Trans. Ultrason.,Ferroelect., Freq. Contr., 48(3), 803-811.

Engl, H. W., Hanke, M., \& Neubauer, A. (2005). Regularization of inverse problems. Philadelphia: SIAM.

Engl, H. W., \& Kügler P. (2005). Nonlinear inverse problems: theoretical aspects and some industrial applications. Mathematics in Industry, 6(1), 3-47.

Farron, J., Varghese, T., \& Thelen, D. G. (2009). Measurement of tendon strain during muscle twitch contractions using ultrasound elastography, IEEE Trans. Ultrason.,Ferroelect., Freq. Contr., 56(1), 27-35.

Fry, H. J. H., (1986). Overuse syndrome in musicians; prevention and management. The Lancet, 328(8509), 728-731.

Feng, N., Zhang, J., \& Wang, W. (2006). A quadrature demodulation method based on tracking the ultrasound echo frequency. Ultrasonics, 44(1), e47-e50. 
Garrett, W. (1996). Muscle strain injuries. Am J Sports Med., 24(6), S2-8.

Guirong, G., Zhaowen, Z., \& Feixue, W. (1998). Mixer-free all digital quadrature demodulation. Proceedings of the Fourth International Conference of the IEEE CSP, 2, 1704-1707.

Grosan, C., \& Abraham, A. (2008). A new. approach for solving nonlinear equations systems. IEEE Transactions on Systems, Man, and Cybernetics-Part A: Systems and Humans, 38(3), 698714.

Ghiglia, D. C., \& Pritt, M. D. (1998). Two-dimensional phase unwrapping theory, algorithms, and software. New York: John Willy \& Sons, Inc.

Garrett, W. E. J. R. (1990). Muscle strain injuries: clinical and basic aspects. Med Sci Sports Exerc., 22(4), 436-43.

Gao, L., Parker, K. J., Lerner, R. M., \& Levinson, S. F. (1996). Imaging of the elastic properties of tissue. Ultrasound in Med. \& Biol., 22(8), 952-977.

Greenleaf, J., Fatemi, M., \& Insana, M. (2003). Selected methods for imaging elastic properties of biological tissues. Annu Rev Biomed Eng., 5, 57-78.

Helliwell, P. S., Mumford, D. B., Smeathers, J. E., \& Wright, V. (1992). Work related upper limb disorder: the relationship between pain, cumulative load, disability, and psychological factors. Ann Rheum Dis., 51(12), 1325-1329.

Hoeger, W. W. K., \& Hoeger, S. A. (2008). Lifetime Physical Fitness and Wellness: A Personalized Program. (10th ed.). United States: Cengage Learning.

Hein, I. A., \& O’Brien, W. D. (1993). Current time-domain methods for assessing tissue motion by analysis from reflected ultrasound echoes. IEEE Trans. Ultrason.,Ferroelect., Freq. Contr., 40(2), 84-102. 
Huang, S., Lerner, R. M., Parker, K. J. (1990). On estimating the amplitude of harmonic vibration from the Doppler spectrum of reflected signal. J. Acoust. Soc. Am., 88(6), 2702-2712.

Hasegawa, H., \& Kanai, H. (2008). Reduction of influence of variation in center frequencies of RF echoes on estimation of artery-wall strain. IEEE Trans Ultrason Ferroelectr Freq Control., 55(9), 1921-34.

Huffel, S. V., \& Vandewalle, J. (1991). The total least squares problem: computational aspects and analysis. Philadelphia: SIAM.

Hodges, P. W., Pengel, L. H. M., Herbert, R. D., \& Gandevia, S. C. (2003). Measurement of muscle contraction with ultrasound imaging. Muscle and Nerve, 27, 682-692.

Hedrick, W. R., Hykes, D. L., \& Starchman, D. E. (2005). Ultrasound physics and instrumentation. (4th ed.). Missouri (USA): Elesvier Mosby.

Hoskins, P., Thrush, A., Martin, K., \& Whittingam, T. (2003). Diagnostic ultrasound: Physics and equipment. London (England): Greenwich Medical Media Limited.

Jin, Q. (2008). A convergence analysis of the iteratively regularized Gauss-Newton method under the Lipschitz condition, 24(4), 1-16.

Järvinen, T. A., Järvinen, T. L., Kääriäinen., M, Aärimaa, V., Vaittinen, S., Kalimo, H., \& Järvinen, M. (2007). Muscle injuries: optimising recovery. Best Pract Res Clin Rheumatol. 21(2), 317-31.

Jones, D. A., \& Round, J. M. (1990). Skeletal muscle in health and disease. United States (NY): Manchester University Press ND.

, J., Tolosa, E. (2007). Parkinson's disease and movement disorders. United States: Lippincott Williams \& Wilkins. 
Kolár̆, R., \& Jiří, J. (2005). Tissue models and speckle reduction in medical ultrasound images. Image analysis, 3540, 1017-1026.

Kremkau, F. W. (2006). Diagnostic ultrasound: Principles and instruments. (7th ed.). Missouri (USA): Saunders Elesvier.

Kremkau, F. W. (2006). Diagnostic ultrasound: Principles and instruments. (7th ed.). Missouri (USA): Saunders Elesvier.

Lawrence (2008). Henderson's dictionary of biology. (14th ed.). London.

Larsson, M., Kremer, F., Claus, P., Brodin, L. A., \& D'hooge, J. (2009). Ultrasound-based 2D strain estimation of the carotid artery: an in-silico feasibility study.

Larson-Meyer, D. E. (2007). Vegetarian sports nutrition. United States: Human Kinetics.

Lionheart, W. R. B. (2011). Reconstruction algorithms for permittivity and conductivity imaging. Proceedings of the 2nd World Congress on Industrial Process, 4-11.

Loupas, T., Powers, J.T., \& Gill, R.W. (1995). An axial velocity estimator for ultrasound blood flow imaging, based on a full evaluation of the Doppler equation by means of a two-dimensional autocorrelation approach. IEEE Trans. Ultrason.,Ferroelect., Freq. Contr., 42(4),672-688.

Mantke, R., Peitz, U. (2007). Surgical ultrasound: An interdisciplinary approach for surgeons, internists, and ultrasound technicians. New York: Georg Thieme Verlag.

Marieb, E. N. (1995). Human anatomy and physiology. (3rd ed.). Redwood City (Calif): Benjamin. 
Molthen, R. C., Narayanan, V. M., Shankar, P. M., Reid, J. M., Genis, V., Forsberg, F., et al. (1998). Using phase information in ultrasonic backscatter for in vivo liver analysis. Ultrasound in Med. \& Biol., 24(1), 79-91.

Matthews, G. G. (2003). Cellular physiology of nerve and muscle. (4th ed.). United Kingdom: Wiley-Blackwell.

Mayo, P. H., \& Doelken, P. (2009). An Introduction to Pleural Ultrasonography for the Pulmonary and Critical Care Physician. Retrieved January 12, 2010, from American Collage of Chest Physicians Web site: http://www.chestnet.org/accp/pccsu/introduction-pleuralultrasonography-pulmonary-and-critical-care-physician?page $=0,3$.

Neves, L. P., Jiang, J., Hall, T. J., Carneiro, A. A. O. (2007). Acoustic elastography under dynamic compression using one-dimensional track motion. Proceedings of the 29th Annual International Conference of the IEEE EMBS.

Narayanan, V. M., Shankar, P. M., \& Reid, J. M. (1994). Non-Rayleigh Statistics of Ultrasonic Backscattered Signals. IEEE Trans. Ultrason.,Ferroelect., Freq. Contr., 41(6), 845-852.

Ophir, J., Cespedes, I., Ponnekanti, H., Yazdi, Y., \& Li, X. (1991). Elastography: a quantitative method for imaging the elasticity of biological tissues. Ultrasonic Imaging, 111-134.

Ophir, J., Alam, S. K., Garra, B., Kallel, F., Konofagou, E., Krouskop, T., et al. (1997). Elastography: ultrasonic estimation and imaging of the elastic properties of tissues. Proc InstnMech Engrs, 213, 203-233.

Prince, J. L., \& Links, J.M. (2006). Medical imaging: Signals and systems. Upper Saddle River (NJ): Pearson Prentice Hall.

Proakis, G. J., \& Manolakis, D. K. (2007). Digital Signal Processing. (4th ed.). New Jersey: Pearson Prentice Hall. 
Plowman, S., \& Smith, D. L. (2007). Exercise physiology for health, fitness, and performance. (2nd ed.) Philadelphia: Lippincott Williams \& Wilkins.

Peachey, L. D., Adrian, R. H., \& Geiger, S. R. (1983). Skeletal muscle, Bethesda (Md): American Physiological Society.

Pinton, G. F., Dahl, J. J., Trahey, G.E. (2006). Rapid tracking of small displacements with ultrasound. IEEE Trans. Ultrason.,Ferroelect., Freq. Contr., 53(6),1103-1117.

Parker, K. J., Gao, L., Alam, S. K., Rubens, D., \& Lerner, R. M. (1996). Sonoelasticity Imaging: Theory and Applications. IEEE Ultrasonic Symposium. 45, 623-28.

Peng, Q., \& Zhang, L. (2006). Ultrasound evaluation of mechanical properties of individual muscles-tendons during active contraction. Proceedings of the 27th Annual International Conference of the IEEE EMBS. 7436-7439.

Physics essays: Cardiac ultrasound machine: Transducers used in the cardiac ultrasound machine. Retrieved May 16, 2009, from UKessays.com Web site: http://www.ukessays.com/essays/physics/cardiac-ultrasound-machine.php.

Qi, Z., Guang, Y., \& Peiwen, Q. (2009). Ultrasonic signals processing base on parameters estimation. Russian Journal of Nondestructive Testing, 45(1), 61-66.

Silver, J. L. (2009). Development of ultrasound based techniques for measuring skeletal muscle motion. Canada (Ottawa): Carleton University.

Souchon, R.(2007). Ultrasonic elastography. Physics for Medical Imaging Applications, 240 (3), 197-209. 
Shamdasani, V., \& Kim, Y. (2004). Two-dimensional autocorrelation method for ultrasoundbased strain estimation. Proceedings of the 26th Annual International Conference of the IEEE EMBS, 1, 1380-1383.

Shankar, P. M. (2000). A general statistical model for ultrasonic backscattering from tissues. IEEE Trans. Ultrason.,Ferroelect., Freq. Contr., 47(3), 727-736.

Silverthorn, D. U. (1998). Human physiology: an integrated approach. New Jersey: Prentice Hall.

Sommerich, C. M., McGlothlin, J. D., \& Marras, W. S. (1993). Occupational risk factors associated with soft tissue disorders of the shoulder: a review of recent investigations in the literature. Erganomics, 36(6), 697-717.

Solly, S. (2007). The classic: clinical lectures on Scrivener's palsy, or the paralysis of writers. Clinical Orthopaedics \& Related Research, 458, 4-9.

Typer, S. (1994). Repetitive strain injury. Journal of Psychosomatic Research, 38(6), 493498. 1994.

Torp, H., Kristoffersen, K., \& Angelsen, B. A. J. (1994). Autocorrelation techniques in color flow imaging: signal model and statistical properties of the autocorrelation estimates. IEEE Trans. Ultrason.,Ferroelect., Freq. Contr., 41(5), 604-612.

Taylor, L. S., Porter, B. C., Rubens, D. J., \& Parker, K.J. (1999). Three-dimensional sonoelastography: principles and practices. Phys. Med. Biol. 45, 1477-1494.

Tatar, F., Mollinger, J. R., Turmezei, P., Bossche A. (2003). Cross-correlation method applied to an ultrasound system for measuring position and orientation of laparoscopic. Proceedings of the XVII IMEKO World Congress. 
Tarantola, A. (2005). Inverse problem theory and methods for model parameter estimation. Philadelphia: SIAM.

Tsui, P., \& Chang, C. (2007). Imaging local scatterer concentrations by the Nakagami statistical model SCATTERER. Ultrasound in Med. \& Biol., 33(4), 608-619.

U.S. National Cancer Institute (2005). An introduction to human anatomy and physiology. Retrieved January 24, 2010, from SweetHaven Publishing Services Web site: http://www.freeed.net/sweethaven/science/biology/anatomyphysiol/human01.asp

Varghese, T. (2009). Quasi-static ultrasound elastography. Ultrasound Clinics, 4(3), 323-338.

Walker, B. (2007). The Anatomy of Sports Injuries. California: North Atlantic Books.

Ylinen, J. J. J., Nurmenniemi, J., Hill, S. (2008). Stretching therapy: for sport and manual therapies. London (UK): Elsevier Health Sciences.

Yu, A. C. H., Steinman, A. H., \& Cobbold, R. S. C. (2006). Transit-time broadening in pulsed Doppler ultrasound: A generalized amplitude modulation model. IEEE Transaction on Ultrasonics, Ferroelectrics and Frequency Control. 53(3), 531-541. 